\title{
Trimethylation Enhancement using Diazomethane (TrEnDi): A Novel Technique to Enhance the Sensitivity of MS-Based Analyses of Biological Molecules
}

\author{
By \\ Karl Vladimir Wasslen \\ A thesis submitted to the Department of Chemistry \\ in partial fulfillment of the requirements for the degree of \\ Master of Science
}

To

\author{
Carleton University \\ Department of Chemistry \\ Ottawa, Canada
}

January 2014

(C) Copyright 2014, Karl Vladimir Wasslen 


\section{Abstract:}

Defining cellular processes relies heavily on elucidating the temporal dynamics of both lipids and proteins. Different mass spectrometry (MS)-based quantitative strategies have emerged to map protein and lipid dynamics over the course of stimuli. We report the development of a novel MS-based quantitative proteomics and lipidomics strategy with unique analytical characteristics. By reacting with diazomethane, analytes are modified to contain fixed, permanent positive charges resulting in improved ionization characteristics and predictable dissociation pathways. Optimization and determination of reactive functional groups enabled a priori prediction of $\mathrm{MS}^{2}$ fragmentation patterns for both modified peptides and lipids. The strategy was tested on digested BSA and successfully quantified a peptide not observable prior to modification. Our chemistry eliminates the need for protonation during ionization, reduces ion suppression, and permits predictable MRM-based or precursor ionbased quantitation with improved sensitivity. 


\section{Acknowledgments}

I would like to extend my sincere gratitude and appreciation to my supervisor and friend, Jeff Smith, for his insight and guidance throughout my university years and for the multiple opportunities to present and network at conferences for which I am grateful. If you ever need a lab manager... we can discuss the terms! I thank Jeff Manthorpe for his guidance and efforts to help me succeed and for always helping me unconditionally to better my research.

I also thank my parents Larry and Ninfa Wasslen for providing me all the essentials in life and for their continuous support. Their nourishing guidance has provided me with life experiences that have shaped who I am today and for that I am forever grateful. Hasta la victoria siempre! To Nick, comrade, brother and best mate, for always being there for me, especially when I need you the most! Special thanks to my sister Yamile and her husband Jamie, who babysat countless times when I was desperate. To past and present Smith Lab members... you guys rock! I thank my best friends Dan and Mike for always being there for me, the friends I have made while playing for the Ravens and Henri and Renee for their unconditional friendship.

Lastly and foremost, I thank the love of life... my wife Anna. She has been my rock in times of difficulty and has always been patient, encouraging, and ever enduring. She has truly been the pinnacle to my success since I've met her. Ich liebe dich MEHR! I thank my two lovely children, William and Vanessa for their love and laughter, which have always made my day brighter and full of joy (even when some days it really hasn't). It is to these three that I dedicate this work, my graduate degree and all my future success. 


\section{Table of Contents}

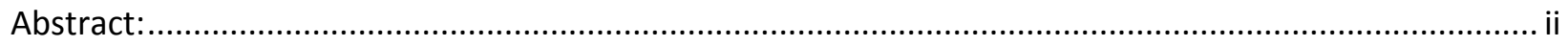

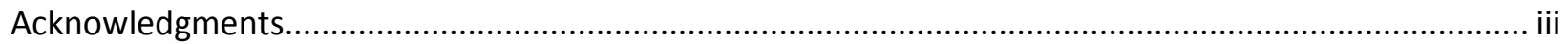

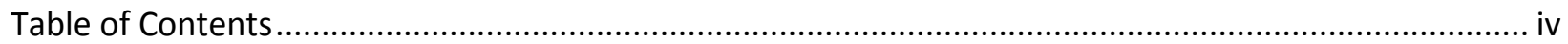

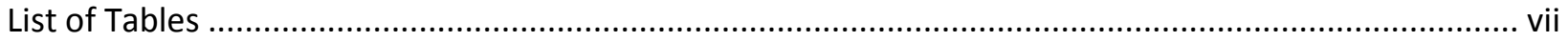

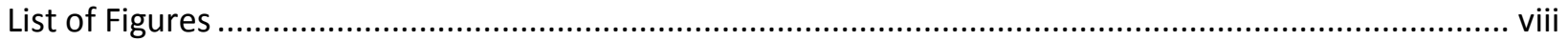

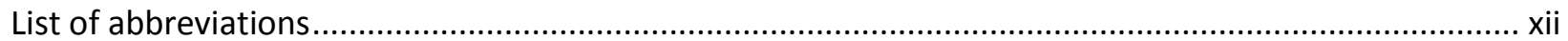

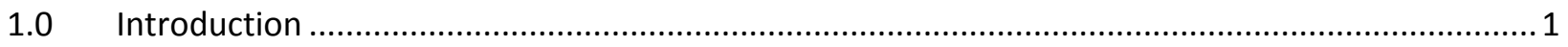

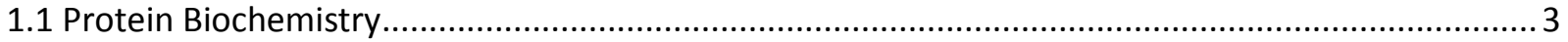

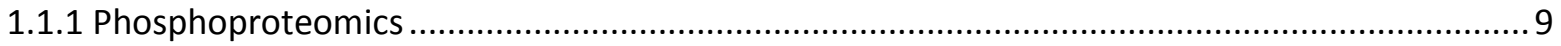

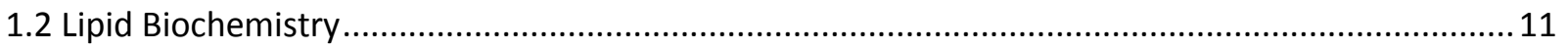

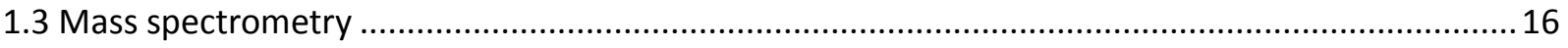

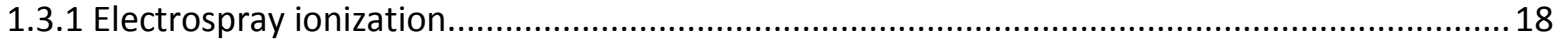

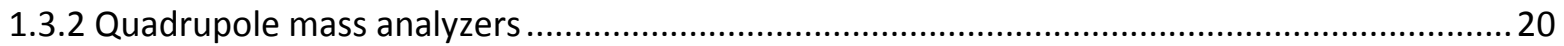

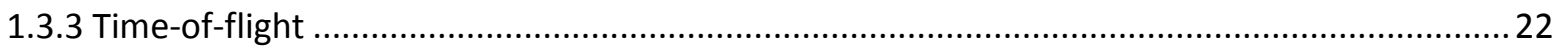

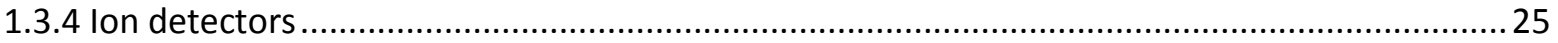

1.3.5 Hybrid quadrupole-time-of-flight mass spectrometer ..........................................................26

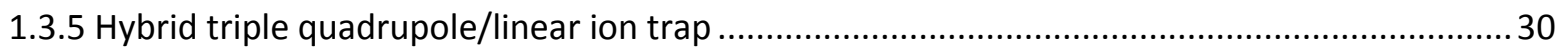

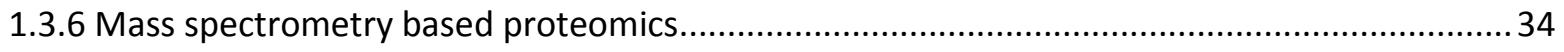

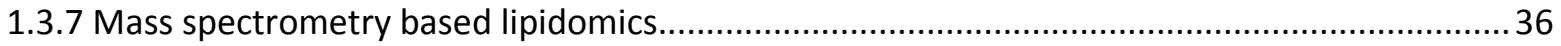

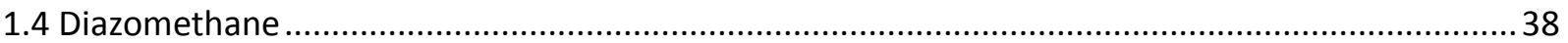

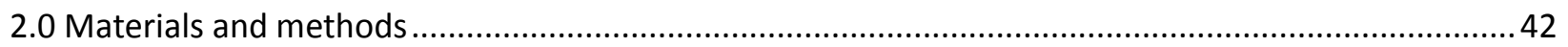

$2.1 \mathrm{~N}$-methyl- $\mathrm{N}$-nitroso- $p$-toluenesulfonamide (Diazald) production ................................................43

2.2 Diazomethane production ................................................................................................... 44

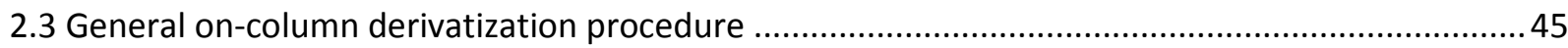

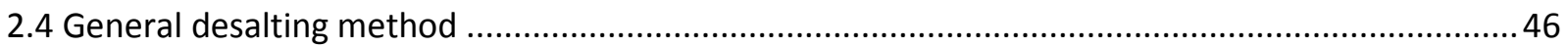

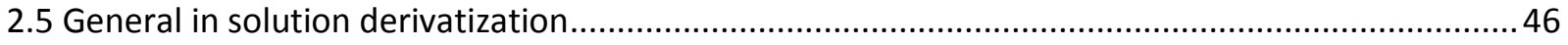

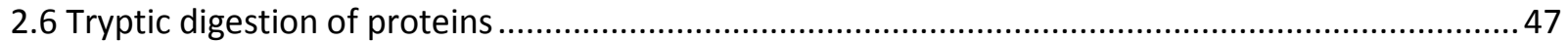

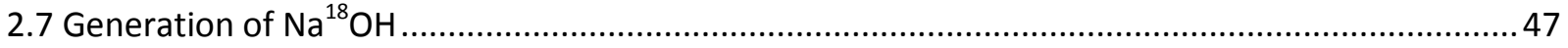




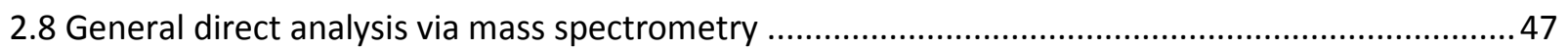

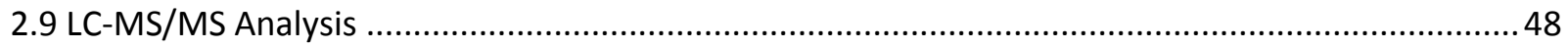

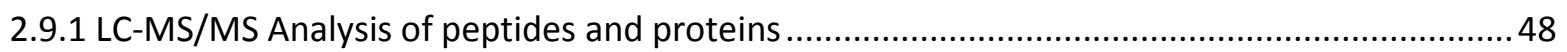

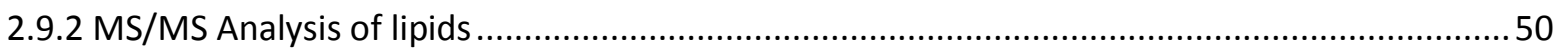

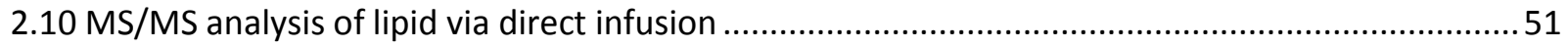

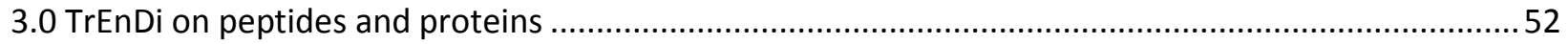

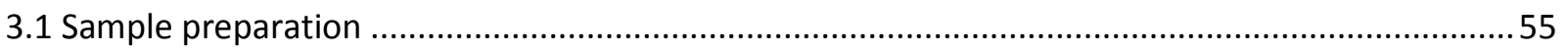

$3.2 \mathrm{~N}$-methyl- $\mathrm{N}$-nitroso- $p$-toluenesulfonamide (Diazald) production ................................................55

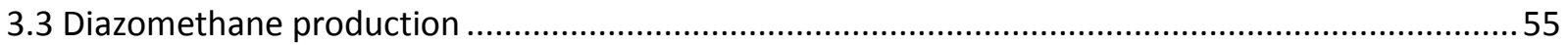

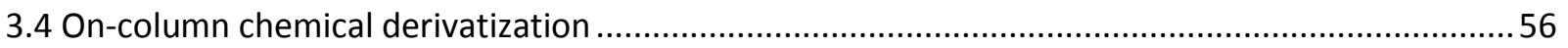

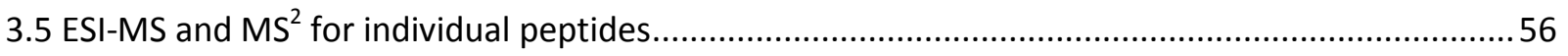

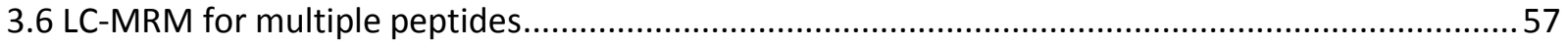

3.7 BSA protein identification and LC-MRM for modified BSA ..........................................................57

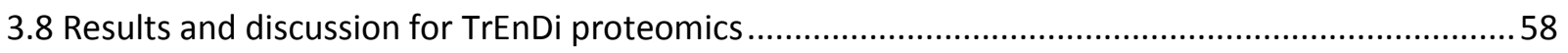

3.8.1 Tandem Mass Spectrometry of Methylated Peptides ........................................................68

3.8.2 Multiple peptides tested simultaneously ........................................................................... 72

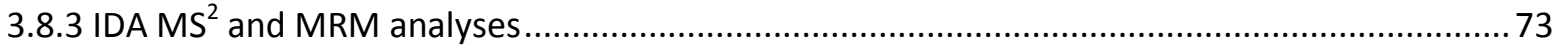

3.8.4 Diazomethane efficiency on a protein digest ................................................................. 75

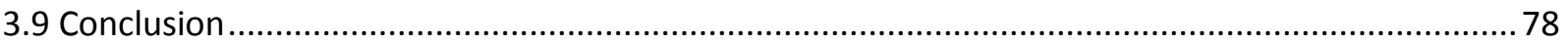

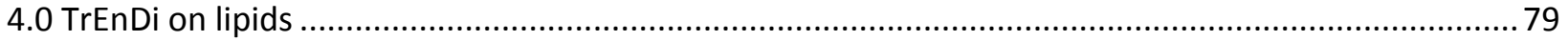

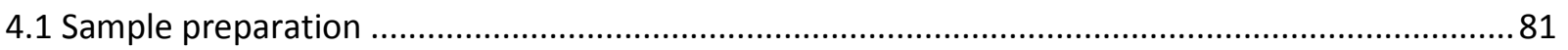

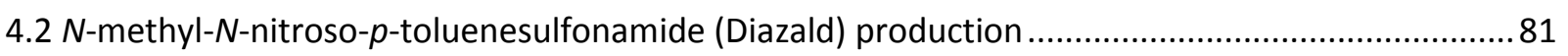

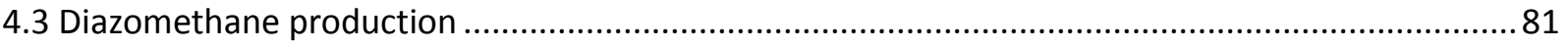

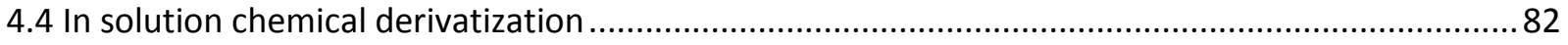

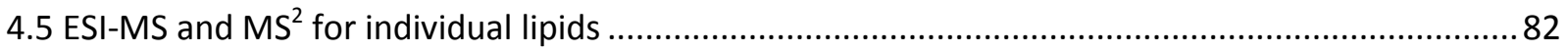

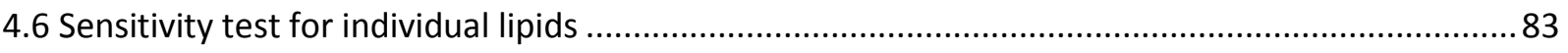

4.7 Shotgun lipidomics Precursor ion scanning for multiple lipids .................................................... 83

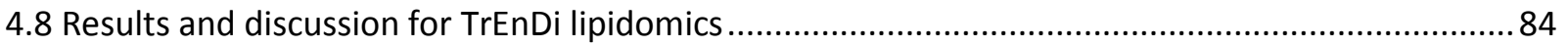

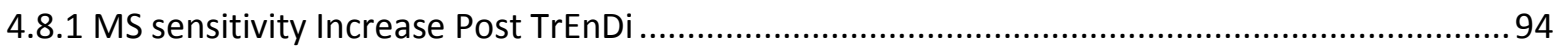

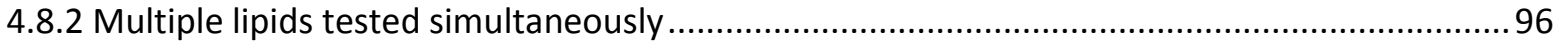

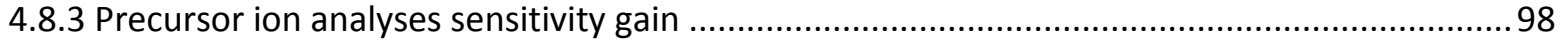

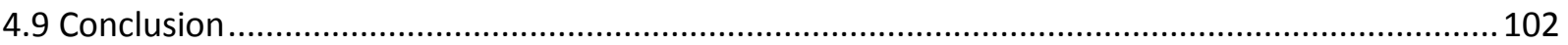




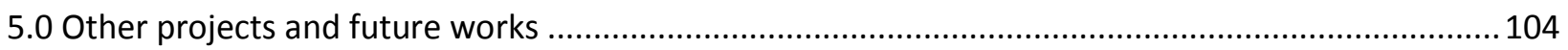

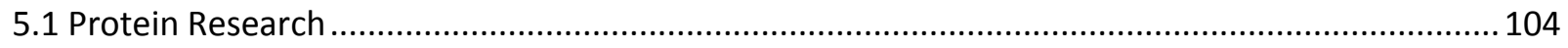

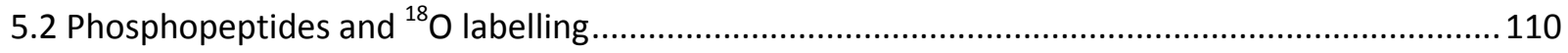

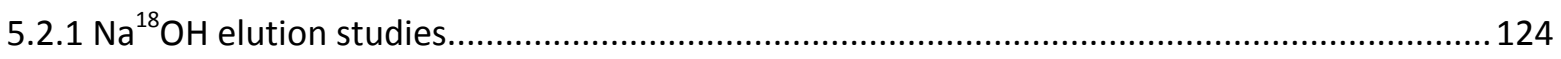

5.3 On-column derivatization and elution with triethylammonium acetate ....................................127

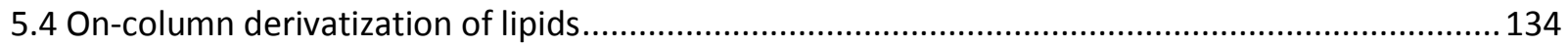

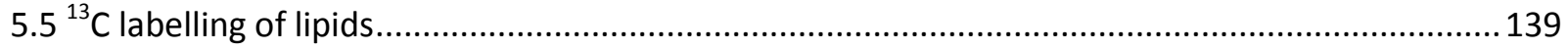

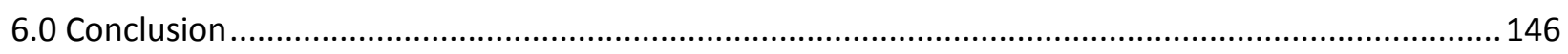

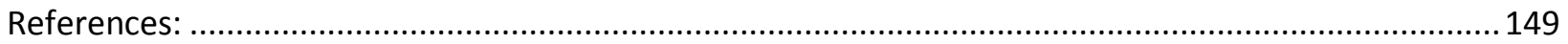




\section{List of Tables}

Table 3.1: Summary of results for diazomethane-modified synthetic peptides

Table 3.2: Summary of functional groups tested with TrEnDi and the resulting structure post-TrEnDi modification .66

Table 3.3: Summary of MRM transitions for TrEnDi modified peptides .73

Table 4.1: Summary of results for diazomethane-modified synthetic lipids .93

Table 4.2: Summary of interesting transitions for unmodified and TrEnDi-modified lipids....................101

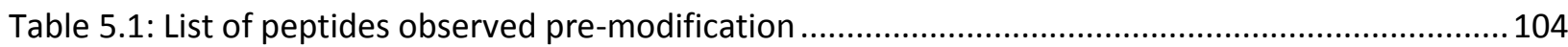

Table 5.2: List of peptides observed post-TrEnDi modification .......................................................... 105

Table 5.3: Predicted $a, b$ and $y$ ions versus observed ions highlighted in yellow in the $\mathrm{MS}^{2}$ analysis of ALELFR

Table 5.4: Predicted $a, b$ and $y$ ions versus observed ions highlighted in yellow in the $\mathrm{MS}^{2}$ analysis of LFTGHPETLEK 108

Table 5.5: Predicted $a, b$ and $y$ ions versus observed ions highlighted in yellow in the $\mathrm{MS}^{2}$ analysis of VEADIAGHGQEVLIR. 109

Table 5.6: Summary of results for diazomethane-modified synthetic peptides followed by elution with $\mathrm{Et}_{3} \mathrm{~N}^{+} \mathrm{H}^{-} \mathrm{OAC}$ 


\section{List of Figures}

Figure 1.1: The central dogma of molecular biology ....................................................................... 3

Figure 1.2: The basic configuration of an amino acid under physiological conditions ................................ 4

Figure 1.3: The list of 20 amino acids grouped based on their hydrophobicity. Adapted from Protein-

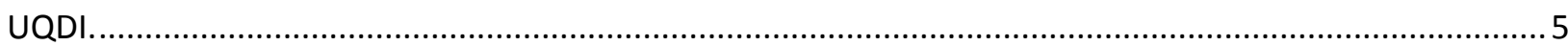

Figure 1.4: Condensation reaction of a carboxylic acid with a primary amine to produce the peptide bond

Figure 1.5: The four structural levels of proteins. A) the primary structure; B) common secondary structures of proteins (alpha helix and beta sheets); C) a tertiary structure of a protein (PI3); D) the quaternary structure of hemoglobin. Reproduced from Wikimedia Commons. ....................................... 8

Figure 1.6: Reversible reaction of phosphorylation on $\mathrm{R}$. $\mathrm{R}$ implies a serine, threonine or tyrosine residue.

Figure 1.7: Representative structures of the 8 lipid classes. Lipid classes of interest are boxed. Adapted

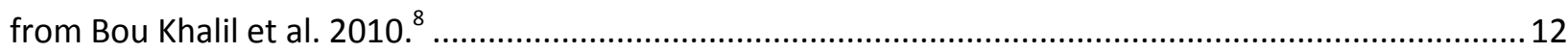

Figure 1.8: The general structure of a glycerophospholipid ...............................................................13

Figure 1.9: The 8 glycerophospholipid subclasses. Those highlighted are of particular relevance to this thesis. Adapted from Bou Khalil et al 2010. ${ }^{8}$

Figure 1.10: Representative structures of sphingolipids. Highlighted is the phosphosphingolipid commonly known as sphingomyelin. Adapted from Bou Khalil et al 2010. ${ }^{8}$...........................................15

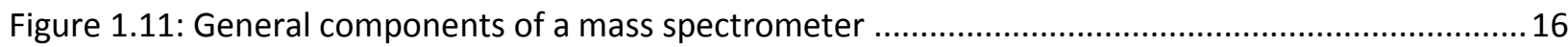
Figure 1.12: General overview of electrospray ionization. The reduction electrode would have an orifice

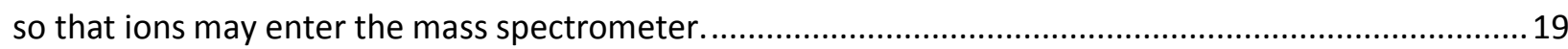

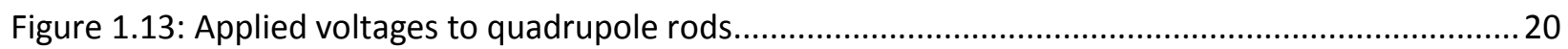

Figure 1.14: High-bandpass filtering in the xz plane and low-bandpass filtering in the yz plane. Adapted

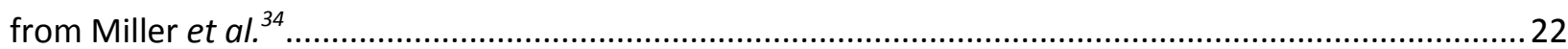

Figure 1.15: General schematic of time-of-flight mass spectrometry.................................................23

Figure 1.16: Ion mirror correcting the same $\mathrm{m} / \mathrm{z}$ ion with different velocities ......................................24

Figure 1.17: Microchannel plate detector. Ion impingement releases a cascade of electrons towards the

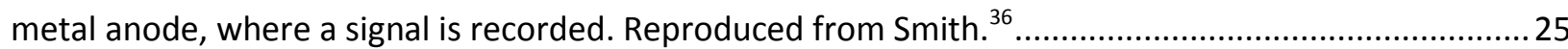

Figure 1.18: General schematic of a QqTOF mass spectrometer. Adapted from Smith. ${ }^{36}$.........................26

Figure 1.19: Sample ions produced by ESI are being introduced to the front end of the QqTOF. "CP" refers to curtain plate; "CG" refers to curtain gas; "OR" refers to orifice; "SE" refers to supersonic

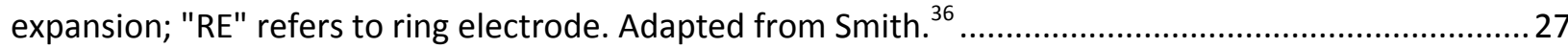

Figure 1.20: The detector of the QqTOF mass spectrometer. Adapted from Smith. ${ }^{36}$.............................29 Figure 1.21: General schematic diagram of a triple quadrupole mass spectrometer. Reproduced from de

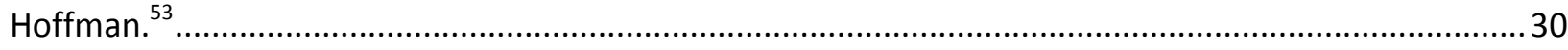

Figure 1.22: Example of a chromatogram obtained from MRM analysis ..............................................32

Figure 1.23: $\mathrm{MS}$ and $\mathrm{MS}^{2}$ experiments. Between the mass analyzers is a collision cell containing an inert

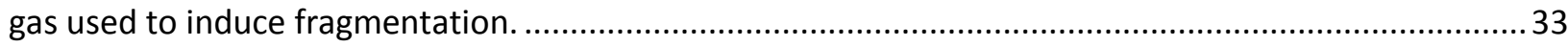


Figure 1.24: Formation of $a-,, b-, c-$ and $x-, y-$ and $z-$ ions from fragmentation of tetramethylated hexaglycine. ${ }^{62}$

Figure 1.25: A) The precursor fragment for PC and SM; B) the neutral loss fragment for PE; C) the neutral

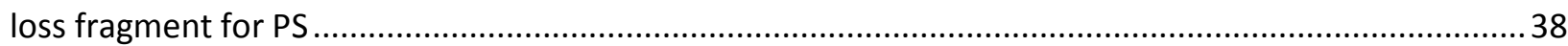

Figure 1.26: Two step reaction to synthesize diazomethane .............................................................39

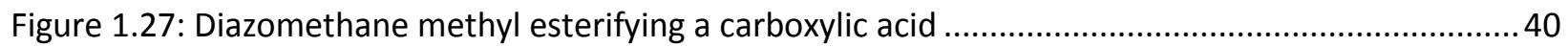

Figure 2.1: A gradient used for peptide elution from HPLC. $A=\mathrm{H}_{2} \mathrm{O}$ and $0.1 \%$ formic acid. $B=A C N$ and $0.1 \%$ formic acid.

Figure 2.2: Gradient used for lipid elution. $A=30 \%$ methanol $/ 70 \%$ water $/ 10 \mathrm{mM}$ ammonium acetate;

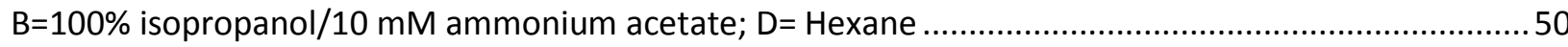

Figure 3.1: Methylation of peptide N-terminus with diazomethane..................................................59

Figure 3.2: a) FLEEVK treated with diazomethane and eluted with ammonium bicarbonate revealing nine added methyl groups ( $\mathrm{m} / \mathrm{z} 445.78$ ). b) Fragmentation of this peptide revealed $y$-ions and a predominant $a_{2}$ fragment. 60

Figure 3.3: Fully modified FLEEVK via treatment with diazomethane.

Figure 3.4: a) FLEEVK treated with diazomethane and eluted with sodium hydroxide revealing six added methyl groups ( $m / z$ 424.76). b) Fragmentation of this peptide revealed $y$-ions, $y$-ion with the loss of water, immonium ions and a predominant $\mathrm{a}_{2}$ fragment.

Figure 3.5: Hexamethylated FLEEVK via reaction with diazomethane followed by complete hydrolysis of the methyl esters using $\mathrm{NaOH}$

Figure 3.6: MS analysis of FLEESK ( $\mathrm{m} / \mathrm{z} 418.8$ ) and FLEETK ( $\mathrm{m} / \mathrm{z} 425.8)$ show that primary and secondary alcohol groups do not easily methylate (less than 10\%) within the time frame of our experiment..........65 Figure 3.7: a) $\mathrm{MS}^{2}$ of TrEnDi-modified FLEEVR revealing that the guanidinium side chain of arginine does not get methylated. b) $\mathrm{MS}^{2}$ of TrEnDi-modified FLEENK revealing that the amide side chain of asparagine does not get methylated. c) MS ${ }^{2}$ of TrEnDi-modified FLEEYK revealing that the phenol side chain of tyrosine does get methylated. All ions fragment to form a y-ion series and a predominant $\mathrm{a}_{2}$ fragment.70 Figure 3.8: a) MS of TrEnDi-modified FLEEHK (m/z 305.52). b) This peptide is triply charged as revealed by the molecular ion isotopic spacing of $\Delta 0.33 \mathrm{Th}$. c) $\mathrm{MS}^{2}$ of TrEnDi-modified FLEEHK revealing $\mathrm{y}$-ion series, a y-ion series with a water loss, the immonium ion of histidine and the characteristic $a_{2}$ fragment ion.

Figure 3.9: a) MS spectrum of a mixture of TrEnDi-modified FLEEVR, FLEEVK, FLEENK and FLEEYK. b) MRM chromatogram of these same four peptides spiked into a concentrated complex mixture of digested protmix (see Section 3.6) and successfully identified by two distinct parent ion to fragment ion transitions including the $a_{2}$ fragment ion in every case.

Figure 3.10: MRM chromatogram of BSA peptide AEFVEVTK with an inset of the BSA amino acid sequence. The regions identified by Mascot on data acquired using an IDA experiment are shown in red and the peptide AEFVEVTK that was successfully identified using TrEnDi is underlined in bold font. ......77 Figure 4.1: Methylation of a phosphoethanolamine lipid head group with diazomethane..... .86 Figure 4.2: a) PE unmodified revealing protonated and sodiated parent ions. b) Fragmentation of the unmodified PE indicates divided signal along the various fragmentation channels.

Figure 4.3: a) PE treated with diazomethane reveals four methyl groups ( $\mathrm{m} / \mathrm{z} 774.6)$. b) Fragmentation of this lipid revealed a single fragmentation channel $(\mathrm{m} / \mathrm{z} 198.1)$ 88 
Figure 4.4: Fully modified PE (16:0-18:1) via treatment with diazomethane and $\mathrm{HBF}_{4}$ 89 Figure 4.5: a) PS unmodified revealing three parent ions (protonated, sodiated and doubly sodiated). b) Fragmentation of the unmodified PS at $\mathrm{m} / \mathrm{z} 788.5$ indicates divided signal along the various fragmentation channels.

Figure 4.6: a) PS treated with diazomethane reveals five methyl groups ( $\mathrm{m} / \mathrm{z} 858.6)$. b) $\mathrm{MS}^{2}$ reveals two dominant fragmentation channels (m/z 256.1 and 144.1 respectively) ..............................................91

Figure 4.7: Pentamethylated PS(18:0/18:2(9Z,12Z)) via reaction with diazomethane and $\mathrm{HBF}_{4} \ldots \ldots \ldots \ldots . . . .91$

Figure 4.8: a) PC treated with diazomethane reveals addition of one methyl group ( $\mathrm{m} / \mathrm{z} 746)$. b)

Fragmentation of this lipid revealed one dominant fragmentation channel similar to the expected 184 Da head group with the additional methyl group ( $\mathrm{m} / \mathrm{z}$ 198.1)

Figure 4.9: a) SM treated with diazomethane reveals addition of one methyl group ( $\mathrm{m} / \mathrm{z} 717.6)$. b) Fragmentation of this lipid revealed one dominant fragmentation channel similar to the expected 184 Da head group with the additional methyl group ( $\mathrm{m} / \mathrm{z}$ 198.1)

Figure 4.10: a) Equimolar amounts of modified and unmodified PE reveals an increase in sensitivity by nearly 60 percent. b) Equimolar amounts of modified and unmodified PS shows nearly $100 \%$ increase in sensitivity. c) Equimolar amounts of modified and unmodified PC show no significant increase in sensitivity. d) Equimolar amounts of modified and unmodified SM show an increase in sensitivity. .......95 Figure 4.11: MS spectrum of mixture of TrEnDi-modified SM, PC, PE and PS......................................96 Figure 4.12: MS reveals the sensitivity increase gained from TrEnDi modified lipids over an equimolar

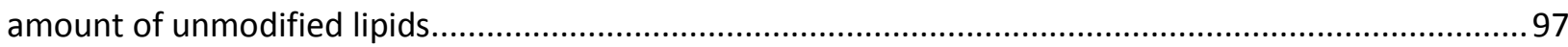

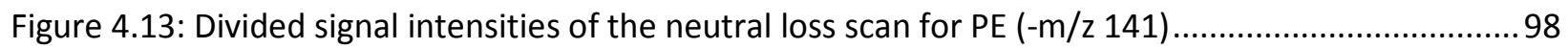

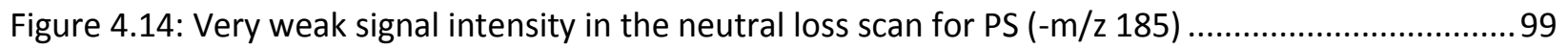
Figure 4.15: Relatively strong signal intensities for the precursor ion scan ( $\mathrm{m} / \mathrm{z} 184)$ for PC $(\mathrm{m} / \mathrm{z} 732.7)$ and SM (m/z 703.7)....

Figure 4.16: Significantly stronger signal intensities for the precursor ion scan ( $\mathrm{m} / \mathrm{z} 198)$ for TrEnDimodified SM ( $\mathrm{m} / \mathrm{z} 717.7), \mathrm{PC}(\mathrm{m} / \mathrm{z} 746.7)$ and PE ( $\mathrm{m} / \mathrm{z} 774.7)$ 100 Figure 4.17: Significantly stronger signal intensity in the precursor ion scan (m/z 256) for TrEnDimodified PS ( $\mathrm{m} / \mathrm{z}$ 858.7). 100

Figure 4.18: The strongest signal intensity for the precursor ion scan $(\mathrm{m} / \mathrm{z} 144)$ with CE of $60 \mathrm{eV}$ for

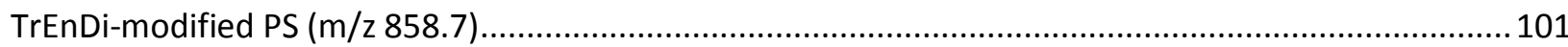

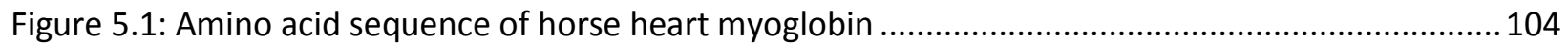

Figure 5.2: MS analysis of myoglobin confirms three peptides fully modified ....................................106

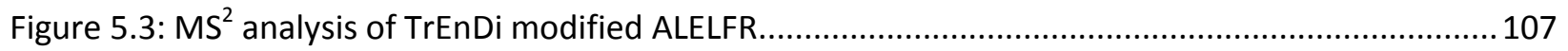

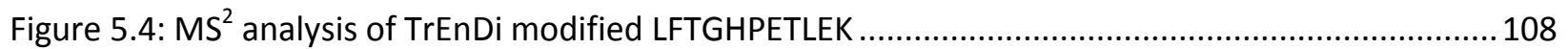

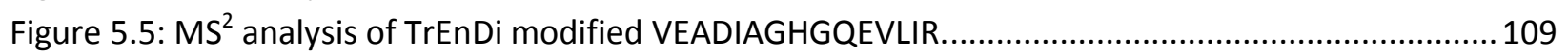

Figure 5.6: MS results of FLEEpSK prior to TrEnDi modification............................................................... 111

Figure 5.7: $\mathrm{MS}^{2}$ results of unmodified FLEEpSK. All y ions represent the mass of the ion with a neutral

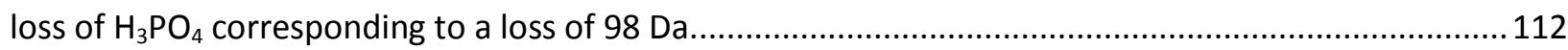

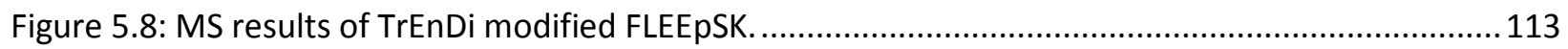

Figure 5.9: $\mathrm{MS}^{2}$ results of modified FLEEpSK at $\mathrm{m} / \mathrm{z}$ 409.7. All $\mathrm{y}$ ions have been dephosphorylated

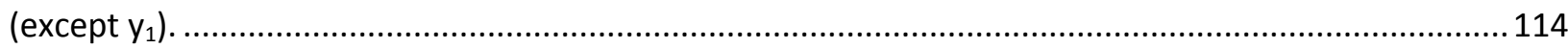

Figure 5.10: Fully modified FLEEpSK with the dephosphorylated serine .............................................115 
Figure 5.11: $\mathrm{MS}^{2}$ analysis of TrEnDi-modified FLEEpSK with an additional ME ( $\left.\mathrm{m} / \mathrm{z} 416.78\right)$....... .116

Figure 5.12: MS analysis of unmodified FLEEPYK

Figure 5.13: $\mathrm{MS}^{2}$ analysis of unmodified FLEEPYK at $\mathrm{m} / \mathrm{z}$ 454.7. All $\mathrm{y}$ ions represent the mass of the ion with a neutral loss of $\mathrm{H}_{3} \mathrm{PO}_{4}$ corresponding to a loss of $98 \mathrm{Da}$.

Figure 5.14: MS analysis of on-column TrEnDi modified FLEEPYK.

Figure 5.15: $\mathrm{MS}^{2}$ analysis of $\mathrm{m} / \mathrm{z}$ 454.7. All y ions are fragment masses of the dephosphorylated parent ion except $\mathrm{y}_{1}$

Figure 5.16: $\mathrm{MS}^{2}$ analysis of $\mathrm{m} / \mathrm{z}$ 463.7. All y ions are fragment masses of the dephosphorylated parent ion except $\mathrm{y}_{1}$

Figure 5.17: $\mathrm{MS}^{2}$ analysis of $\mathrm{m} / \mathrm{z} 503.7$ with the phosphate group still intact.

Figure 5.18: A) MS analysis of TrEnDi modified FLEEpYK eluted with iodized salt. B) Zoom in on the saturation of $\mathrm{m} / \mathrm{z} 531.78$

Figure 5.19: $\mathrm{MS}^{2}$ analysis of $\mathrm{m} / \mathrm{z}$ FLEEpYK with methyl esters and dimethyl phosphate still intact. .......124

Figure 5.20: MS of TrEnDi modified FLEEpSK and elution with $\mathrm{Na}^{18} \mathrm{OH}$.

Figure 5.21: $\mathrm{MS}^{2}$ comparison between TrEnDi modified FLEEpSK and elution with $\mathrm{Na}{ }^{16} \mathrm{OH}$ and $\mathrm{Na}^{18} \mathrm{OH}$

Figure 5.22: Fully modified FLEEpSK via treatment with diazomethane and $\mathrm{Na}{ }^{18} \mathrm{OH}$

Figure 5.23: MS analysis of fully modified FLEEVR, FLEEVK, FLEENK and FLEEYK post TrEnDi modification and elution with $\mathrm{Et}_{3} \mathrm{~N}^{+} \mathrm{H}^{-} \mathrm{OAc}$.....

Figure 5.24: FLEEVK subjected to diazomethane treatment and triethylammonium acetate elution yields a fully modified FLEEVK with 9 methylations and 2 fixed positive charges. 130

Figure 5.25: $\mathrm{MS}^{2}$ results of fully modified FLEEVR (m/z 438.7) with methyl esterification....................132

Figure 5.26: $\mathrm{MS}^{2}$ results of fully modified FLEEVK (m/z 445.3) with methyl esterification......................132

Figure 5.27: $\mathrm{MS}^{2}$ results of fully modified FLEENK (m/z 453.2) with methyl esterification.....................133

Figure 5.28: $\mathrm{MS}^{2}$ results of fully modified FLEEYK (m/z 484.8) with methyl esterification. .....................133

Figure 5.29: PC (16:0, 18:1) modified via on-column TrEnDi............................................................. 135

Figure 5.30: $\mathrm{MS}^{2}$ of fully modified PC $(16: 0,18: 1)$ with one dominant fragmentation channel. .............136

Figure 5.31: PE (16:0, 18:1) modified via on-column TrEnDi ........................................................... 137

Figure 5.32: $\mathrm{MS}^{2}$ of fully modified PE $(16: 0,18: 1)$ with one dominant fragmentation channel. .............138

Figure 5.33: Sensitivity test on equimolar amounts of unmodified vs. TrEnDi modified PE (16:0, 18:1).139

Figure 5.34: MS analysis of PC $(16: 0,18: 1)$ fully modified with ${ }^{13} \mathrm{C}$ diazomethane ................................ 140

Figure 5.35: MS analysis of PE $(16: 0,18: 1)$ fully modified with ${ }^{13} \mathrm{C}$ diazomethane ................................141 Figure 5.36: MS analysis of both PC and PE $(16: 0,18: 1)$ post TrEnDi modification with ${ }^{13} \mathrm{C}$ diazomethane. The three Da mass shift between both species allows for easy identification.......................................142 Figure 5.37: $\mathrm{MS}^{2}$ analysis of $\mathrm{m} / \mathrm{z} 775.6$ fully modified PC $(16: 0,18: 1)$ with ${ }^{13} \mathrm{C}$ diazomethane ...............143 Figure 5.38: $\mathrm{MS}^{2}$ analysis of $\mathrm{m} / \mathrm{z} 778.6$ fully modified $\mathrm{PE}(16: 0,18: 1)$ with ${ }^{13} \mathrm{C}$ diazomethane................144 Figure 5.39: A) Precursor ion scan for $m / z$ 199, characteristic fragment of PC $(16: 0,18: 1)$ fully modified with ${ }^{13} \mathrm{C}$ diazomethane; B) MS analysis on the Qtrap of both PC and PE $(16: 0,18: 1)$ fully modified with ${ }^{13} \mathrm{C}$ diazomethane; $\left.C\right)$ Precursor ion scan for $\mathrm{m} / \mathrm{z} 202$, characteristic fragment of PE $(16: 0,18: 1)$ fully modified with ${ }^{13} \mathrm{C}$ diazomethane. .145 


\section{List of abbreviations}

Acetonitrile $\quad A C N$

Ammonium bicarbonate $\quad A B C$

Atmospheric pressure chemical ionization $\quad \mathrm{APCl}$

Bovine serum albumin BSA

Channel electron multiplier CEM

Collision energy CE

Collision induced dissociation CID

Dalton Da

Direct current DC

Dithiothreitol DTT

Electron impact EI

Electrospray ionization ESI

Enhanced mass scanning EMS

Gas chromatography GC

High performance liquid chromatography HPLC

High pressure vessel HPV

Human Genome Project HGP

Information dependent acquisition IDA

Mass spectrometry MS

Mass-to-charge $\mathrm{m} / \mathrm{z}$

Matrix assisted laser desorption ionization MALDI

Microchannel plate MCP

Multiple reaction monitoring MRM

$N$-methyl- $p$-toluenesulfonamide NMPTS

Phosphatidylcholine PC

Phosphatidylethanolamine PE

Phosphatidylserine PS

Post-translational modification PTM

Potassium phosphate buffer KPB

Radio frequency RF

Selected reaction monitoring SRM

Sphingomyelin SM

Strong cation exchange $\quad$ SCX

Tandem mass spectrometry $\quad \mathrm{MS}^{2}$ or MS/MS

Tetrafluoroboric acid $\mathrm{HBF}_{4}$

Thomson Th

Time-of-flight TOF

Trimethylation Enhancement using Diazomethane TrEnDi

Trimethylsilyldiazomethane TMSD 


\subsection{Introduction}

The Human Genome Project (HGP), a massive collaboration between scientists of various fields, (such as biologists, physicists, chemists, computer scientists, mathematicians and engineers) took 13 years and $\$ 3$ billion $^{1}$ to determine the complete sequence of the human genome. ${ }^{2}$ Since the HGP was completed in $2001,{ }^{1}$ scientists have been able to use the data resulting from the HGP to make further advancements in medicine, ${ }^{3}$ and to increase our understanding of human biological systems. According to a study in 2010, since the HGP began in 1988, it has generated nearly 711000 job-years ${ }^{*}$ and that every \$1 of HGP investment has helped to generate $\$ 141$ in the US economy. ${ }^{2}$ The HGP has also greatly influenced other fields of research, including proteomics, the large-scale study of proteins, and lipidomics, the largescale study of lipids. While genomics led to proteomics and lipidomics, advances in analytical instrumentation, including the Nobel Prize-winning work ${ }^{4}$ of John Fenn ${ }^{5}$ and Koichi Tanaka ${ }^{6}$ for their development of soft ionization techniques have enabled large scale proteomic and lipidomic studies to be conducted using mass spectrometry.

Mass spectrometry (MS) has emerged as the leading analytical tool for quantitative proteomic $^{7}$ and lipidomic ${ }^{8}$ analyses. MS has since been used to quantify both lipids ${ }^{9,10}$ and proteins in living systems ${ }^{11}$ as well as fluctuations in how they are modified. ${ }^{12}$ MS has also facilitated the monitoring of protein concentrations as a medical diagnostic tool ${ }^{11,13,14}$ and permitted the discovery of novel biomarkers. ${ }^{15,16,17}$ Despite recent technological advances and the emergence of a great deal of literature on MS-based quantitative proteomics ${ }^{12,18}$ and

\footnotetext{
${ }^{*}$ Job-year is equivalent to one person employed full time for one year
} 
lipidomics, ${ }^{8,19}$ the dynamics and function of many proteins and lipids remains elusive, particularly for analytes of low relative abundance. Much remains to be understood regarding cellular adaptive proteomic and lipidomic responses, highlighting a need for new analytical tools in this area.

This thesis describes the development of a novel technique termed Trimethylation Enhancement using Diazomethane (TrEnDi) and is better appreciated in the context of a foundational understanding of protein and lipid biochemistry, MS instrumentation as well as MS-based proteomics and lipidomics. 


\subsection{Protein Biochemistry}

The central dogma of molecular biology ${ }^{20}$ describes the flow of genetic information from genes to proteins within a biological system. Figure 1.1 depicts the central dogma of molecular biology: the transfer of information from one polymer with a defined sequence to another. ${ }^{20}$ Briefly, DNA is transcribed into mRNA, which is later translated into proteins.

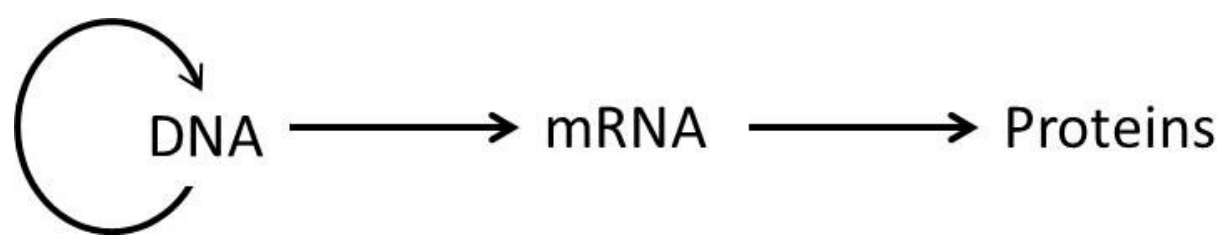

Figure 1.1: The central dogma of molecular biology

Proteins are long polymer chains composed of monomer units called amino acids. Virtually all proteins are composed of 20 'standard' amino acids. ${ }^{21}$ All amino acids consist of a primary amine group $\left(-\mathrm{NH}_{2}\right)$, a carboxylic acid $(-\mathrm{COOH})$ and a side chain (commonly denoted as R) bonded to an $\alpha$ carbon atom as illustrated in Figure 1.2. Proline is the only amino acid that is an exception to this rule as it has a secondary amine group (-NH-). Figure 1.3 illustrates the complete chemical structures of the 20 amino acids. 


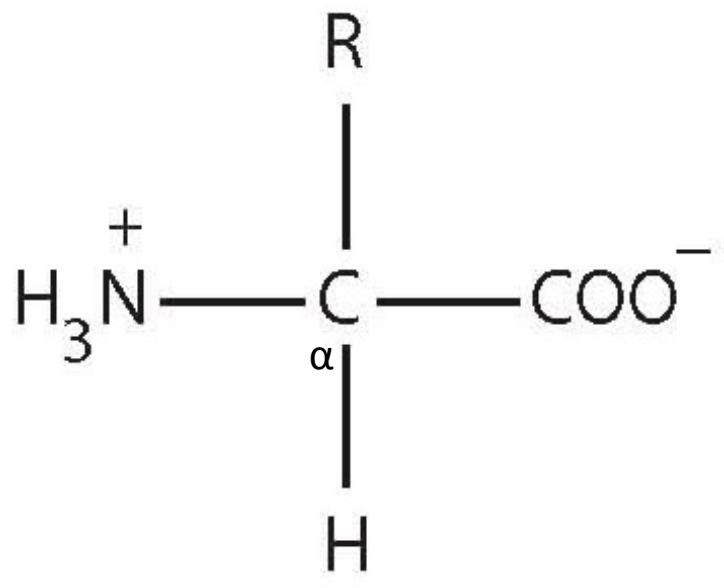

Figure 1.2: The basic configuration of an amino acid under physiological conditions

All 20 amino acids differ from each other because of their functional group, which can be characterized by its hydrophobicity. ${ }^{22}$ There are 8 hydrophobic amino acids, 9 hydrophilic amino acids and 3 that are characterized as special, as they have unique properties. Proline, as previously mentioned, is the only amino acid with a secondary amine group, due to its ring structure. Cysteine is classified as a special amino acid as it has a primary thiol group that may form disulfide bridges with other cysteine residues. Glycine is the only amino acid that lacks a chiral $\alpha$ carbon; as illustrated in Figure 1.2, all other amino acids have 4 distinct groups bound to the $\alpha$ carbon and therefore have two enantiomer forms, D and L. Biological systems are almost exclusively composed of $L$ amino acids. 


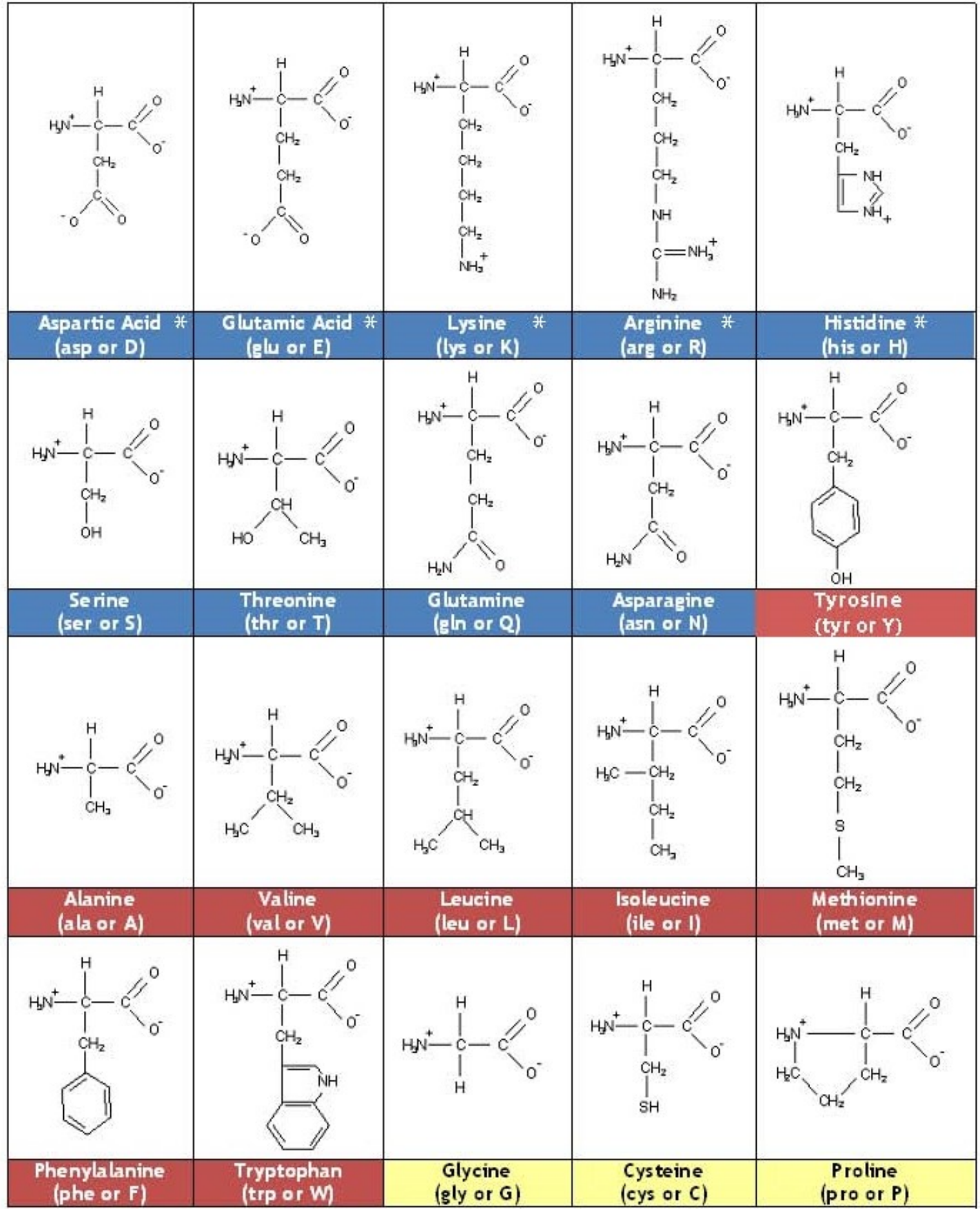

* Charged

Figure 1.3: The list of 20 amino acids grouped based on their hydrophobicity. Adapted from Protein-UQDI. ${ }^{23}$ 
Proteins are composed of amino acids that are bound together through numerous condensation reactions from the amine group of one amino acid to the carboxylic group of another (Figure 1.4). These are known as peptide bonds and form the backbone or primary structure of a protein. The functional groups or side chains of the amino acids give the protein its functionality. Every protein possesses two distinct ends, one containing a basic free amine group known as the $\mathrm{N}$-terminus and one containing an acidic free carboxylic group known as the C-terminus. Under physiological conditions, the basic $\mathrm{N}$-terminus is protonated and the acidic C-terminus is deprotonated. The hydrophobicity of the side chains will determine the overall structure of the protein. Commonly, hydrophilic portions of the protein are directed outwards towards the surrounding environment, while hydrophobic portions of the protein are folded inwards. These properties play a major role in the overall structures of proteins classified as primary, secondary, tertiary and quaternary structures as illustrated in Figure 1.5.

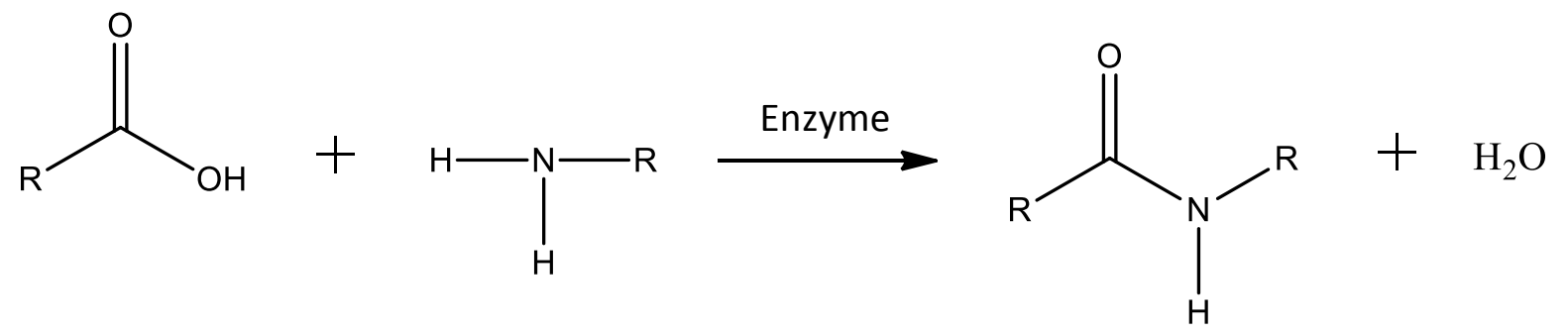

Figure 1.4: Condensation reaction of a carboxylic acid with a primary amine to produce the peptide bond 
The primary structure is the linear sequence of amino acids of a peptide or polypeptide chain. A peptide is defined as a fragment or piece of a protein with varying number of amino acids. The secondary structure is the local spatial arrangement of a polypeptide's backbone atoms without regard to the conformations of its side chains. ${ }^{21}$ These local arrangements consist most commonly of $\alpha$-helices and $\beta$-sheets. $\alpha$-helices are held together via hydrogen bonding between $\mathrm{C}=\mathrm{O}$ groups and $\mathrm{N}-\mathrm{H}$ groups in the backbone of the same polypeptide chain and contain approximately 3.6 residues per turn. $\beta$-sheets are planar pleated structures also held together by hydrogen bonding; however bonding occurs between neighbouring chains rather than within one as in the $\alpha$-helix. Tertiary structures are the total 3 dimensional conformational structures of folded proteins, consisting of all the secondary structural elements with specific locations and include all forms of interactions including those involving the side chains. There is also a quaternary structure where tertiary structural subunits combine to make a functional protein. Hemoglobin is an example of such quaternary structure. 


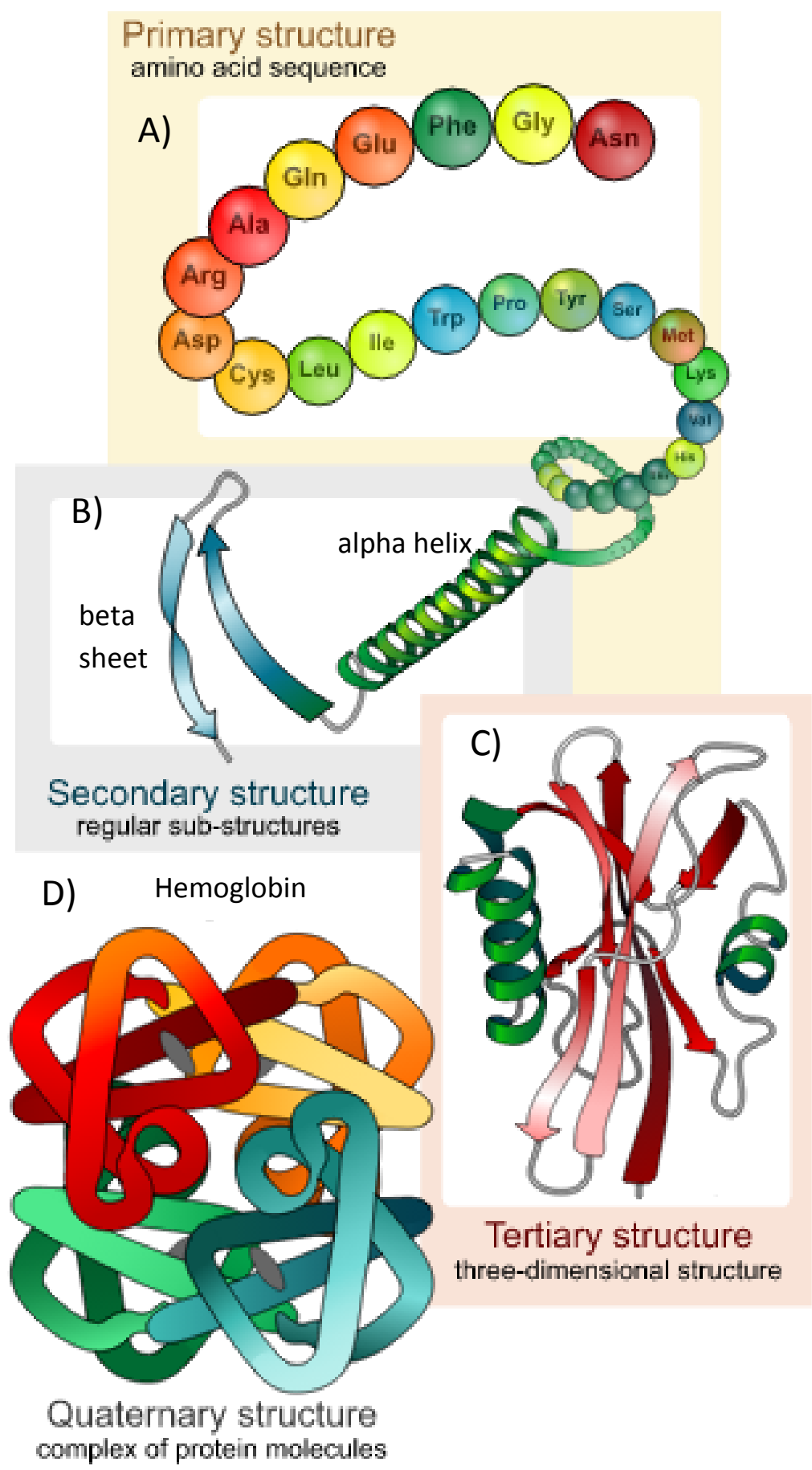

Figure 1.5: The four structural levels of proteins. A) the primary structure; B) common secondary structures of proteins (alpha helix and beta sheets); C) a tertiary structure of a protein (PI3); D) the quaternary structure of hemoglobin. Reproduced from Wikimedia Commons. $^{24}$ 


\subsubsection{Phosphoproteomics}

An important aspect of proteomic research involves the study of post-translational modifications (PTMs): the covalent modification of protein side chains following translation. There are over 400 PTMs listed in the UniProt database of post-translational modifications. ${ }^{25}$ Protein phosphorylation involves the addition of a phosphate moiety to the hydroxyl group of serine, threonine and tyrosine residues. The reverse reaction is known as dephosphorylation, which requires the removal of the phosphate group by hydrolysis. This reversible reaction, illustrated in Figure 1.6, plays an important role in a number of signal transduction pathways in biological systems. It is estimated that approximately $30 \%$ of the human proteome is phosphorylated at a given time ${ }^{26}$ and that phosphorylation or dephosphorylation can be the cause or consequence of a number of human diseases, including cancer and diabetes. ${ }^{26}$ The ratio of phosphorylated serine, threonine and tyrosine residues found in phosphorylated proteins is estimated to be $1800: 200: 1$ respectively; ${ }^{27}$ therefore, a lot of attention is devoted to study potential phosphorylation sites in proteins and peptides. The methods developed in this thesis demonstrated the potential to help identify phosphorylation sites on peptides or proteins of interest. 


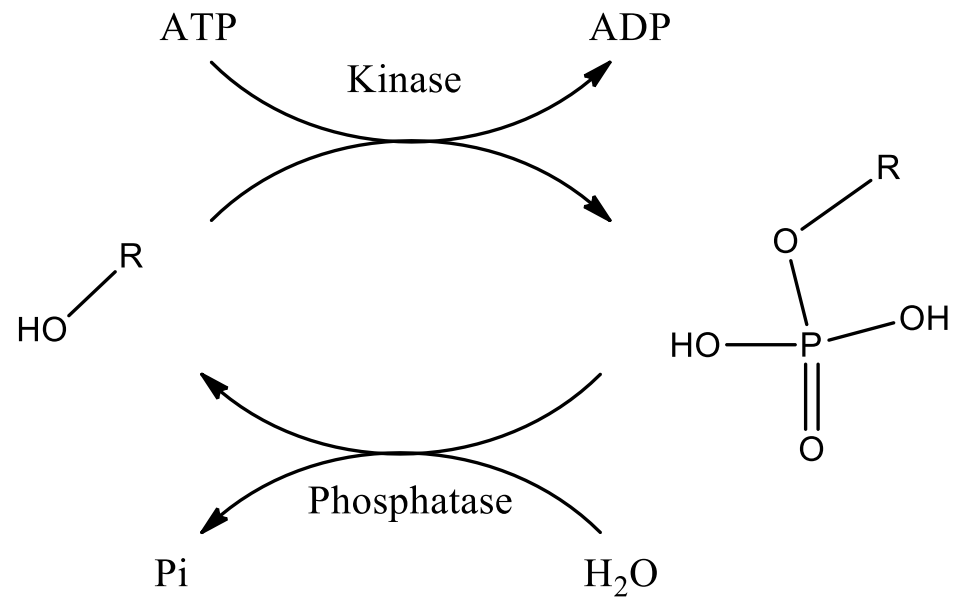

Figure 1.6: Reversible reaction of phosphorylation on $\mathbf{R}$. R implies a serine, threonine or tyrosine residue. 


\subsection{Lipid Biochemistry}

Lipids are commonly defined as substances of biological origin that are soluble in organic solvents. ${ }^{21}$ They are essential small molecules that have roles in a wide variety of physiological functions. ${ }^{8}$ Lipids are the most common biomolecules found in the brain and make up almost half of its dry weight. ${ }^{28,29}$ The role of lipids can be summarized briefly into three major functions: they are essential structural components of cell membranes; they act as energy storage molecules, and play an important role in many intra and intercellular signalling events. ${ }^{8}$ They have also been known to modify proteins post-translationally. ${ }^{30}$ Lipids aggregate together to form macromolecular assemblies or bilayers that are tightly regulated. ${ }^{19}$ Deregulation of lipids can cause a number of physiological problems including the onset of diseases such as cancer, diabetes, Alzheimer's, and other neurodegenerative diseases., ${ }^{8,31}$ In 2005, a classification system for lipids was created to regroup them into eight distinct categories as illustrated in Figure 1.7. The importance of each class of lipid and their roles in biological systems are far beyond the scope of this dissertation. However, a brief description of glycerophospholipids and sphingolipids, as highlighted Figure 1.7 will be presented. 

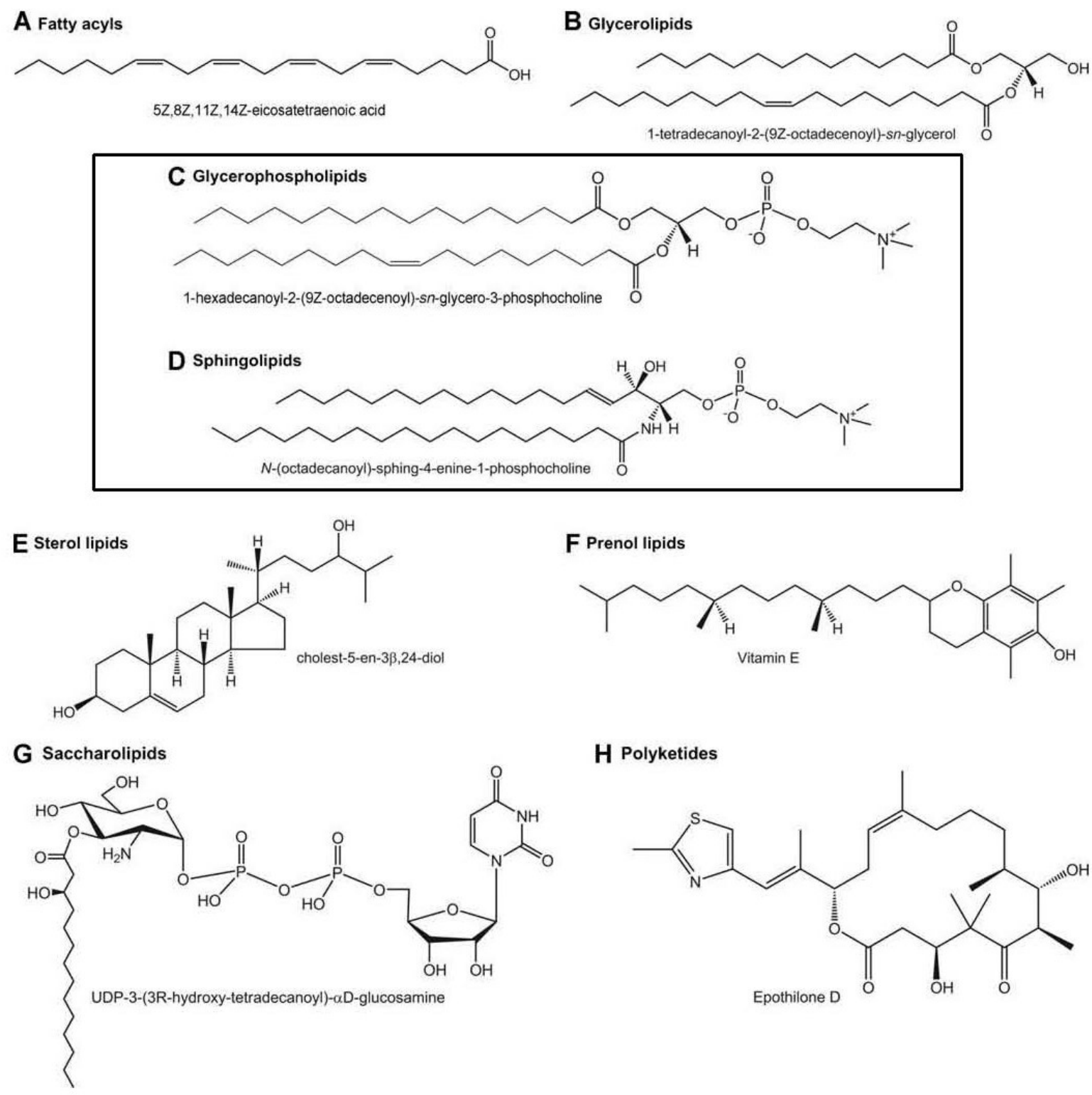

H Polyketides

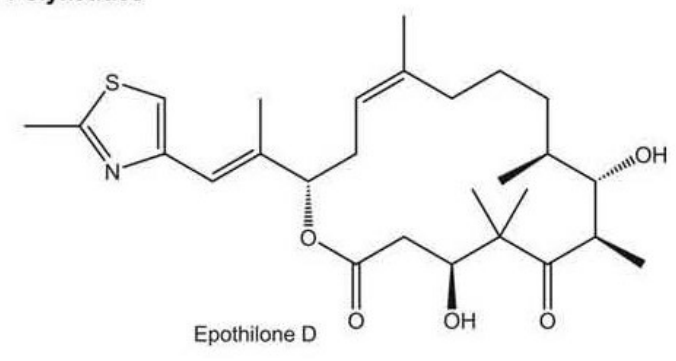

Figure 1.7: Representative structures of the 8 lipid classes. Lipid classes of interest are boxed. Adapted from Bou Khalil et al. 2010.8

Glycerophospholipids are composed of a polar head group at the sn-3 position via a phosphodiester linkage, and acyl esters or ether groups at the $s n-1$ and $s n-2$ positions along the glycerol backbone (Figure 1.8). Glycerophospholipids are subdivided into smaller classes that vary in their polar head groups, including phosphatidylcholines (PC), 
phosphatidylethanolamines (PE) and phosphatidylserines (PS) as highlighted in Figure 1.9. PC, PE and PS are among the most abundant lipids found in mammalian cell membranes. ${ }^{8} \mathrm{PC}$ has a fixed positive charge that is a desirable feature for mass spectrometric analysis. PE is similar to PC in its structure but does not contain the tetraalkylammonium salt structure. PS is functionalized with a serine group on the polar head; at physiological conditions, it contains two negative charges.

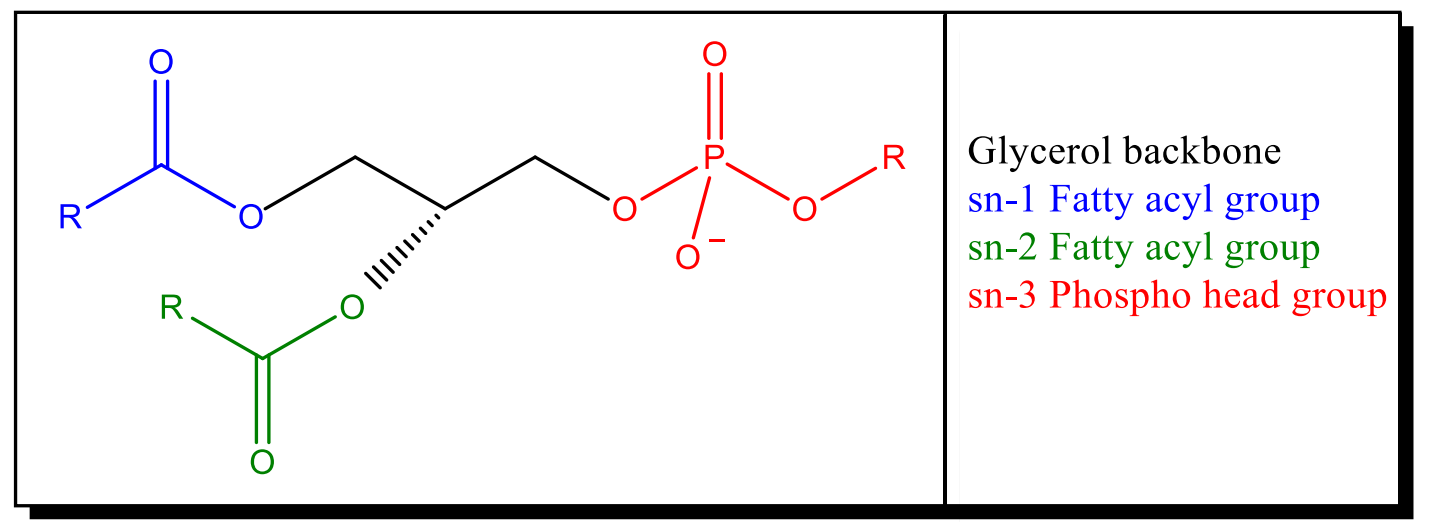

Figure 1.8: The general structure of a glycerophospholipid 


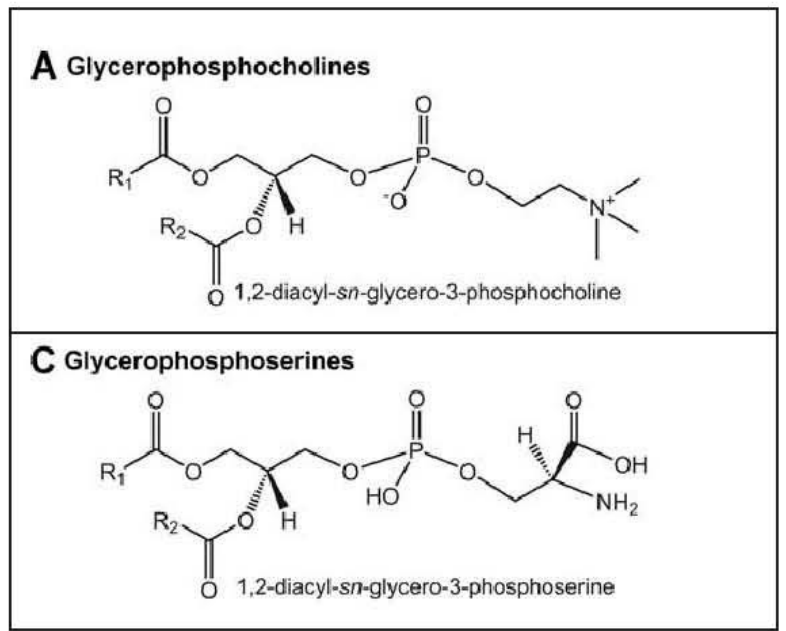

E Glycerophosphoglycerophosphates

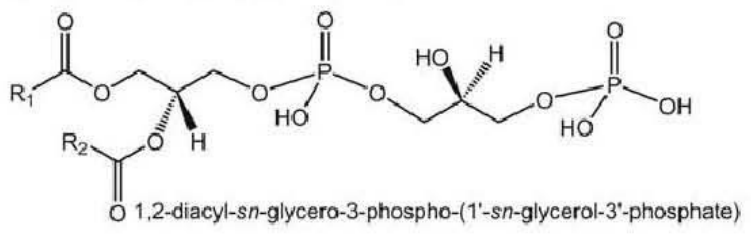

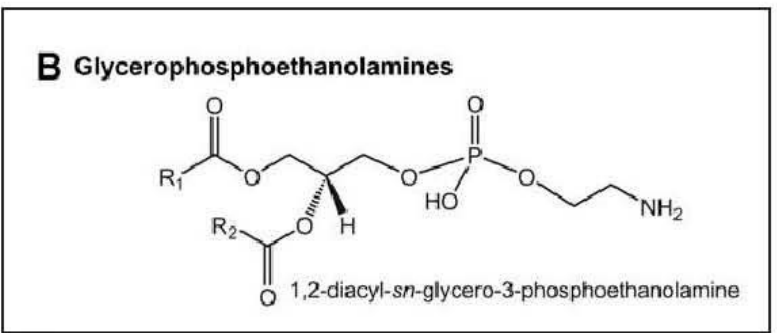

D Glycerophosphoglycerols

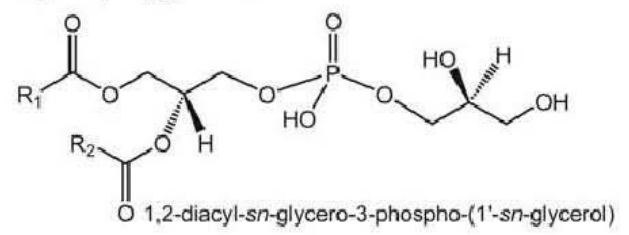

F Glycerophosphoinositols

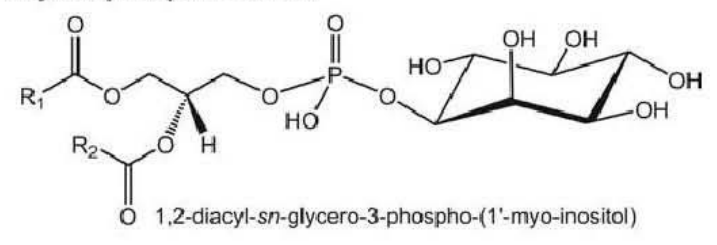

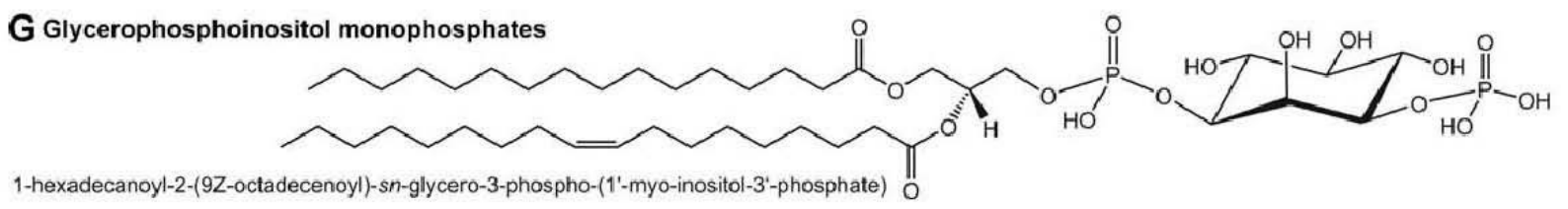

Figure 1.9: The 8 glycerophospholipid subclasses. Those highlighted are of particular relevance to this thesis. Adapted from Bou Khalil et al 2010.8

Sphingolipids are commonly found in neural tissues ${ }^{32}$ and are known to play an important role in signal transduction and cell signaling. ${ }^{8}$ They are derived from the aliphatic amino-alcohol sphingosine, or sphing-4-enine, ${ }^{8}$ as illustrated in Figure $1.10 \mathrm{~A}$. Among the sphingolipids is sphingomyelin (SM) a member of the phosphosphingolipid subclass as highlighted in Figure $1.10 \mathrm{C}$. It has similar properties to PC as it too contains a choline moiety. PC, PE, PS and SM are of interest in this dissertation as they are used to test the efficiency of a novel technique developed to enhance the sensitivity of lipid analysis. 
A. Sphingoid bases

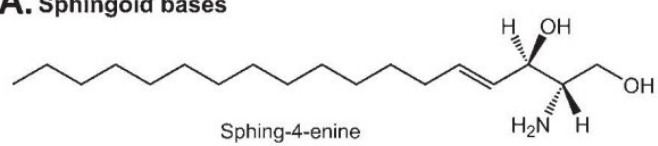

B. Ceramides

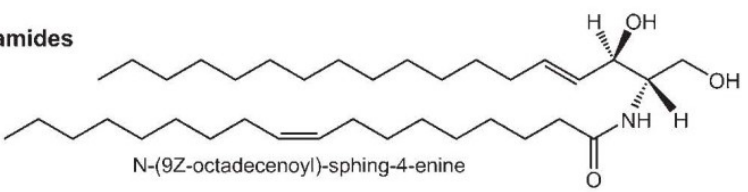

C. Phosphosphingolipids

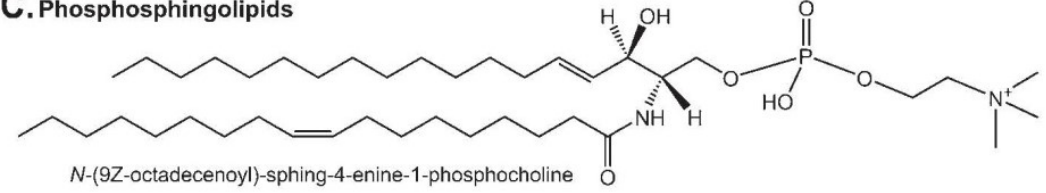

D. Phosphonosphingolipids

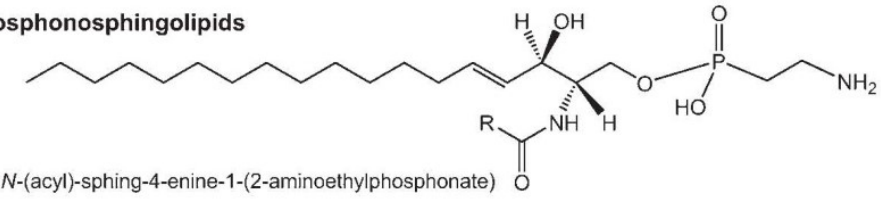

E. Neutral glycosphingolipids

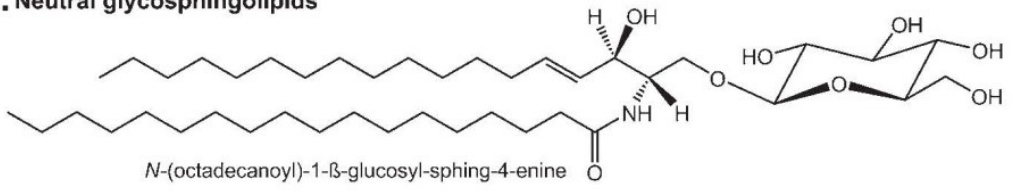

F. Acidic glycosphingolipids

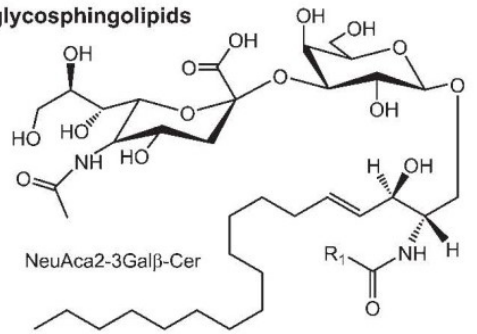

G. Basic glycosphingolipids

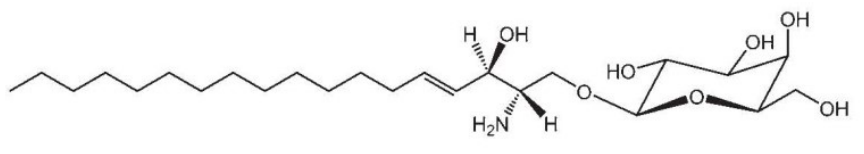

1- $\beta$-galactosyl-sphing-4-enine

H. Amphoteric glycosphingolipids

I. Other sphingolipids
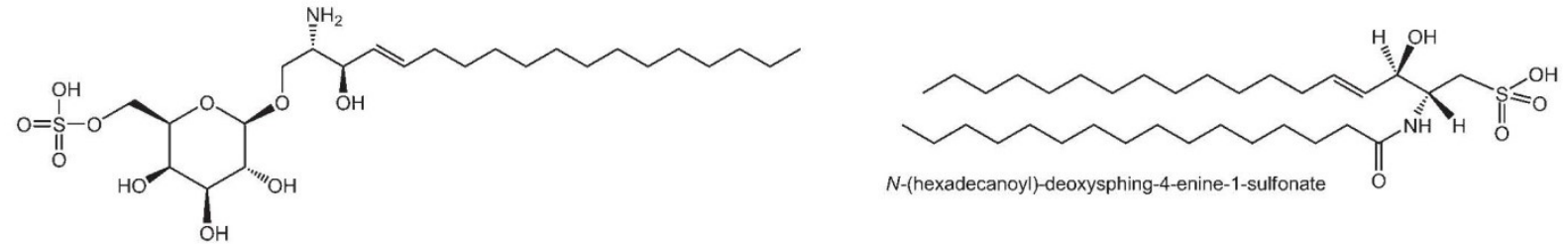

(2S,3R,4E)-2-amino-3-hydroxyoctadec-4-en-1-yl $\beta$-D-galactopyranoside 6-(hydrogen sulfate)

Figure 1.10: Representative structures of sphingolipids. Highlighted is the phosphosphingolipid commonly known as sphingomyelin. Adapted from Bou Khalil et al 2010. ${ }^{8}$ 


\subsection{Mass spectrometry}

Mass spectrometry is a very powerful and versatile instrument. MS traces its roots to the work of Sir J. J. Thomson beginning a century ago and was primarily used by physicists and analytical chemists. Since its birth, numerous advances in the field of MS have occurred allowing it to become one of the most powerful and versatile instruments used today, from the detection of unknown materials, as a diagnostic tool, to dating components of the earth, and for determining the chemical compositions found on other planets. $^{33}$ Despite vast improvements, the functional elements of the mass spectrometer remain the same. Mass spectrometers are typically comprised of three main parts: an ionization source, mass analyzer(s) and a detector (Figure 1.11).

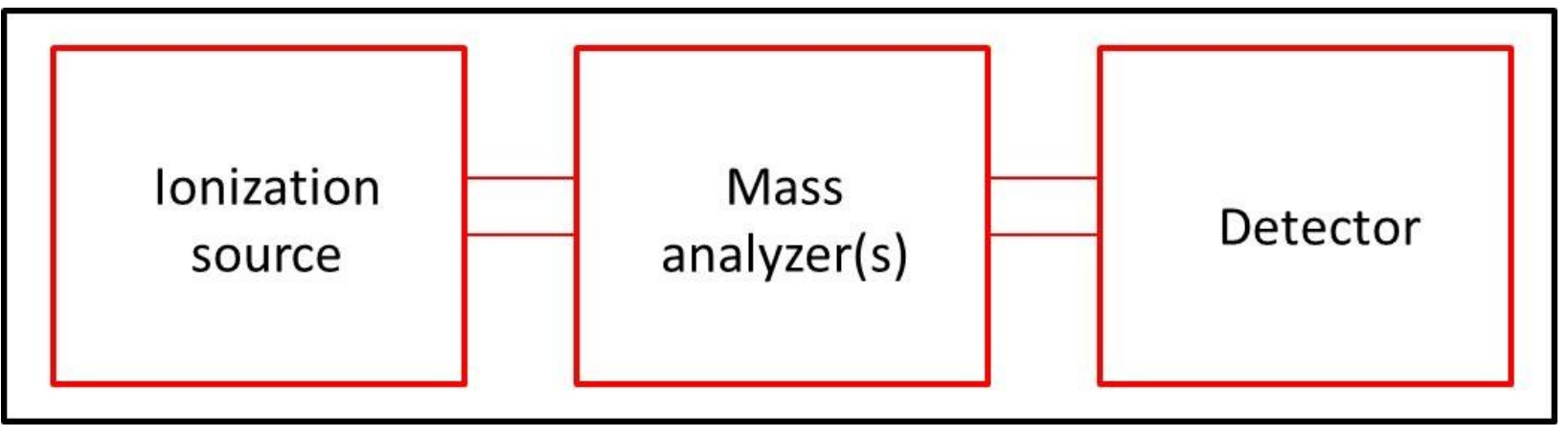

Figure 1.11: General components of a mass spectrometer

Before a mass spectrometer can begin to detect the mass of an analyte, it requires the analyte to be ionized and in the gas phase. Not every analyte is inherently charged or in the gaseous phase, therefore, an ionization source is employed. There are numerous types of ionization techniques tailored for different classes of molecules, such as electron impact 
ionization (El), commonly used for small organic molecules, atmospheric pressure chemical ionization (APCI) for medium-polarity molecules, and soft ionization techniques such as matrixassisted laser desorption ionization (MALDI) and electrospray ionization (ESI) for large biomolecules. While they differ in their methods of ionization, they all produce ions in the gas phase to enable MS analysis.

Mass analyzers are the second essential component to all mass spectrometers. It is the mass analyzer that determines the mass-to-charge ratio $(\mathrm{m} / \mathrm{z})$ of the ions of interest based on the motion of the ions in an electric or magnetic field. The unit for the $\mathrm{m} / \mathrm{z}$ is known as Thomson (Th). ${ }^{34}$ The $\mathrm{m} / \mathrm{z}$ of ions can be separated in space via an ion trap, in time through a time-of-flight instrument, or can be separated through a mass filter known as a quadrupole. Each of these analyzers varies in their sensitivity, mass range and resolution (ability to accurately distinguish between two ions); however, they all are used to enable ion separation. Furthermore, mass spectrometers can have more than one mass analyzer working in tandem to enable a researcher to perform some very unique experiments (vide infra).

The last major component of the mass spectrometer is the detector. The detector is essential for converting ions separated by $\mathrm{m} / \mathrm{z}$ into an electric signal that is ultimately used to produce a mass spectrum. An appropriate detector is selected based on its sensitivity, response time and dynamic range. Common detectors include electron multipliers, channeltrons and the microchannel plate; the latter will be discussed briefly. 


\subsubsection{Electrospray ionization}

Electrospray ionization (ESI) is considered a soft ionization method because of its ability to gently ionize molecules into the gas phase. The technique was invented by Malcolm Dole ${ }^{35}$ and was later adapted by Nobel Prize winner John Fenn ${ }^{4}$ for its use with biological molecules. ESI involves the introduction of an analyte solution at a flow rate of microliters per minute, through a highly charged (positive or negative) needle capillary tip, which charges the analyte solution into a fine spray of charged droplets. ${ }^{36,37}$ The entirety of the work in this thesis is performed on positive ions; therefore, charged ions and positive ions will be equivalent for the remainder of this dissertation. The spray of charged droplets is emitted from the capillary in the form of a jet followed by a plume, also known as a Taylor cone. ${ }^{38}$ The solvent quickly evaporates, concentrating the charge to solvated ions, which causes a Coulombic explosion into smaller droplets. ${ }^{35}$ These small droplets are propelled by an electric field towards the mass spectrometer $^{36,37,39}$ (Figure 1.12).

A variation to electrospray is nanospray: electrospray with flow rates occurring at nanolitres per minute. Nanospray, developed by Smith ${ }^{40}$ and Mann, ${ }^{41}$ is widely used for ionizing biological analytes for MS analysis. Briefly, approximately $20 \mathrm{~nL} / \mathrm{min}$ of solution is delivered through the orifice ( $\sim 1 \mu \mathrm{m}$ diameter) of a capillary made of fused silica. A capillary built for direct infusion is typically coated with $\mathrm{Au} / \mathrm{Pd}$ and used at voltages of $0.8-1.5 \mathrm{kV}$. Nanospray tips facilitate the evaporation of solvent as they produce droplets that are two to three orders of magnitude smaller than conventional electrospray. ${ }^{42}$ Furthermore, electrospray 
and nanoelectrospray can be coupled for online operation with HPLC, though these are usually uncoated fused silica and require higher voltages.

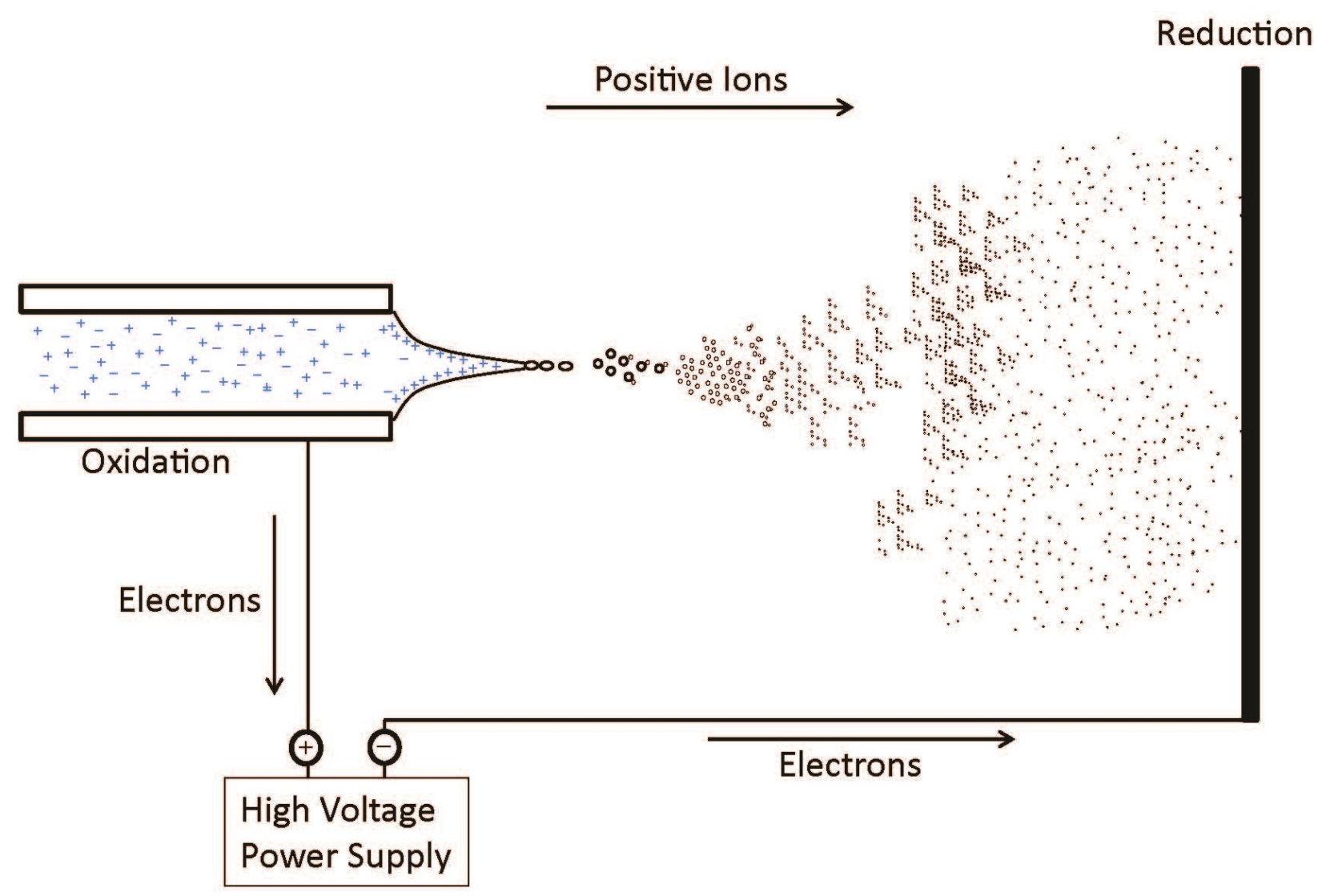

Figure 1.12: General overview of electrospray ionization. The reduction electrode would have an orifice so that ions may enter the mass spectrometer. 


\subsubsection{Quadrupole mass analyzers}

Quadrupoles consists of four parallel, electrically-connected metal rods that have constant and radio frequency (RF) voltages applied to them. ${ }^{43}$ Potentials are applied to the rods in order to facilitate the trajectory of the ions towards the detector. Briefly, a positive potential is applied to two electrodes opposite each other, while a negative potential is applied to the other two electrodes. Therefore, ions entering the quadrupole will experience an electric field in both the $x-z$ and $y-z$ planes due to the potentials on the rods (Figure 1.13). The simultaneous application of both positive and negative potentials to the rods allows for selective transmission of ions through the quadrupole.

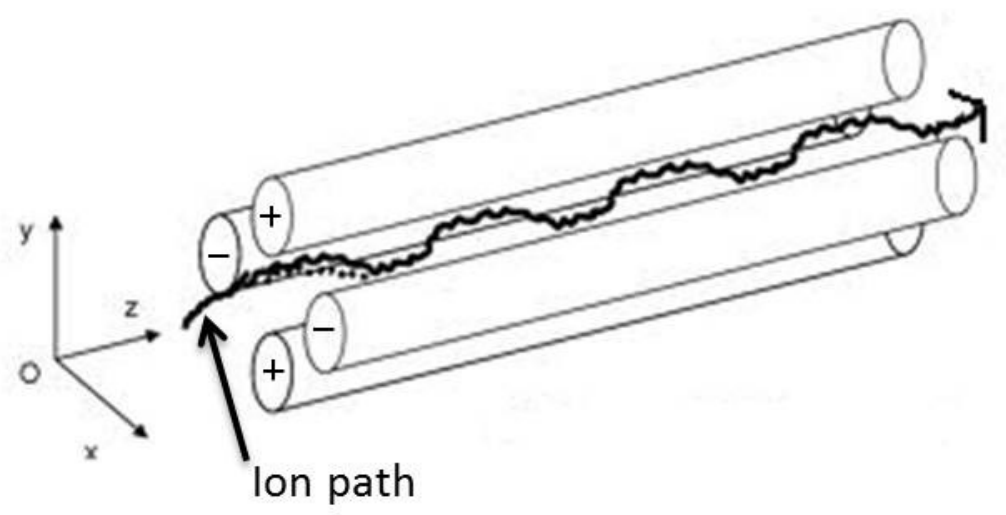

Figure 1.13: Applied voltages to quadrupole rods

To select a bias towards heavier ions, a positive direct current (DC) potential is applied such that the overall potential in the $x-z$ plane rods are positive. This guides the heavier ions towards the center axis of the rods and towards the detector, while forcing lighter ions to 
accelerate out of the trajectory to eventually become neutralized by the rods. On the contrary, when an overall negative DC potential is applied on the $y$-z plane rods, it will force heavier ions to diverge from the center axis of the rods due to their high momentum, while the light ions will be guided back towards the center axis. Rapidly varying voltages are used to select ions of different masses to be guided to the detector. The quadruple, therefore acts as a narrow bandpass filter such that at a given time, only a selected range of ions will have stable enough trajectories to pass through the rods. Figure 1.14 illustrates the range of ions that will have stable trajectories to be transmitted through the quadrupole at a given time. The width of the shaded triangle in Figure 1.14 determines the resolution of the mass filter; the slimmer the width of the triangle, the greater the resolution and vice versa. Should a slimmer bandpass be desired, it can be achieved by increasing the DC potential relative to the RF potential though it would reduce the sensitivity of the quadrupole (decrease in height of the triangle). This narrow bandpass filter can sequentially allow successful transmission of ions with increasing $\mathrm{m} / \mathrm{z}$ values by sweeping through a range of voltages. Quadrupoles typically cover a range up to 4000 $\mathrm{m} / \mathrm{z}^{43}$

An additional operational mode for quadrupoles is the RF only mode. RF only mode allows a large number of ions to have stable trajectories with successful transmission through the quadrupole. This is an important feature in many mass spectrometers when more than one mass analyzer is combined in a single instrument (vide infra). 


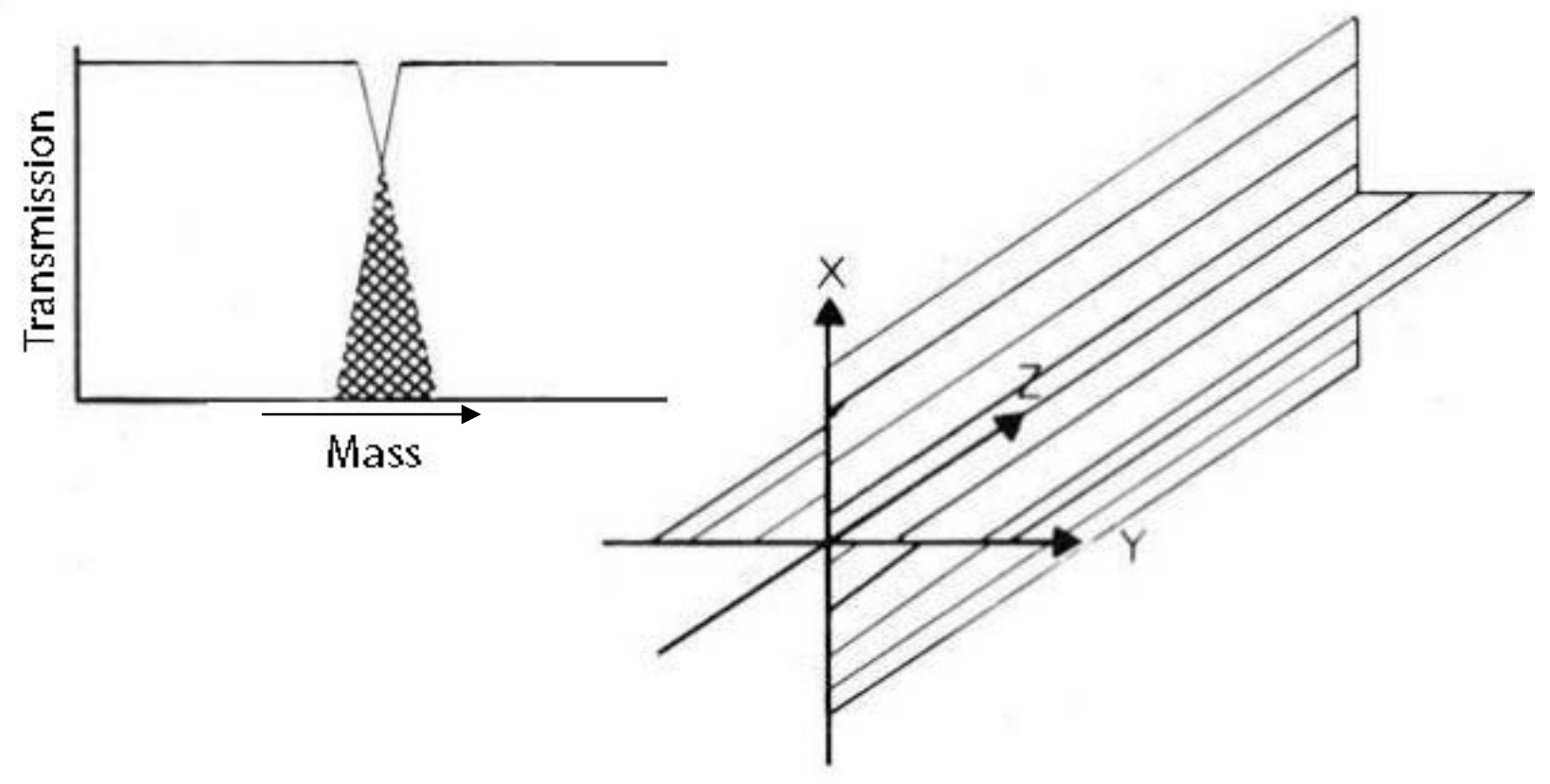

Figure 1.14: High-bandpass filtering in the xz plane and low-bandpass filtering in the yz plane. Adapted from Miller et al. ${ }^{34}$

\subsubsection{Time-of-flight}

Time-of-flight (TOF) mass analyzers function under high vacuum ( $10^{-7}$ Torr) and separates ions based on the time it takes for an ion to travel a fixed distance. Essentially, heavier ions will take longer than lighter ions to reach their final destination when they are both subjected to the same electric field. Figure 1.15 illustrates the simplest TOF geometry, where three ions are accelerated by the same electric field in an ion accelerator. In this case, the lighter ions (light $\mathrm{m} / \mathrm{z}$ ) will have a higher velocity than the heavier ions (heavy $\mathrm{m} / \mathrm{z}$ ) and will therefore reach the final destination (detector) in a shorter time. However, this simple representation of a TOF works best if the initial kinetic energy of the ions entering the ion 
accelerator, the initial direction of the velocity and the spatial distribution of the ions in the ion accelerator region are the same. In reality, these three conditions may not always be met. To correct for these three conditions, an orthogonal injection TOF instrument with reflecting geometry can be used. ${ }^{44,45,46}$

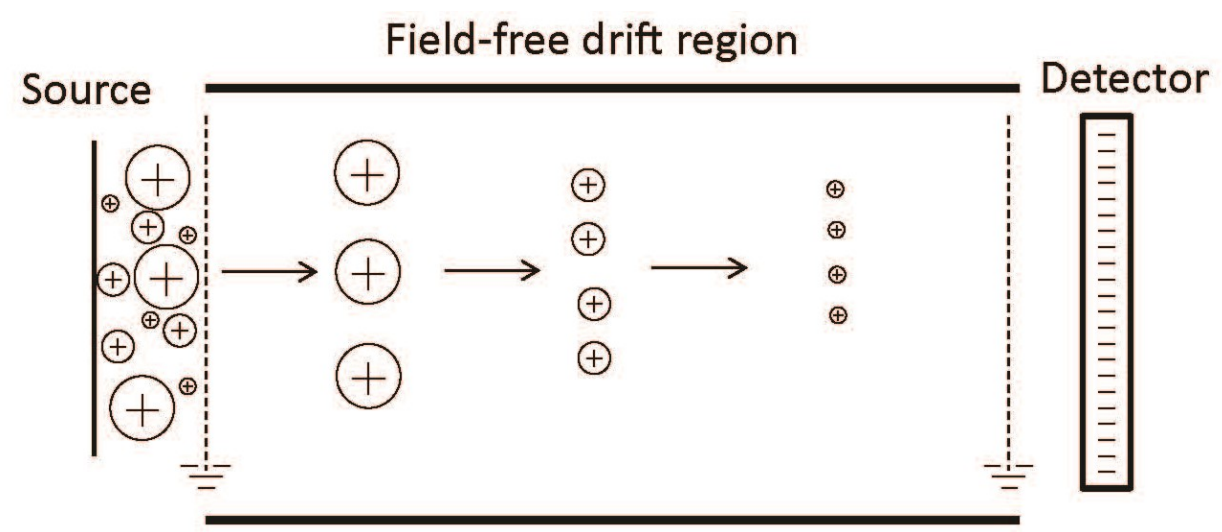

Figure 1.15: General schematic of time-of-flight mass spectrometry

A TOF mass spectrometer with orthogonal ion injection uses a continuous ion source (such as ESI), an ion modulator, and a drift region followed by a detector. The ion source provides a continuous flow of ions towards the modulator (or ion accelerator), though without collisional cooling or focusing with ion optics, ions may diverge from their initial path and may have a larger variation in their velocity distribution. ${ }^{47}$ Collision cooling, or collisional damping uses the quadrupole in RF mode with higher pressure to induce collisions with molecules to reduce their velocity prior to entering the ion accelerator. ${ }^{48}$ This produces a beam with a smaller spatial and velocity spread. ${ }^{47}$ Furthermore, a reflectron, or ion mirror, not only doubles the drift tube length thereby increasing the resolution of the mass spectrometer, but it is also used to correct for small differences in velocity of ions with the same $\mathrm{m} / \mathrm{z}$. A small ion that was 
accelerated in the ion accelerator faster than another ion of the same $\mathrm{m} / \mathrm{z}$ will proceed further into the ion mirror and vice versa. Figure 1.16 demonstrates how ions with the same $\mathrm{m} / \mathrm{z}$ and different accelerations are corrected by the ion mirror so that they may reach the detector at the same time. Unlike quadrupoles, TOF mass analyzers do not have a theoretical upper mass limit; therefore making them appropriate for detecting both small and large biomolecules.

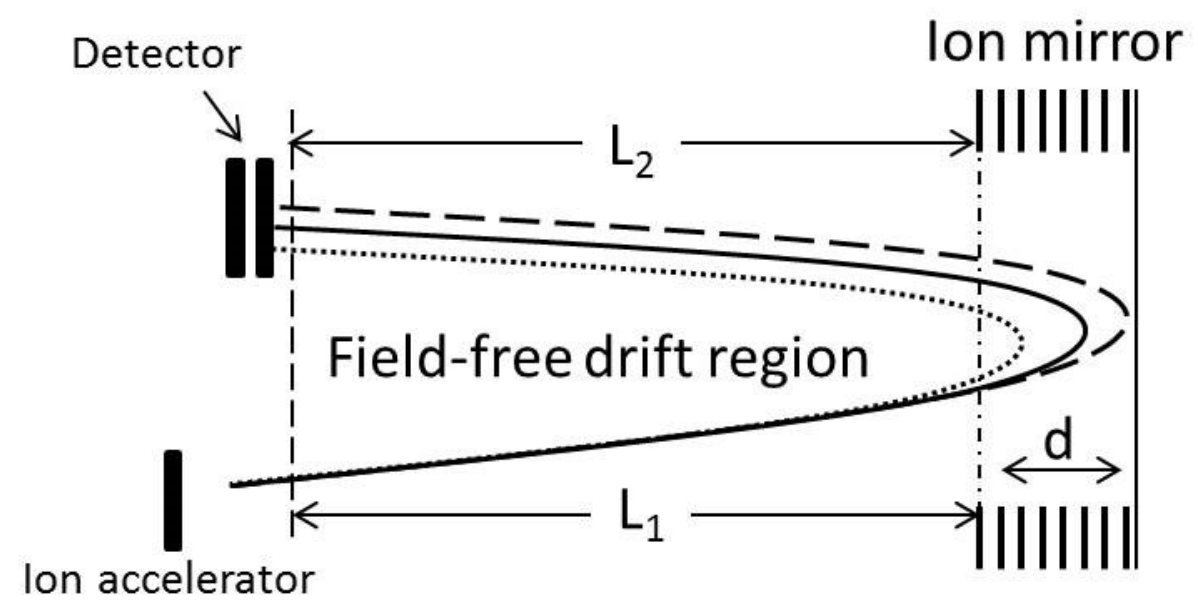

Figure 1.16: Ion mirror correcting the same $\mathrm{m} / \mathrm{z}$ ion with different velocities 


\subsubsection{Ion detectors}

Typically, detectors involve the collision of the mass-separated ions onto a surface, inducing a measurable signal that is proportional to the number of impinging ions. ${ }^{36}$ The most common detector used in this work was the microchannel plate (MCP) detector. An MCP detector is composed of an array of miniature channel electron multipliers (CEMs). Mass spectrometers typically have two MCPs in a chevron configuration to maximize the signal generated from an ion as illustrated in Figure 1.17. Briefly, a single ion impingement releases a detectable cascade of electrons down one of the channels in the first MCP. When the cascade of electrons exits the first $\mathrm{MCP}$, they spread radially causing multiple electron cascades in multiple CEMs of the second MCP. These cascades of electrons eventually reach a multi-anode $\operatorname{array}^{49}$ that is connected to a sensitive current meter and a signal is recorded.

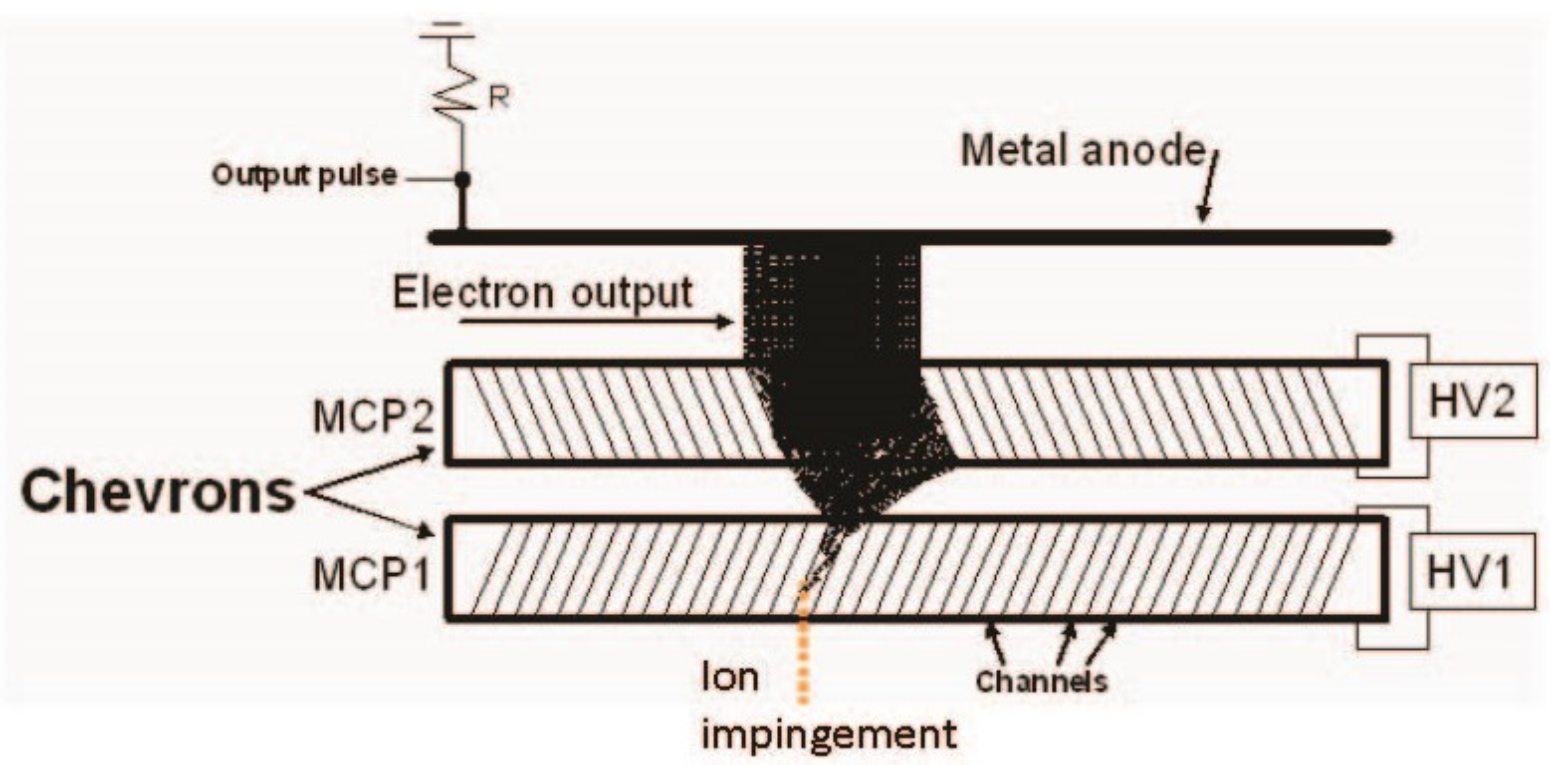

Figure 1.17: Microchannel plate detector. Ion impingement releases a cascade of electrons towards the metal anode, where a signal is recorded. Reproduced from Smith. ${ }^{36}$ 


\subsubsection{Hybrid quadrupole-time-of-flight mass spectrometer}

The main instrument used throughout this thesis is the hybrid quadrupole-time-of-flight mass spectrometer known as the Qstar XL from AB Sciex. It is abbreviated as a QqTOF, where Q describes a mass resolving quadrupole and $q$ describes an RF only quadrupole. A schematic diagram of the QqTOF is illustrated in Figure 1.18. Briefly, the sample is ionized by ESI at atmospheric pressure, stripped into bare ions via gentle collisions with a curtain gas ${ }^{50}$ and passed through a charged orifice into a vacuum chamber allowing for the gaseous ions to expand supersonically ${ }^{36,51}$ (Figure 1.19).

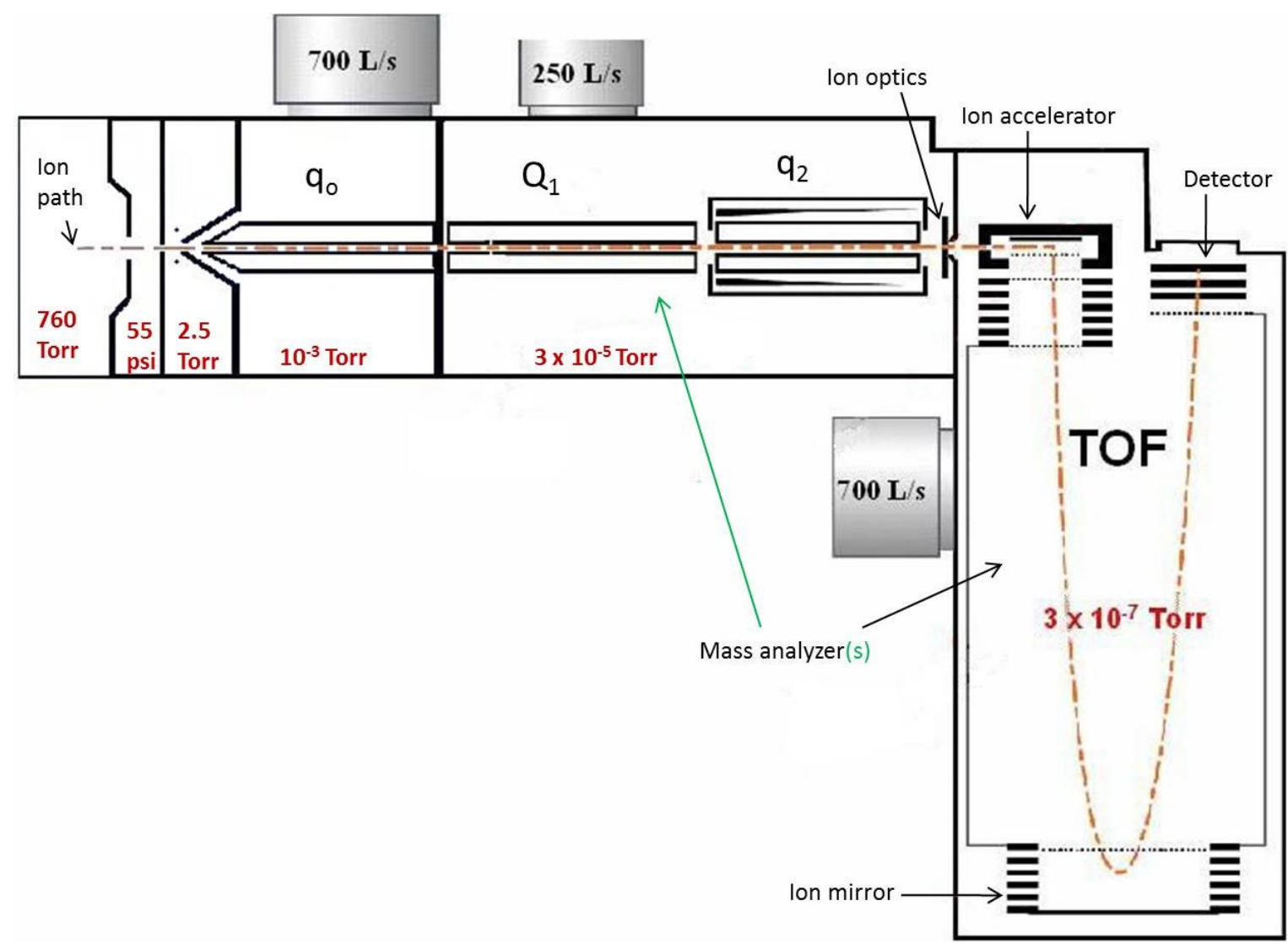

Figure 1.18: General schematic of a QqTOF mass spectrometer. Adapted from Smith. ${ }^{36}$ 


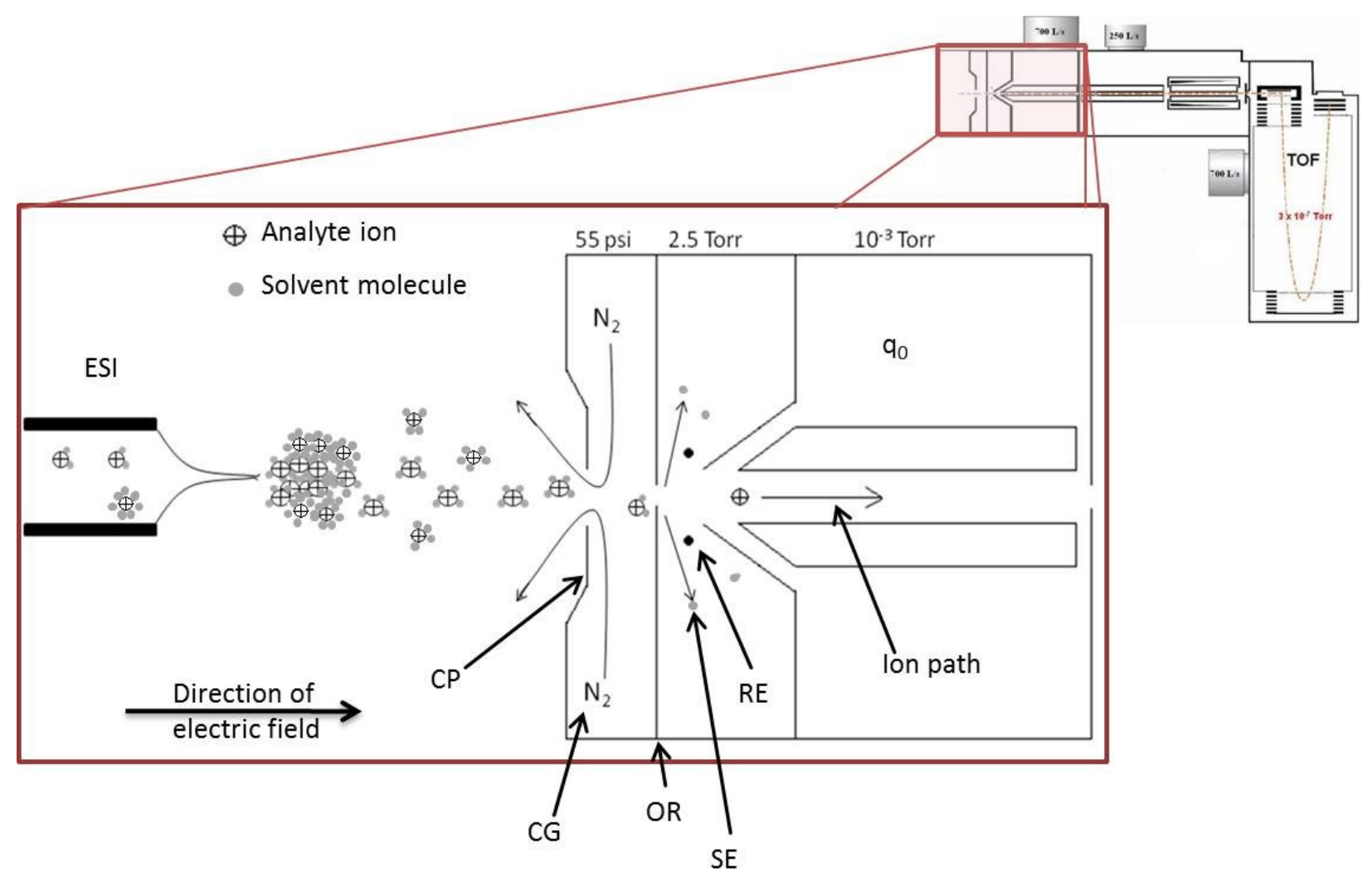

Figure 1.19: Sample ions produced by ESI are being introduced to the front end of the QqTOF. "CP" refers to curtain plate; "CG" refers to curtain gas; "OR" refers to orifice; "SE" refers to supersonic expansion; "RE" refers to ring electrode. Adapted from Smith. ${ }^{36}$

A ring electrode directs the charged ions into the ion path of $q_{0}$ providing collisional cooling ${ }^{52}$ and the guidance of ions into $Q_{1}$ prior to entering $q_{2}$. $Q 1$ can allow the successful transmission of a range of ions or act as a mass filter allowing ions with a set $\mathrm{m} / \mathrm{z}$ to have successful transmission onto $\mathrm{q}_{2}$; this is desirable for product ion scanning (vide infra). The $\mathrm{q}_{2}$ quadrupole is unique in that it acts as a collision cell. Here ions are subjected to an inert gas such as argon, to either slow down the ions via collisional cooling, or to subject the ions to high energy collisions that cause the ions to fragment into smaller daughter ions. This form of fragmentation is known as collision induced dissociation (CID) and is an important feature as it allows for product ion scanning or $\mathrm{MS}^{2}$ analysis. Product ion scanning is among the most 
frequently used scanning modes in tandem mass spectrometers. In this experiment, an ion with a set $\mathrm{m} / \mathrm{z}$ value can be selected with the first mass analyzer $\left(Q_{1}\right)$. The selected ion is permitted to travel into $q_{2}$ to be collisionally activated to induce fragmentation. The fragment ions, also known as daughter ions, are then analyzed with the second mass analyser (TOF). This particular experiment is quite useful as it allows one to acquire fragmentation data that can provide information on the structure of the parent molecular ion. $\mathrm{MS}^{2}$ analysis was most frequently used on the Qstar XL.

After passing through $\mathrm{q}_{2}$, the parent/daughter ions are again collisionally cooled prior to entering the ion modulator of the TOF. Ions are then separated in the TOF as previously described and are detected via the MCP detector (Figure 1.20). Vacuum chambers throughout the mass spectrometer are at appropriate pressures to maintain proper ion trajectories and control ion-molecule collisions. ${ }^{36}$ 


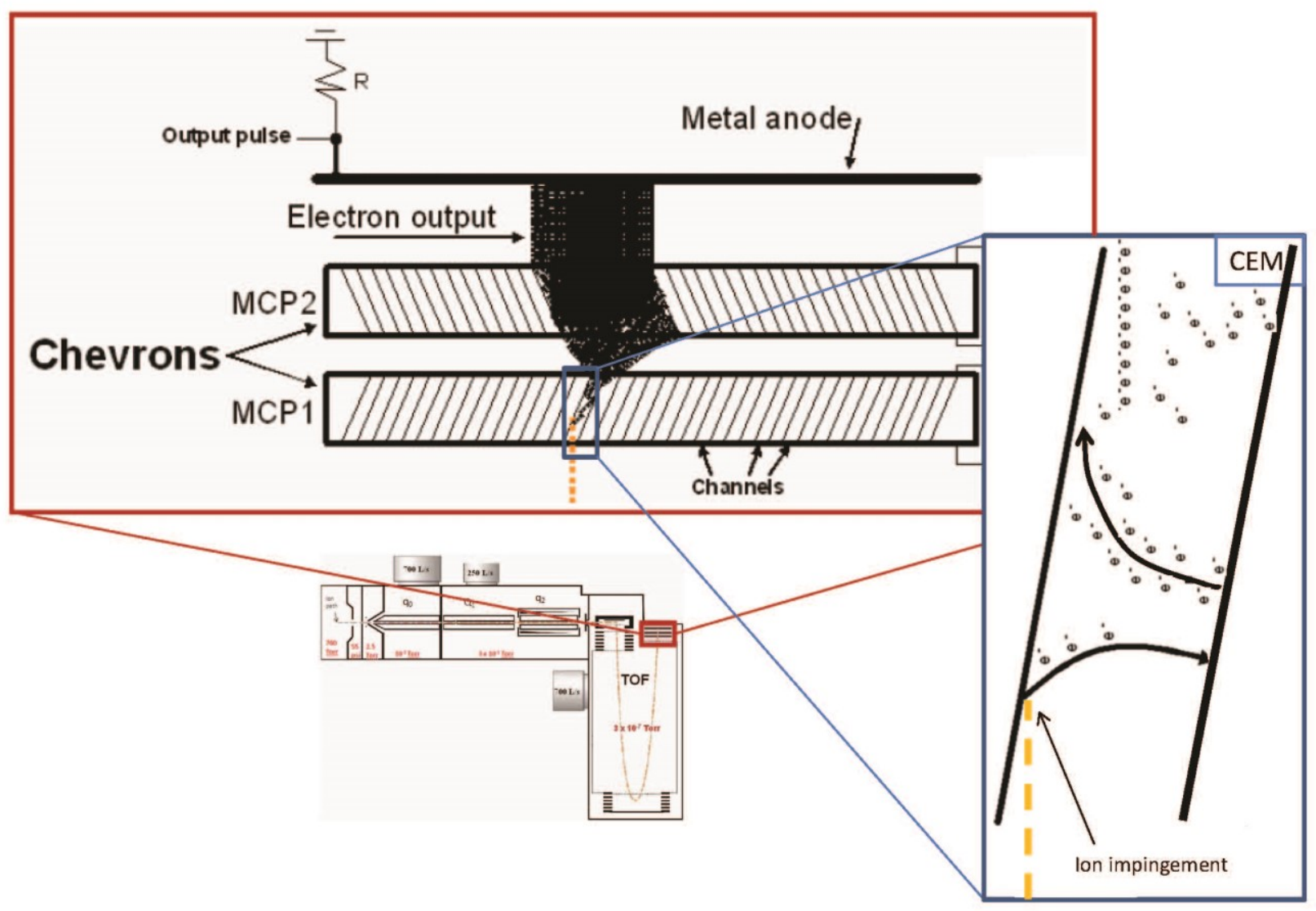

Figure 1.20: The detector of the QqTOF mass spectrometer. Adapted from Smith. ${ }^{36}$ 


\subsubsection{Hybrid triple quadrupole/linear ion trap}

Another instrument used throughout this thesis is the 4000 hybrid triple quadrupole linear ion trap mass spectrometer known as the 4000 Qtrap from $A B$ Sciex. The triple quadrupole is setup in a linear assembly of three quadrupoles as illustrated in Figure 1.21. Though it has three quadrupoles, only the first $\left(Q_{1}\right)$ and third $\left(Q_{3}\right)$ are mass analyzers. The second quadruple $\left(q_{2}\right)$ operates in RF only mode, effectively allowing ions of every mass to be transmitted further. It also acts as a collision cell. Here ions are subjected to an inert gas such as nitrogen to subject the ions to CID. $Q_{1}$ and $Q_{3}$ can be operated with a combination of both RF and DC potentials to perform a wide variety of mass analyzing modes including product ion scanning, precursor ion scanning, neutral loss scan and multiple reaction monitoring. These methods will be discussed in detail.

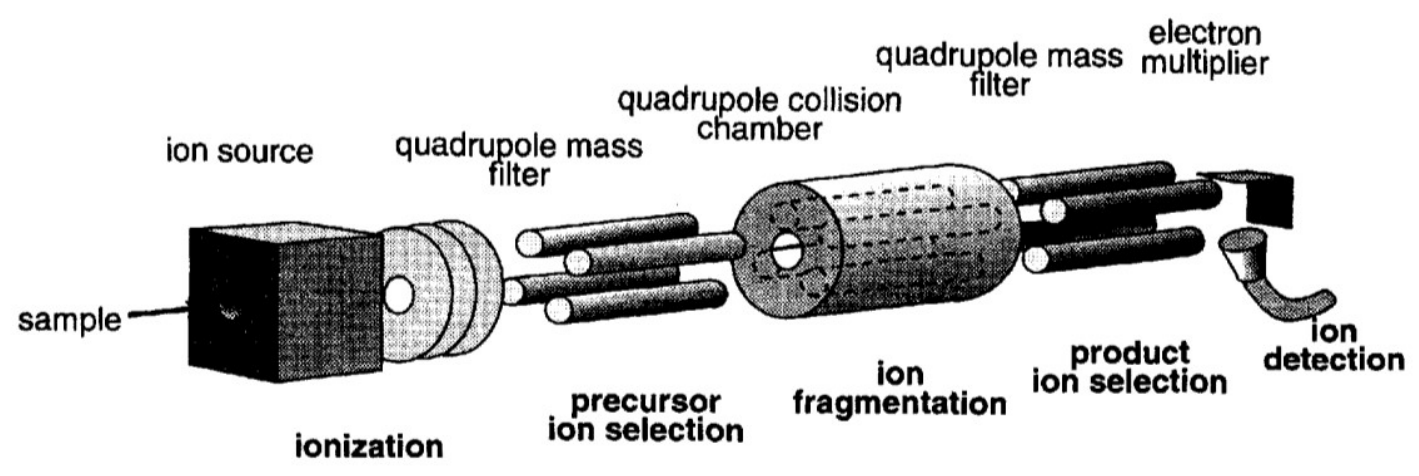

Figure 1.21: General schematic diagram of a triple quadrupole mass spectrometer. Reproduced from de Hoffman. ${ }^{53}$ 
Product ion scanning in the 4000 Qtrap works essentially the same as in the Qstar XL, except that the $2^{\text {nd }}$ mass analyzer is a quadrupole. The Qtrap offers other scanning modes that are of particular interest for this dissertation; among them is precursor ion scanning. In the precursor ion scanning mode, also known as parent scanning, the first mass analyzer $\left(Q_{1}\right)$ is set to allow a range of ions to be transmitted into $q_{2}$ for CID. Ions that pass through $Q_{1}$ will be detected if, and only if, after fragmentation in $q_{2}$ it produces a pre-selected product ion. ${ }^{53}$ This pre-selected product ion with a set $\mathrm{m} / \mathrm{z}$ is the only ion permitted to transmit through the second mass analyzer to the detector. This scan is particularly useful for the selective detection of ions containing a particular functional group of interest. For example, if the second analyzer is set on $\mathrm{m} / \mathrm{z} 184$ (the $\mathrm{m} / \mathrm{z}$ of the phosphocholine head group from $\mathrm{PC}$ ), the precursor ion scan will provide a record of all the ions containing the phosphocholine head group.

The neutral loss scan is a form of functional group-selective scan, much like precursor ion scanning, though it is more complex. The neutral loss scan requires that both $Q_{1}$ and $Q_{3}$ are scanned together with a constant $\mathrm{m} / \mathrm{z}$ difference between the two mass analyzers. For example, a phosphoserine lipid with various chain lengths is ionized and allowed to be transmitted through $Q_{1}$ into the collision cell $\left(q_{2}\right)$ where it fragments. PS will lose its head group, equivalent to $185 \mathrm{Da}$, upon collisional activation; therefore $Q_{3}$ scans a range of ions that passed through $Q_{1}$ and lost a neutral fragment of $185 \mathrm{Da}$. This scan therefore, recognizes all ions, which by fragmentation, lead to the loss of a given neutral fragment.

The last major scanning mode is the multiple reaction monitoring (MRM) mode, also known as selected ion monitoring. In MRM mode, both $Q_{1}$ and $Q_{3}$ are set to select a particular 
ion. Briefly, $Q_{1}$ allows only one parent ion to be transmitted to the collision cell. After fragmentation, only one pre-selected fragment is allowed to be transmitted through $\mathrm{Q}_{3}$ to be detected. This scanning mode is arguably the most sensitive because both mass analyzers are filtering out all other masses, while allowing only one ion each to be guided through to the detector. This particular scanning mode does require prior knowledge to the analyte of choice, including the fragmentation pattern of each analyte. The presence of ions determined by MRM does not produce a mass spectrum, but a chromatogram of intensity versus time. An example of MRM analysis is illustrated in Figure 1.22. Figure 1.23 illustrates the variety of scanning modes.

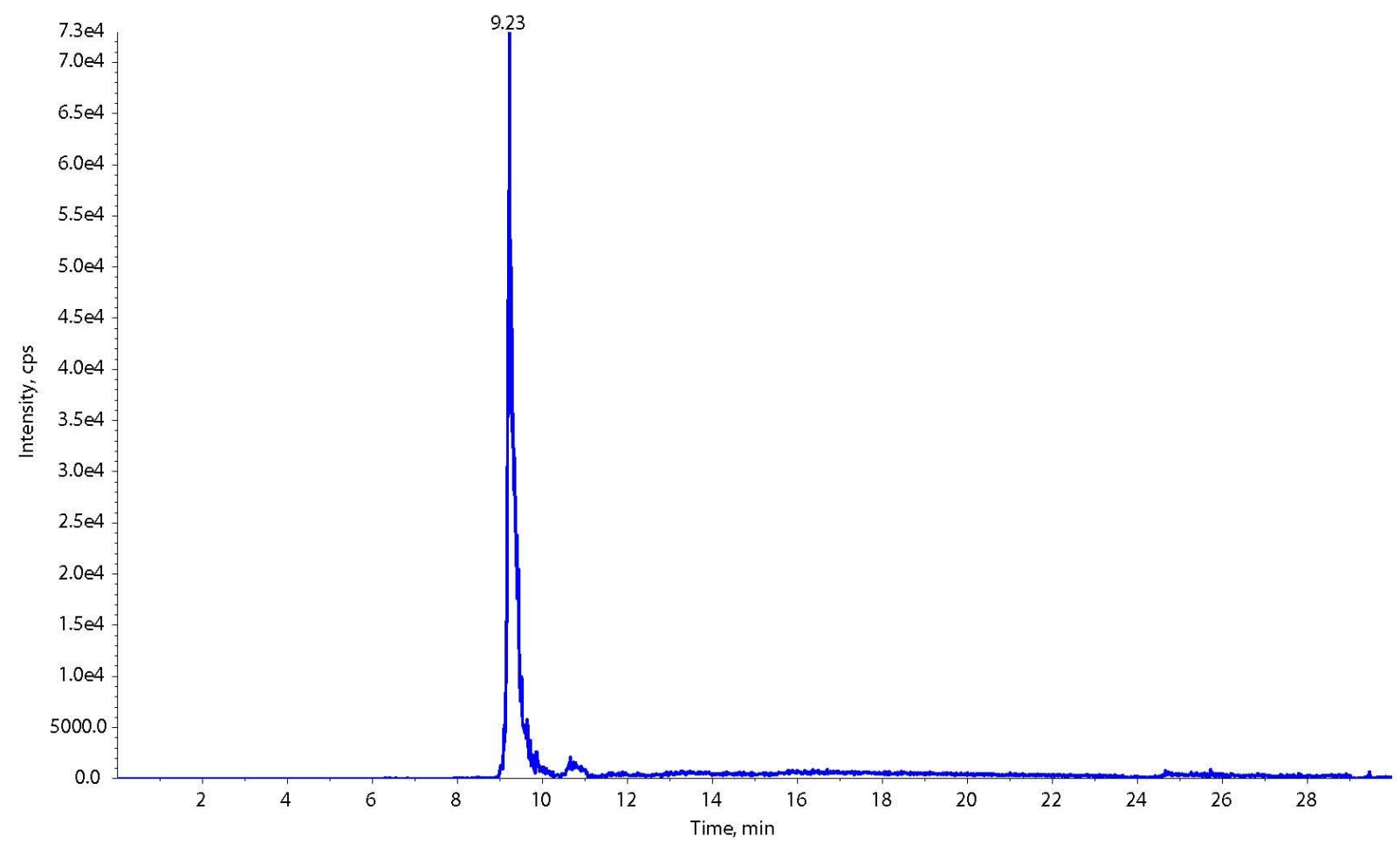

Figure 1.22: Example of a chromatogram obtained from MRM analysis 


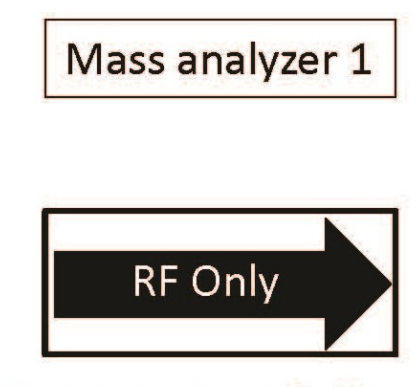

Transmission of all ions

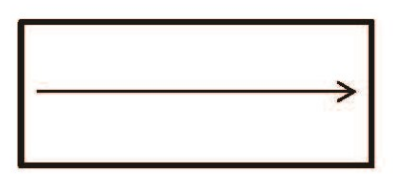

Selected $\mathrm{m} / \mathrm{z}$

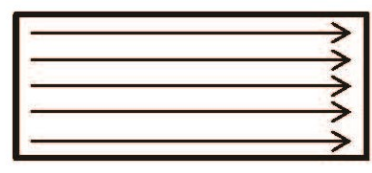

Scanned $m / z=x$

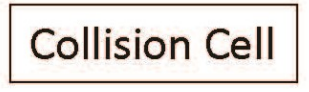

Mass scan

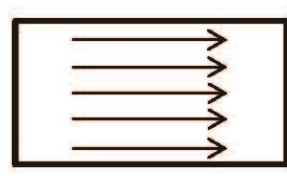

No CID

Product lon Scan

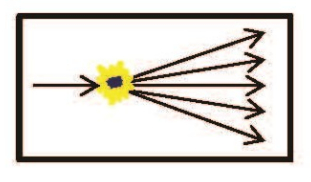

CID

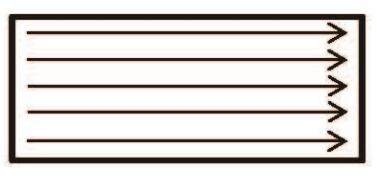

Scanned
Mass analyzer 2

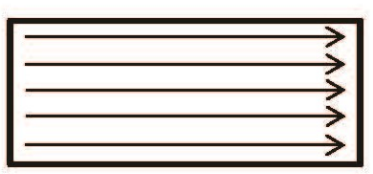

TOF or EMS Scanned

Neutral loss Scan

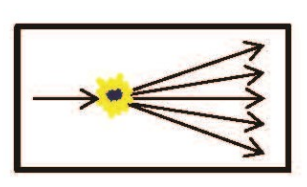

CID

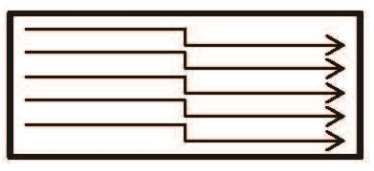

Scanned $m / z=x-a$

Precursor Ion Scan

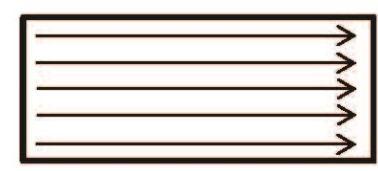

Scanned

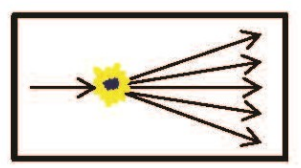

CID

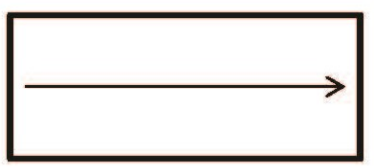

Selected $\mathrm{m} / \mathrm{z}$

Multiple Reaction Monitoring

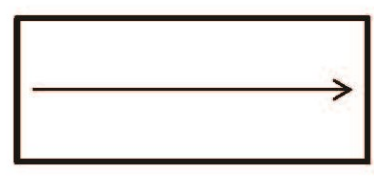

Selected $\mathrm{m} / \mathrm{z}$

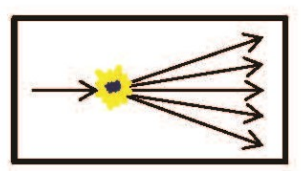

CID

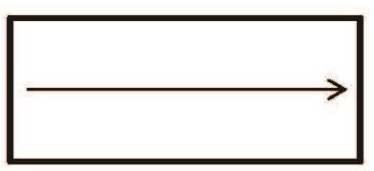

Selected $\mathrm{m} / \mathrm{z}$

Figure 1.23: $\mathrm{MS}$ and $\mathrm{MS}^{2}$ experiments. Between the mass analyzers is a collision cell containing an inert gas used to induce fragmentation. 


\subsubsection{Mass spectrometry based proteomics}

There are two distinct methods to perform mass spectrometry based proteomics: topdown and bottom up proteomics. Top-down proteomics refers to analysis of intact proteins, performing MS/MS on entire proteins. Bottom-up proteomics involves proteolytic digestion of proteins, which converts them into smaller peptides prior to analysis. This method is often preferred as it produces peptides with smaller $\mathrm{m} / \mathrm{z}$ values, less charge distribution and provides easier CID analysis to provide a better sequence coverage of the individual peptides within a protein. Trypsin is a proteolytic enzyme commonly used to digest proteins. Trypsin cleaves the peptide bonds on the C-terminal side of all lysine and arginine residues, unless they are immediately followed by a proline residue.

When a peptide is subjected to CID, the peptide will experience enough internal vibrational energy along the peptide backbone such that covalent bonds may break ${ }^{54}$ through the lowest energy pathways. ${ }^{39}$ Most commonly, the covalent amide bond will break to yield $b$ and $y$-ions. Since mobile protons move freely along the backbone, $\mathrm{CID}$ can produce a series of b- and $y$-ions, providing one with the ability to sequence the entire peptide. There are also less common ions such as $a-, c-, x-$ and $z-$ ions. Figure 1.24 illustrates that $a-, b-$ and $c-$ ions are numbered sequentially from the $\mathrm{N}$-terminus to the $\mathrm{C}$-terminus, while the $\mathrm{x}$-, $\mathrm{y}$ - and $\mathrm{z}$ - ions are numbered sequentially from the $\mathrm{C}$-terminus to the $\mathrm{N}$-terminus. 


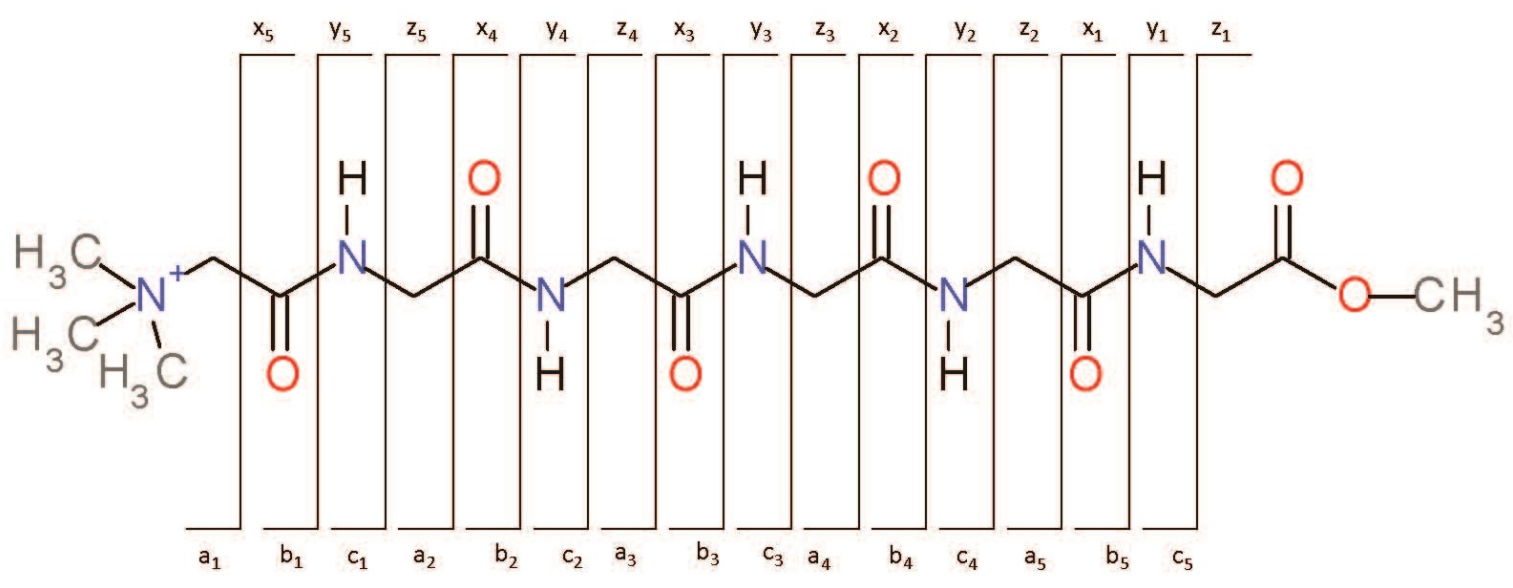

Figure 1.24: Formation of a-, b-, c- and x-, y- and z- ions from fragmentation of tetramethylated hexaglycine. ${ }^{62}$

Protein identification via mass spectrometry is typically coupled to reverse phased HPLC. Coupling liquid chromatography to mass spectrometry for bottom-up proteomic research reduces the complexity of the peptide mixture being injected to the mass spectrometer for analysis. This is advantageous as peptides are eluted in relatively narrow chromatographic windows, therefore allowing an opportunity for lower proton affinity peptides to be identified, though this is unlikely to happen if several peptides with high proton affinities are eluted simultaneously. MS and $\mathrm{MS}^{2}$ analysis via bottom-up proteomics typically uses an informationdependent acquisition (IDA) method. Briefly, MS is continuously recorded, and the software is programed to perform $\mathrm{MS}^{2}$ analysis on all ions with $2+$ (or more) charges and that have signal intensities over a certain threshold. Once the information is acquired, proteins are identified by aligning the measured $b$ - and $y$ - ions in the $M S^{2}$ data to a list of expected masses from each peptide in a given database; identified peptides are mapped to a library of proteins for 
identification. The library is often comprised of protein sequences obtained through predicted genomic information of the biological system in question. ${ }^{8}$

\subsubsection{Mass spectrometry based lipidomics}

Lipids are commonly analyzed by ESI mass spectrometry via direct infusion ("shotgun" lipidomics) or coupled with front-end liquid chromatographic separations such as normal- and reversed-phase HPLC. Normal phase HPLC separates lipids based on their polar head groups while reversed-phase HPLC separates lipids based on their hydrophobicity correlating to the length of their hydrophobic tails. In short, the shorter the lipid tail, the quicker the elution and vice versa. Coupling liquid chromatography to mass spectrometry reduces the complexity of the lipid mixture being injected into the mass spectrometer for analysis. This front end chromatographic separation is advantageous as it limits high abundant lipid species to a relatively narrow chromatographic window, therefore allowing low-abundance lipid species to be identified with less interference and with higher confidence. ${ }^{8}$

Mass spectrometric lipid studies using direct infusion have a simpler experimental setup, removing the need for sample processing steps and allows for high throughput analysis. However, certain classes of lipid species are preferentially ionized under different experimental conditions. This inevitably leads to ionization suppression of other lipid classes, in particular those that have a lower proton affinity, since there is a limit on the amount of total ionization that can occur at any one instant through ESI in the positive ion mode. ${ }^{8}$ 
Structural elucidation of lipids is one of the challenges in the field of lipidomics. ${ }^{8}$ Mass spectrometry-based approaches for structural elucidation of glycerophospholipids in the positive ion mode are described. Unlike proteomic studies, where CID is often used to provide sequence information, CID on lipids is used to enable identification of specific lipid classes via the characteristic fragments associated with a polar head group or a fatty acyl moiety. ${ }^{8}$ Glycerophospholipids such as PC, PE and PS, and sphingolipids such as SM have been analyzed by positive ESI-MS/MS. ${ }^{55,56} \mathrm{CID}$ of protonated PC and SM lipids yields the diagnostic fragment for the phosphocholine head group at $\mathrm{m} / \mathrm{z} 184 .{ }^{57,58}$ This characteristic $\mathrm{m} / \mathrm{z} 184$ fragment is often used in the precursor ion scanning mode to highlight all the phosphocholine-containing lipids during an analysis. CID on a protonated $\mathrm{PE}\left([\mathrm{M}+\mathrm{H}]^{+}\right)$typically yields a peak of the parent ion minus $141 \mathrm{Da}\left([\mathrm{M}+\mathrm{H}-141]^{+}\right)$. This characteristic loss of $141 \mathrm{Da}$ is commonly used to study PE using the neutral loss scanning mode. Similarly, an $\mathrm{m} / \mathrm{z}$ value of $[\mathrm{M}+\mathrm{H}-185]^{+}$in $\mathrm{MS}^{2}$ analysis can be used to confirm the presence of protonated PS, the neutral loss of the phosphoserine head group during CID. However, these neutral loss studies are highly dependent on the analytes' ability to be protonated, which may limit the sensitivity of the analysis. Figure 1.25 highlights the common fragments that correspond to the aforementioned masses. 


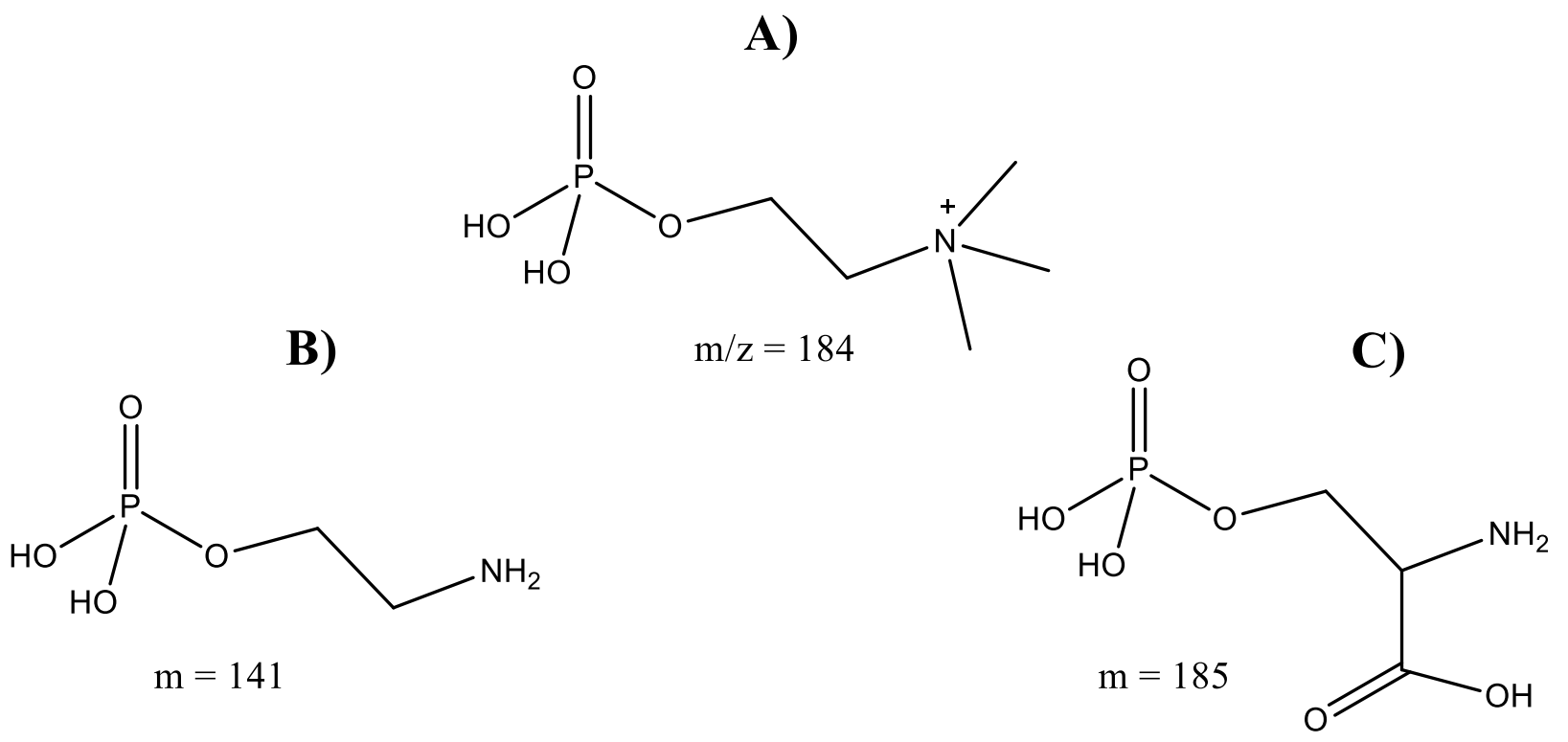

Figure 1.25: A) The precursor fragment for PC and SM; B) the neutral loss fragment for PE; C) the neutral loss fragment for PS

\subsection{Diazomethane}

Diazomethane can be easily synthesized in two separate one step reactions (Figure 1.26). It is a yellow gas that is most commonly dissolved in a solvent such as diethyl ether. ${ }^{59}$ This gas has several dangers associated with it, including its high toxicity, potential carcinogenic properties and its ability to explode unaccountably. ${ }^{60,61}$ Therefore, extra precautions are required when working with this compound such as a well-ventilated fume hood, using special glassware free of sharp edges or ground joints and working behind a heavy blast shield. Despite these preparative problems, diazomethane has several advantages such as mild reaction conditions and facile reaction monitoring through the disappearance of its yellow 
colour and effervescence of nitrogen gas. $^{62}$ This last step is both kinetically and thermodynamically favourable which helps to drive the reaction to completion. Chemistry involving diazomethane is most commonly performed in solution as an instantaneous methylating agent, particularly to methyl esterify carboxylic acids (Figure 1.27). In solution, work has been previously performed on both lipids and peptides and a brief description will be presented here.
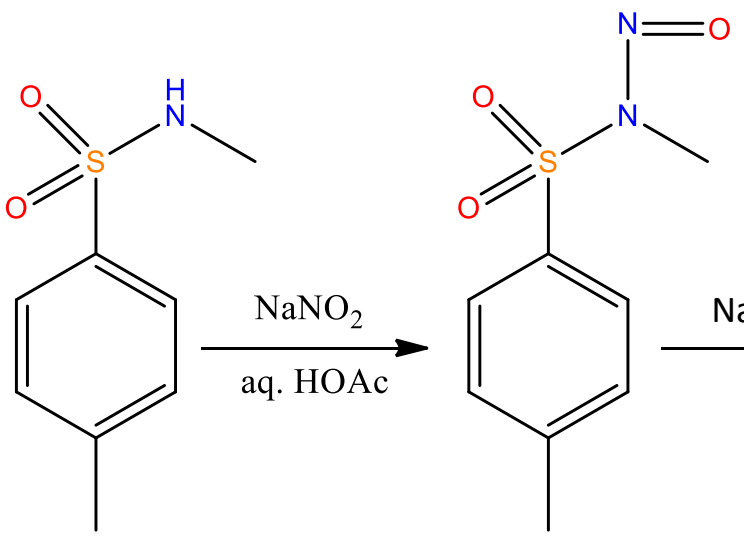

$\mathrm{NaOH}$

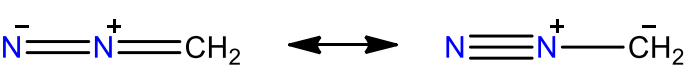

Figure 1.26: Two step reaction to synthesize diazomethane

Fatty acyls are commonly studied using gas chromatography (GC). Of all the lipid classes, diazomethane has been most extensively used on the fatty acyls (Figure $1.7 \mathrm{~A}$ ) to methyl esterify the carboxylic acid prior to GC separation and analysis. ${ }^{63,64,65}$ Evidence of extensive phospholipid fatty acid methylation has been shown to occur during the assumed selective methylation of plasma free fatty acids by diazomethane; ${ }^{66}$ however, phospholipid fatty acid methylation was not shown to be complete. Diazomethane in solution work has also been reported on dipalmitoyl glycerophosphoethanolamine. ${ }^{67}$ This work effectively trimethylated a lipid primary amine to produce a choline moiety, a desirable effect for mass spectrometric 
analysis as the choline moiety possesses a fixed positive charge. However, no methylation was observed on the phosphate moiety. Similarly, work conducted in solution using ethereal diazomethane has also been reported on peptides to produce some amino acid artifacts containing $\mathrm{N}$-methyl, and even $\mathrm{N}, \mathrm{N}$-dimethyl amino acids. ${ }^{68}$ However, this was only produced in small quantities and to no significant advantage. ${ }^{68}$ Trimethylation on the N-terminus of small di- and tri- peptides was achieved using gaseous diazomethane ${ }^{69}$ though success on larger peptides has yet to be reported.

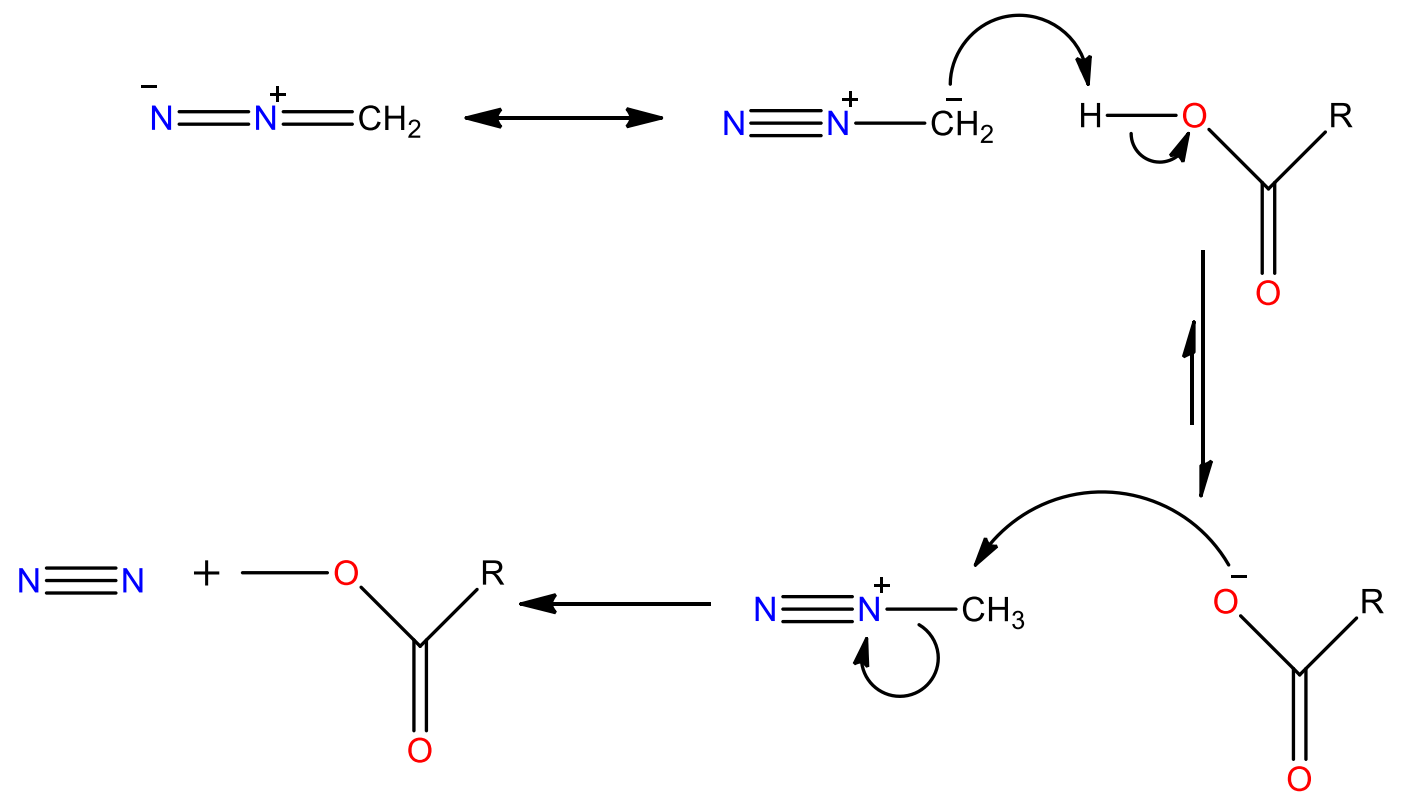

Figure 1.27: Diazomethane methyl esterifying a carboxylic acid

In an attempt to produce the desired, trimethyl $\mathrm{N}$-termini of peptides, other derivatization methods have been explored such as methylation with methyl iodide. This chemistry has been shown to be successful with trimethylating the $\mathrm{N}$-terminus on several occasions ${ }^{70,71,72}$ however, methyl iodide is also hazardous and trimethylation using methyl 
iodide requires specialized equipment, including in vacuo systems to operate for an extensive period of time for derivatization to occur. ${ }^{70,71,72}$ Another derivatization reagent used for methylation is trimethylsilyldiazomethane (TMSD). Researchers often prefer TMSD over diazomethane for methylation of carboxylic acids as it is far more stable and therefore far less likely to explode. However, its slower reaction kinetics may prevent full methylation on the desired moieties, ${ }^{73}$ and trimethylation of primary amines has not been as successful using TMSD. $^{74}$

The focus of this thesis is on the application of diazomethane in a new technique that was developed to assist in the analysis of both peptides and lipids. The technique is called Trimethylation Enhancement using Diazomethane (TrEnDi). TrEnDi differs from other diazomethane studies in that it maximizes the methylation capability of diazomethane by producing the necessary conditions for the methylation of both peptides and lipids. TrEnDi effectively methylates both peptides and lipids in specific locations to produce fixed positive charges, a highly desirable feature for mass spectrometric analysis. Furthermore, TrEnDi modified peptides and lipids produce characteristic fragments upon collisional activation. This translates into the ability to conduct predictable and highly sensitive, precise and accurate label-free quantitative analyses. The specific conditions, results, advantages and future applications to using TrEnDi are discussed in detail in subsequent chapters. 


\subsection{Materials and methods}

Below are the general materials and methods used throughout various experiments performed in this thesis. More concise methods can be found in individual chapters.

$N$-methyl-p-toluenesulfonamide, ammonium acetate, formic acid, tetrafluoroboric acid, $\mathrm{H}_{2}{ }^{18} \mathrm{O}$ and sodium hydride were purchased from Sigma-Aldrich (St. Louis, MO, USA), ethanol was purchased from Commercial Alcohols INC., (Brampton, ON, Canada), trypsin and formamide were purchased from Promega Corp. (Madison, WI, USA), potassium hydroxide, sodium hydroxide, methanol, isopropanol and ether were purchased from Caledon Laboratories LTD (Georgetown, ON, Canada), sodium nitrite was obtained from BDH Chemicals LTD (Poole, England), phosphoric acid was obtained from BDH Inc. (Toronto, ON, Canada), glacial acetic acid was obtained from Anachemia Canada Inc. (Montreal, QC, Canada), potassium silicate 1624 was obtained from National Silicates (Toronto, ON, Canada), acetonitrile was purchased from Fischer Scientific (Fair Lawn, NJ, USA), Zip-tips were obtained from Millipore Corp., (Billercia, MA), ammonium bicarbonate was purchased from MP Biomedicals, LLC (Solon, OH, USA), Magic C18 was obtained from Michrom Bioresources, C4 resin was obtained from $\mathrm{Dr}$. Maisch $\mathrm{GmbH}$, and Omix-tips were obtained from Varian (Lake Forest, CA, USA). FLEEVK, FLEENK, FLEEVR, FLEEYK, FLEEHK, FLEEpSK and FLEEpYK were synthesized by and obtained from CanPeptide Inc. (Pointe-Claire, QC, Canada). All proteins were purchased from Sigma Aldrich (St. Louis, MO, USA). PS (18:0,18:2), PE(16:0,18:1), PC(18:1,14:0), PC(16:0,18:1) and SM(d18:1/16:0) were synthesized by and obtained from Avanti 
Polar Lipids Inc. (Alabaster, Alabama, USA). Fused silica was obtained from Molex (Lisle, IL, USA) and PolySULFOETHYL A Bulk Resin, $12 \mu \mathrm{m} 300 \AA \mathrm{SCX}$ resin was obtained from The Nest Group, Inc.

Special instruments and glassware used include: a high pressure vessel (HPV) (built in house), a Dionex 3000 Ultimate HPLC system (Thermo Scientific, Waltham, MA, USA), an AB Sciex 4000 QTRAP hybrid triple quadrupole linear ion trap mass spectrometer (AB Sciex, Framingham, MA, USA), an AB Sciex QStar XL mass spectrometer ( $A B$ Sciex, Framingham, MA, USA), Proxeon nanoES capillary tips (Odense, Denmark), a Sigma-Aldrich Mini Diazald Diazomethane generator with Clear-Seal joints (St. Louis, MO, USA), Harvard apparatus 11 plus syringe pump (Harvard Apparatus, Holliston, MA, USA), $2 \mathrm{~mL}$ clear screw vials (Canadian Life Sciences, Peterborough, On, Canada) and a TurboV ${ }^{\mathrm{TM}}$ ion source (AB Sciex, Framingham, MA, USA)

\section{$2.1 N$-methyl- $N$-nitroso-p-toluenesulfonamide (Diazald) production}

$N$-methyl- $N$-nitroso- $p$-toluenesulfonamide was synthesized from $N$-methyl- $p$ -

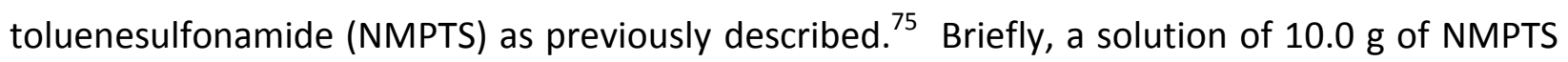
and $22 \mathrm{~mL}$ of water was heated at $85^{\circ} \mathrm{C}$ until the NMPTS was in a molten form. The hot mixture was then quickly transferred to a solution containing $50 \mathrm{~mL}$ of acetic acid. The NMPTS mixture was allowed to cool in an ice bath for 20 minutes and a solution containing $4.24 \mathrm{~g}$ of sodium nitrite $\left(\mathrm{NaNO}_{2}\right)$ in $9 \mathrm{~mL}$ of water was then added drop-wise over 30 minutes with constant 
stirring and maintaining the internal temperature below $10^{\circ} \mathrm{C}$. After addition of the $\mathrm{NaNO}_{2}$ solution was complete, the reaction was allowed to stir for 15 minutes. The product was then rinsed with approximately $1.5 \mathrm{~L}$ of distilled water, vacuumed filtered and allowed to air dry.

\subsection{Diazomethane production}

All reactions involving the preparation of diazomethane should be carried out in an efficient chemical fume hood and behind a safety blast shield because of the toxic and explosive nature of diazomethane. The production of diazomethane was performed using a SigmaAldrich Mini Diazald Diazomethane generator with Clear-Seal joints. The production of diazomethane with this apparatus has been described elsewhere, ${ }^{76}$ and only a brief outline is reported here. $10 \mathrm{~mL}$ of $95 \%$ ethanol was added to a solution containing $5 \mathrm{~g}$ of potassium hydroxide and $8 \mathrm{~mL}$ of water in the Mini Diazald reaction vessel, which was stirred for the duration of the reaction. A separatory funnel with clear-sealed joints containing a solution with $5 \mathrm{~g}(23 \mathrm{mmol})$ of Diazald in $45 \mathrm{~mL}$ of ether was placed over the reaction vessel. The cold finger was filled with dry ice and isopropyl alcohol, and the vessel was heated to approximately $75^{\circ} \mathrm{C}$ using an oil bath. The rate of diazomethane/ether distillation was occurring at approximately the same rate as the drop-wise addition of the Diazald in ether solution. The diazomethane in ether solution had a concentration of approximately $0.23 \mathrm{M}$ as determined by titration with glacial acetic acid. 


\subsection{General on-column derivatization procedure}

An $18 \mathrm{~cm}$ long piece of fused silica was cut and fritted using a 1:3 solution of formamide and potassium silicate 1624 . The frit was cut to an appropriate length (approximately 1-2 mm) followed by the packing of the column with PolySULFOETHYL A Bulk Resin, $12 \mu \mathrm{m} 300 \AA \mathrm{A}$ SCX resin obtained from The Nest Group, Inc. The resin was washed and conditioned prior to use. The SCX solution was placed in a high pressure vessel (HPV), where the pressure was allowed to increase between 100 and 400 psi as required. The column was packed with the SCX resin to various lengths averaging between 10 to $12 \mathrm{~cm}$ and the column was dried using nitrogen gas. $75 \mu \mathrm{L}$ of a $10 \mathrm{mmol}$ potassium phosphate buffer (KPB) solution at $\mathrm{pH} 3$ was inserted in the HPV and was allowed to flow through the column at approximately 400 PSI. The column was allowed to dry using nitrogen gas. A solution containing between 7 and $15 \mu \mathrm{g}$ of analyte was mixed with a $50 \mathrm{mM} \mathrm{H} \mathrm{PO}_{4}$ solution $(\mathrm{pH} 2.1)$ in a 1:9 ratio before it was packed onto the $\mathrm{SCX}$ column at approximately $300 \mathrm{PSI}$. Bound analytes were allowed to dry using nitrogen gas. A required amount of diazomethane (between 150 to $500 \mu \mathrm{L}$ depending on individual experiments) was added to the HPV and was allowed to flow through the column. The effervescence of nitrogen gas from the end of the column was observed. An elution solution was then passed through the column to elute the analytes from the column. Some elution solutions were chosen for different effects. Once the elution solution was collected, the sample was either analyzed directly via mass spectrometry or subjected to a desalting cleanup step prior to analysis. 


\subsection{General desalting method}

Desalting was accomplished using either Zip-tips or Omix-tips packed with C18 reversed phase stationary phase and an appropriate pipette. Each Zip-tip and Omix-tip had a binding capacity of approximately $2 \mu \mathrm{g}$ and $80 \mu \mathrm{g}$ respectively. Two solutions were prepared to desalt: solution $A$ was a solution comprised of $70 \%$ aqueous acetonitrile (ACN) and $1 \%$ formic acid; solution B was comprised of Milli-Q water and $1 \%$ formic acid. The tip was washed with solution A three times, followed by wetting with solution B three times before binding the analyte of choice to the C18. Once analyte was bound, it was washed with solution B five times followed by three elutions with solution $A$.

\subsection{General in solution derivatization}

Analyte of choice was placed in a small clear auto-sampler vial that was previously flushed with nitrogen gas, followed by dilution with approximately $10 \mu \mathrm{L}$ of ethanol depending on starting analyte. Sample was subjected to various amounts (in $\mu \mathrm{L}$ ) to either a 14:1 or a 9:1 solution of ether and tetrafluoroboric acid $\left(\mathrm{HBF}_{4}\right)$. It is important that the $\mathrm{HBF}_{4}$ solution be fresh otherwise it will interfere with the derivatization. Diazomethane was added until solution remained yellow (approximately 250 to $700 \mu \mathrm{L}$ ) followed by immediate drying under nitrogen gas. Once completely dried, sample was resuspended in ethanol and ready for analysis. 


\subsection{Tryptic digestion of proteins}

Proteins of choice were dissolved in $50 \mathrm{mM}$ ammonium bicarbonate and were denatured by heating at $37^{\circ} \mathrm{C}$ overnight. The proteins were reduced using dithiothreitol (DTT) at a final concentration of $5 \mathrm{mM}$ and were heated at $56^{\circ} \mathrm{C}$ for 1 hour. The reduced proteins were allowed to cool to room temperature. lodoacetamide was added until the solution consisted of $10 \mathrm{mM}$ concentration followed by incubation in the dark for 1 hour. The trypsin was re-constituted into a $50 \mathrm{mM}$ ammonium bicarbonate solution and was added to each protein in a 1:20 (w/w; protease:protein) ratio. The proteins were allowed to incubate over night at $37^{\circ} \mathrm{C}$.

\subsection{Generation of $\mathrm{Na}^{18} \mathrm{OH}$}

$24 \mathrm{mg}$ of $\mathrm{NaH}$ was placed in $1 \mathrm{~mL}$ of $\mathrm{H}_{2}{ }^{18} \mathrm{O}$ to produce a $1 \mathrm{~mL}$ solution of $\sim 1 \mathrm{M} \mathrm{Na}{ }^{18} \mathrm{OH}$.

\subsection{General direct analysis via mass spectrometry}

To obtain accurate readings, the MS was calibrated using a cesium iodide solution before each use. 1.5 to $5 \mu \mathrm{L}$ of analyte solution was loaded into a Proxeon nanoES capillary tip and affixed to the Qstar-XL AB Sciex mass spectrometer. Unless otherwise stated, the analyte 
solution was comprised of approximately $0.050 \mu \mathrm{mol}$ of analyte dissolved in $1 \mathrm{~mL}$ water containing $0.1 \%$ formic acid. The tip of the nanospray emitter was gently bent against the charged curtain plate to enable the desired spray of solution into the MS. The settings most often used in Analyst QS software were as follows: Ion Source Gas 1 set to 1, Ion Spray Voltage set to 1000 , and TOF Mass Min and Max values set from 400 to 500 and 700 to 900 to capture the mass range of the unmodified and modified analytes. Gas source, Ion Spray Voltage and TOF mass values were altered to suit each individual experiment.

\subsection{LC-MS/MS Analysis}

All LC-MS/MS analysis for both peptides and lipids were separated using a Dionex 3000 Ultimate HPLC system. Analysis was conducted using various modes on the AB Sciex 4000 QTRAP hybrid triple quadrupole linear ion trap mass spectrometer equipped with an online ESI source (lipids) or nanoESI source (peptides) coupled to the HPLC eluate.

\subsubsection{LC-MS/MS Analysis of peptides and proteins}

The reversed-phase chromatography pre-columns and analytical columns were packed with Magic C18 resin via HPV. A piece of fused silica with an inner diameter of $200 \mu \mathrm{m}$ was cut and fritted using a 1:3 solution of formamide and potassium silicate 1624 . The frit was cut to 
an appropriate length (approximately 1-2 $\mathrm{mm}$ ) followed by the packing of the column with Magic C18 resin to a length of approximately $5 \mathrm{~cm}$. Approximately $4 \mathrm{~cm}$ of Magic C18 resin was packed inside a PicoFrit ${ }^{\mathrm{TM}}$ Emitter with a starting inner diameter of $75 \mu \mathrm{m}$ and a $15 \mu \mathrm{m}$ opening at the tip. HPLC runs varied from 12 minutes to 30 minutes with a gradient of $0.1 \%$ formic acid/water and $0.1 \%$ formic acid/acetonitrile and with various flow rates to optimize peptide elution. An example of a common gradient for peptides and proteins is shown in Figure 2.1. Following separation by reversed-phase chromatography, the peptides were electrosprayed using a nanoESI voltage of $3500 \mathrm{~V}$, a declustering potential of $25 \mathrm{~V}$ and a collision energy of 33 eV. Analysis was conducted using both IDA MS ${ }^{2}$ and MRM modes on the AB Sciex 4000 QTRAP. In the IDA MS ${ }^{2}$ mode, peptides were identified using IDA Explorer to search for $\mathrm{MS}^{2}$ spectra on the expected mass. In the MRM mode, peptide identification was accomplished by monitoring two ion transitions.

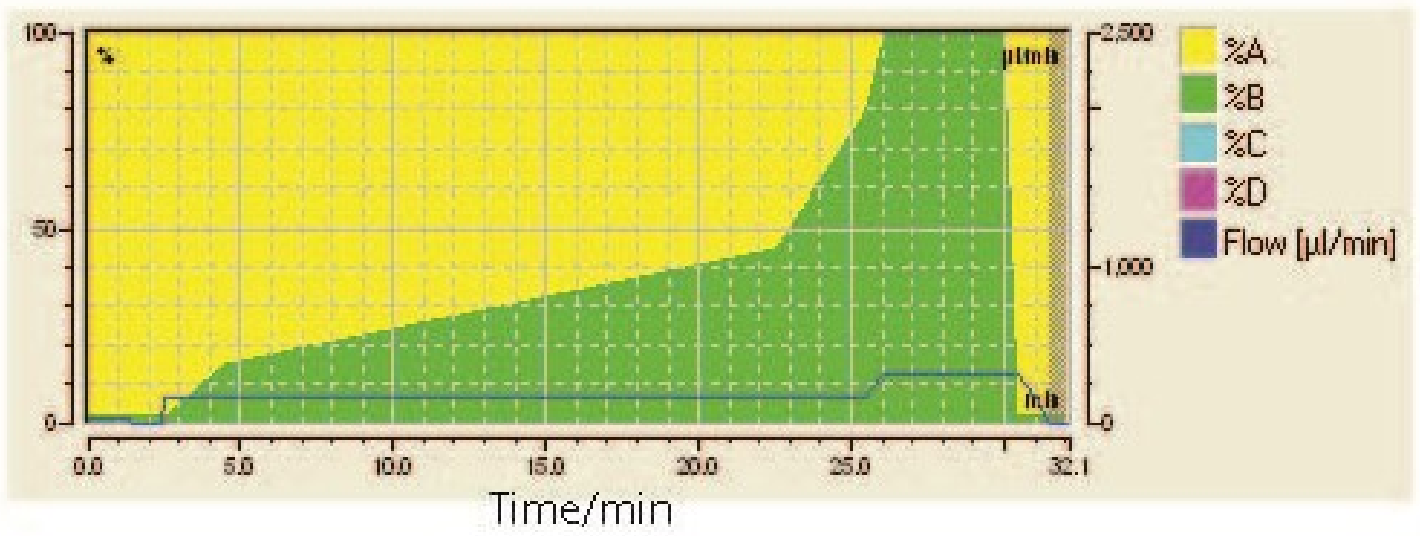

Figure 2.1: A gradient used for peptide elution from HPLC. $A=\mathrm{H}_{2} \mathrm{O}$ and $0.1 \%$ formic acid. $\mathrm{B}=\mathrm{ACN}$ and $0.1 \%$ formic acid. 


\subsubsection{MS/MS Analysis of lipids}

A piece of fused silica with an inner diameter of $200 \mu \mathrm{m}$ was cut and fritted using a 1:3 solution of formamide and potassium silicate 1624 . The frit was cut to an appropriate length (approximately 1-2 $\mathrm{mm}$ ) followed by the packing of the column with $\mathrm{C} 4$ resin to a length of approximately $5 \mathrm{~cm}$. A TurboV ${ }^{\mathrm{TM}}$ ion source with a $100 \mu \mathrm{m}$ inner diameter electrode was used. HPLC runs were 50 minutes in duration with a gradient of $30 \%$ methanol/70\% water/10 mM ammonium acetate and $100 \%$ isopropanol/10 $\mathrm{mM}$ ammonium acetate with various flow rates to optimize lipid elution. The gradient most often used for lipid separation on HPLC is shown in Figure 2.2. Following separation by reversed-phase chromatography, the lipids were electrosprayed using an ESI voltage of $5000 \mathrm{~V}$, a declustering potential of $80 \mathrm{~V}$ and a collision energy of $40 \mathrm{eV}$. Analysis was conducted using MS and precursor ion scanning mode on the $A B$ Sciex 4000 QTRAP. In the precursor ion scanning mode, lipids were identified using the expected mass of the polar head group post TrEnDi.

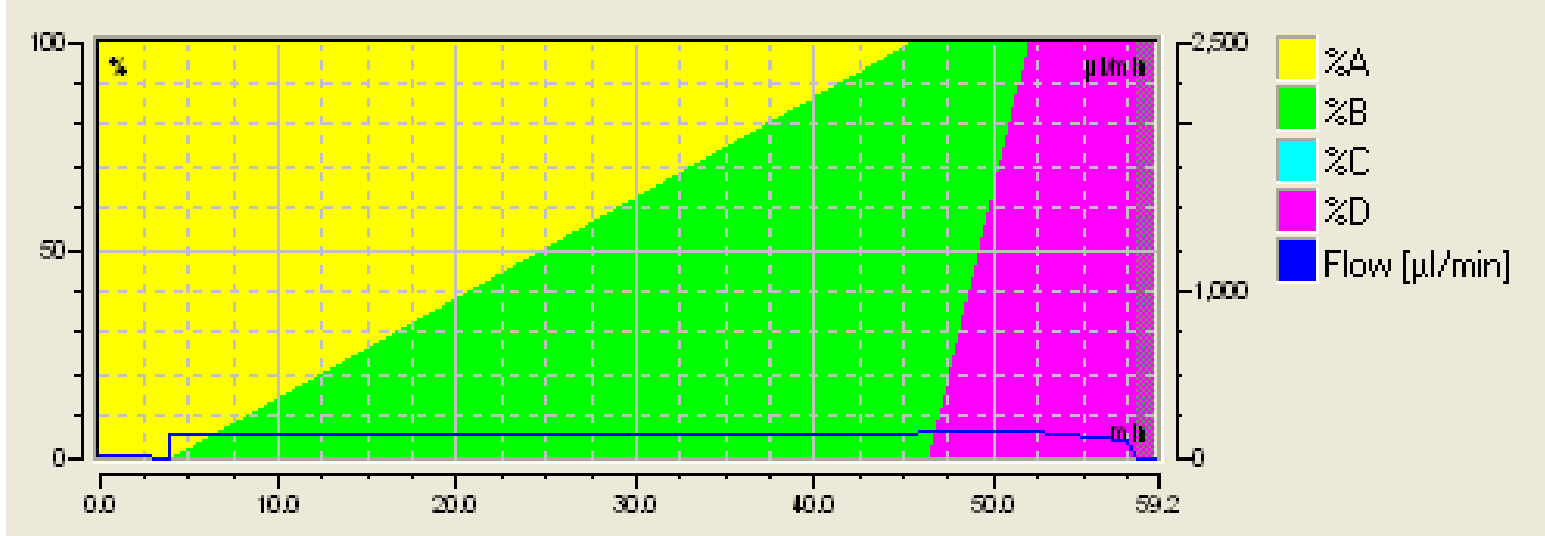

Figure 2.2: Gradient used for lipid elution. $A=30 \%$ methanol $/ 70 \%$ water $/ 10 \mathrm{mM}$ ammonium acetate; $B=100 \%$ isopropanol $/ 10 \mathrm{mM}$ ammonium acetate; $D=$ Hexane 


\subsection{MS/MS analysis of lipid via direct infusion}

A solution of ethanol containing lipids was directly infused into the 4000 Qtrap via electrospray emitter using a Harvard apparatus 11 plus syringe pump. Lipids were injected at a rate of $0.5 \mu \mathrm{L} / \mathrm{min}$ using an ESI voltage of $5000 \mathrm{~V}$, a declustering potential of $40 \mathrm{~V}$ and a collision energy of 40 and/or $60 \mathrm{eV}$. Analysis was conducted using EMS and tandem experiments were conducted using both the precursor ion scanning and neutral loss scanning modes. 


\subsection{TrEnDi on peptides and proteins}

New microfluidic devices have recently emerged to process proteomic samples and allow digestion of very small samples one at a time, ${ }^{77}$ within an array $^{78}$ or on a rapid time scale. $^{79}$ Microfluidic platforms have been used to enrich protein post-translational modifications $(\mathrm{PTMs})^{80,81}$ as well as conduct specific chemical reactions on peptides. ${ }^{82}$ Microfluidic platforms alter the kinetics of a chemical reaction compared to the solution phase and thereby offer unique chemical reaction opportunities. ${ }^{77}$ Furthermore, microfluidic devices allow convenient and precise control over sample handling, leading to the introduction of less error and thereby increasing the precision of proteomic analyses.

Peptides have unique, sequence-dependent proton affinities, meaning that the relative intensities of different peptides in a single mass spectrum do not necessarily correlate to their concentrations; ${ }^{83}$ as a result, different quantitation strategies have been developed. Many quantitation methods involve the incorporation of stable isotopes such as ${ }^{2} \mathrm{H},{ }^{13} \mathrm{C},{ }^{15} \mathrm{~N}$ or ${ }^{18} \mathrm{O}$ so that peptides of the same sequence from different samples may be simultaneously analyzed and yet have distinctive masses. Stable isotopes may be introduced chemically, ${ }^{84}$ enzymatically ${ }^{85}$ or metabolically, ${ }^{86}$ permitting quantitation via comparing the relative intensities of each isotopically labeled peptide in a mass spectrum; each method possesses advantages and limitations. A variety of methods to chemically label peptides with stable isotopes have appeared over the past decade ${ }^{87}$ including those that contain affinity tags, such as ICAT, ${ }^{88}$ as well as those that label peptides with isobaric tags that permit quantitation at the $\mathrm{MS}^{2}$ level, 
such as iTRAQ $^{89}$ and $\mathrm{TMT}^{90}$ Stable isotope introduction has also been accomplished through the enzymatic hydrolysis of peptide bonds in either ${ }^{16} \mathrm{O}$-labeled or ${ }^{18} \mathrm{O}$-labeled water resulting in a mass shift due to the incorporation of an isotopically distinct carboxy-terminal oxygen. ${ }^{85}$ Stable isotope labeling by amino acids in cell culture (SILAC) ${ }^{86}$ incorporates stable isotopes within proteins at a metabolic level as cells are grown in isotopically enriched media. SILAC offers the advantage of reducing inter-sample indeterminate error introduced through sample handling as different protein samples are mixed immediately following cell culture; however, it can be expensive and is not feasible on tissues or fluids from larger organisms.

As an alternative to isotopic labeling, "label free" strategies have also been developed for protein quantitation. ${ }^{91}$ Label-free quantitation requires the chromatographic separation of an analyte solution coupled with MS analysis; analyte quantities are calculated by integrating the area under the MS intensity curve that is produced as they elute, a technique that has been established for over half a century. ${ }^{91,92,93}$ In the case of peptides, quantitation is achieved through the creation of a standard curve for a given peptide, comparison to an internal standard with a known response factor or relative quantitation is achieved by comparing the signal of the same peptide in consecutive chromatographic separations. If the sample complexity remains relatively constant, label-free strategies have been demonstrated to be particularly effective. This has been further demonstrated by the emergence of multiple reaction monitoring (MRM) (or selected reaction monitoring (SRM)) strategies (achieving quantitation via $\mathrm{MS}^{2}$-based signal intensities for greater selectivity). ${ }^{94}$ One of the disadvantages of label-free quantitation is that variation in sample complexity can affect the ionization efficiency of a given peptide, ${ }^{95,96}$ introducing error in quantitation. ${ }^{97}$ Furthermore, there are 
many peptides that produce little to no MS signal, as previously documented ${ }^{96}$ and hence cannot be measured.

This thesis work has developed a novel strategy that harnesses the benefits of microfluidics and provides a solution for some of the limitations of label-free peptide quantitation. The technology utilizes a column containing strong cation exchange (SCX) chromatographic resin to expose peptides to an ethereal solution of diazomethane. Diazomethane is most commonly used in solution chemistry as an instantaneous methylating agent, specifically with carboxylic acids to yield methyl esters and very few by-products. ${ }^{98,99}$ Earlier studies have shown that aqueous solution-based studies with gaseous diazomethane have produced trimethylated $\mathrm{N}$-termini on di- and tri-peptides, ${ }^{69}$ and ethereal diazomethane produced some amino acid artifacts containing $\mathrm{N}$-methyl, and even $\mathrm{N}, \mathrm{N}$-dimethyl amino acids in small quantities, but to no significant advantage. ${ }^{68}$ In this chapter, we report a fast and costeffective microfluidic strategy that results in the complete trimethylation of primary amines, producing at least one or two fixed positive charges on tryptic peptides that terminate with arginine or lysine residues, respectively. Moreover, previous research on peptides with trimethylated $\mathrm{N}$-termini has demonstrated the preferential formation of $\mathrm{a}_{2}$ fragment ions upon collisional activation. $^{72}$ This translates into the ability to conduct predictable and highly sensitive MRM-based analyses and therefore more robust, sensitive, precise and accurate labelfree quantitative proteomics analyses. 


\subsection{Sample preparation}

All materials used are explained in section 2.0. Each of the FLEEVK, FLEENK, FLEEVR, FLEEYK and FLEEHK peptides were dissolved in water to prepare concentrations ranging from $1.25 \mathrm{mM}$ to $2.53 \mathrm{mM}$. A combination of 7 proteins ("protmix") consisting of $3.0 \times 10^{-9}$ moles of each $\alpha$-casein, bovine serum albumin (BSA), $\beta$-casein, concanavalin A, hemoglobin, myoglobin, and ovalbumin were mixed together and tryptically digested as previously described. ${ }^{100}$ A 0.5 $\mathrm{mg}$ batch of BSA was tryptically digested separately. The digested protmix and the BSA were then resuspended in water containing $0.1 \%$ formic acid to concentrations of $0.40 \mu \mathrm{g} / \mu \mathrm{L}$ and $0.21 \mu \mathrm{g} / \mu \mathrm{L}$, respectively.

\section{2 $N$-methyl- $N$-nitroso- $p$-toluenesulfonamide (Diazald) production}

See section 2.1

\subsection{Diazomethane production}

See section 2.2 


\subsection{On-column chemical derivatization}

A fritted $18 \mathrm{~cm}$ long piece of fused silica (200 $\mu \mathrm{m}$ ID) was packed to $6 \mathrm{~cm}$ length with SCX resin, washed with $75 \mu \mathrm{L}$ of $\mathrm{KPB}$ and dried with $\mathrm{N}_{2}$ gas as previously discussed (section 2.3). 10 $\mu \mathrm{L}$ of a solution containing approximately $1.2 \mu \mathrm{g} / \mu \mathrm{L}$ of FLEEVK was mixed with $90 \mu \mathrm{L}$ of $50 \mathrm{mM}$ $\mathrm{H}_{3} \mathrm{PO}_{4}$ solution and was loaded onto the column. Peptide was derivatized by running $250 \mu \mathrm{L}$ of the ethereal solution containing diazomethane through the column at $200 \mathrm{psi}$ and at room temperature. Initially, peptide elution was performed with $100 \mu \mathrm{L}$ of $200 \mathrm{mM}$ ammonium bicarbonate solution $(A B C)$. Upon optimization, base hydrolysis of the methyl esters and elution of peptides were performed with $100 \mu \mathrm{L}$ of $\mathrm{NaOH}(1 \mathrm{M})$. After $\mathrm{NaOH}$ elution, peptides were desalted as previously discussed (Section 2.4).

\subsection{ESI-MS and MS ${ }^{2}$ for individual peptides}

$2 \mu \mathrm{L}$ of the desalted solution was inserted into a Proxeon nanoelectrospray emitter followed by direct analysis of the methylated peptides using the AB Sciex QStar XL. Spectra were obtained using a nanoESI voltage of $1000 \mathrm{~V}$, declustering and focusing potentials at $60 \mathrm{~V}$ and $120 \mathrm{~V}$, respectively and an $\mathrm{MS}^{2}$ collision energy for $\mathrm{MS}^{2}$ experiments that ranged between 33 and $35 \mathrm{eV}$. 


\subsection{LC-MRM for multiple peptides}

$1.3 \mu \mathrm{L}$ of solution containing approximately $0.05 \mu \mathrm{g} / \mu \mathrm{L}$ of modified FLEEVK, FLEEYK and FLEEVR and $0.03 \mu \mathrm{g} / \mu \mathrm{L}$ of modified FLEENK was spiked into $80 \mu \mathrm{L}$ of water and $0.1 \%$ FA containing $0.40 \mu \mathrm{g} / \mu \mathrm{L}$ of digested protmix. $20 \mu \mathrm{L}$ of this solution was injected and separated using a Dionex 3000 at a flow rate of $150 \mu \mathrm{L} / \mathrm{min}$. Two buffers, $0.1 \%$ formic acid in water (A) and $0.1 \%$ formic acid in $A C N$ (B), were used, with a linear gradient increase of $4.9 \% / \mathrm{min}$ of buffer B from $2 \%$ to $100 \%$ for the elution of target peptides. $100 \%$ buffer B was allowed to flow at a rate of $150 \mu \mathrm{L} / \mathrm{min}$ for 3 minutes followed by a flow rate increase to $300 \mu \mathrm{L} / \mathrm{min}$ for an additional 4.5 minutes. A $10 \mathrm{~cm}$ column was packed with Magic C18 to a length of $5 \mathrm{~cm}$. Peptides were analyzed via both IDA MS ${ }^{2}$ and MRM modes on the AB Sciex 4000 Qtrap. The ion transitions used in MRM mode included the doubly charged parent ion to $a_{2}$ fragment ion for each synthetic peptide for $100 \mathrm{~ms}$ (See Table 3.3). Spectra were obtained using a nanoESI voltage of $3500 \mathrm{~V}$, a declustering potential of $25 \mathrm{~V}$ and a collision energy of $33 \mathrm{eV}$.

\subsection{BSA protein identification and LC-MRM for modified BSA}

HPLC separation and $\mathrm{MS}^{2}$ IDA scans were performed on a $20 \mu \mathrm{L}$ injection of solution containing $5 \mu \mathrm{g}$ of tryptically digested BSA. The identification of BSA was performed using MASCOT (Matrixscience, Boston, MA, USA) with an ion score cut off of zero from which, a $52 \%$ sequence coverage was observed. A randomly selected peptide (AEFVEVTK) from the 
remaining $48 \%$ sequence that was not observed was targeted for trimethylation enhancement using diazomethane. On-column chemical derivatization was performed on $5 \mu \mathrm{g}$ of tryptically digested BSA using $500 \mu \mathrm{L}$ of diazomethane solution as described above. The derivatized protein digest was eluted with $100 \mu \mathrm{L}$ of $\mathrm{NaOH}(1 \mathrm{M})$ and desalted using a C18 OMIX tip. Identification of the target peptide (AEFVEVTK) was accomplished via MRM by monitoring the transition of the doubly charged parent ion to the $a_{2}$ fragment ion ( $\mathrm{m} / \mathrm{z} 503.8 \rightarrow \mathrm{m} / \mathrm{z} 215.1$ ) using the MRM parameters described above.

\subsection{Results and discussion for TrEnDi proteomics}

Diazomethane has been used to methyl esterify carboxylic acids on peptides in solution; ${ }^{98}$ however, relatively little is known about the reactivity of diazomethane with other amino acid functional groups. Previous reports of solution-phase experiments have suggested that protonated peptide $\mathrm{N}$-termini may also methylate when placed in close proximity to diazomethane. ${ }^{69}$ An attractive feature of peptide $\mathrm{N}$-terminal methylation is that a permethylated $\mathrm{N}$-terminus would contain a fixed positive charge via a quaternary ammonium, enhancing ionization efficiency and sensitivity when analyzed by MS. Initial experiments revealed that solution-based diazomethane derivatization resulted in an array of products arising from incomplete methylation, thereby dividing signal intensity between multiple species (data not shown). However, when used in conjunction with the adsorption of substrate onto SCX resin, diazomethane reacts with peptides causing the complete conversion of primary 
amines into trimethylated quaternary ammonium groups. The method is only successful when peptides are protonated with an acidic solution (phosphoric acid), bound to the SCX column, followed by the removal of aqueous solvent prior to treatment with diazomethane, suggesting that analyte solvation in solution-based studies may disrupt the complete conversion. Figure 3.1 illustrates the reaction of diazomethane with primary amines; acidic ammonium ion protons protonate diazomethane, creating an unstable diazonium cation, which carries out an $\mathrm{S}_{\mathrm{N}} 2$ reaction methylating the amine while releasing $\mathrm{N}_{2}$ gas. Critically, diazomethane reacts further with the remaining protons on the ammonium group and thus ultimately produces a fixed positive charge on the peptide via a quaternary ammonium ion.

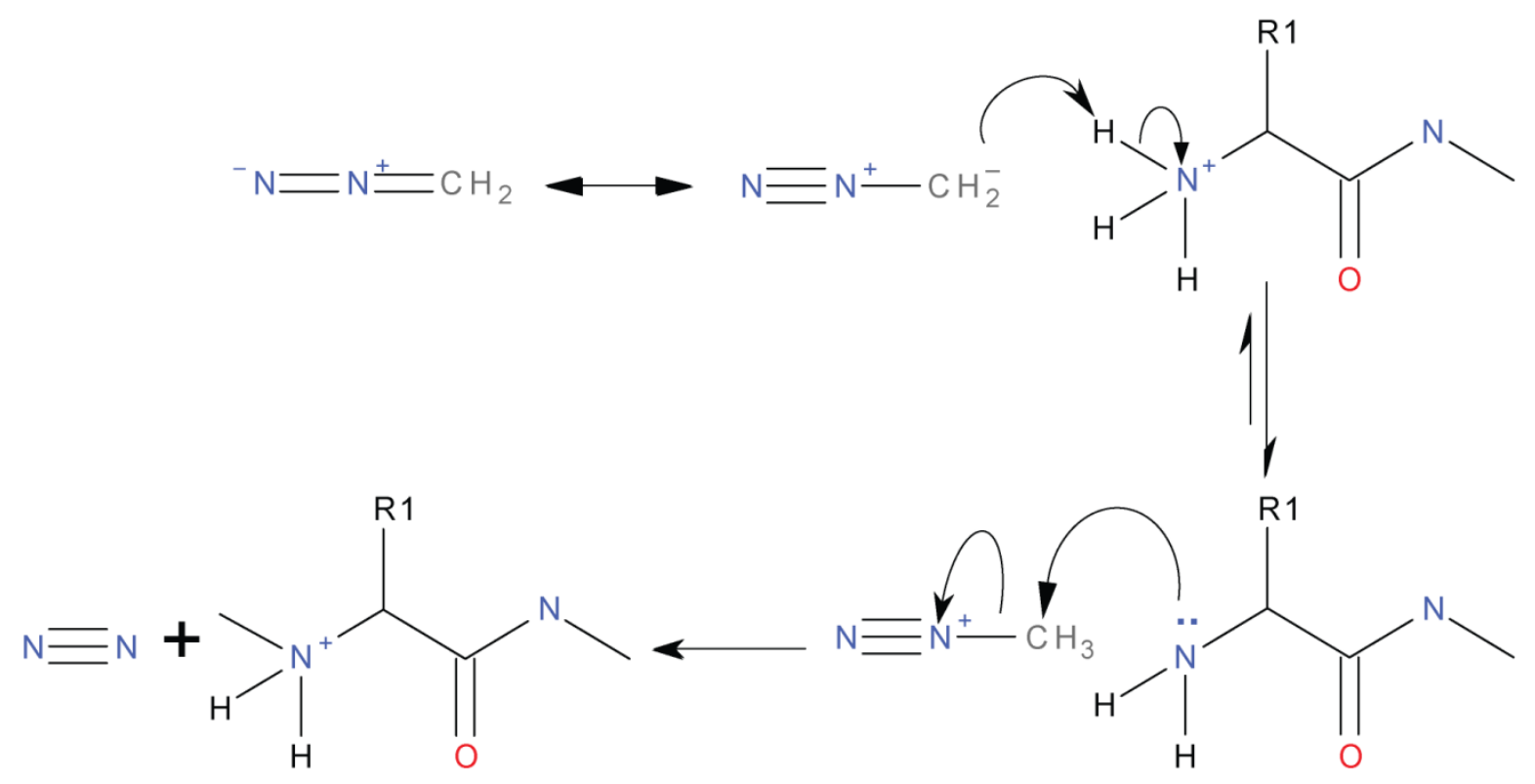

Figure 3.1: Methylation of peptide N-terminus with diazomethane

Initially, a synthetic acidic peptide with the amino acid sequence FLEEVK was chosen to study the chemistry between diazomethane and a peptide within the confines of an SCX microfluidic column. The mass spectrum of this peptide following treatment with 
diazomethane is shown in Figure 3.2. Figure 3.2 a reveals the fully modified $[F L E E V K]^{2+}$ ion at $\mathrm{m} / \mathrm{z}$ 445.76; a total mass shift of +126 Da from the unmodified [FLEEVK] ${ }^{2+}$, indicating that the peptide had been methylated nine times. Tandem mass spectrometry $\left(\mathrm{MS}^{2}\right)$ analysis (Figure 3.2b) reveals the location of these nine newly inserted methyl groups. Trimethylation is observed at the $\mathrm{N}$-terminus as well as on the less acidic primary ammonium ion of the lysine side chain creating two fixed positive charges on the peptide. Methylation was also observed on all three carboxylic acid groups - two glutamic acid side chains as well as the C-terminus. The derivatization chemistry and structure of modified FLEEVK is illustrated in Figure 3.3.

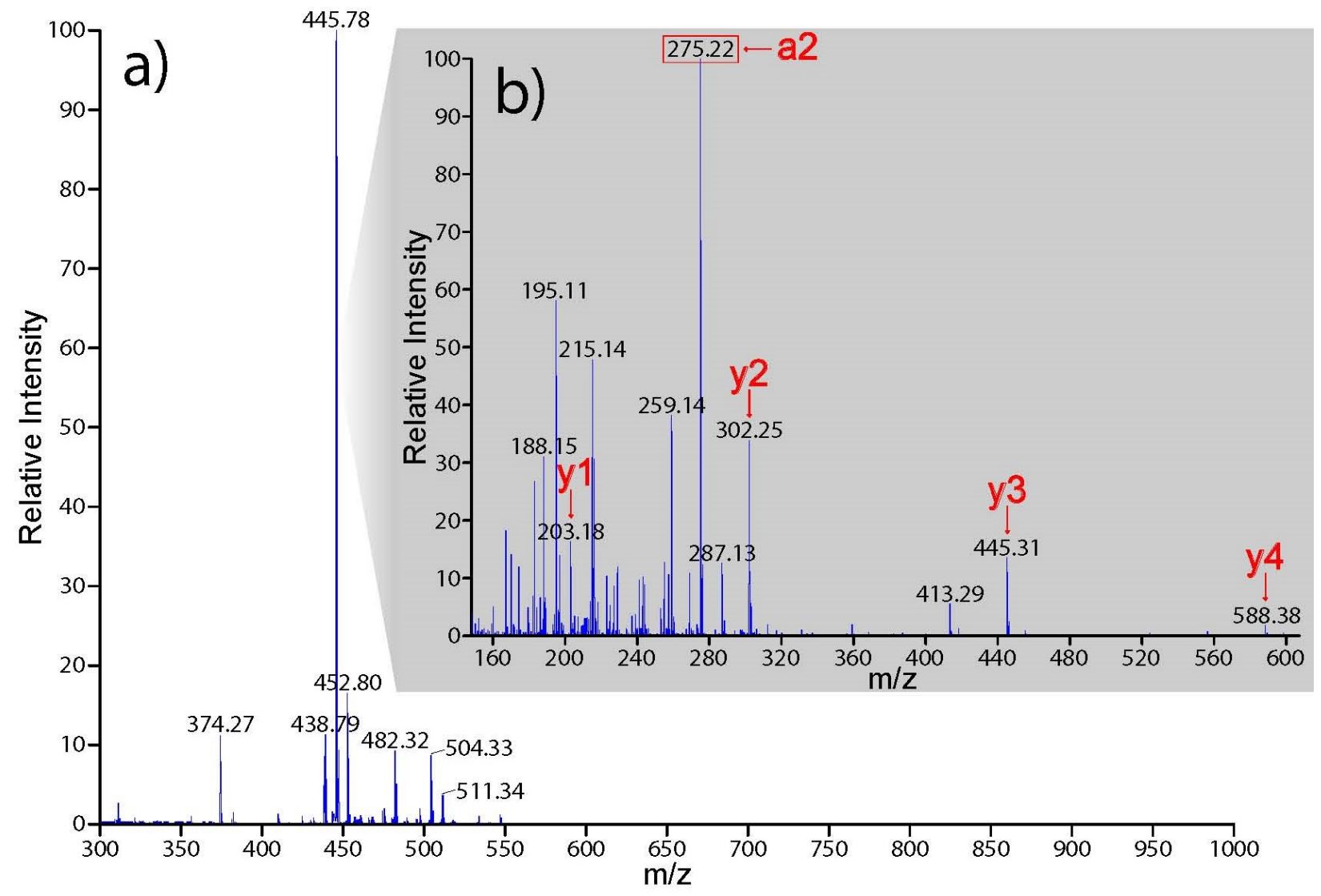

Figure 3.2: a) FLEEVK treated with diazomethane and eluted with ammonium bicarbonate revealing nine added methyl groups ( $\mathrm{m} / \mathrm{z} 445.78)$. b) Fragmentation of this peptide revealed $y$-ions and a predominant $a_{2}$ fragment. 


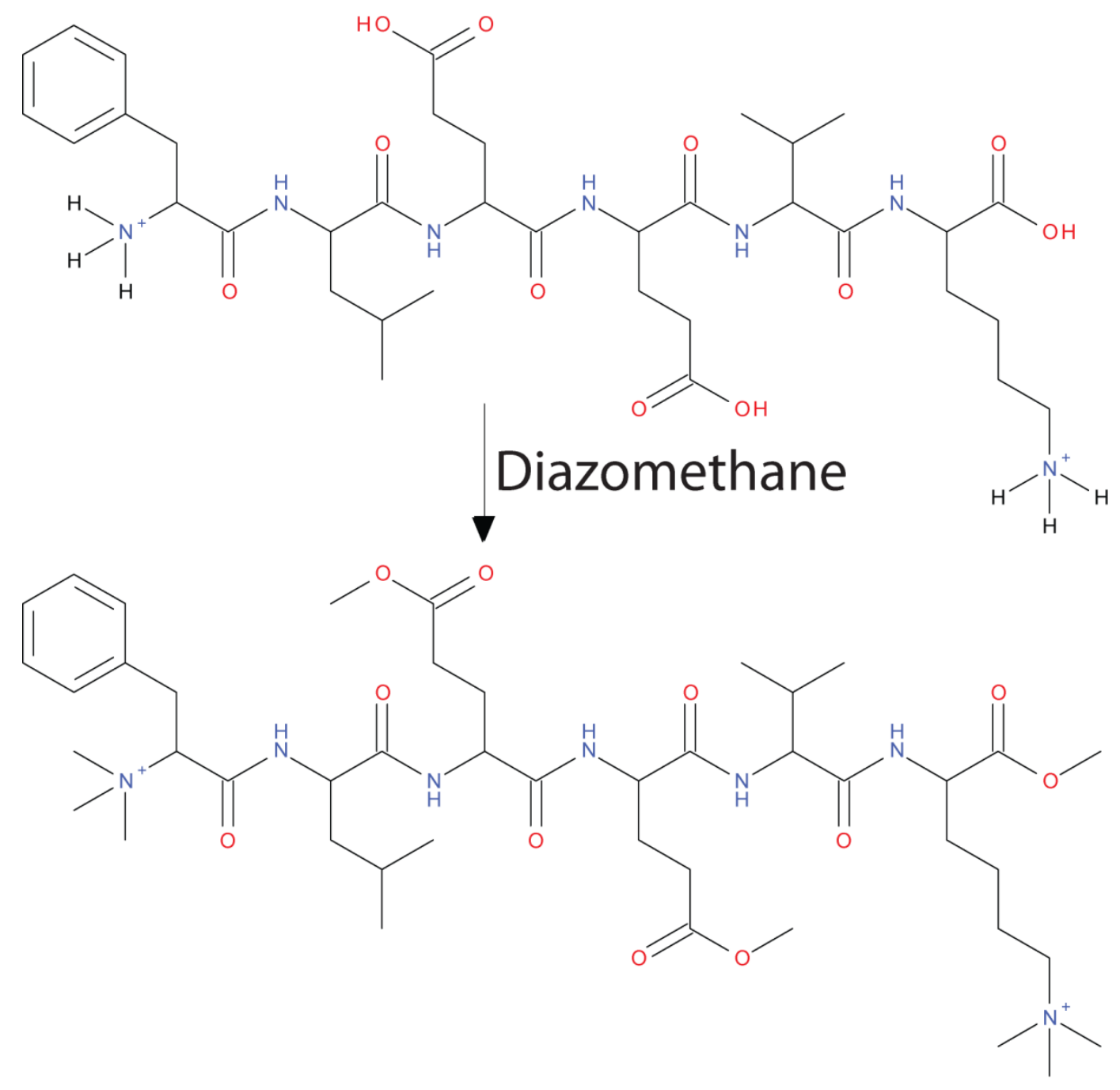

Figure 3.3: Fully modified FLEEVK via treatment with diazomethane

While it was predicted that acidic residues would methyl esterify with diazomethane, our studies indicated that hydrolysis of the newly formed methyl esters occurred over time. It was discovered that this occurred when eluting the peptides from the SCX column with ammonium bicarbonate solution. Further studies also showed that the addition of heat to the overall reaction resulted in primary amide formation at the C-terminus (data not shown), presumably via aminolysis of the methyl ester. Therefore, hydrolysis of the methyl esters was 
performed intentionally by eluting the peptides in $100 \mu \mathrm{L}$ of $1 \mathrm{M} \mathrm{NaOH}$. Figure 3.4 reveals the mass spectrum of diazomethane-modified FLEEVK following base-assisted hydrolysis of the methyl esters where six methylations are observed, confirmed by the dominant molecular ion $[F L E E V K]^{2+}$ at $\mathrm{m} / \mathrm{z} 424.7$ in Figure 3.4a. Again, trimethylation of the $\mathrm{N}$-terminus as well as the primary amine of the lysine side chain was confirmed by $\mathrm{MS}^{2}$ analysis (Figure 3.4b); restoration of the carboxylic acids was also confirmed. The overall reaction of FLEEVK with diazomethane followed by base is illustrated in Figure 3.5 and proved to drive the chemistry to a single, stable product.

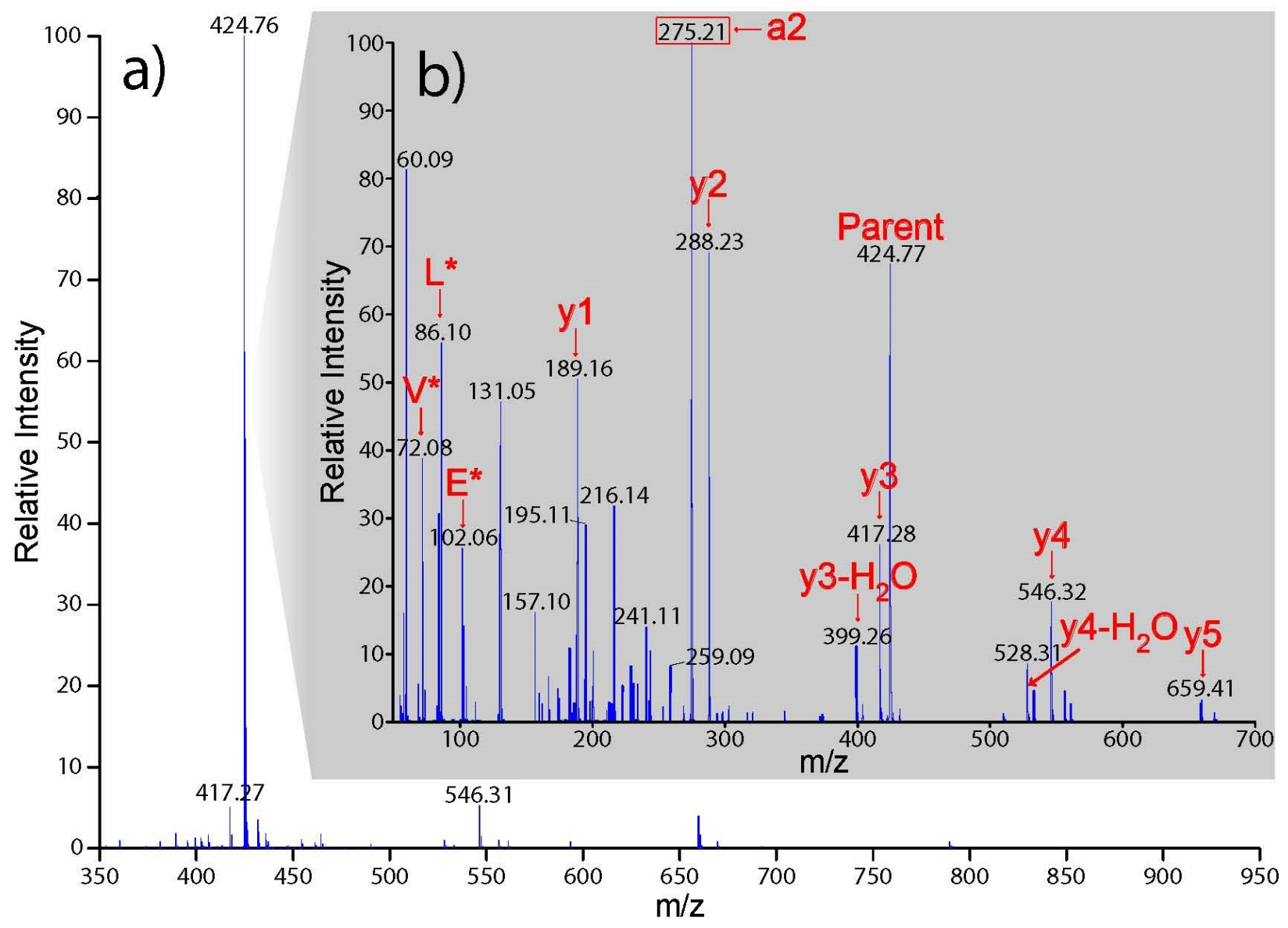

Figure 3.4: a) FLEEVK treated with diazomethane and eluted with sodium hydroxide revealing six added methyl groups ( $\mathrm{m} / \mathrm{z}$ 424.76). b) Fragmentation of this peptide revealed $y$-ions, $y$ ion with the loss of water, immonium ions and a predominant $a_{2}$ fragment. 


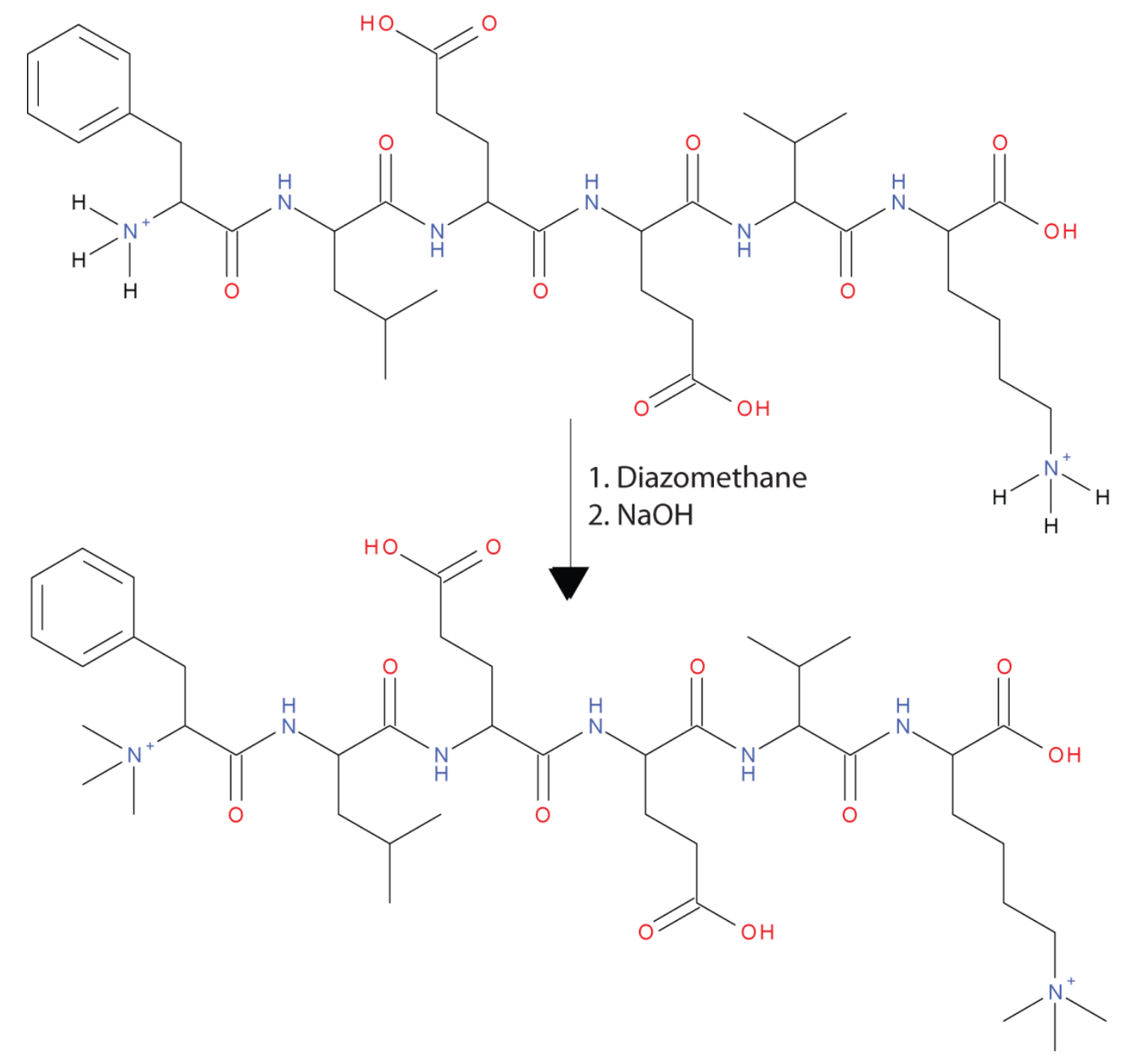

Figure 3.5: Hexamethylated FLEEVK via reaction with diazomethane followed by complete hydrolysis of the methyl esters using $\mathrm{NaOH}$

To further test the reactivity of diazomethane with proton donating amino acids, several other synthetic peptides were purchased and subjected to our methylating strategy. Peptides containing an amide residue $(\mathrm{N})$, a phenol residue $(\mathrm{Y})$, an imidazole residue $(\mathrm{H})$ as well as a guanidinium residue (R) were selected: FLEENK, FLEEYK, FLEEHK and FLEEVR, respectively. 
Results revealed that the histidine imidazole group and the tyrosine phenol group were successfully methylated using our strategy while the arginine guanidine group and the asparagine amide group were not modified; methylation assignments of doubly charged species were confirmed by $\mathrm{MS}^{2}$ analysis (Figure $3.4 \mathrm{~b}$ and Figure 3.7). The MS and $\mathrm{MS}^{2}$ spectra of the triply charged FLEEHK peptide are shown in Figure 3.8. These results indicated that the methylation efficiency of diazomethane is directly proportional to the pKa values of amino acid functional groups. These results indicate that the methylation efficiency of diazomethane is a function of the pKa values of amino acid functional groups - amino acid side chains with pKa values of approximately 11 or less are acidic enough to protonate diazomethane, thereby generating the methyldiazonium ion. Functional groups with pKa values over 11 do not donate a proton within the timeframe of our experiment and are not methylated. Other amino acid residues such as threonine $(T)$ and serine $(S)$ both have R group pKa values of approximately 12 to $13,{ }^{101,102}$ and were therefore not expected to be methylated. This theory was tested on FLEESK and FLEETK. Results indicated that our pKa hypothesis was correct and that the hydroxyl groups from both serine and threonine did not modify to a significant extent within the timeframe of our experiment (Figure 3.6). The results of the treatment of each peptide with diazomethane on an SCX column are listed in Table 3.1. The list of pKa's tested with successful modifications is listed in Table 3.2. 


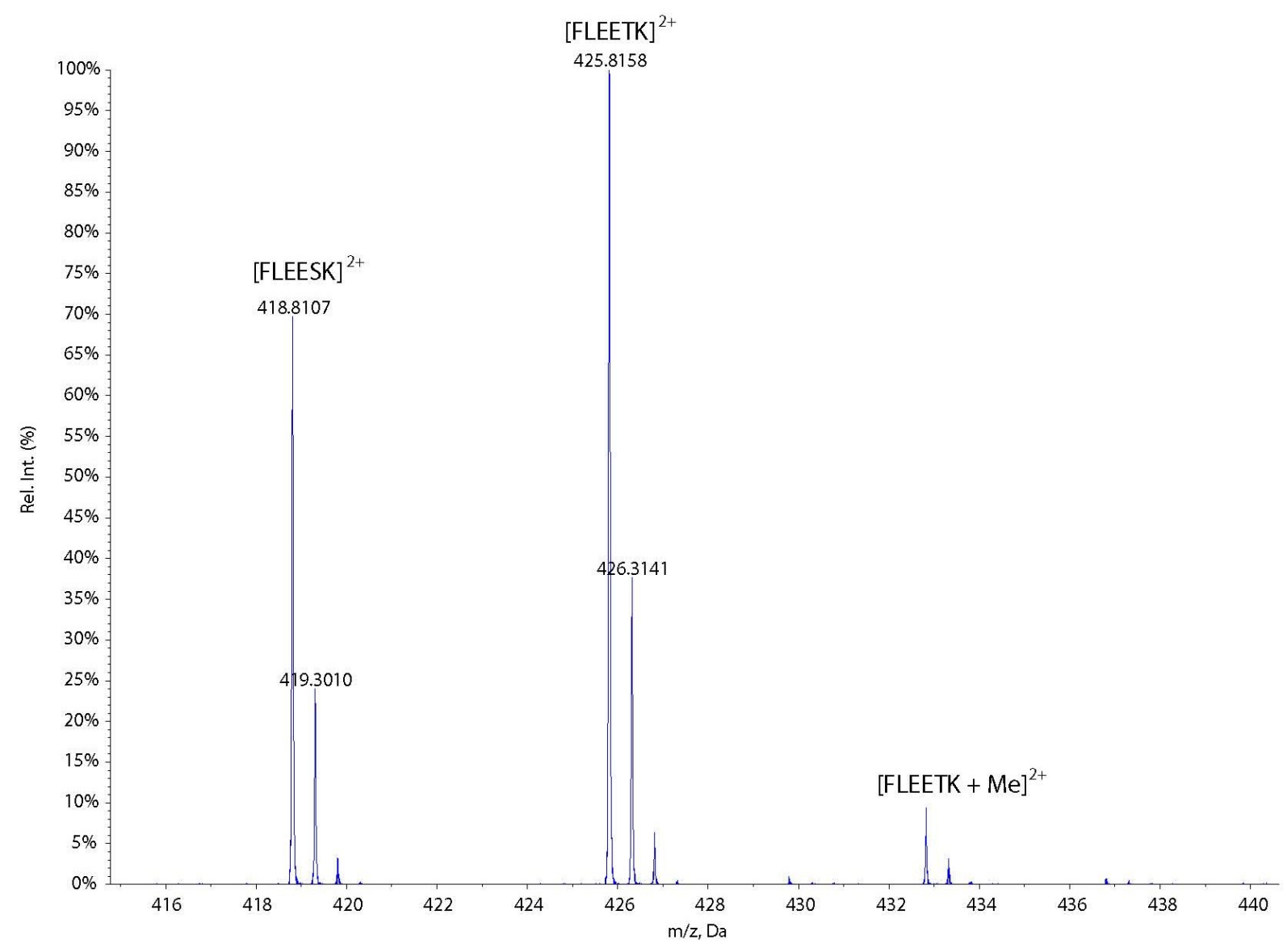

Figure 3.6: $M S$ analysis of FLEESK $(\mathrm{m} / \mathrm{z} 418.8)$ and FLEETK $(\mathrm{m} / \mathrm{z} 425.8)$ show that primary and secondary alcohol groups do not easily methylate (less than $10 \%$ ) within the time frame of our experiment.

Table 3.1: Summary of results for diazomethane-modified synthetic peptides

\begin{tabular}{|c|c|c|c|c|c|c|c|c|c|}
\hline \multirow{2}{*}{ Peptide } & \multicolumn{2}{|c|}{ Unmodified } & \multicolumn{2}{c|}{ Modified mass } & \multicolumn{2}{c|}{$\Delta \mathrm{m}$} & \multicolumn{3}{c|}{ Methylation } \\
\cline { 2 - 11 } & $\mathrm{m} / \mathrm{z}$ & $\begin{array}{c}\text { neutral } \\
\text { mass }\end{array}$ & $\mathrm{m} / \mathrm{z}$ & $\mathrm{Da}$ & $\mathrm{m} / \mathrm{z}$ & $\mathrm{Da}$ & $\begin{array}{c}\% \mathrm{~N}- \\
\text { terminus }\end{array}$ & $\begin{array}{c}\text { \# Me } \\
\text { groups }\end{array}$ & $\begin{array}{c}\# \text { fixed } \\
\text { charges }\end{array}$ \\
\hline [FLEEVK] $^{2+}$ & 382.7 & 763.4 & 424.7 & 849.4 & 42 & 86 & 100 & 6 & 2 \\
\hline$[\mathrm{FLEEVR}]^{2+}$ & 396.7 & 791.4 & 417.7 & 835.4 & 21 & 44 & 100 & 3 & 1 \\
\hline$[\mathrm{FLEENK}]^{2+}$ & 390.2 & 778.4 & 432.2 & 864.4 & 42 & 86 & 100 & 6 & 2 \\
\hline [FLEEYK] $^{2+}$ & 414.7 & 827.4 & 463.7 & 927.4 & 49 & 100 & 100 & 7 & 2 \\
\hline [FLEEHK] $^{3+}$ & 268.1 & 801.4 & 305.5 & 916.4 & 37.3 & 115 & 100 & 8 & 3 \\
\hline [FLEESK] $^{2+}$ & 376.7 & 751.4 & 418.8 & 837.6 & 42 & 86 & 100 & 6 & 2 \\
\hline [FLEETK] $^{2+}$ & 383.7 & 765.4 & 425.8 & 851.6 & 42 & 86 & 100 & 6 & 2 \\
\hline
\end{tabular}

* - percentage of $\mathrm{N}$-terminal amine quaternization 
Table 3.2: Summary of functional groups tested with TrEnDi and the resulting structure postTrEnDi modification

\begin{tabular}{|c|c|c|}
\hline Functional group & pKa & Observed affect post-TrEnDi \\
\hline & $\sim 10.6^{103}$ & primary amines and formation \\
\hline & of a fixed positive charge \\
\hline
\end{tabular}




\begin{tabular}{|c|c|c|}
\hline$Y^{\mathrm{OH}}$ & $\sim 12$ to & $\underbrace{\mathrm{OH}}$ \\
& & $13^{101,102}$ \\
of threonine side chain
\end{tabular}




\subsubsection{Tandem Mass Spectrometry of Methylated Peptides}

Fragmentation of the unmodified peptides yielded a variety of product ions resulting from peptide bond cleavage. Although $y$ - and $b$-ions provide sequence information, multiple fragmentation channels divide and therefore weaken the intensity of prospective MRM scans and make it more difficult to predict which parent to fragment ion transition would provide the most sensitivity. The fragmentation mechanisms of the diazomethane-methylated peptides were explored by $\mathrm{MS}^{2}$ analysis; peptides containing the trimethylated $\mathrm{N}$-terminus yielded reproducible and therefore predictable fragmentation patterns. Similar to previous studies on the fragmentation of peptides containing a permethylated $\mathrm{N}$-terminus, ${ }^{72} \mathrm{MS}^{2}$ confirmed that one of the most predominant fragmentation channels is the production of the $a_{2}$ ion; in this case $\mathrm{m} / \mathrm{z} 275.22$ (Figure 3.4b, Figure 3.7 and Figure $3.8 \mathrm{c}$ ). This $\mathrm{a}_{2}$ ion is preferentially formed due to the immobilization of charge on the $\mathrm{N}$-terminus. The significance of this consistent and dominant $a_{2}$ ion-forming fragmentation channel is that our chemistry enables a novel and powerful dimension to label free quantitation strategies: the ability to fully predict MRM transitions a priori, as discussed in greater detail below. The trimethylated lysine C-terminus also produced a fixed positive charge and a characteristic +42 Da shift on all lysine terminating $y$-ions. In general, this produced an enhanced intensity of lower mass $y$-ions in addition to the $\mathrm{a}_{2}$ ion, opening up additional potential MRM transitions per peptide that would lead to sensitive analyses in cases where isobaric interference hampers the use of a single MRM transition. 
As indicated in Table 3.1, two of the peptides were stably modified in locations other than primary amines. In the case of modified FLEEHK, a [FLEEHK] ${ }^{3+}$ ion appearing at $\mathrm{m} / \mathrm{z} 305.36$ was observed (Figure 3.8a). When unmodified, FLEEHK was observed to form both the $2^{+}$and $3^{+}$states due to the proton affinity of the histidine residue. This reveals another advantage of our derivatization strategy; peptides with a distribution of charge states are driven to a single state $\left(3^{+}\right.$in the case of FLEEHK) thereby enhancing the signal intensity. The fragmentation of $[\text { FLEEHK }]^{3+}$ yielded low mass $y$-ions that were different than those generated from typical protonated peptides in that their charge state was greater than one. Figure $3.8 \mathrm{c}$ reveals the $\mathrm{MS}^{2}$ spectrum of FLEEHK, where the $\mathrm{y}_{2}$ - and $\mathrm{y}_{3}$-ions were observed to be doubly charged. These ions also had a mass shift of $+70 \mathrm{Da}$, suggesting that the imidazole group on the histidine residue was dimethylated (presumably in a 1,3-fashion, thus preserving the aromaticity of the imidazolium ion) resulting in an additional fixed positive charge on the peptide. $\mathrm{MS}^{2}$ analysis of FLEEYK (Figure 3.8c) demonstrated the presence of a methylated hydroxyl group on the tyrosine side chain via the $y_{2^{-}}, y_{3^{-}}$and $y_{4}$-ions as the masses of all of these ions increased by +56 Da. 

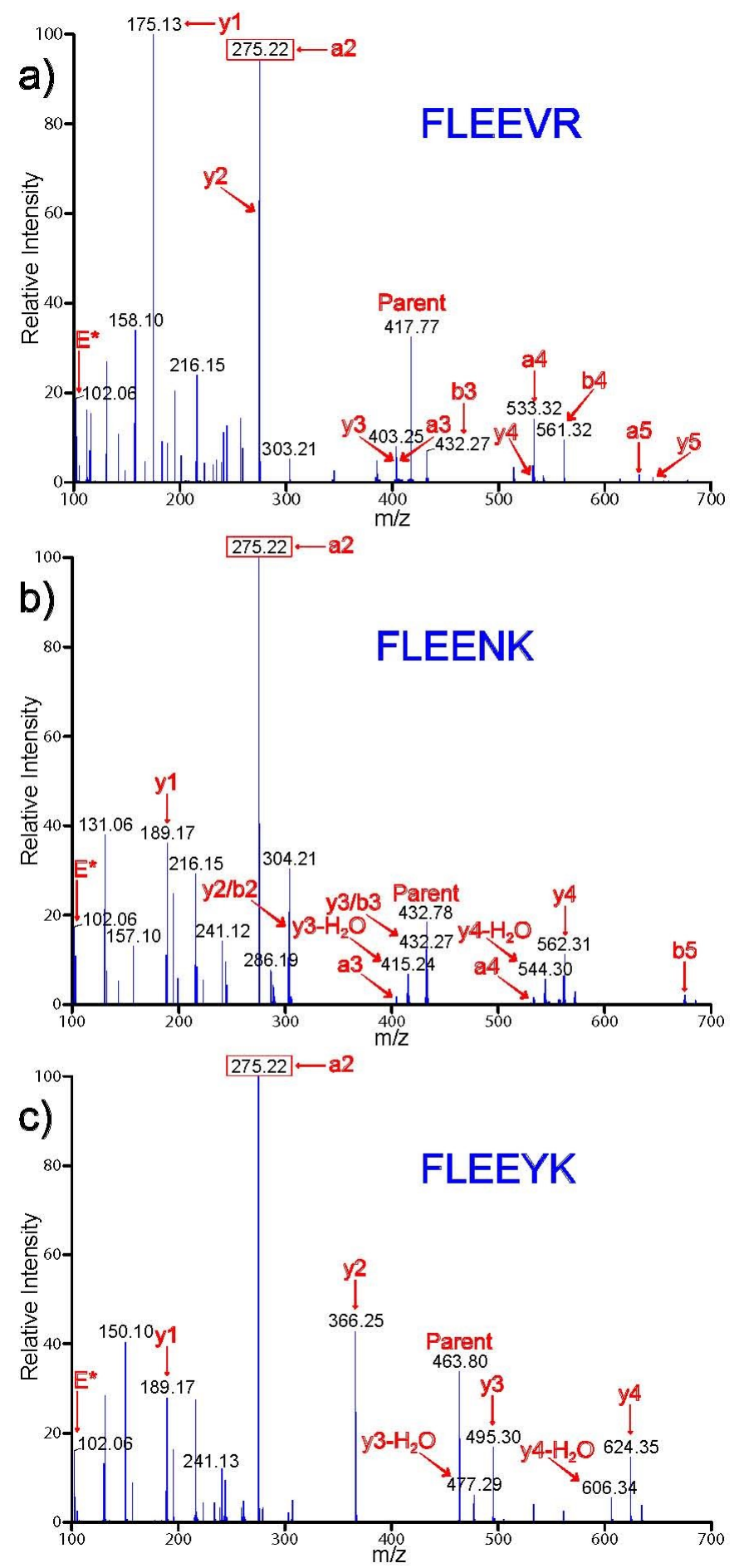

Figure 3.7: a) $\mathrm{MS}^{2}$ of TrEnDi-modified FLEEVR revealing that the guanidinium side chain of arginine does not get methylated. b) $\mathrm{MS}^{2}$ of TrEnDi-modified FLEENK revealing that the amide side chain of asparagine does not get methylated. c) $\mathrm{MS}^{2}$ of TrEnDi-modified FLEEYK revealing that the phenol side chain of tyrosine does get methylated. All ions fragment to form a y-ion series and a predominant $a_{2}$ fragment. 


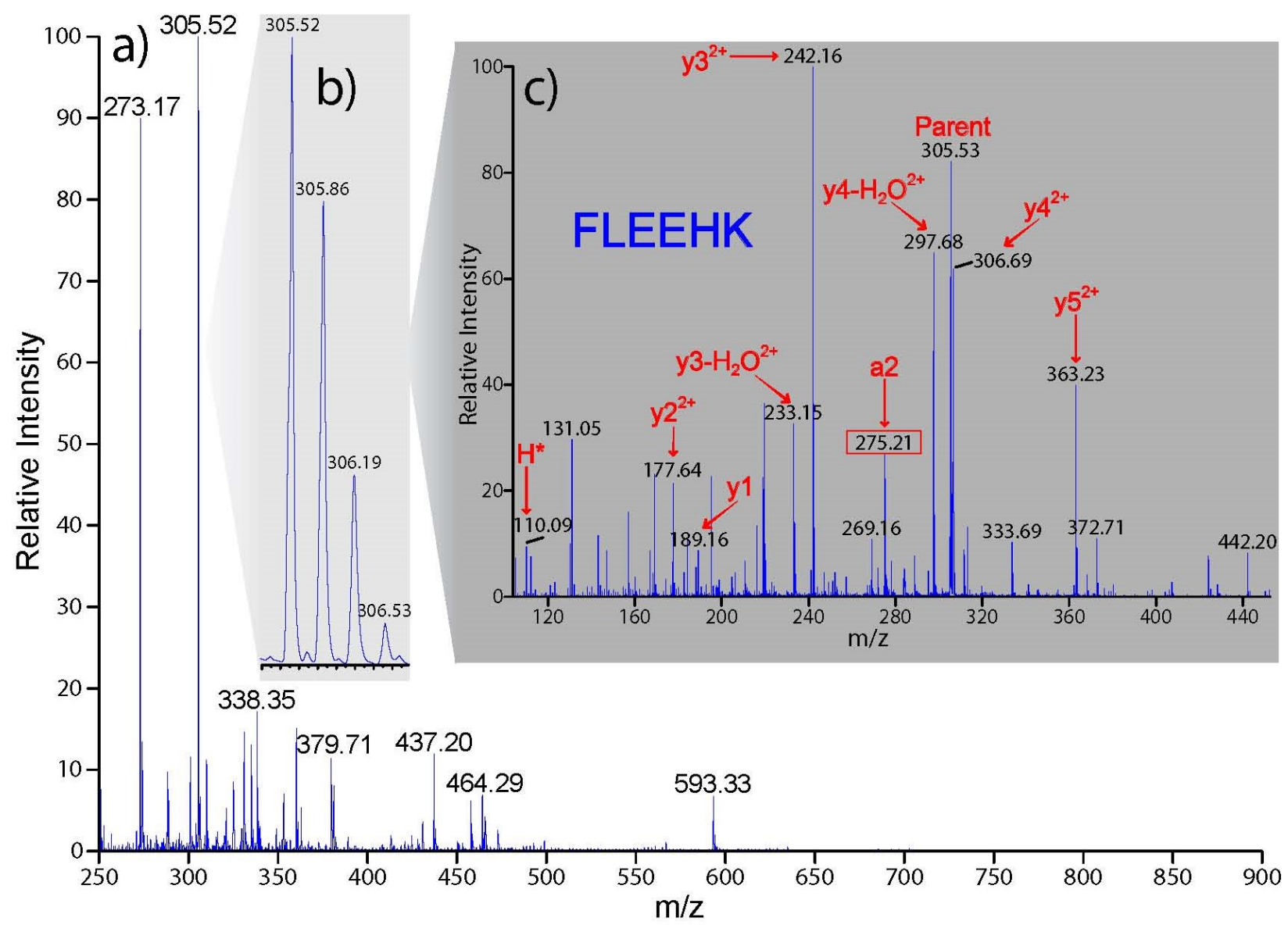

Figure 3.8: a) MS of TrEnDi-modified FLEEHK (m/z 305.52). b) This peptide is triply charged as revealed by the molecular ion isotopic spacing of $\Delta 0.33 \mathrm{Th}$. c) $\mathrm{MS}^{2}$ of TrEnDi-modified FLEEHK revealing $y$-ion series, a y-ion series with a water loss, the immonium ion of histidine and the characteristic $a_{2}$ fragment ion. 


\subsubsection{Multiple peptides tested simultaneously}

Our method demonstrated that peptides could be modified to a single stable product; however, it was unknown if there would be limitations to the methylation chemistry if a pool of several peptides were modified. To test this, a solution containing FLEEVK, FLEEVR, FLEENK and FLEEYK was acidified, bound to the SCX column and modified with the ethereal diazomethane solution to produce doubly charged species. FLEEHK was not selected for this experiment to avoid low $\mathrm{m} / \mathrm{z}$ matrix noise in the spectra (observed in Figure 3.8a). Our results demonstrated that the chemistry was equally effective on all peptides (Figure 3.9a); FLEEVK, FLEEVR, FLEENK and FLEEYK were all successfully methylated and the base hydrolysis of all the methyl esters was complete yielding MS signals of $\mathrm{m} / \mathrm{z} 424,417,432$ and 463 , respectively. $\mathrm{MS}^{2}$ on all 4 peptides confirmed the formation of the $\mathrm{m} / \mathrm{z} 275.22 \mathrm{a}_{2}$-ion and the characteristic +42 Da mass shift for all lysine-terminating $y_{1}$-ions. A +56 Da mass shift was visible for all $y_{2+n}$-ions in FLEEYK, due to methylation of the phenol group, while arginine and asparagine, as expected, were not modified. This experiment demonstrated that methylation of multiple different peptides was possible, opening the possibility of derivatizing more complex peptide mixtures. 


\subsubsection{IDA MS ${ }^{2}$ and MRM analyses}

Two MS methods were used to demonstrate the amenability of our methods to sensitive label free MS analysis. The first method employed a typical abundance-based IDA MS ${ }^{2}$ experiment to analyze TrEnDi-modified peptides followed by an MRM experiment on the same sample. TrEnDi-modified FLEEVK, FLEEVR, FLEEYK and FLEENK were spiked into a peptide mixture ("protmix") that was three orders of magnitude greater in concentration and the ability of an MRM method to obtain quantitative data was assessed. To ensure accurate data, a second set of parent to abundant fragment ion transitions were performed for each peptide (Table 3.3).

Table 3.3: Summary of MRM transitions for TrEnDi modified peptides

\begin{tabular}{|c|c|c|}
\hline Modified peptide & $\mathrm{m} / \mathrm{z}$ parent & $\mathrm{m} / \mathrm{z}$ fragment (identity) \\
\hline \multirow{2}{*}[\text{FLEEVR]}]{$^{2+}$} & 417.7 & $275.2\left(a_{2}\right)$ \\
\hline & 417.7 & $533.3\left(a_{4}\right)$ \\
\hline \multirow{2}{*}[\text{FLEEVK]}]{$^{2+}$} & 424.7 & $275.2\left(\mathrm{a}_{2}\right)$ \\
\hline & 424.7 & $288.2\left(y_{2}\right)$ \\
\hline \multirow{2}{*}[\text{FLEENK}]{$^{2+}$} & 432.7 & $275.2\left(\mathrm{a}_{2}\right)$ \\
\hline & 432.7 & $189.1\left(\mathrm{y}_{1}\right)$ \\
\hline \multirow{2}{*}[\text{FLEEYK]}]{$^{2+}$} & 463.7 & $275.2\left(a_{2}\right)$ \\
\hline & 463.7 & $366.2\left(y_{2}\right)$ \\
\hline
\end{tabular}

The IDA MS ${ }^{2}$ experiment yielded many protmix protein identifications yet none of the FLEEXX peptides were selected for $\mathrm{MS}^{2}$ due to their low abundance; visual inspection of the expected masses via extracted ion chromatograms yielded no observable signal (data not shown). However, the MRM method was able to detect all four of the modified peptides from within the complex sample (Figure 3.9b). FLEEVK and FLEEVR were not chromatographically 
distinguishable, but were easily identifiable from the MRM traces. The lower intensity of the FLEENK trace is attributable to losses of this peptide in the C18 Zip-tip cleanup step; subsequent analyses of the salty solution post-cleanup revealed remnants of FLEENK presumably due to the relative polarity of this peptide. Our results demonstrate that TrEnDi-modified peptides produce strong MRM transitions and remain identifiable at low concentrations in the context of more complex peptide samples.
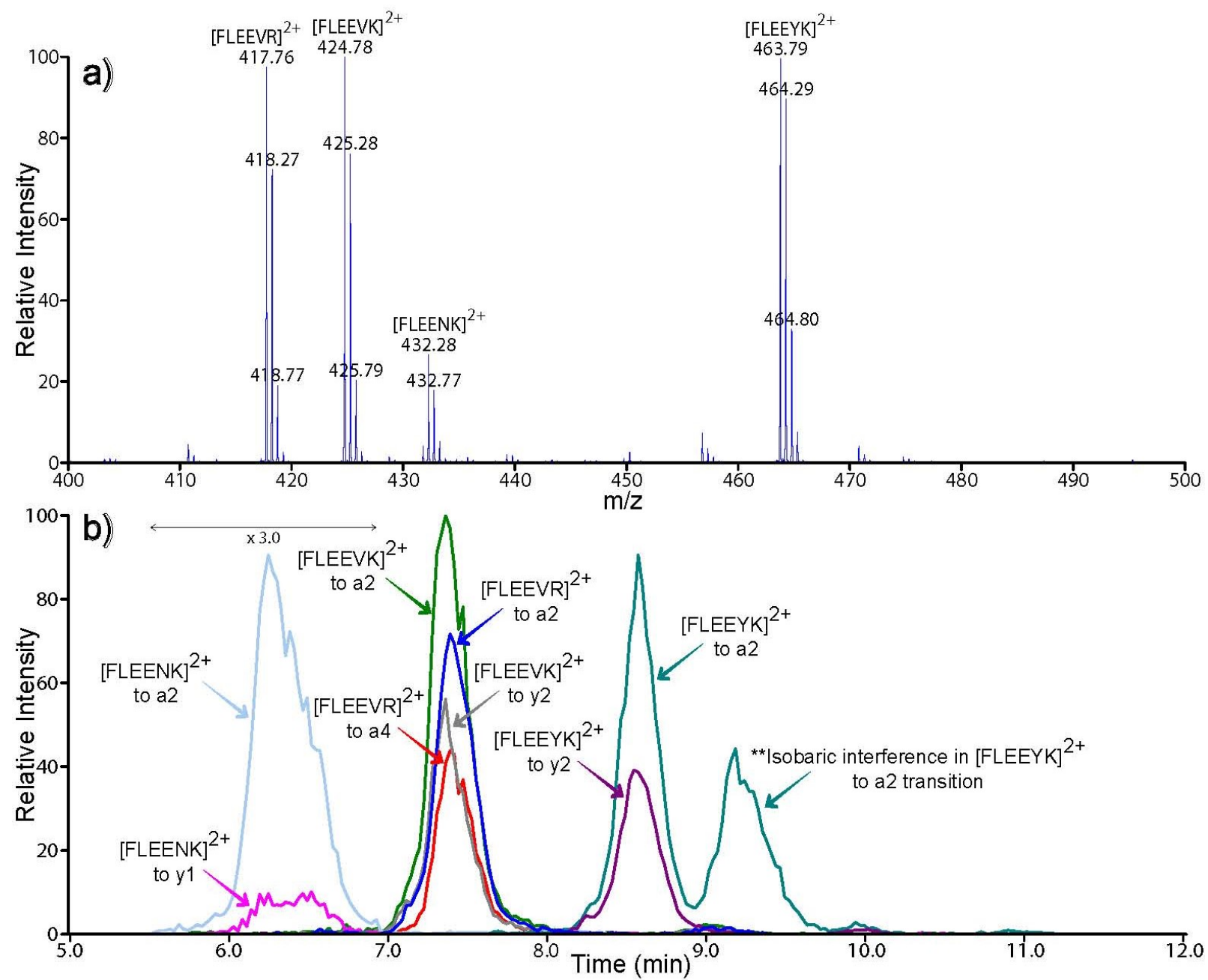

Figure 3.9: a) MS spectrum of a mixture of TrEnDi-modified FLEEVR, FLEEVK, FLEENK and FLEEYK. b) MRM chromatogram of these same four peptides spiked into a concentrated complex mixture of digested protmix (see Section 3.6) and successfully identified by two distinct parent ion to fragment ion transitions including the $a_{2}$ fragment ion in every case. 


\subsubsection{Diazomethane efficiency on a protein digest}

In order to further test the modification efficiency and the applicability of TrEnDi to quantitative proteomic analyses, the chemistry was tested on a solution of BSA. The protein was tryptically digested and analyzed via the AB Sciex 4000 Qtrap in IDA $\mathrm{MS}^{2}$ mode. Peptide identification was achieved using a Mascot search against the NCBInr database, as described in the methods. The Mascot results demonstrated that our experiment identified $52 \%$ of the total sequence of BSA. However, even when considering the low scoring peptide hits, $48 \%$ of the protein remained unidentifiable via IDA MS². This may have been due to low ionization efficiency or low proton affinity of those peptides. One randomly selected peptide, AEFVEVTK (highlighted in Figure 3.10), that was not identified by Mascot but had a favourable length with a characteristic lysine C-terminus, was targeted for further analysis. $5 \mu \mathrm{g}$ of digested BSA was bound to the SCX column and $450 \mu \mathrm{L}$ of ethereal solution containing $0.23 \mathrm{M}$ diazomethane was passed over the peptides to ensure complete methylation chemistry. Based on the pKa of the amino acid residues, the total mass shift due to methylation of our selected peptide was expected to be $+84 \mathrm{Da}$, while the $\mathrm{a}_{2}$ fragment was calculated to be $215.1266 \mathrm{Da}$. The MRM transition pair of $\mathrm{m} / \mathrm{z} 503.8$ to 215.1 at unit resolution was chosen for the detection of AEFVEVTK. As shown in Figure 3.10, the peptide was detected with considerable signal approximately 9 minutes into the analysis. The formation of the trimethylated $\mathrm{N}$-terminus via diazomethane poses a significant advantage for MRM analysis because it provides predictable derivatization and fragmentation behaviour, which allows for MRM analyses of previously unobserved peptides from a protein digest. 
Using a microfluidic device, the derivatization of tryptic peptides to form fixed, permanent positive charges using diazomethane has been demonstrated. Modified peptides show predictable fragmentation behaviour that is well suited for MRM quantitation strategies with enhanced sensitivity due to both the intensity of the $a_{2}$ fragment ion as well as the fact that derivatization drives each peptide to a uniform charge state, as observed in the case of FLEEHK. The modified peptides also display improved ionization characteristics including the ability to be observed when electrosprayed in aprotic solvents. TrEnDi-modified FLEEVK was dissolved in pure acetonitrile and showed a strong ESI-MS signal when analyzed (data not shown). Eliminating the need for a proton source in ESI obviates concerns regarding ion suppression due to differences in peptide proton affinities and opens up the possibility of employing novel MS analyses such as in-source ion mobility using molecules with high proton affinities (i.e. "proton scrubbers") or the use of chromatographic schemes with nonconventional, aprotic mobile phases. 


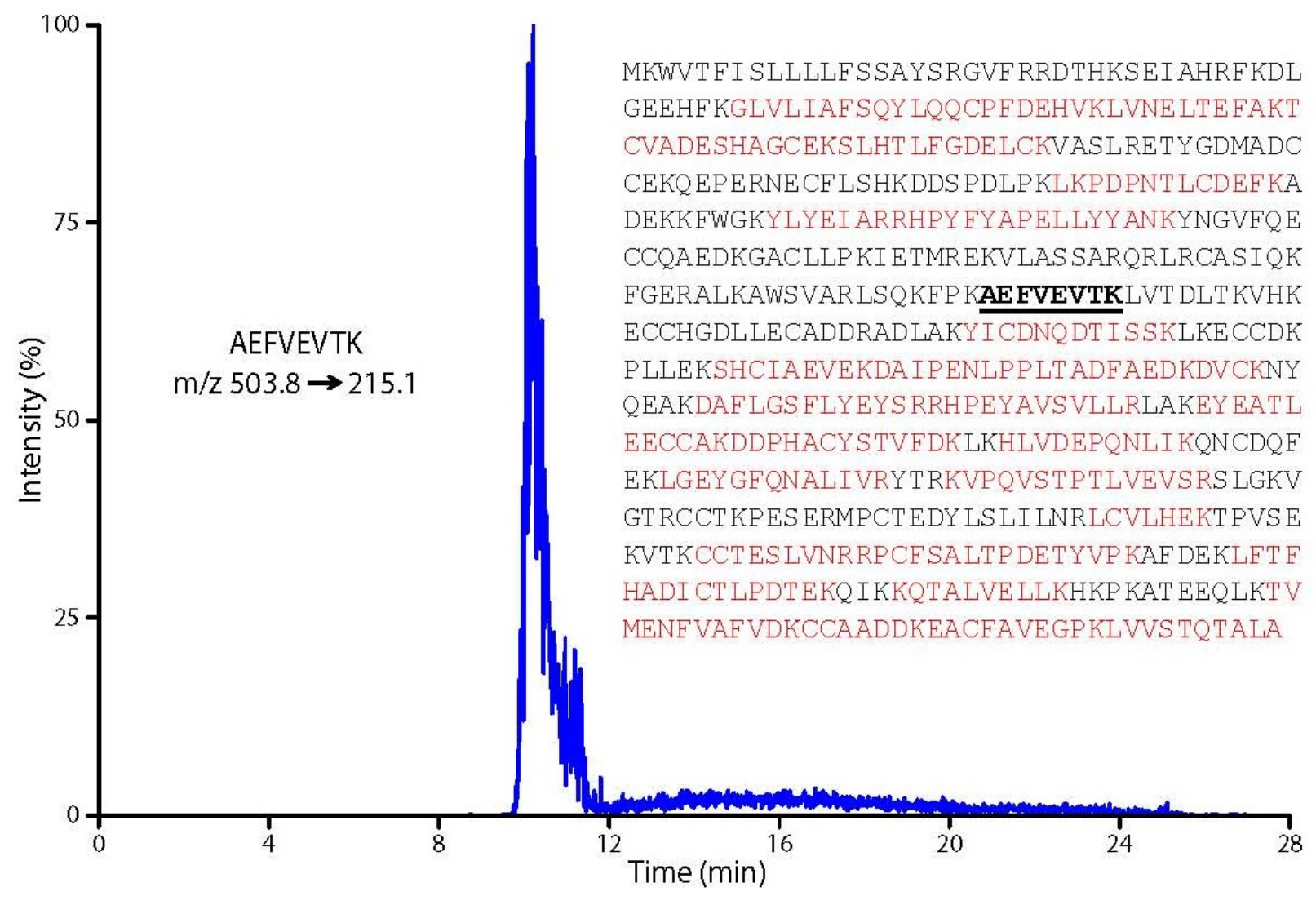

Figure 3.10: MRM chromatogram of BSA peptide AEFVEVTK with an inset of the BSA amino acid sequence. The regions identified by Mascot on data acquired using an IDA experiment are shown in red and the peptide AEFVEVTK that was successfully identified using TrEnDi is underlined in bold font. 


\subsection{Conclusion}

The trimethylation enhancement using diazomethane (TrEnDi) technique has been successfully developed on small peptides. Methylation has been shown to occur on functional groups with pKa values lower than 11 and produce quaternary ammonium groups on lysine side chains, peptide $\mathrm{N}$-termini and imidazole groups. Phenol groups were also shown to be methylated (without adding a fixed positive charge); however, amide and guanidinium groups were not methylated by our strategy. All carboxylic acids were methylated using this chemistry and the methyl esters were easily revered back to acids with the use of a base.

TrEnDi modifies peptides such that they contain fixed, permanent positive charges, increasing the sensitivity of ESI MS analysis by driving the charge state of each peptide to a single value and opening the possibility of using aprotic solvents. The derivatization chemistry is predictable but equally important is that the fragmentation behaviour of TrEnDi-modified peptides is also predictable. TrEnDi-modified peptides demonstrate a propensity to fragment and form the $a_{2}$ fragment ion. In doubly charged ions, the $a_{2}$ fragment was the most intense fragment revealing how this chemistry is able to enhance the sensitivity of MRM analyses. The proof of this sensitivity gain was observed in the analysis of an unobserved peptide from BSA using conventional proteomics methods. TrEnDi places a fixed positive charge on peptides and induces predictable fragmentation behaviour, permitting "blind" MRM experiments and providing a mechanism to observe and quantify elusive peptides. 


\subsection{TrEnDi on lipids}

Lipids play an important role in a number of physiological functions. Their participation ranges from various signal transduction pathways, ${ }^{106,107}$ proliferation, ${ }^{108}$ and apoptosis, ${ }^{109,110}$ to membrane trafficking in the cell. ${ }^{111,112}$ Lipids have also been associated with a number of neurodegenerative diseases, ${ }^{113,114}$ diabetes ${ }^{115}$ and even cancer. ${ }^{116}$ Therefore, a lot of separation and analysis techniques have been implemented over the years to study lipids, including mass spectrometry. Advances in ionization techniques ${ }^{5,6,40,41}$ has allowed researchers to use mass spectrometry to probe deeper into the realm of lipidomics and study the various combinations in their polar head groups, their fatty acyl chains and their general backbone stuctures. ${ }^{8}$

Lipids have unique head groups that vary in their proton affinities. This means that upon analysis using ESI-MS, the relative intensities of different lipids in a single mass spectrum would not necessarily correlate to their concentrations; as a result, different quantitation strategies have been developed. Many quantitation methods involve the incorporation of internal standards with stable isotopes ${ }^{117}$ such as ${ }^{2} \mathrm{H}$ and ${ }^{13} \mathrm{C}$. Stable isotopes may be introduced chemically ${ }^{118}$ or metabolically, ${ }^{119}$ permitting quantitation via comparing the relative intensities of each isotopically labeled lipid in a mass spectrum. These methods use the isotopically labeled internal standard, identical to the lipid of interest, so that different samples may be simultaneously analyzed but have distinctive different masses as measured by mass spectrometry. Since both lipids will have the same proton affinities, they will ionize equally, the difference in their relative intensities can be measured and the concentration of the target lipid 
can be determined. Methods that use ${ }^{2} \mathrm{H}$ labeled lipid standards for MS/MS analysis have been known to have differences in the response compared to the native compounds due to kinetic isotope effects in the fragmentation reactions, ${ }^{8}$ therefore a complete calibration curve is often required when performing deuterium labeled lipid MS/MS analysis. Isotopic labeling of standards is a very useful technique; however, it can be quite expensive.

As an alternative to isotopic labeling, internal standard strategies using compounds that are closely related in structure and physiochemical properties to the target lipids (such as nonnaturally occurring lipid standards) have also been developed for lipid quantitation. ${ }^{8}$ A known amount of non-naturally occurring lipids, which have similar physiochemical properties as the target lipids, can be spiked into the sample of target lipids as a means of normalization. Although this particular strategy offers a cheaper alternative to isotopic labeling, one of the disadvantages is that this method is only applicable to a limited number of lipids in complex mixtures. ${ }^{8,120,121}$ Furthermore, there are some lipid classes that are detected more sensitively than others, potentially leading to suppression effects when complex mixtures are analyzed; ${ }^{122}$ highlighting a need for new analytical tools in this area.

This thesis work has developed a novel strategy that harnesses the benefits of diazomethane to enhance lipid sensitivity. In this chapter, we report a fast and cost-effective in solution strategy that results in the complete methylation of phosphate moieties, carboxylic acids and primary amines, ultimately rendering target lipids such as PE and PS to be positively charged. Moreover, previous research on lipids bearing fixed positive charges has demonstrated the preferential formation of a charged lipid head group ion upon collisional 
activation. $^{8,58}$ This translates into the ability to conduct predictable and highly sensitive precursor ion analyses and therefore more robust, sensitive, precise and accurate label-free quantitative lipidomic analyses.

\subsection{Sample preparation}

Each of the PE (16:0, 18:1), PC (18:1, 14:0) and SM (18:1/16:0) lipids were dried using nitrogen gas and resuspended in ethanol. PS (18:0, 18:2) was purchased as a powder and was immediately dissolved in ethanol. All lipid standard concentrations ranged from $3.4 \mathrm{mM}$ to 10 $\mathrm{mM}$.

\section{2 $N$-methyl- $N$-nitroso- $p$-toluenesulfonamide (Diazald) production}

See section 2.1

\subsection{Diazomethane production}

See section 2.2 


\subsection{In solution chemical derivatization}

$2 \mathrm{~mL}$ clear screw vials were used in all experiments. Before each experiment, the vials were flushed with nitrogen gas for approximately 2 minutes. $10 \mu \mathrm{L}$ of $\mathrm{EtOH}$ was added into each vial followed by $15 \mathrm{nmol}$ of lipid. Before each experiment, a 14:1 solution of ether and $\mathrm{HBF}_{4}$ was freshly prepared and mixed vigorously. $0.5 \mu \mathrm{L}$ of the $14: 1$ solution was added to the vial containing the lipid standard. Lipids were derivatized by adding enough of the ethereal solution containing diazomethane to the vials such that the colour remained yellow (approximately 250$300 \mu \mathrm{L})$. The solution was carefully swirled followed by immediate drying under nitrogen gas. Once completely dried, the modified lipids were resuspended in $100 \mu \mathrm{L} \mathrm{EtOH}$.

\subsection{ESI-MS and MS² for individual lipids}

$3 \mu \mathrm{L}$ of the resuspended solution was inserted into a Proxeon nanoelectrospray emitter followed by direct analysis of the methylated lipids using an AB Sciex QStar XL mass spectrometer equipped with a nanoESI source. Spectra were obtained using a nanoESI voltage

of $1000 \mathrm{~V}$, declustering and focusing potentials at $30 \mathrm{~V}$ and $120 \mathrm{~V}$, respectively and an $\mathrm{MS}^{2}$ collision energy for $\mathrm{MS}^{2}$ experiments at $40 \mathrm{eV}$ and $60 \mathrm{eV}$ for PS studies. 


\subsection{Sensitivity test for individual lipids}

After verifying that the sample was completely methylated, an equimolar amount of unmodified lipid was added to the resuspended solution containing modified lipid. $3 \mu \mathrm{L}$ of the resuspended equimolar solution of modified and unmodified lipid was inserted into a Proxeon nanoelectrospray emitter followed by direct analysis of the methylated lipids using the QqTOF MS equipped with nanoESI source. Spectra were obtained using a nanoESI voltage of $1000 \mathrm{~V}$, declustering and focusing potentials at $30 \mathrm{~V}$ and $120 \mathrm{~V}$, respectively and an $\mathrm{MS}^{2}$ collision energy for $\mathrm{MS}^{2}$ experiments that ranged between 40 and $60 \mathrm{eV}$.

\subsection{Shotgun lipidomics Precursor ion scanning for multiple lipids}

A $12 \mu \mathrm{L}$ solution containing equimolar amounts of unmodified and modified PC, PS, PE and SM lipids were directly injected via electrospray emitter. Lipids were analyzed via EMS and precursor ion scanning modes on the AB Sciex 4000 QTRAP. In the precursor ion scanning mode, lipid identification was accomplished by monitoring for the $\mathrm{m} / \mathrm{z} 184$ fragment of the unmodified PC and SM species as well as the TrEnDi modified fragment of $\mathrm{m} / \mathrm{z} 198$. The sensitivity increase gained from TrEnDi modified PE was explored by comparing the neutral loss scanning of 141 for the unmodified PE versus the 198 fragment post TrEnDi. The sensitivity gained from TrEnDi modified PS was also explored by comparing the neutral loss scanning for $\mathrm{m} / \mathrm{z} 185$ for the unmodified PS with the precursor ion scanning of the dominant 144 and 256 
fragments for the TrEnDi modified PS (listed in Table 4.2 below). Spectra were obtained using an ESI voltage of $5000 \mathrm{~V}$, a declustering potential of $40 \mathrm{~V}$ and a collision energy of $40 \mathrm{eV}$ for all unmodified and TrEnDi-modified lipids except modified PS; to maximize signal, TrEnDi-modified PS required a collision energy of $60 \mathrm{eV}$.

\subsection{Results and discussion for TrEnDi lipidomics}

Diazomethane has been previously used to methyl esterify fatty acids for GC analysis. $^{63,64,65}$ It has also been used to convert dipalmitoyl glycerophosphoethanolamine to pure dipalmitoyl glycerophospho $\left[\mathrm{N}-\right.$ methyl- $\left.{ }^{3} \mathrm{H}\right]$ choline in solution, ${ }^{67}$ however it has not been shown to successfully methylate both the primary amines and phosphate moieties found in certain lipids. Trimethylsilyl diazomethane has also been used to methylate lipids, ${ }^{74}$ though in this case only the phosphate moieties and carboxylic acids were modified, leaving other functional groups such as primary amines unmodified. Recent Smith lab studies about the reactivity of diazomethane have shown that a variety of functional groups can be simultaneously methylated when placed in close proximity to diazomethane, including primary amines to produce an ammonium ion (Chapter 3 ). An attractive feature of lipid primary amine methylation is that a permethylated lipid would contain a fixed, permanent positive charge via a quaternary ammonium ion, enhancing ionization efficiency and sensitivity when analyzed by MS, especially when one can neutralize all negative charges via methylation of the negatively charged oxygen on the phosphate group. The method we have developed is successful at fully 
methylating lipids (such as PE, PC, SM and PS) when they are subjected to very small amounts of diazomethane in the presence of tetrafluoroboric acid $\left(\mathrm{HBF}_{4}\right) . \quad \mathrm{HBF}_{4}$ is the key to the success of in-solution lipid-based TrEnDi because it is extremely acidic, enabling the protonation of the lipids of interest, but also produces a non-coordinating counterion. This ultimately prevents diazomethane from methylating the conjugate base of the acid, thereby directing all methylation towards the lipids (See Figure 4.1). Figure 4.1 illustrates the reaction of diazomethane with a primary amine; acidic ammonium ion protons protonate diazomethane, creating an unstable diazonium cation, which carries out an $\mathrm{S}_{\mathrm{N}} 2$ reaction methylating the amine while releasing $\mathrm{N}_{2}$ gas. Critically, this reaction produces a fixed positive charge on the lipid via the formation of a quaternary ammonium ion while neutralizing the negative charge via the formation of the phosphate methyl ester. 


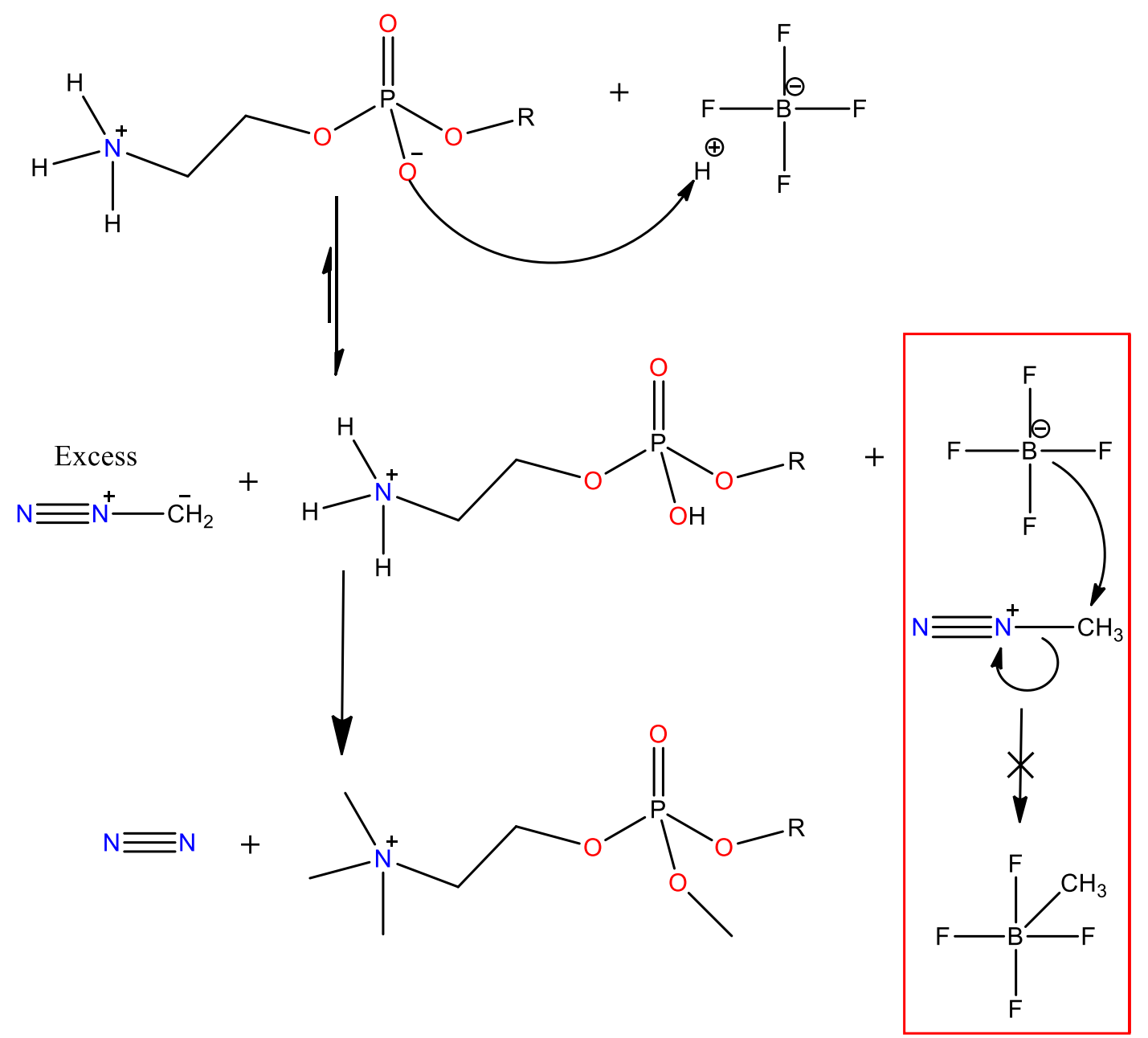

Figure 4.1: Methylation of a phosphoethanolamine lipid head group with diazomethane

Initially, a lipid standard 1-palmitoyl-2-oleoyl-sn-glycero-3-phosphoethanolamine [PE(16:0-18:1)] was chosen to study the chemistry between diazomethane and the phosphoethanolamine (PE) head group in solution. The mass spectra of the unmodified lipid and the lipid following treatment with diazomethane are shown in Figure 4.2 and Figure 4.3, respectively. Figure 4.2 a shows that the unmodified PE signal is divided into two parent ions: the protonated ion $[\mathrm{PE}+\mathrm{H}]^{+}$at $\mathrm{m} / \mathrm{z} 718.52$ and $[\mathrm{PE}+\mathrm{Na}]^{+}$at $\mathrm{m} / \mathrm{z}$ 740.50. Tandem mass spectrometry $\left(\mathrm{MS}^{2}\right.$ ) analysis of $[\mathrm{PE}+\mathrm{H}]^{+}$at $\mathrm{m} / \mathrm{z} 718.52$ (Figure $4.2 \mathrm{~b}$ ) shows several fragmentation 
channels yielding a variety of product ions resulting from various bond cleavages. Figure 4.3a reveals the fully modified $[\mathrm{PE}]^{1+}$ ion at $\mathrm{m} / \mathrm{z}$ 774.58; a total mass shift of $+56 \mathrm{Da}$ from the unmodified $[\mathrm{PE}+\mathrm{H}]^{+}$, indicating that the lipid had been methylated four times. The fragmentation mechanism of the diazomethane-methylated PE was explored by $\mathrm{MS}^{2}$ analysis (Figure 4.3b); PE subjected to TrEnDi yielded vastly simpler, reproducible and therefore predictable fragmentation patterns.

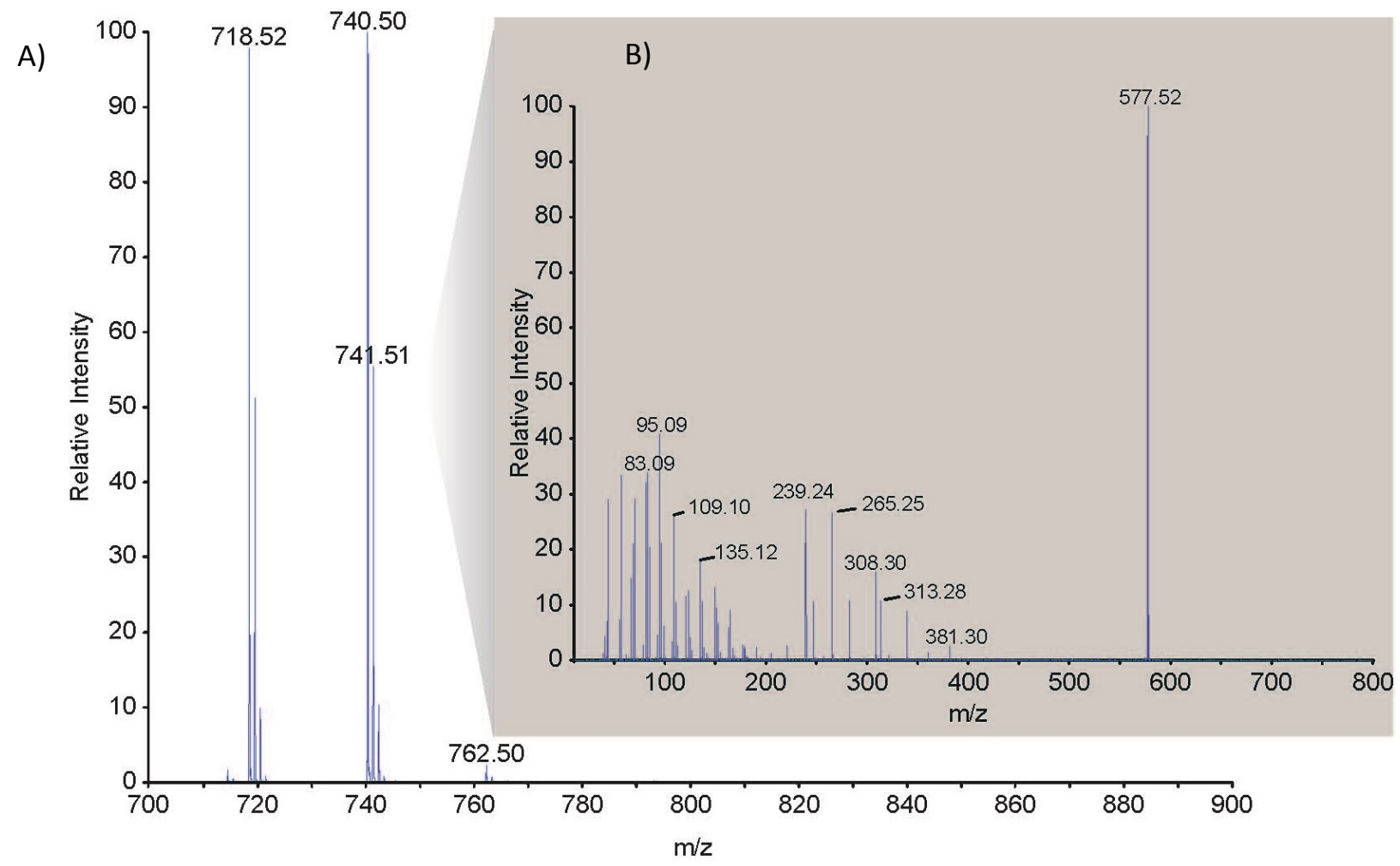

Figure 4.2: a) PE unmodified revealing protonated and sodiated parent ions. b) Fragmentation of the unmodified PE indicates divided signal along the various fragmentation channels. 


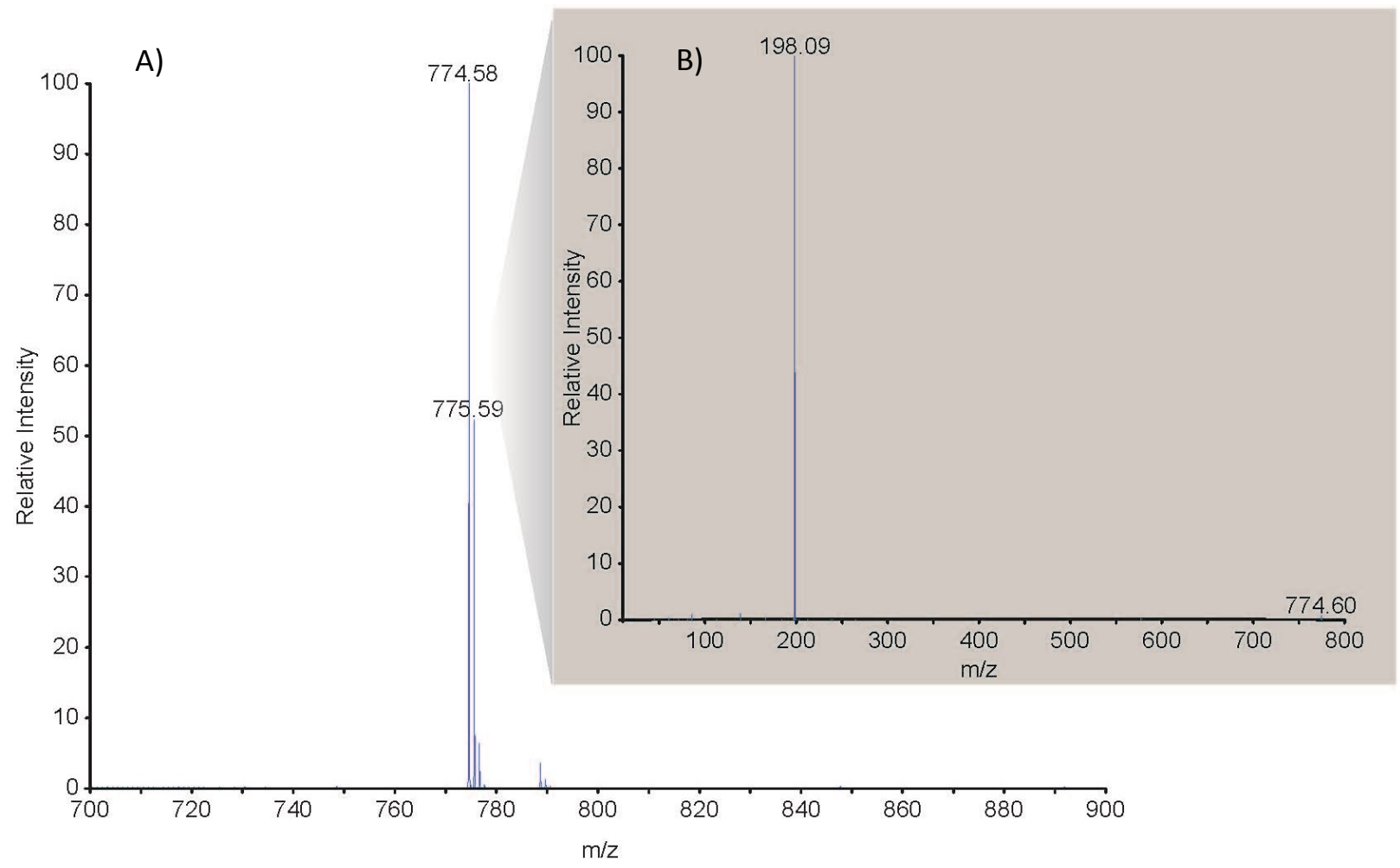

Figure 4.3: a) PE treated with diazomethane reveals four methyl groups ( $\mathrm{m} / \mathrm{z} 774.6)$. b) Fragmentation of this lipid revealed a single fragmentation channel $(\mathrm{m} / \mathrm{z} 198.1)$

$\mathrm{MS}^{2}$ confirmed that the location of these four newly inserted methyl groups was inserted on the head group, driving the fragmentation channels to one product at $\mathrm{m} / \mathrm{z} 198.09$; likely due to the immobilization of charge on the newly formed quaternary ammonium ion. While it was predicted that the primary amine will trimethylate with diazomethane, our studies indicated that a fourth methylation reaction was also occurring at the phosphate oxygen, which is why a mass shift of 14 Da was observed over the predicted $\mathrm{m} / \mathrm{z} 184$ phosphocholine head group. The significance of this consistent and dominant fragmentation channel is that our chemistry enables a novel and powerful dimension to label free quantitation strategies for PE lipids: the ability to fully acquire the precursor ion transition of PE lipids. The derivatization chemistry and structure of TrEnDi modified PE is illustrated in Figure 4.4. 


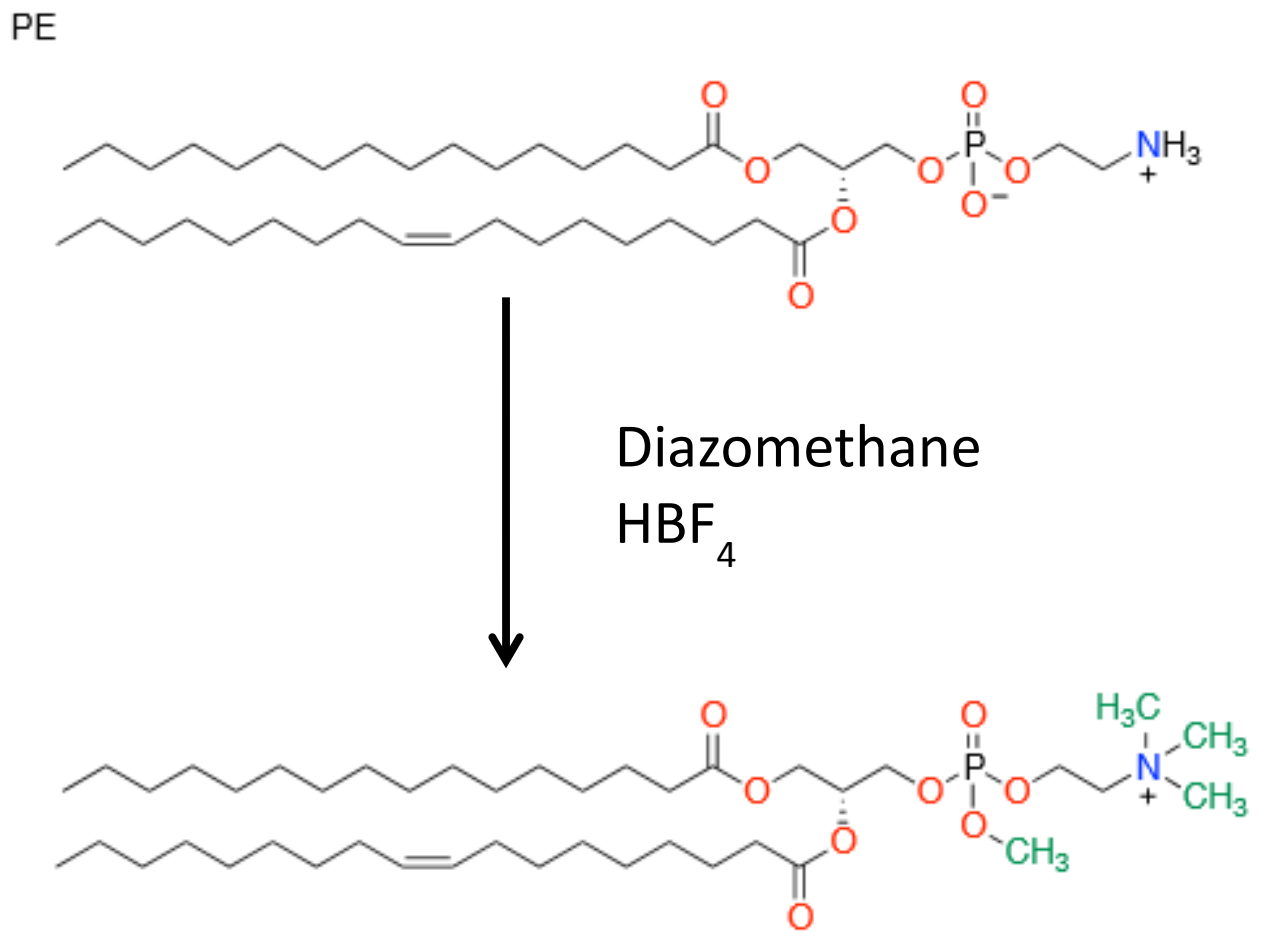

Figure 4.4: Fully modified PE (16:0-18:1) via treatment with diazomethane and $\mathrm{HBF}_{4}$

In further studies to determine the efficiency of TrEnDi, 1-octadecanoyl-2-(9Z,12Zoctadecadienoyl)-sn-glycero-3-phospho-L-serine(sodium salt) [PS(18:0/18:2(9Z,12Z))] was also evaluated. Figure 4.5 and Figure 4.6 reveal the mass spectrum of the unmodified PS lipid and PS following treatment with diazomethane. When unmodified, PS was observed to form multiple parent ions due to cationization with $\mathrm{Na}^{+}$(Figure 4.5a) while the $\mathrm{MS}^{2}$ analysis indicated a much divided signal amongst all the fragmentation channels, therefore weakening the intensity of prospective scanning modes (Figure $4.5 \mathrm{~b}$ ). 


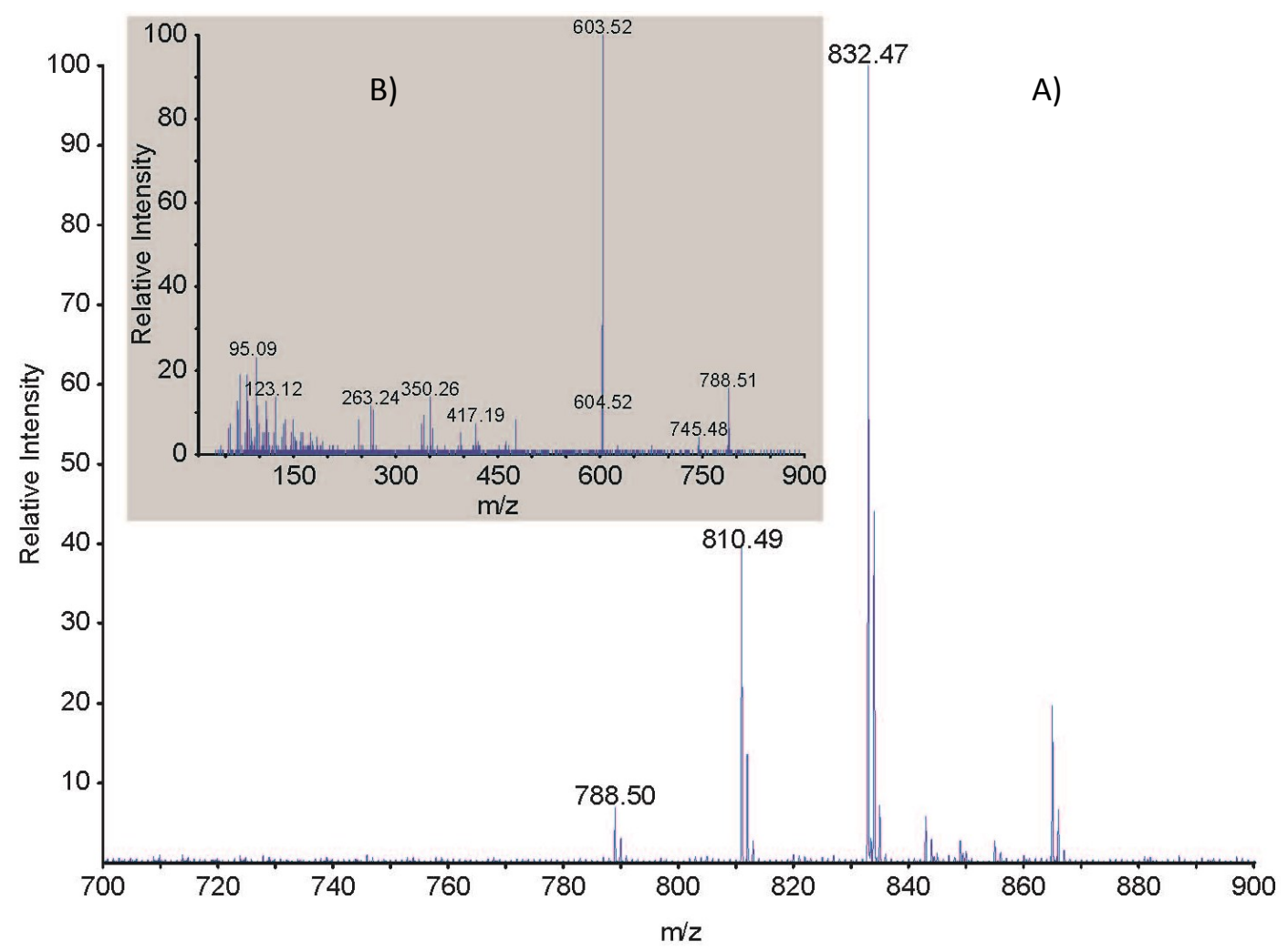

Figure 4.5: a) PS unmodified revealing three parent ions (protonated, sodiated and doubly sodiated). b) Fragmentation of the unmodified PS at $\mathrm{m} / \mathrm{z} 788.5$ indicates divided signal along the various fragmentation channels.

In the case of diazomethane-modified PS, the mass spectrum reveals that five methylations are observed, confirmed by the dominant molecular ion $[P S]^{1+}$ at $\mathrm{m} / \mathrm{z} 858.59$ (Figure 4.6a). This reveals another advantage of our derivatization strategy, a lipid with a distribution of parent ions is driven to a single parent ion thereby enhancing the signal intensity. Trimethylation of the primary amine, methylation of the negatively charged oxygen in the phosphate head group as well as the carboxylic acid was confirmed by $\mathrm{MS}^{2}$ analysis (Figure 4.6b); with two dominant fragmentation channels shown at $\mathrm{m} / \mathrm{z} 256.09$ and 144.10 respectively. The overall reaction of PS with diazomethane and $\mathrm{HBF}_{4}$ is illustrated in Figure 4.7 and proved to drive the chemistry to a single, stable product. 


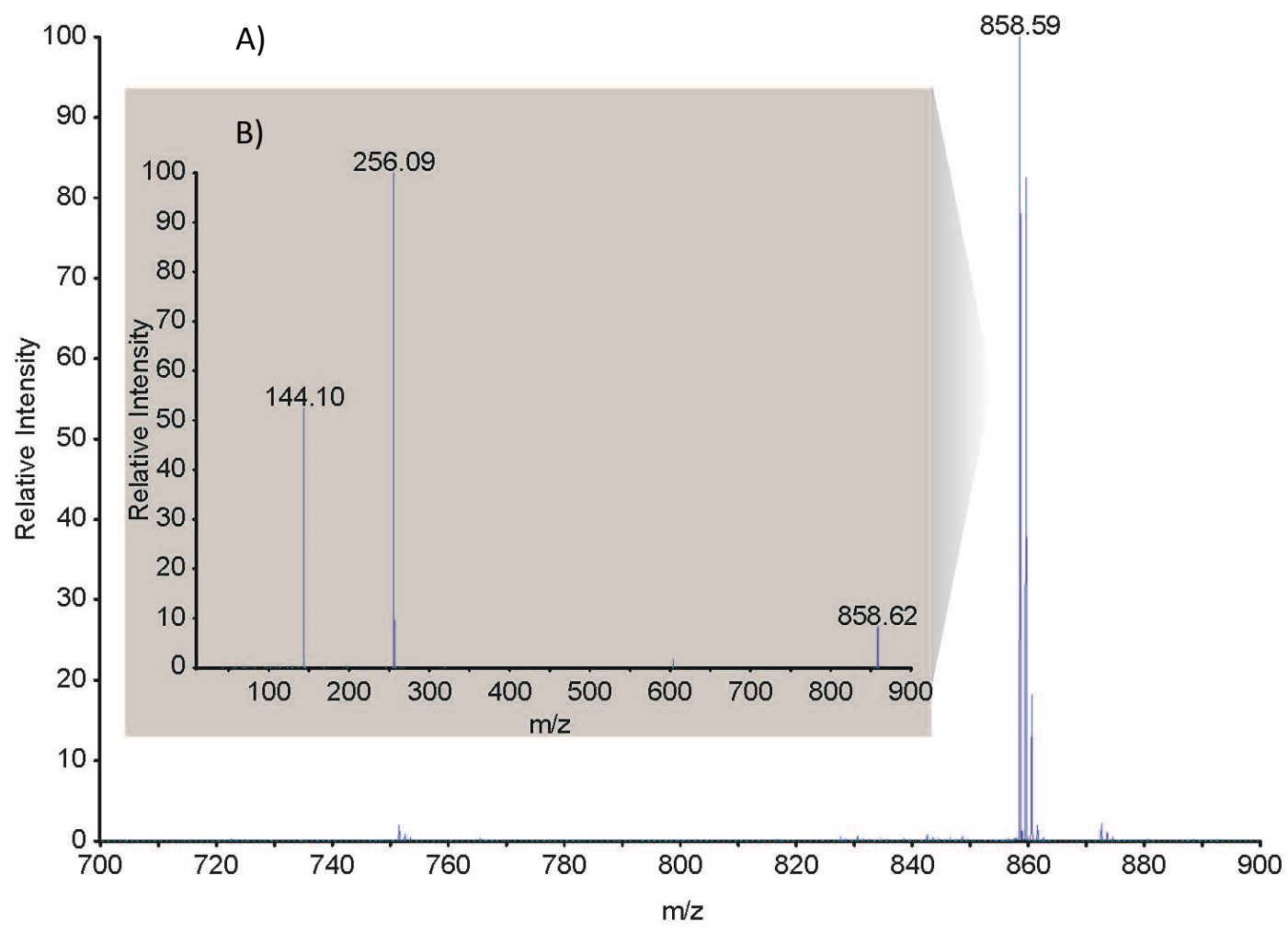

Figure 4.6: a) PS treated with diazomethane reveals five methyl groups ( $\mathrm{m} / \mathrm{z} 858.6)$. b) $\mathrm{MS}^{2}$ reveals two dominant fragmentation channels ( $\mathrm{m} / \mathrm{z} 256.1$ and 144.1 respectively)

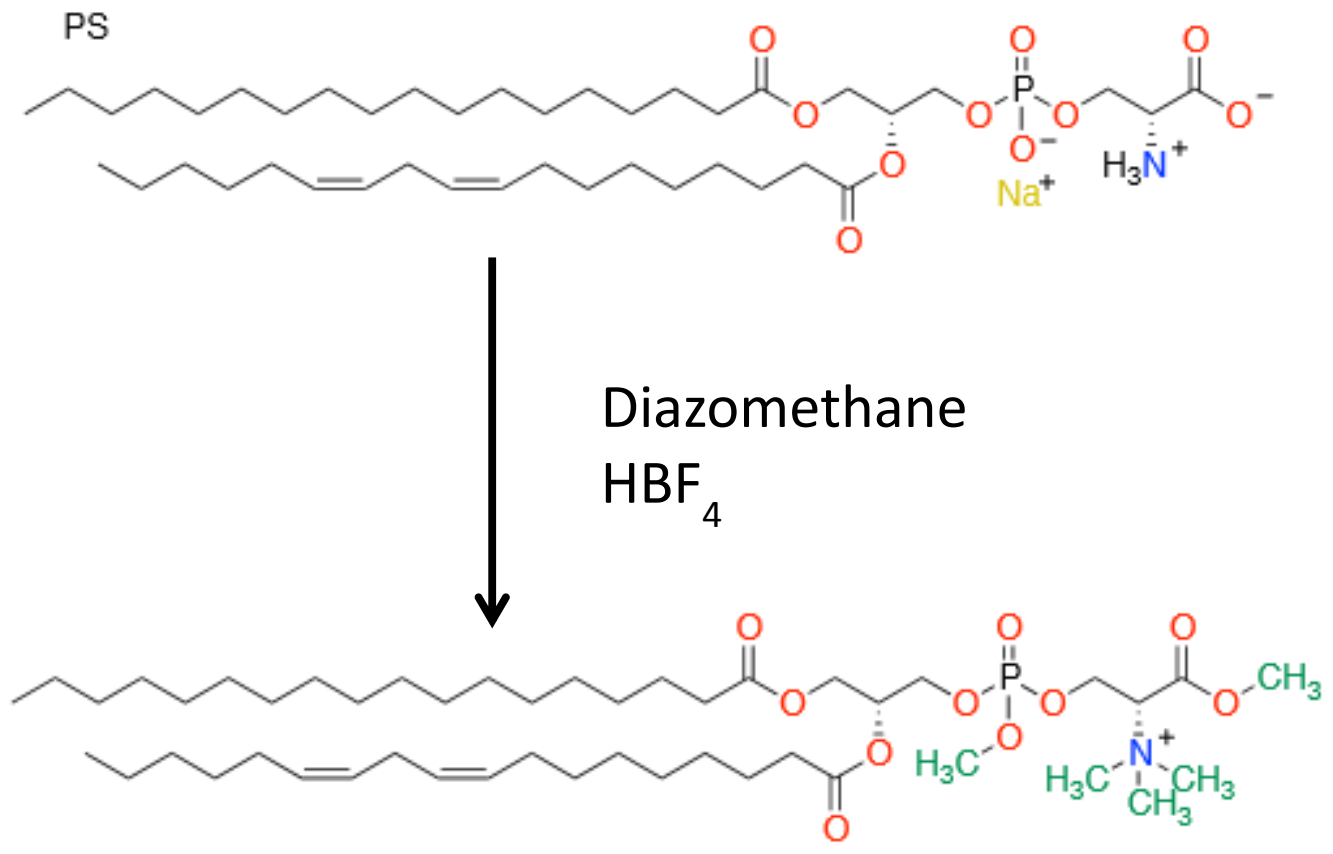

Figure 4.7: Pentamethylated $\mathrm{PS}(18: 0 / 18: 2(9 \mathrm{Z}, 12 \mathrm{Z}))$ via reaction with diazomethane and $\mathrm{HBF}_{4}$ 
To determine the reactivity of diazomethane with more frequently studied lipids, PC and SM standards were purchased and subjected to our methylating strategy. While other studies have shown the characteristic fragments formed in $\mathrm{MS}^{2}$ analysis of PC and SM lipids, ${ }^{8,57,58}$ here we observe the methylation of the phosphate group with no consequence to the parent molecule and its $\mathrm{MS}^{2}$ analysis (Figure 4.8 and Figure 4.9). The results of the treatment of each lipid with diazomethane and $\mathrm{HBF}_{4}$ in solution are summarized in Table 4.1.

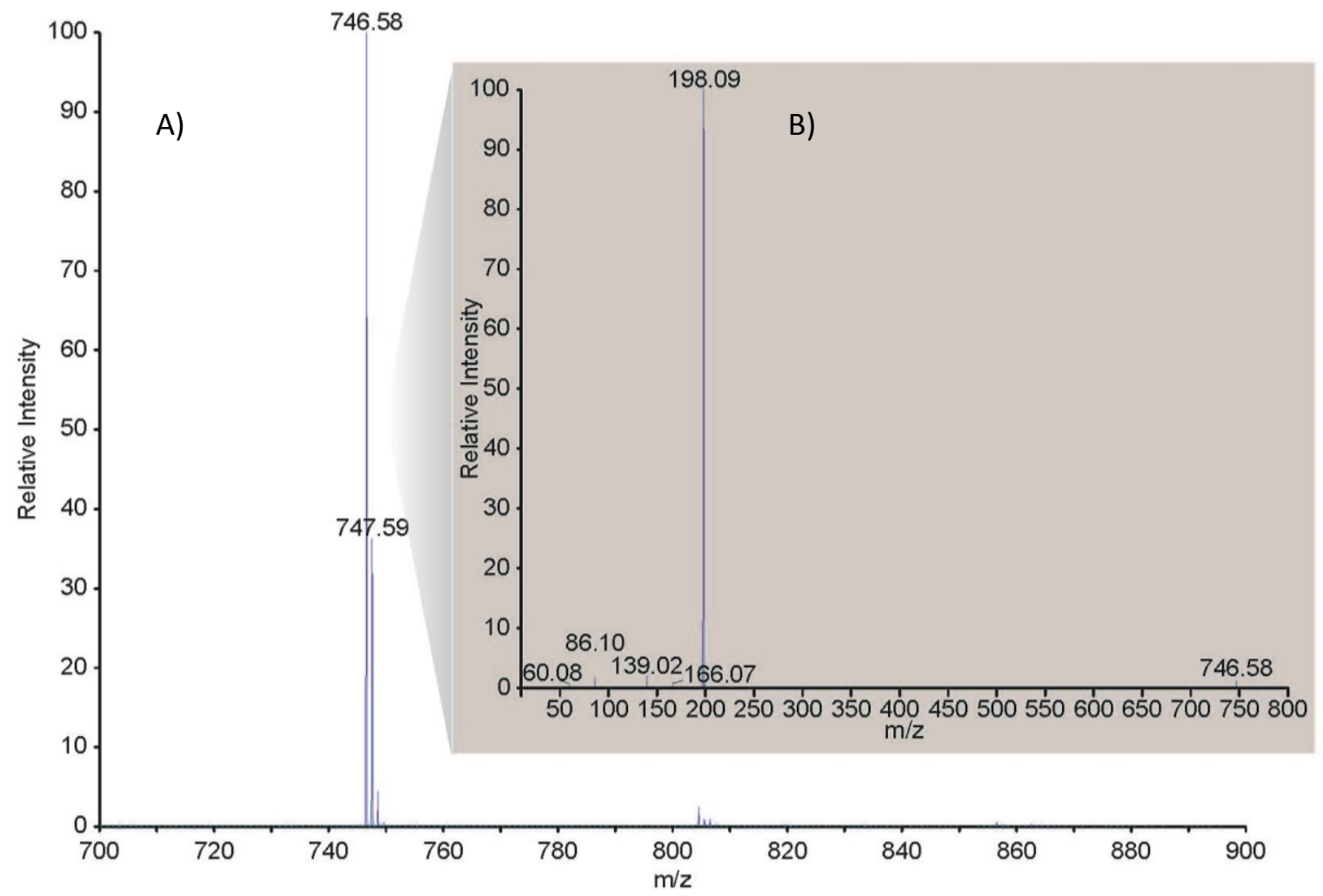

Figure 4.8: a) PC treated with diazomethane reveals addition of one methyl group $(\mathrm{m} / \mathrm{z} 746)$. b) Fragmentation of this lipid revealed one dominant fragmentation channel similar to the expected 184 Da head group with the additional methyl group (m/z 198.1) 


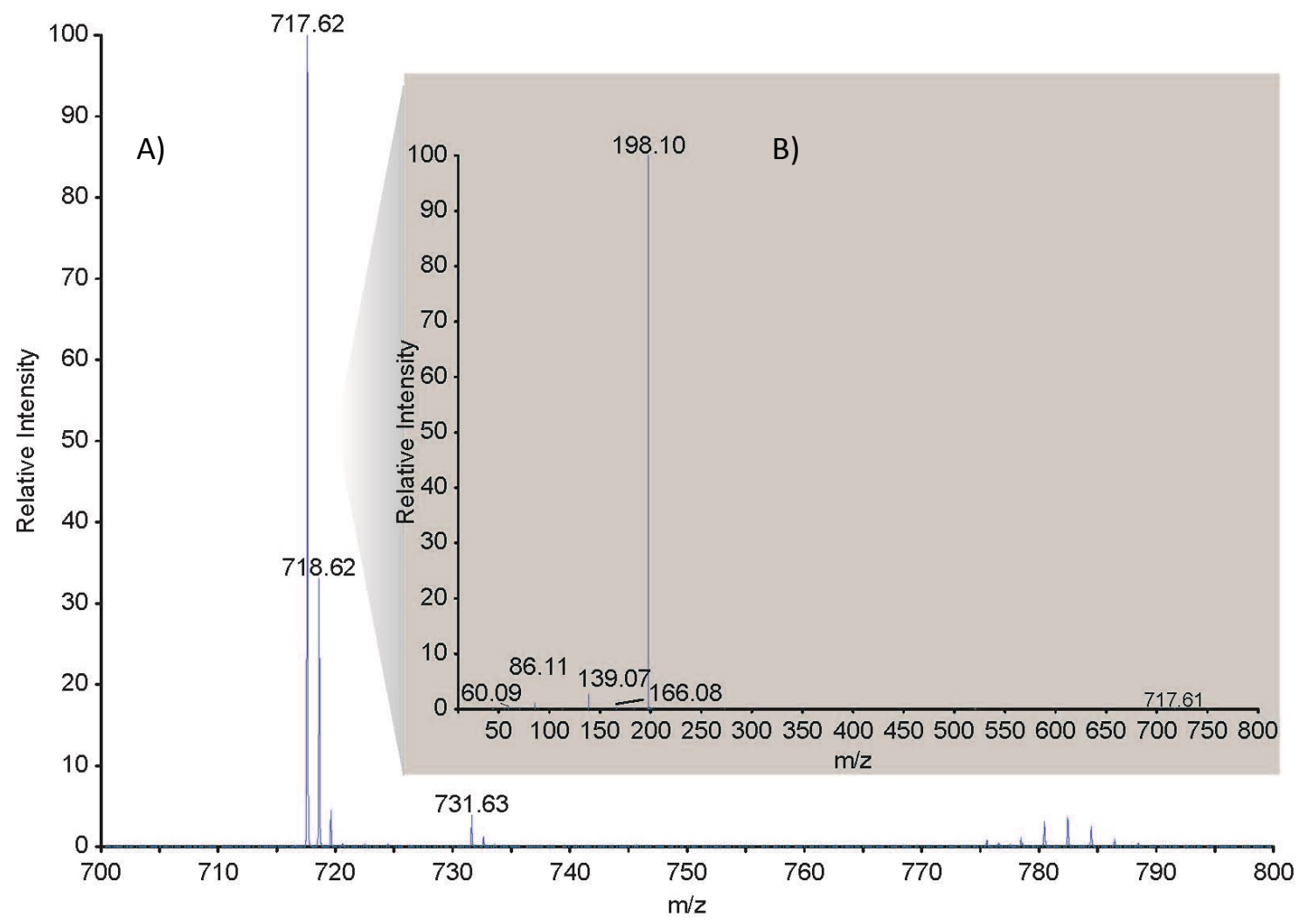

Figure 4.9: a) SM treated with diazomethane reveals addition of one methyl group $(\mathrm{m} / \mathrm{z}$ 717.6). b) Fragmentation of this lipid revealed one dominant fragmentation channel similar to the expected $184 \mathrm{Da}$ head group with the additional methyl group (m/z 198.1)

Table 4.1: Summary of results for diazomethane-modified synthetic lipids

\begin{tabular}{|c|c|c|c|c|c|c|c|}
\hline \multirow{2}{*}{ Lipid } & \multicolumn{2}{|c|}{ Unmodified } & $\begin{array}{c}\text { Modified } \\
\text { mass }\end{array}$ & $\Delta \mathrm{m}$ & \multicolumn{3}{c|}{ Methylation } \\
\cline { 2 - 8 } & $\mathrm{m} / \mathrm{z}$ & $\begin{array}{c}\text { neutral } \\
\text { mass }\end{array}$ & $\mathrm{m} / \mathrm{z}$ & $\mathrm{Da}$ & $\begin{array}{c}\% \\
\text { modified }\end{array}$ & $\begin{array}{c}\text { \# Me } \\
\text { groups }\end{array}$ & $\begin{array}{c}\text { \# fixed } \\
\text { charges }\end{array}$ \\
\hline$[\mathrm{PE}(16: 0 / 18: 1)]^{1+}$ & 718.5 & 717.5 & 774.6 & 56 & 100 & 4 & 1 \\
\hline$[\mathrm{PS}(18: 0 / 18: 2)]^{1+}$ & 809.5 & 810.5 & 858.5 & 70 & 100 & 5 & 1 \\
\hline$[\mathrm{PC}(18: 1 / 14: 0)]^{1+}$ & 759.6 & 760.6 & 774.6 & 14 & 100 & 1 & 1 \\
\hline$[\mathrm{SM}(\mathrm{d} 18: 1 / 16: 0)]^{1+}$ & 703.6 & 702.6 & 717.6 & 14 & 100 & 1 & 1 \\
\hline
\end{tabular}


Table 4.1 reveals that the negatively charged oxygen in the phosphate group was successfully methylated for all of the lipids that were tested using our strategy. Table 4.1 also indicates that primary amines and carboxylic acids are also successfully methylated. As with proteomic diazomethane studies (Chapter 3) these results indicate that the methylation efficiency of diazomethane is largely proportional to the pKa values of the lipid functional groups. Our results demonstrate that lipid functional groups with pKa values of approximately 11 or less are fully modified by diazomethane with the presence of acid. Functional groups with pKa values over 11 do not easily methylate within the timeframe of our experiment.

\subsubsection{MS sensitivity Increase Post TrEnDi}

Studies using equimolar amounts of unmodified lipid and modified lipid were conducted to verify that our derivatization strategy enhances ionization efficiency and sensitivity for individual lipids when analyzed by MS. For PE, there was nearly a $60 \%$ increase in sensitivity of the modified PE versus the unmodified PE (Figure 4.10a). The largest increase in sensitivity is visible in PS post diazomethane treatment. Here we see that an equimolar amount of unmodified PS produces a very weak and divided signal in comparison to the one dominating ion present post TrEnDi (Figure 4.10 b). A sensitivity test was also performed on PC and SM to demonstrate that there are no adverse effects with diazomethane treatment to the already strong signal of the unmodified PC or SM lipids. We do, however, observe a slight increase in 
sensitivity in PC through the neutralization of the negatively charged oxygen on the phosphate group as well as an increase in sensitivity for SM (Figure $4.10 \mathrm{c}$ and d).
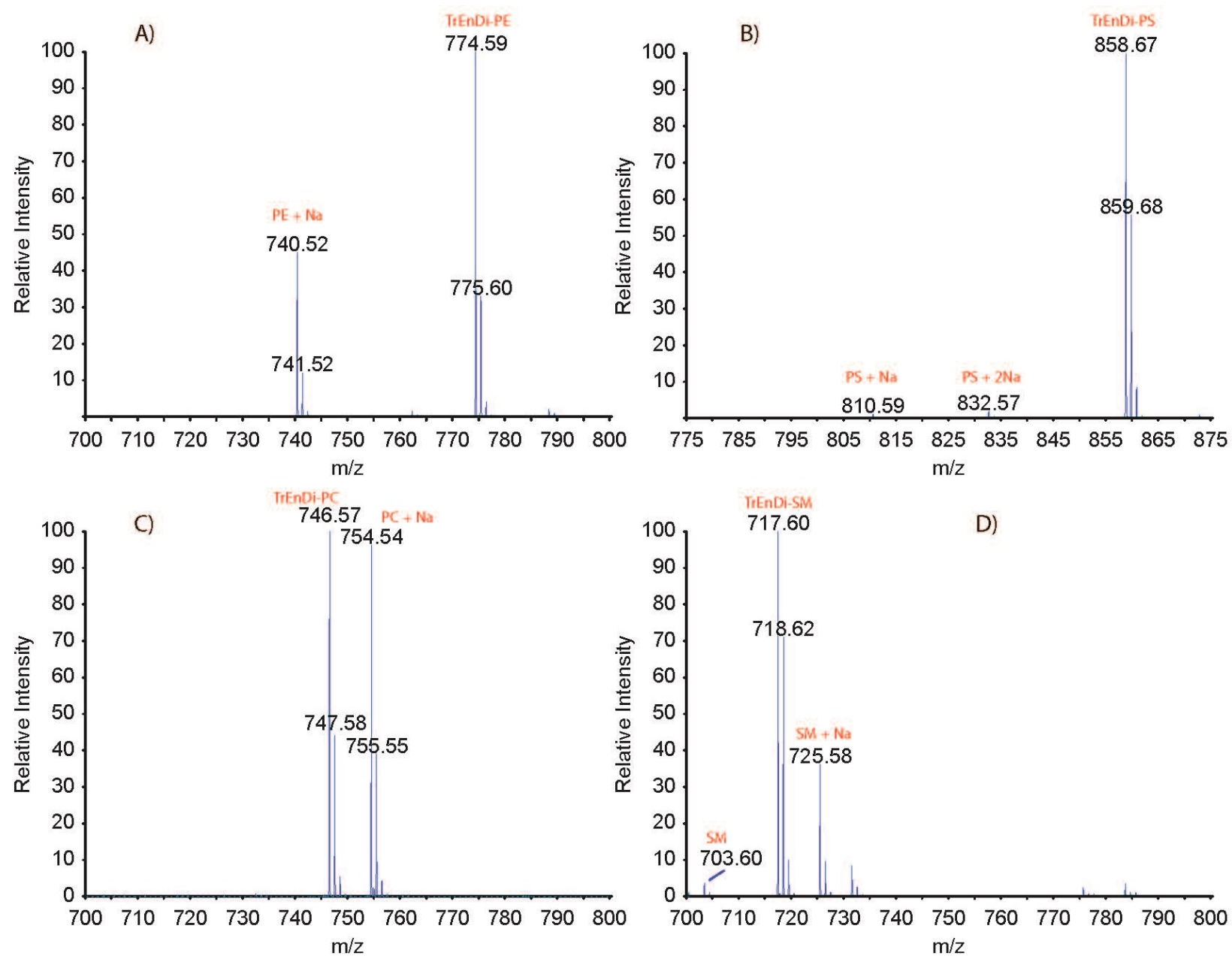

Figure 4.10: a) Equimolar amounts of modified and unmodified PE reveals an increase in sensitivity by nearly 60 percent. b) Equimolar amounts of modified and unmodified PS shows nearly $100 \%$ increase in sensitivity. c) Equimolar amounts of modified and unmodified PC show no significant increase in sensitivity. d) Equimolar amounts of modified and unmodified SM show an increase in sensitivity. 


\subsubsection{Multiple lipids tested simultaneously}

Our method demonstrated that lipids could be modified to a single stable product; however, it was unknown if there would be limitations to the methylation chemistry if a pool of several lipids were modified simultaneously. To test this, a solution containing PE, PS, SM and $\mathrm{PC}$ were acidified with the ethereal $\mathrm{HBF}_{4}$ solution and modified with the ethereal diazomethane solution to produce singly charged species. Our results demonstrated that the chemistry was equally effective on all lipids (Figure 4.11); PE, PS, SM and PC were all successfully methylated yielding MS signals of $\mathrm{m} / \mathrm{z} 774,858,717$ and 746 , respectively. $\mathrm{MS}^{2}$ on all 4 lipids confirmed the formation of their respective modified head groups with their characteristic mass shifts (Table 4.1). This experiment demonstrated that methylation of multiple different lipids was possible, opening the possibility of derivatizing more complex lipid mixtures.

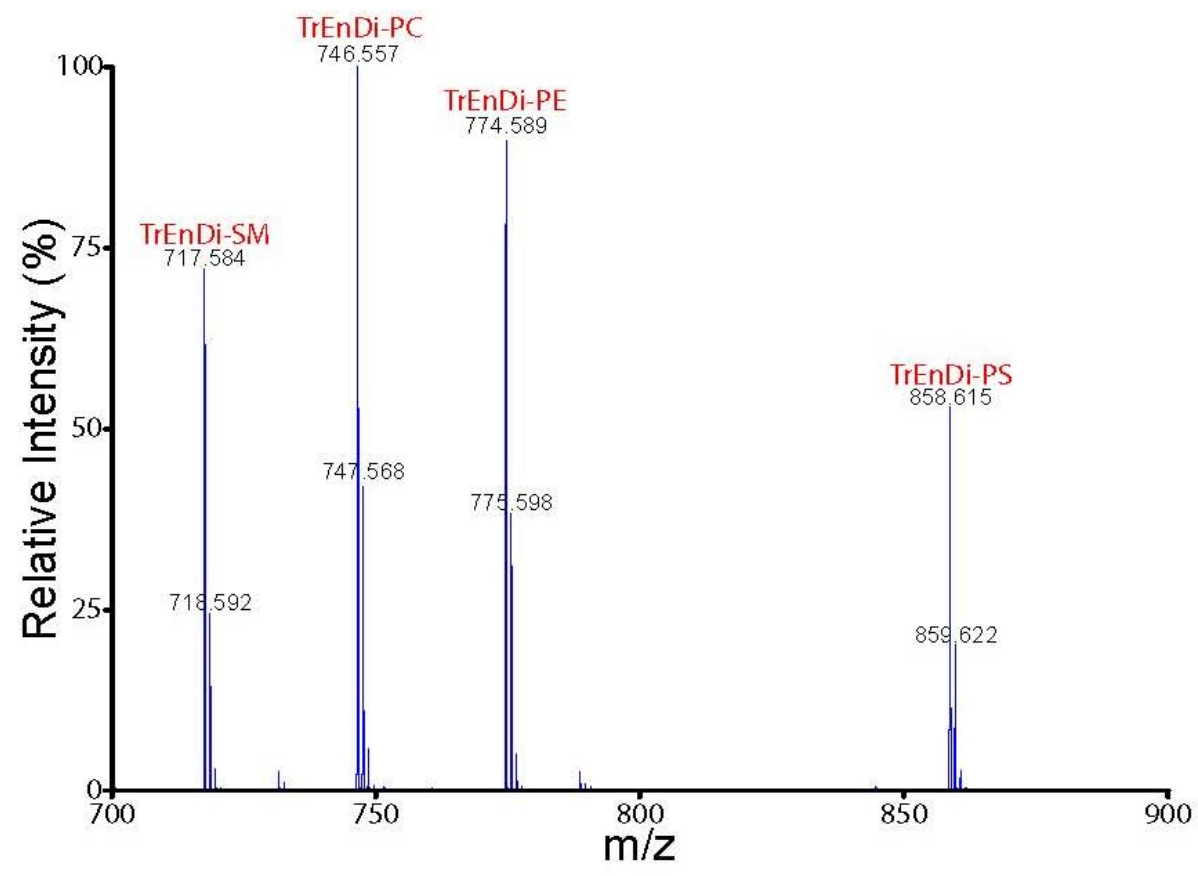

Figure 4.11: MS spectrum of mixture of TrEnDi-modified SM, PC, PE and PS 
In addition to this experiment, a sensitivity test was performed simultaneously on multiple lipid species. MS analysis in Figure 4.12 reveals the sensitivity increase gained from TrEnDi modified lipids over an equimolar amount of unmodified lipids that were spiked into the modified lipid combo solution. Unmodified PS is nearly non-existent with signals split between the protonated and sodiated parent ions.

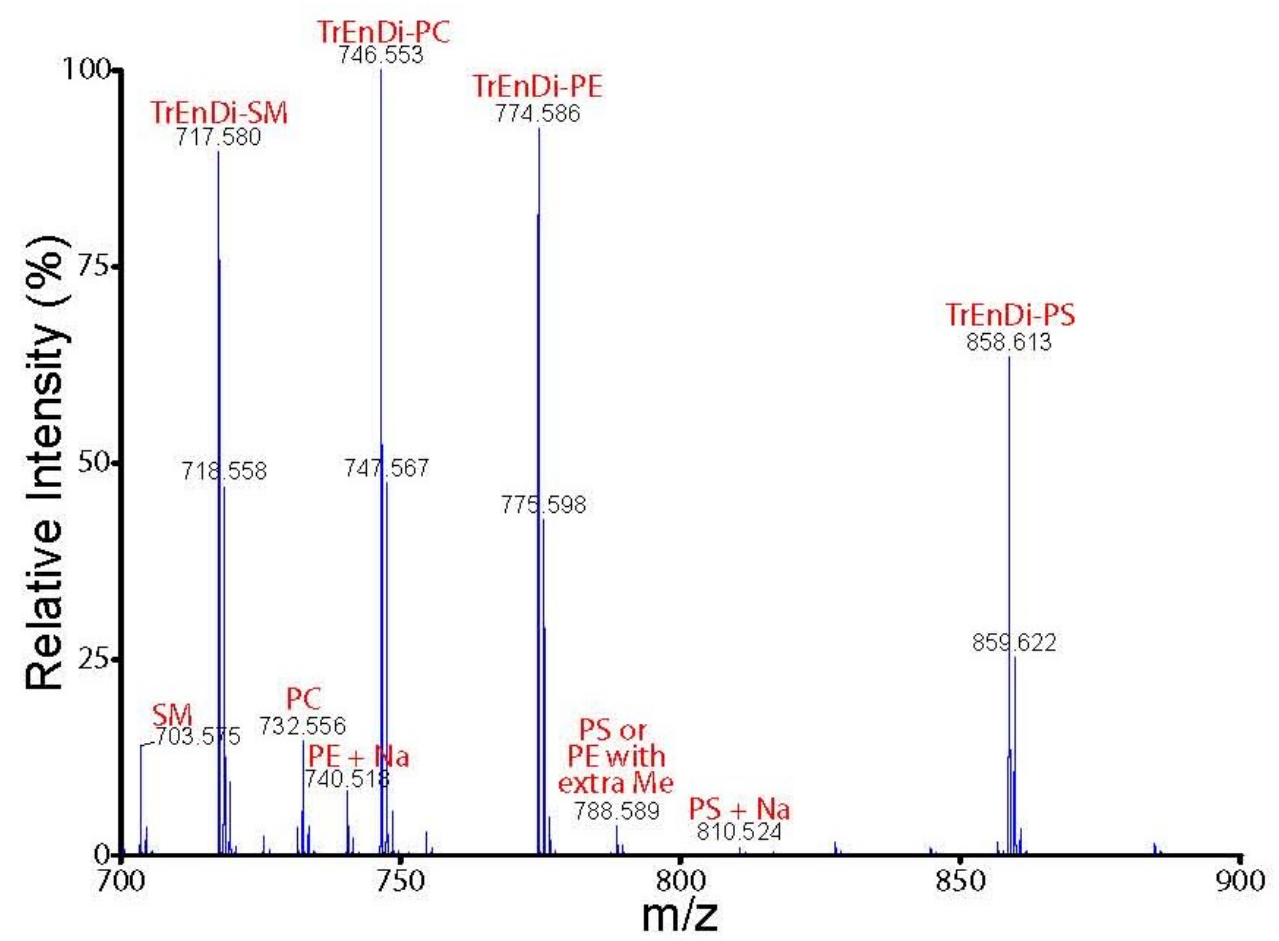

Figure 4.12: MS reveals the sensitivity increase gained from TrEnDi modified lipids over an equimolar amount of unmodified lipids 


\subsubsection{Precursor ion analyses sensitivity gain}

The ability of the precursor ion scanning mode on TrEnDi-modified lipids versus typical $M^{2}$ analysis of unmodified PC, SM, PE and PS were assessed (Table 4.2). The intensity of the signal in the neutral loss scanning mode for unmodified PE was relatively low and divided between the protonated and sodiated parent ions (Figure 4.13), while the neutral loss scanning mode for unmodified PS yielded very little observable signal (Figure 4.14). The precursor ion scan for $\mathrm{m} / \mathrm{z} 184$ yielded both unmodified PC and SM with a moderately intense signal (Figure 4.15). However, the precursor ion scanning method was able to detect all four TrEnDi-modified lipids with signal intensities significantly greater than those observed in the unmodified lipid analyses (Figure 4.16 and Figure 4.17). The greatest increase in sensitivity was found for PS with a precursor scan of $\mathrm{m} / \mathrm{z} 144$ with CE of 60eV (Figure 4.18).

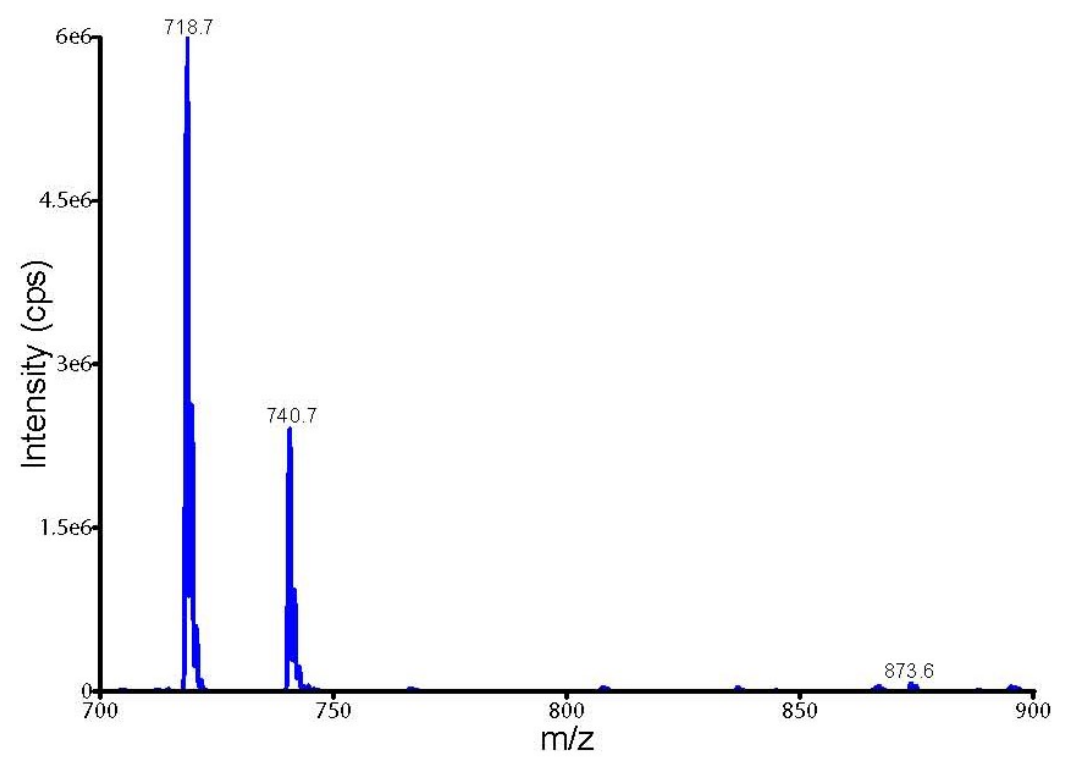

Figure 4.13: Divided signal intensities of the neutral loss scan for PE (-m/z 141) 


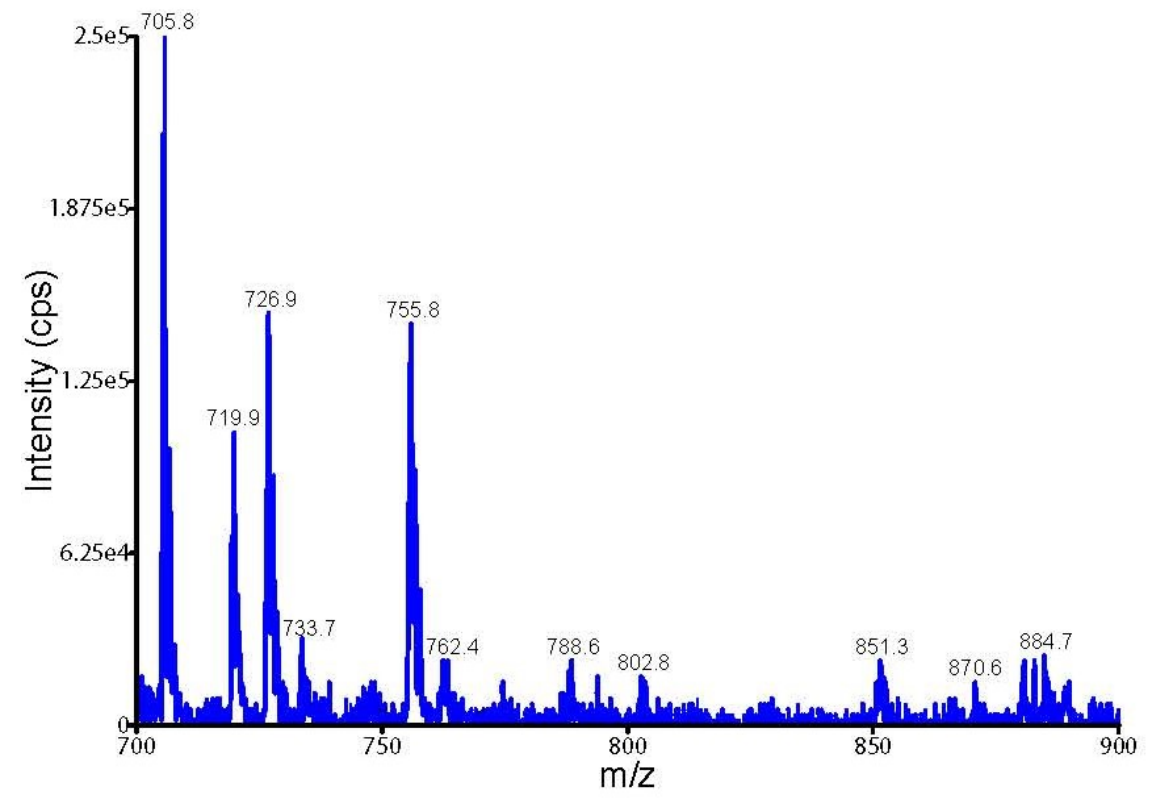

Figure 4.14: Very weak signal intensity in the neutral loss scan for PS (-m/z 185)

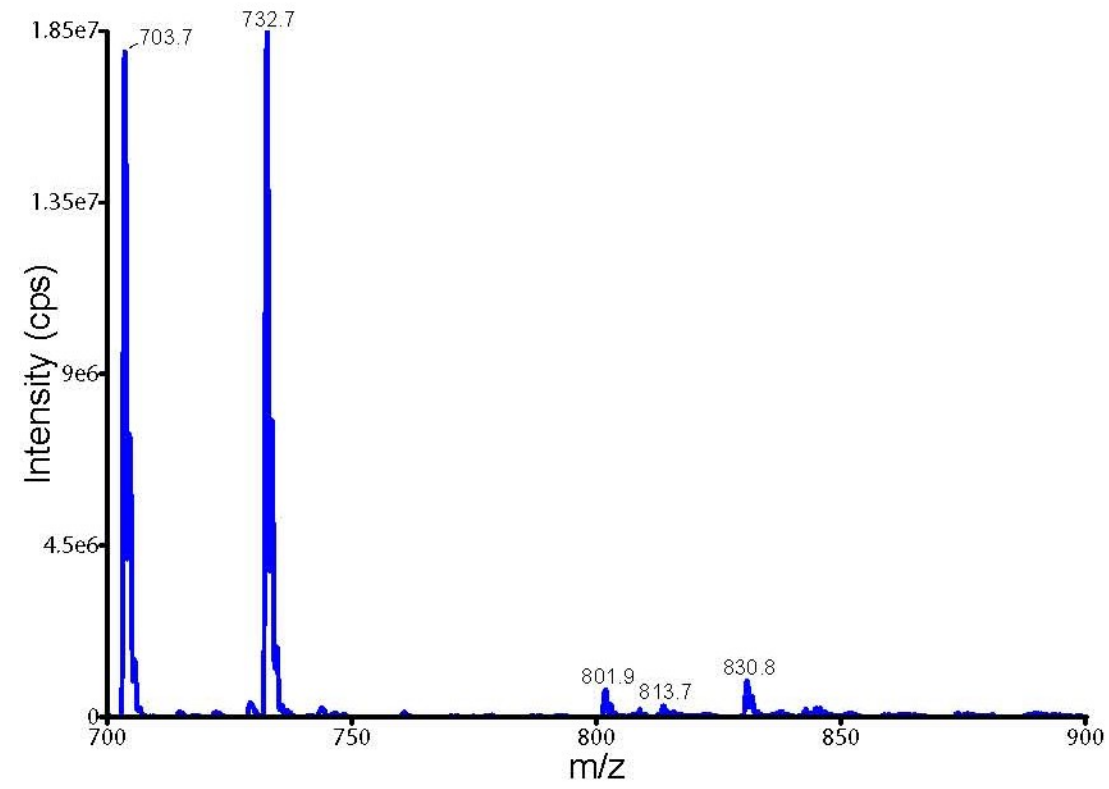

Figure 4.15: Relatively strong signal intensities for the precursor ion scan $(\mathrm{m} / \mathrm{z} 184)$ for PC $(\mathrm{m} / \mathrm{z} 732.7)$ and SM $(\mathrm{m} / \mathrm{z}$ 703.7) 


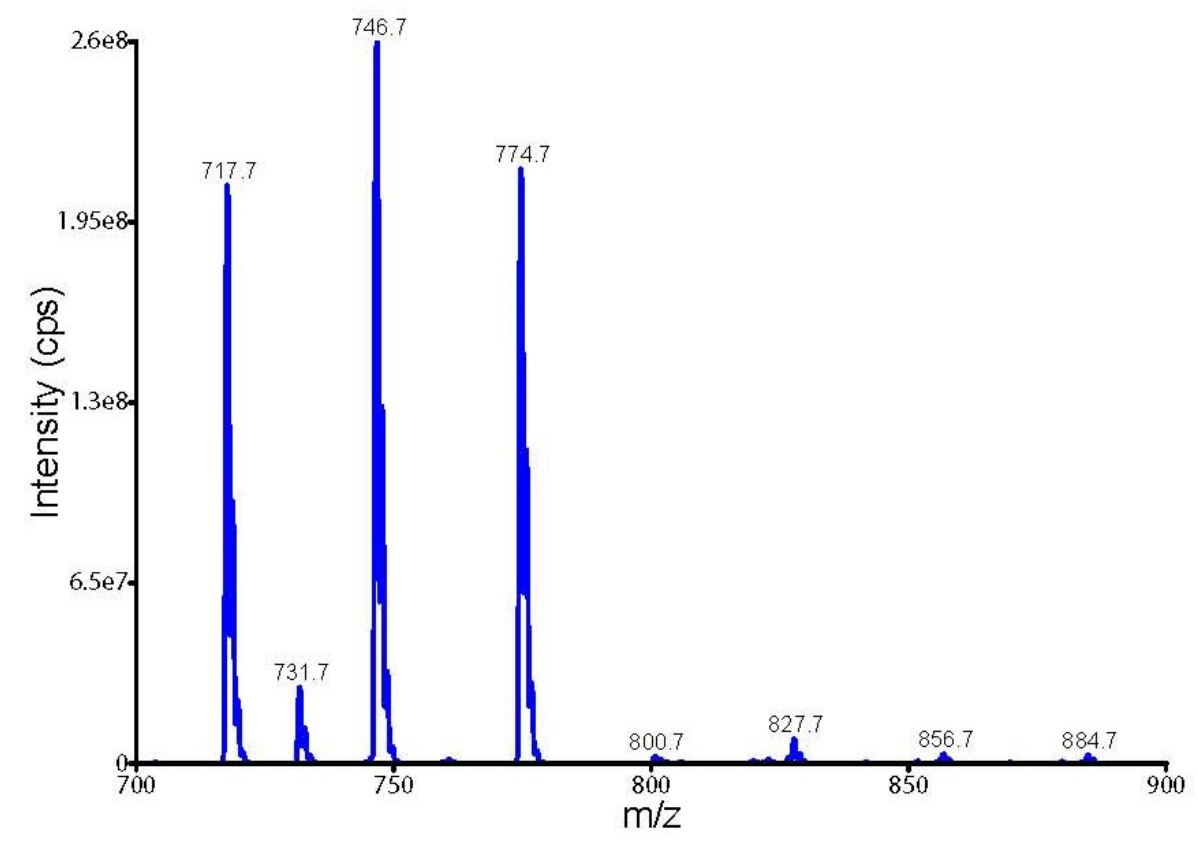

Figure 4.16: Significantly stronger signal intensities for the precursor ion scan $(\mathrm{m} / \mathrm{z} 198)$ for TrEnDi-modified SM (m/z 717.7), PC (m/z 746.7) and PE (m/z 774.7)

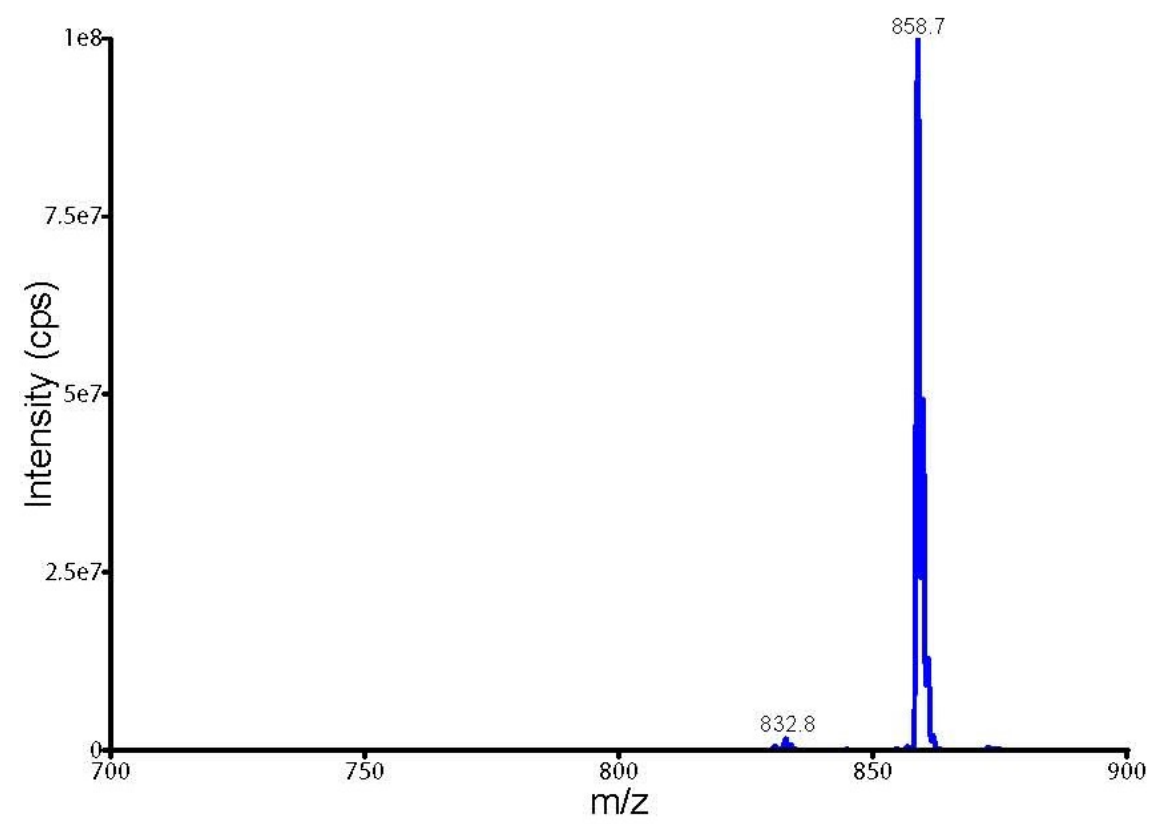

Figure 4.17: Significantly stronger signal intensity in the precursor ion scan $(\mathrm{m} / \mathrm{z} 256)$ for TrEnDi-modified PS (m/z 858.7) 


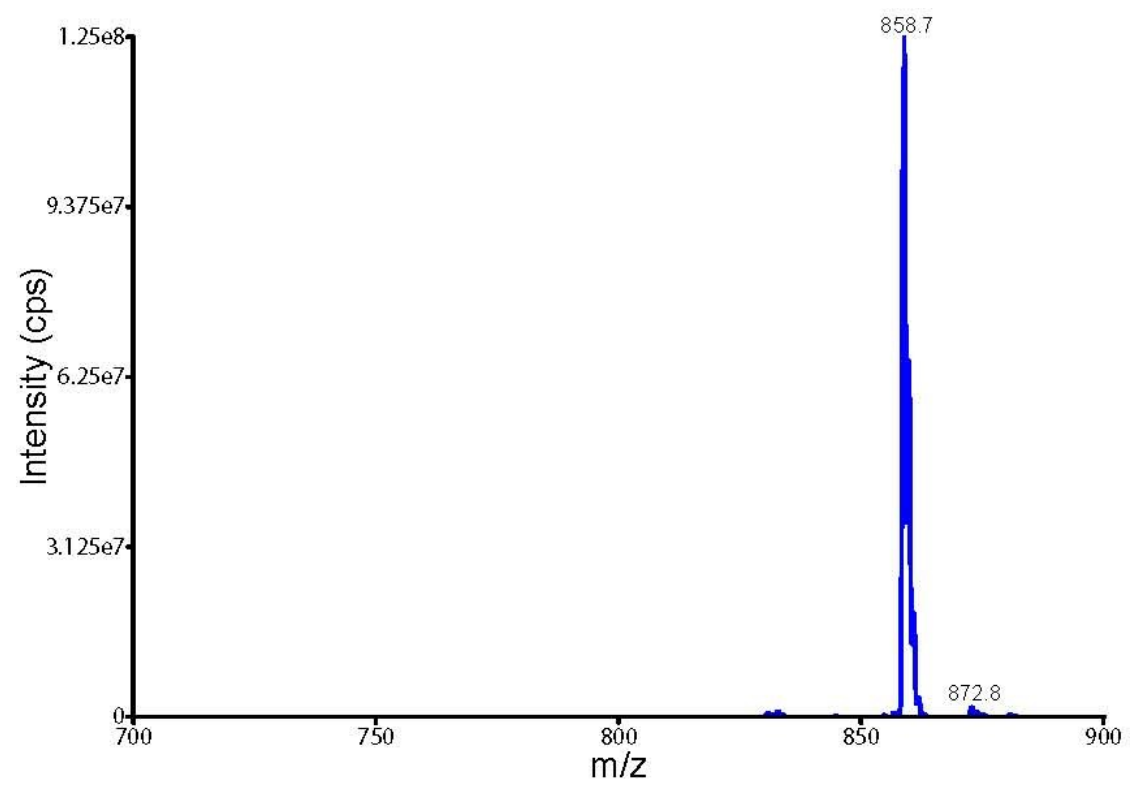

Figure 4.18: The strongest signal intensity for the precursor ion scan $(\mathrm{m} / \mathrm{z} 144)$ with CE of 60eV for TrEnDi-modified PS ( $\mathrm{m} / \mathrm{z}$ 858.7)

Our results demonstrate that TrEnDi-modified lipids produce significantly stronger signal intensities when performing $\mathrm{MS}^{2}$ analysis, with an increase in signal of up to 4 orders of magnitude.

Table 4.2: Summary of interesting transitions for unmodified and TrEnDi-modified lipids

\begin{tabular}{|c|c|c|c|c|}
\hline \multirow{2}{*}{ Lipids } & \multicolumn{2}{|c|}{ TrEnDi-modified } & \multicolumn{2}{c|}{ Unmodified } \\
\cline { 2 - 5 } & $\mathrm{m} / \mathrm{z}$ parent & $\mathrm{m} / \mathrm{z}$ fragment & $\mathrm{m} / \mathrm{z}$ parent & $\mathrm{m} / \mathrm{z}$ fragment \\
\hline$[\mathrm{PE}(16: 0 / 18: 1)]^{1+}$ & 774.5 & 198.1 & 718.5 & -141 \\
\hline$[\mathrm{PS}(18: 0 / 18: 2)]^{1+}$ & 858.5 & 256 & 788.5 & -185 \\
\cline { 2 - 5 } & & 144 & & 184.1 \\
\hline$[\mathrm{PC}(18: 1 / 14: 0)]^{1+}$ & 746.5 & 198.1 & 732.5 & 184.1 \\
\hline$[\mathrm{SM}(\mathrm{d} 18: 1 / 16: 0)]^{1+}$ & 717.5 & 198.1 & 703.5 & \\
\hline
\end{tabular}




\subsection{Conclusion}

The trimethylation enhancement using diazomethane (TrEnDi) technique has been successfully developed on various lipids. Complete methylation has also been shown to occur on functional groups with pKa values lower than 11 and produce quaternary ammonium groups on PE and PS primary amines. Phosphate moieties and carboxylic acids were also shown to be methylated using this chemistry (neutralizing the negative charge); however, secondary alcohol groups were less affected.

TrEnDi modifies lipids such that they contain fixed, permanent positive charges, increasing the sensitivity of ESI MS analysis by driving the charge state of each lipid to a single value and opening the possibility of using aprotic solvents. The derivatization chemistry is predictable but equally important is that the fragmentation behaviour of TrEnDi-modified lipids is also predictable. TrEnDi-modified lipids demonstrate a propensity to fragment and form the fully modified head group ions. The fully modified head group fragment was the most intense fragment for PC, PE and SM lipids, while higher collision energies produced an alternate fragment for PS with even greater signal intensity, revealing how this chemistry is able to enhance the sensitivity of precursor ion scanning analyses. The proof of this sensitivity gain was observed in the comparison analysis of equimolar amounts of unmodified and TrEnDimodified lipids using conventional lipidomics methods. TrEnDi places a fixed positive charge on lipids and induces anticipated head group fragmentation behaviour, permitting highly sensitive 
precursor ion scanning experiments and providing a mechanism to observe and quantify elusive lipids. 


\subsection{Other projects and future works}

\subsection{Protein Research}

The focus of this study was to determine the effectiveness of TrEnDi on complex protein digests. Horse heart myoglobin was chosen as a suitable candidate as it is a relatively small protein of 153 amino acids in length and in the $17 \mathrm{kDa}$ mass range (the sequence is shown in Figure 5.1).

$\begin{array}{rlllll}1 & \text { GLSDGEWQQV } & \text { LNVWGKVEAD } & \text { IAGHGQEVLI } & \text { RLFTGHPETL } & \text { EKFDKFKHLK } \\ 51 & \text { TEAEMKASED } & \text { LKKHGTVVLT } & \text { ALGGILKKKG } & \text { HHEAELKPLA } & \text { QSHATKHKIP } \\ 101 & \text { IKYLEFISDA } & \text { IIHVLHSKHP } & \text { GDFGADAQGA } & \text { MTKALELFRN } & \text { DIAAKYKELG } \\ 151 & \text { FQG } & & & & \end{array}$

Figure 5.1: Amino acid sequence of horse heart myoglobin

Myoglobin was tryptically digested as previously described in section 2.6 , and subjected to an IDA experiment to determine the total sequence coverage of the unmodified protein. Analysis of the IDA experiment indicated a positive match for 7 peptides listed in Table 5.1.

Table 5.1: List of peptides observed pre-modification

\begin{tabular}{|l|c|}
\hline Peptide & m/z Da \\
\hline ELGFQG & 650.3 \\
\hline ALELFR & 748.5 \\
\hline YKELGFQG & 471.3 \\
\hline LFTGHPETLEK & 424.6 \\
\hline ALELFRNDIAAK & 454.3 \\
\hline HGTVVLTALGGILK & 460.3 \\
\hline VEADIAGHGQEVLIR & 536.3 \\
\hline
\end{tabular}


These peptides are equivalent to $39.2 \%$ sequence coverage of the entire protein. The hypothesis of this experiment was that the tryptically digested myoglobin subjected to oncolumn TrEnDi would provide greater sequence coverage and produce higher intensity peaks than that observed from the unmodified myoglobin. The mass spectrum of the tryptically digested myoglobin following treatment with diazomethane is shown in Figure 5.2. Figure 5.2 and Table 5.2 reveals only three fully modified peptides, including $[\text { ALELFR }]^{2+},\left[\right.$ LFTGHPETLEK] ${ }^{3+}$ and [VEADIAGHGQEVLIR] ${ }^{3+}$ ions at $\mathrm{m} / \mathrm{z} 395.8,462.0$ and 559.7 respectively, were observed. A total mass shift of +42 Da from the unmodified $[A L E L F R]^{2+},+112$ Da from the unmodified [LFTGHPETLEK] $^{3+}$ and +70 Da from the unmodified [VEADIAGHGQEVLIR] ${ }^{3+}$ was observed, indicating that the peptides had been methylated three, eight and five times respectively.

Table 5.2: List of peptides observed post-TrEnDi modification

\begin{tabular}{|l|c|}
\hline Peptide & m/z Da \\
\hline ALELFR & 395.8 \\
\hline LFTGHPETLEK & 462.0 \\
\hline VEADIAGHGQEVLIR & 559.7 \\
\hline
\end{tabular}




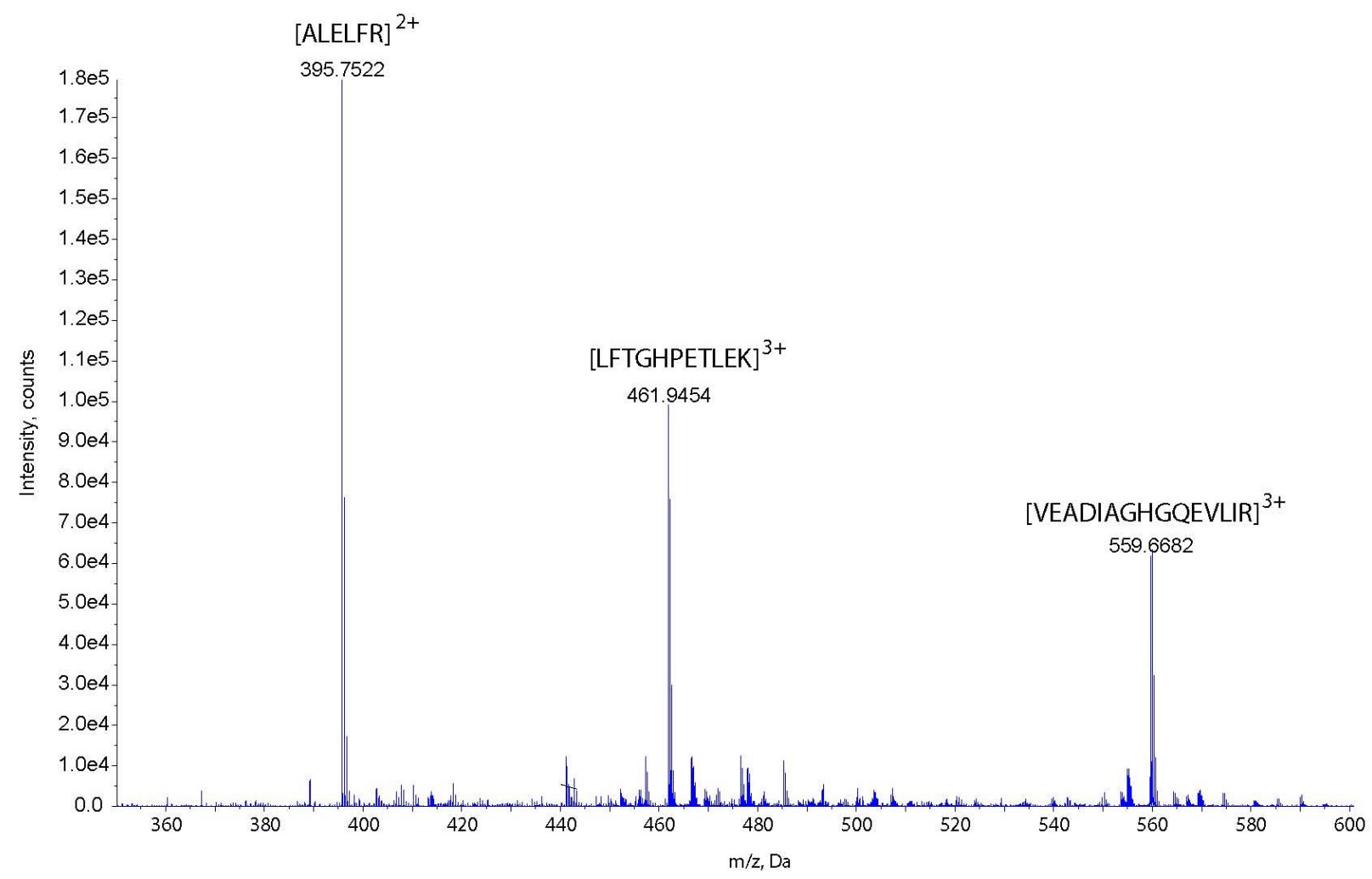

Figure 5.2: MS analysis of myoglobin confirms three peptides fully modified

Figure 5.3, Figure 5.4 and Figure 5.5 confirm that the peptides were fully methylated as predicted with successful base hydrolysis of the methyl esters and formation of the dominant $a_{2}$ fragment ion. Tables 5.3 through 5.5 highlight the total sequence coverage obtained post-

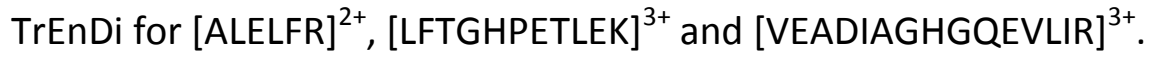




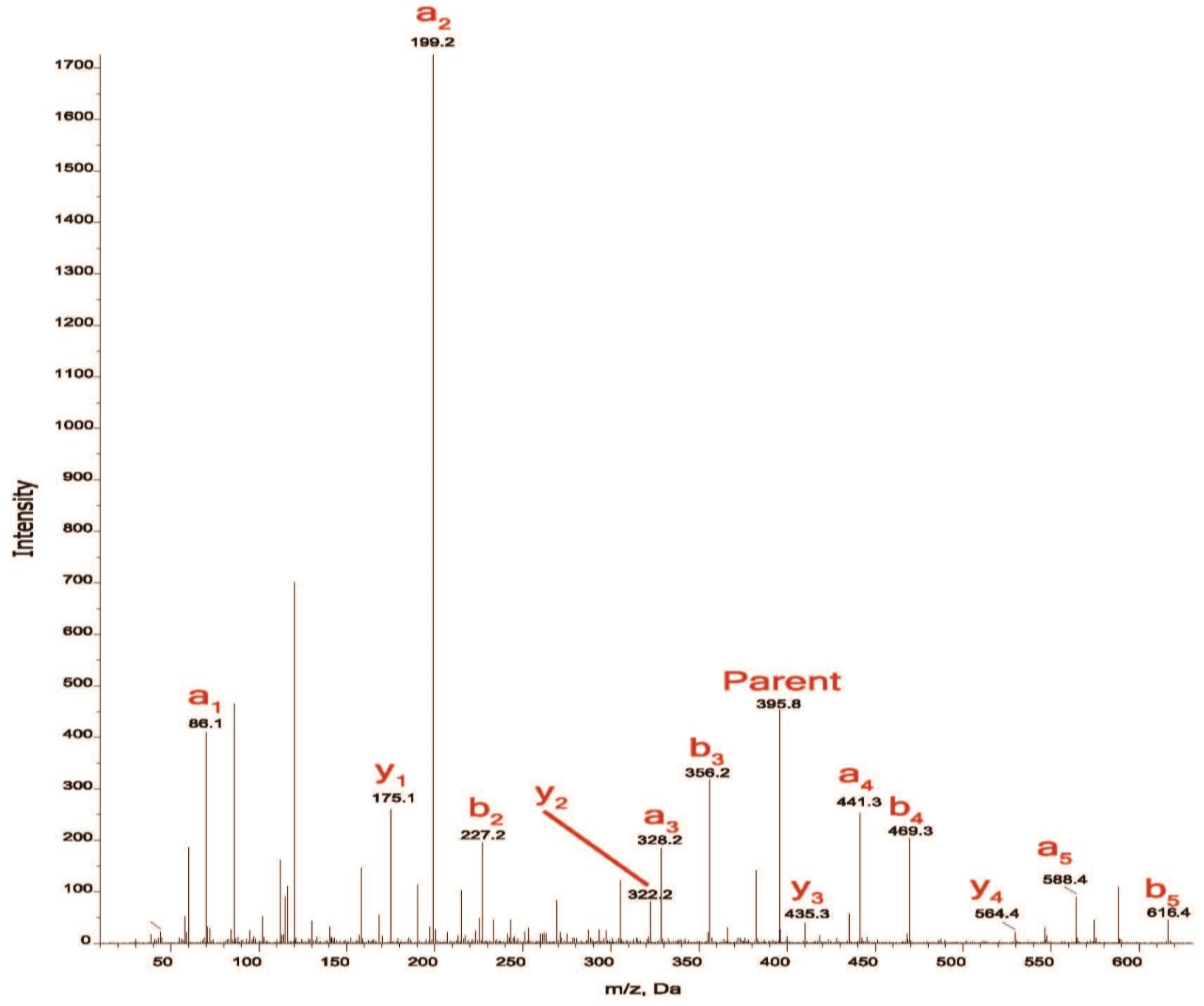

Figure 5.3: $\mathrm{MS}^{2}$ analysis of TrEnDi modified ALELFR.

Table 5.3: Predicted $a, b$ and $y$ ions versus observed ions highlighted in yellow in the $\mathrm{MS}^{2}$ analysis of ALELFR

\begin{tabular}{ccccc} 
& \multicolumn{3}{c}{ Fragment ions } \\
Location & $\mathrm{a}$ & $\mathrm{b}$ & $\mathrm{Y}$ \\
\hline 1 & 86.09717 & 114.0921 & 175.1196 \\
2 & 199.1812 & 227.1761 & 322.188 \\
3 & 328.2238 & 356.2187 & 435.2721 \\
4 & 441.3079 & 469.3028 & 564.3146 \\
\hline 5 & 588.3763 & 616.3712 & 677.3987 \\
6 & 372.7426 & 386.7401 & 748.4358
\end{tabular}




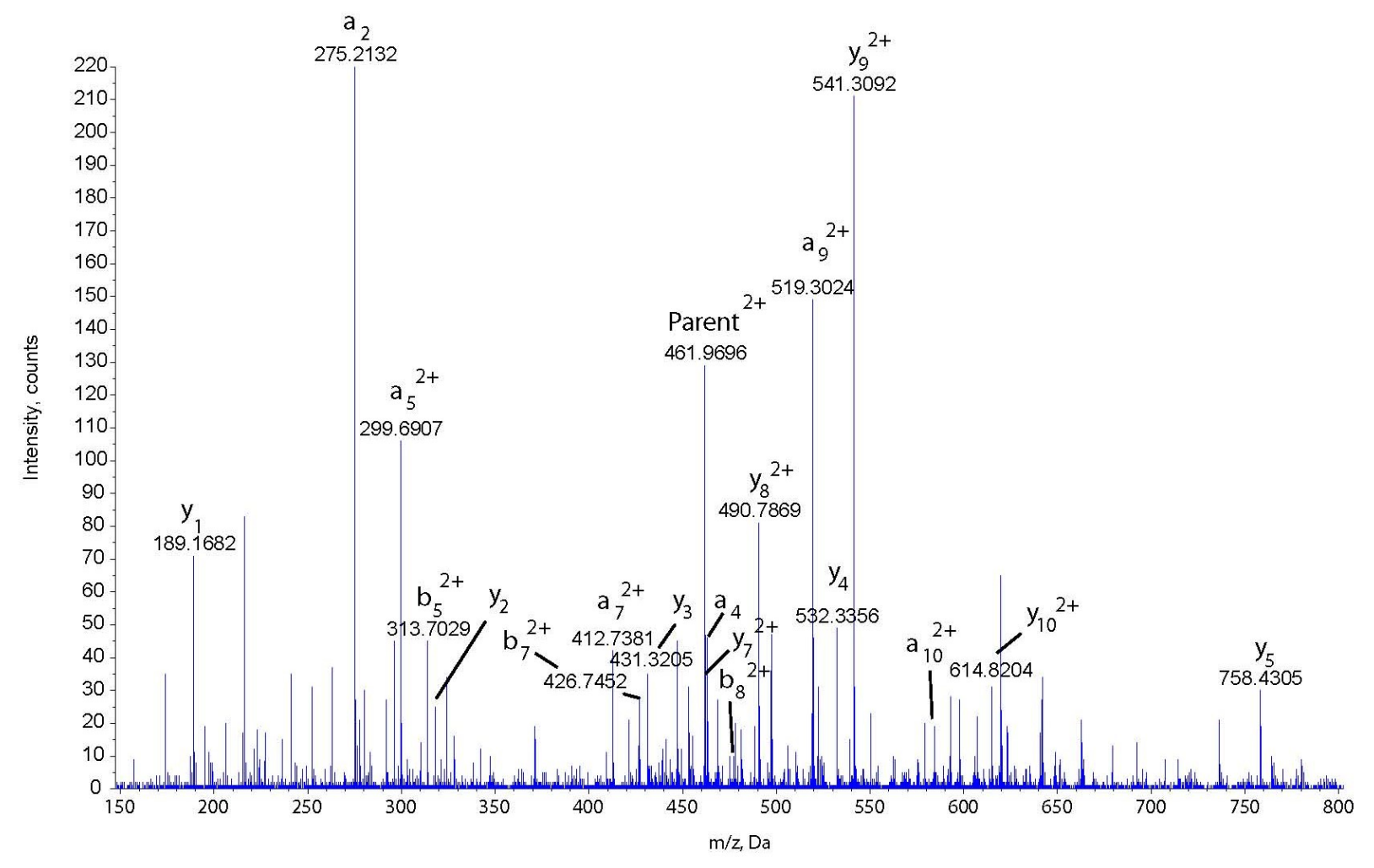

Figure 5.4: $\mathrm{MS}^{2}$ analysis of TrEnDi modified LFTGHPETLEK

Table 5.4: Predicted $a, b$ and $y$ ions versus observed ions highlighted in yellow in the $\mathrm{MS}^{2}$ analysis of LFTGHPETLEK

\begin{tabular}{cccc} 
& \multicolumn{3}{c}{ Fragment ions } \\
location & $a$ & $b$ & $Y$ \\
1 & 128.1441 & 156.139 & 189.1606 \\
2 & 275.2125 & 303.2074 & 318.2031 \\
3 & 376.2602 & 404.2551 & 431.2872 \\
4 & 433.2817 & 461.2766 & 532.3349 \\
5 & 299.6899 & 313.6874 & 661.3775 \\
6 & 348.2163 & 362.2138 & 758.4302 \\
7 & 412.7376 & 426.7351 & 462.2642 \\
8 & 463.2614 & 477.2589 & 490.7749 \\
9 & 519.8035 & 533.8009 & 541.2988 \\
10 & 584.3248 & 598.3222 & 614.833 \\
\hline 11 & 446.5998 & 455.9315 & 671.375
\end{tabular}




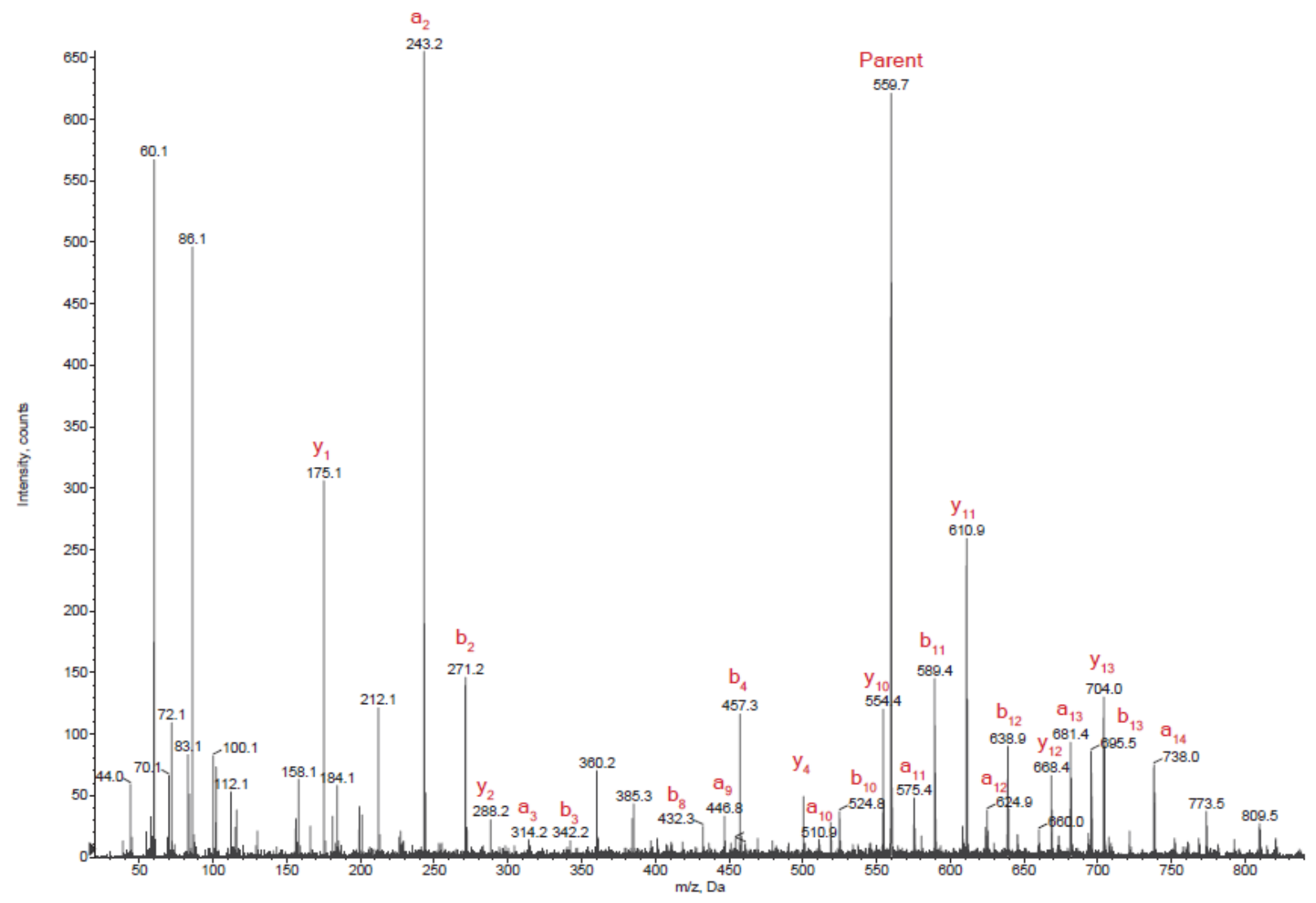

Figure 5.5: $\mathrm{MS}^{2}$ analysis of TrEnDi modified VEADIAGHGQEVLIR.

Table 5.5: Predicted $a, b$ and y ions versus observed ions highlighted in yellow in the $\mathrm{MS}^{2}$ analysis of VEADIAGHGQEVLIR

$\begin{array}{ccccc}\text { Location } & a & b & Y \\ 1 & 114.1285 & 142.1234 & 175.1196 \\ 2 & 243.1711 & 271.166 & 288.2036 \\ 3 & 314.2082 & 342.2031 & 401.2877 \\ 4 & 429.2351 & 457.23 & 500.3561 \\ 5 & 542.3192 & 570.3141 & 629.3987 \\ 6 & 613.3563 & 641.3512 & 757.4573 \\ 7 & 670.3777 & 698.3727 & 814.4787 \\ 8 & 418.238 & 432.2354 & 490.2885 \\ 9 & 446.7487 & 460.7461 & 518.7992 \\ 10 & 510.778 & 524.7754 & 554.3177 \\ 11 & 575.2993 & 589.2967 & 610.8598 \\ 12 & 624.8335 & 638.8309 & 668.3732 \\ 13 & 681.3755 & 695.373 & 703.8918 \\ 14 & 737.9175 & 751.915 & 768.4131 \\ 15 & 544.3147 & 553.6463 & 817.9473\end{array}$


It remains unclear as to why the other peptides were not successfully observed postTrEnDi modification. There is the possibility that the other peptides had a lower binding affinity to the $\mathrm{SCX}$ resin. Another more likely theory that remains to be tested for future studies, is that post-TrEnDi modified peptides can become highly charged (some peptides were predicted to have 6 fixed positive charges). These highly charged species would become more hydrophilic, making the desalting step challenging as the fully modified peptides would likely have difficulties binding to the $\mathrm{C} 18$ resin during the desalting procedure. In an attempt to solve this problem, future full protein on-column TrEnDi studies should therefore be tested with triethylammonium acetate as the elution solution in order to increase hydrophobicity (section 5.3). This volatile salt can then be evaporated and the TrEnDi modified protein can be dissolved in a more suitable solvent for MS analysis.

\subsection{Phosphopeptides and ${ }^{18} 0$ labelling}

Phosphopeptide work was performed on-column following the general procedures found in section 2.3. Here the main focus was on a synthesized peptide FLEEpSK with a phosphorylated serine. The goal of this experiment was to determine the effects TrEnDi would have on a phosphorylated peptide. The unmodified FLEEPSK peptide was found to be primarily a doubly charged ion with a mass-to-charge ratio of $416.71 \mathrm{Da}$ as shown in Figure 5.6. $\mathrm{MS}^{2}$ data of the unmodified FLEEPSK revealed some characteristic fragment ions including the neutral loss of the serine phosphate group in the $y$ ion series (see Figure 5.7). 


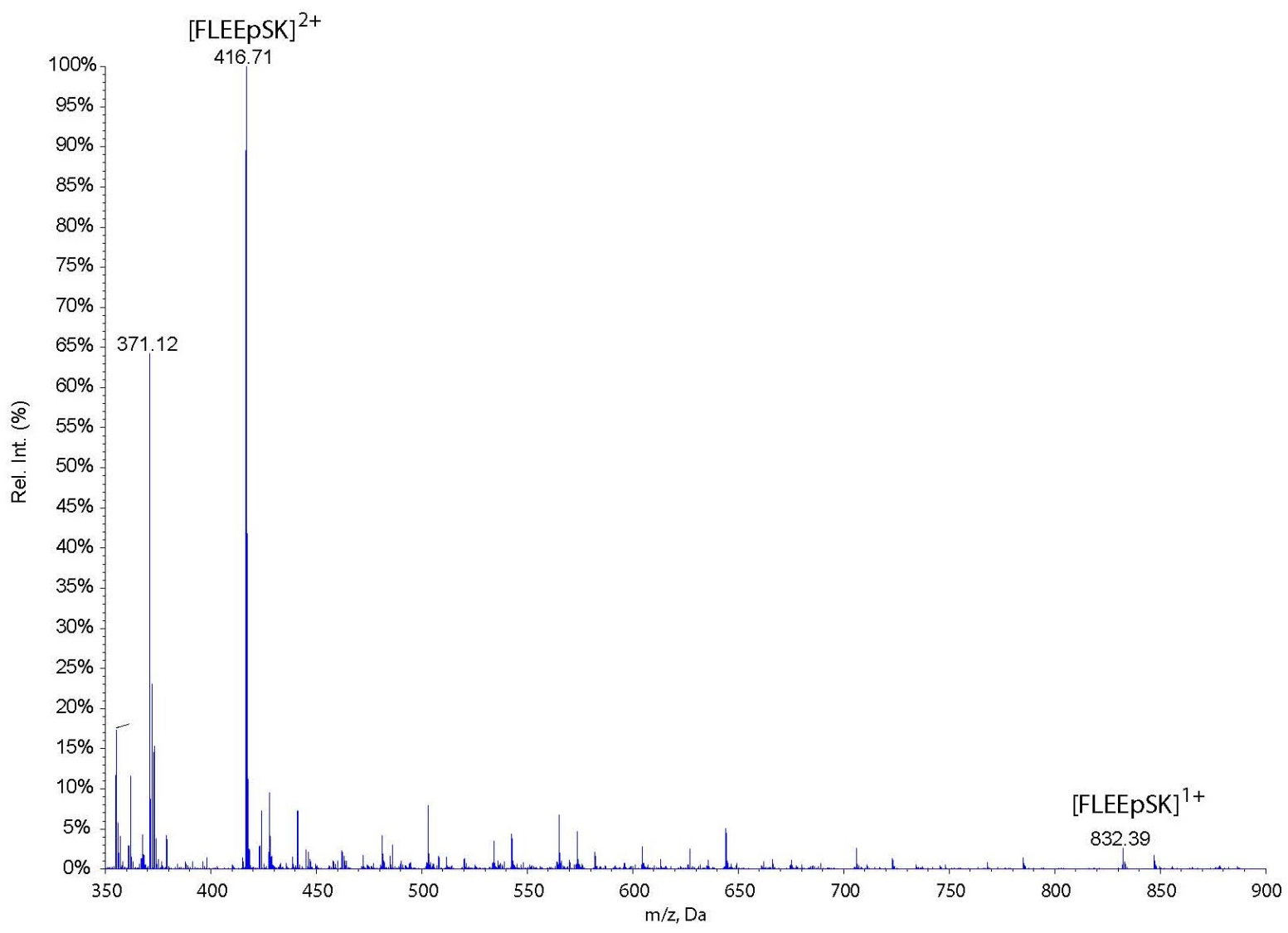

Figure 5.6: MS results of FLEEpSK prior to TrEnDi modification. 


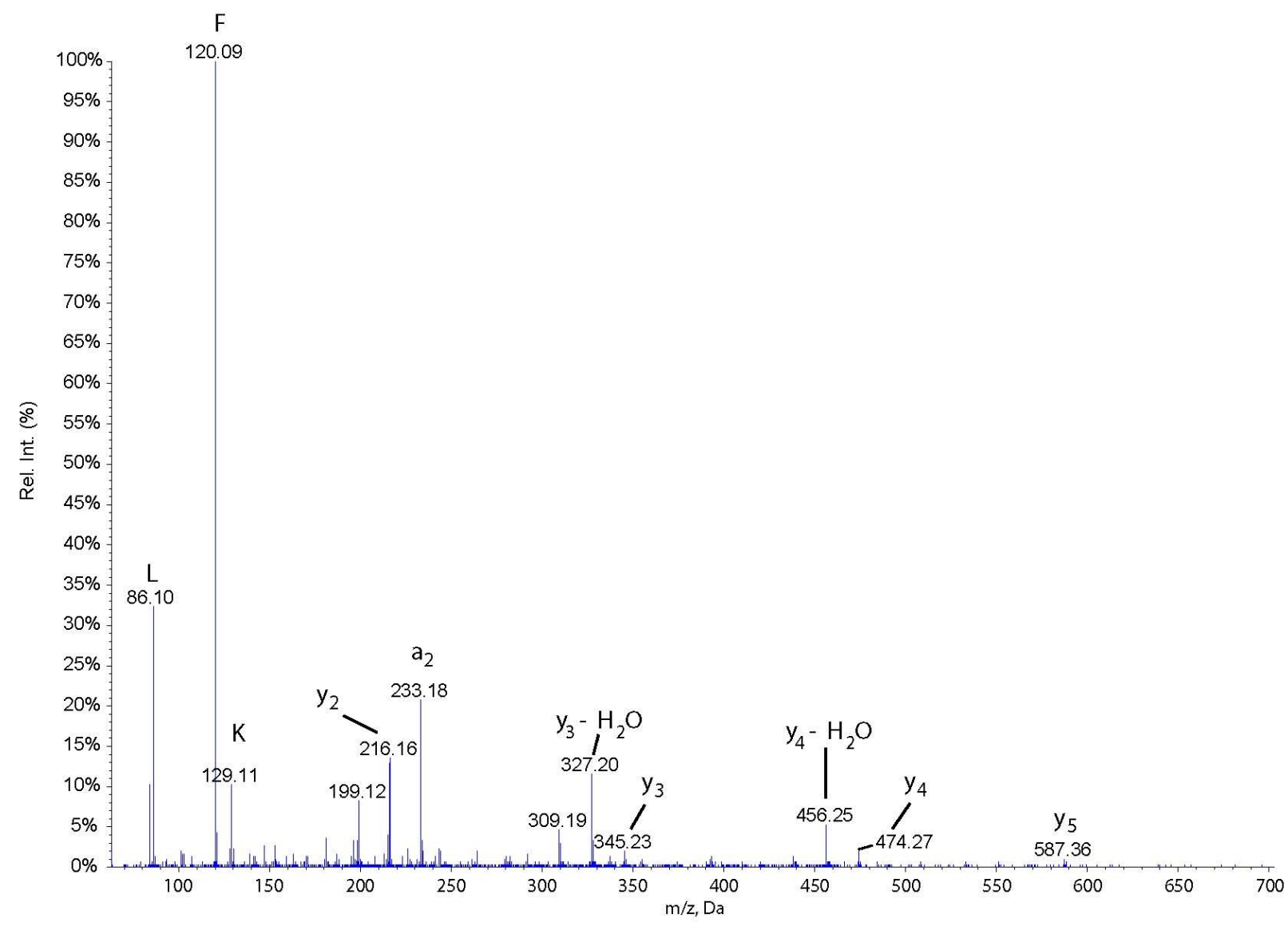

Figure 5.7: $\mathrm{MS}^{2}$ results of unmodified FLEEPSK. All y ions represent the mass of the ion with a neutral loss of $\mathrm{H}_{3} \mathrm{PO}_{4}$ corresponding to a loss of $98 \mathrm{Da}$.

Upon derivatization with diazomethane and elution with sodium hydroxide the final mass-to-charge ratio of the most dominant peak was found to be 409.75 (Figure 5.8). This was an unusual observation upon initial inspection, however, $\mathrm{MS}^{2}$ analysis of 409.75 indicated that FLEEPSK was hexamethylated, that base hydrolysis of the methyl esters was complete and that dephosphorylation of serine occurred somewhere between methylation with diazomethane and elution with sodium hydroxide (Figure 5.9). 


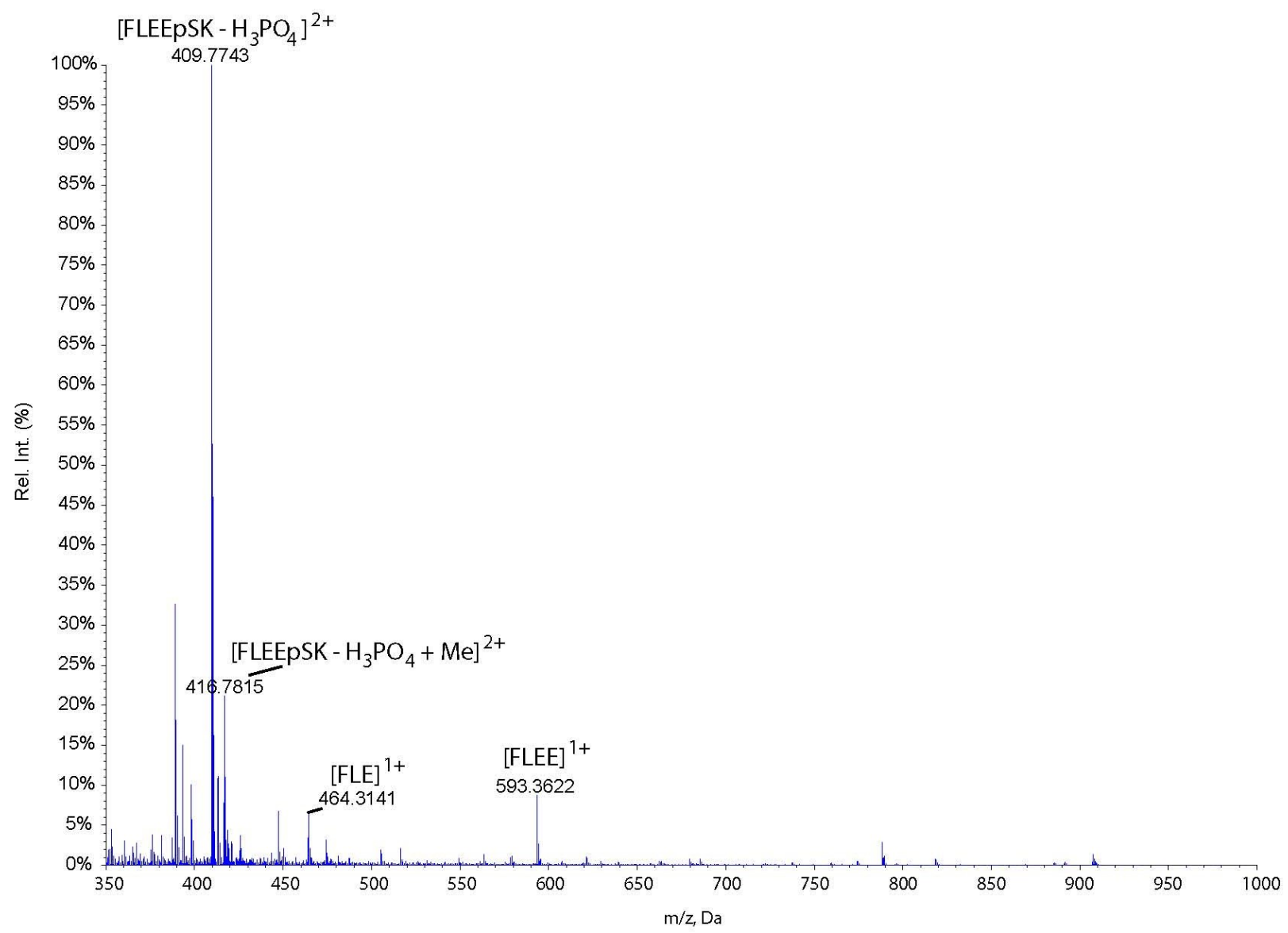

Figure 5.8: MS results of TrEnDi modified FLEEpSK. 


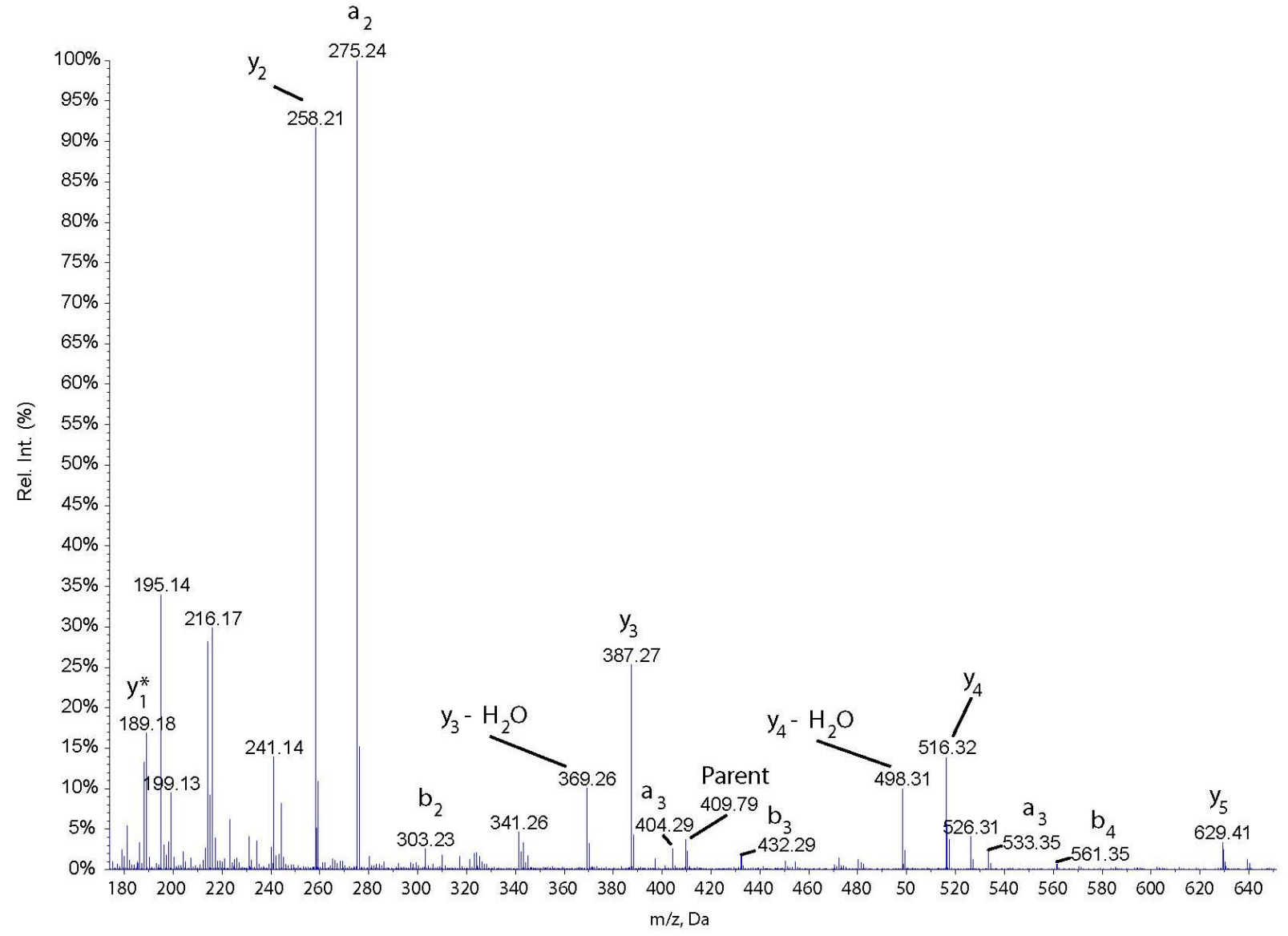

Figure 5.9: $\mathrm{MS}^{2}$ results of modified FLEEpSK at $\mathrm{m} / \mathrm{z}$ 409.7. All $\mathrm{y}$ ions have been dephosphorylated (except $\left.\mathbf{y}_{1}\right)$.

Elution with ammonium bicarbonate studies indicated that a modified phosphate group in FLEEPSK was present with several methylations and that dephosphorylation did not occur during the treatment of diazomethane (data not shown). An additional study showed that elution of unmodified FLEEpSK with $\mathrm{NaOH}$ did not cause dephosphorylation (data not shown). Therefore, the dephosphorylation of TrEnDi modified FLEEpSK was caused by $\mathrm{NaOH}$, though dephosphorylation only occurred after the phosphate group was methylated. The derivatization chemistry and structure of modified FLEEpSK post $\mathrm{NaOH}$ elution is illustrated in Figure 5.10. 


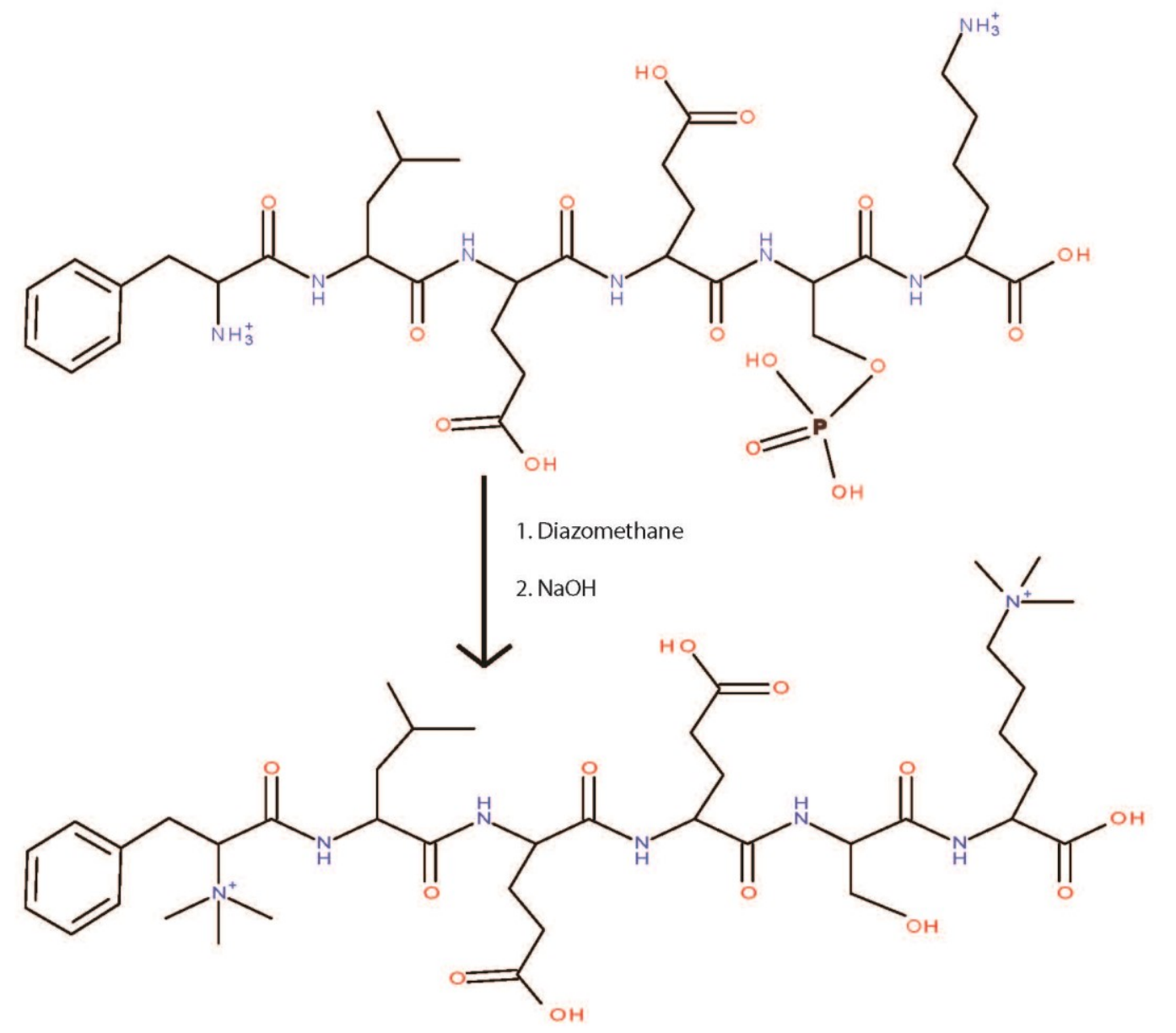

Figure 5.10: Fully modified FLEEpSK with the dephosphorylated serine

Figure 5.8 also reveals a doubly charged species at $\mathrm{m} / \mathrm{z} 416.78$ that would initially cause one to suspect that some FLEEpSK had remained unmodified. However, the $\mathrm{m} / \mathrm{z}$ of the unmodified FLEEPSK is 416.71 whereas the new $\mathrm{m} / \mathrm{z}$ had a shift of +0.07 Da from the unmodified FLEEPSK. $\mathrm{MS}^{2}$ analysis (Figure 5.11) confirmed that the peptide was a fully modified, dephosphorylated FLEEpSK with an additional methyl group. The $\mathrm{MS}^{2}$ analysis was unable to clearly indicate the location of the additional methylation site; therefore it is likely that the additional methylation is occurring on various positions along the back bone of the peptide. 


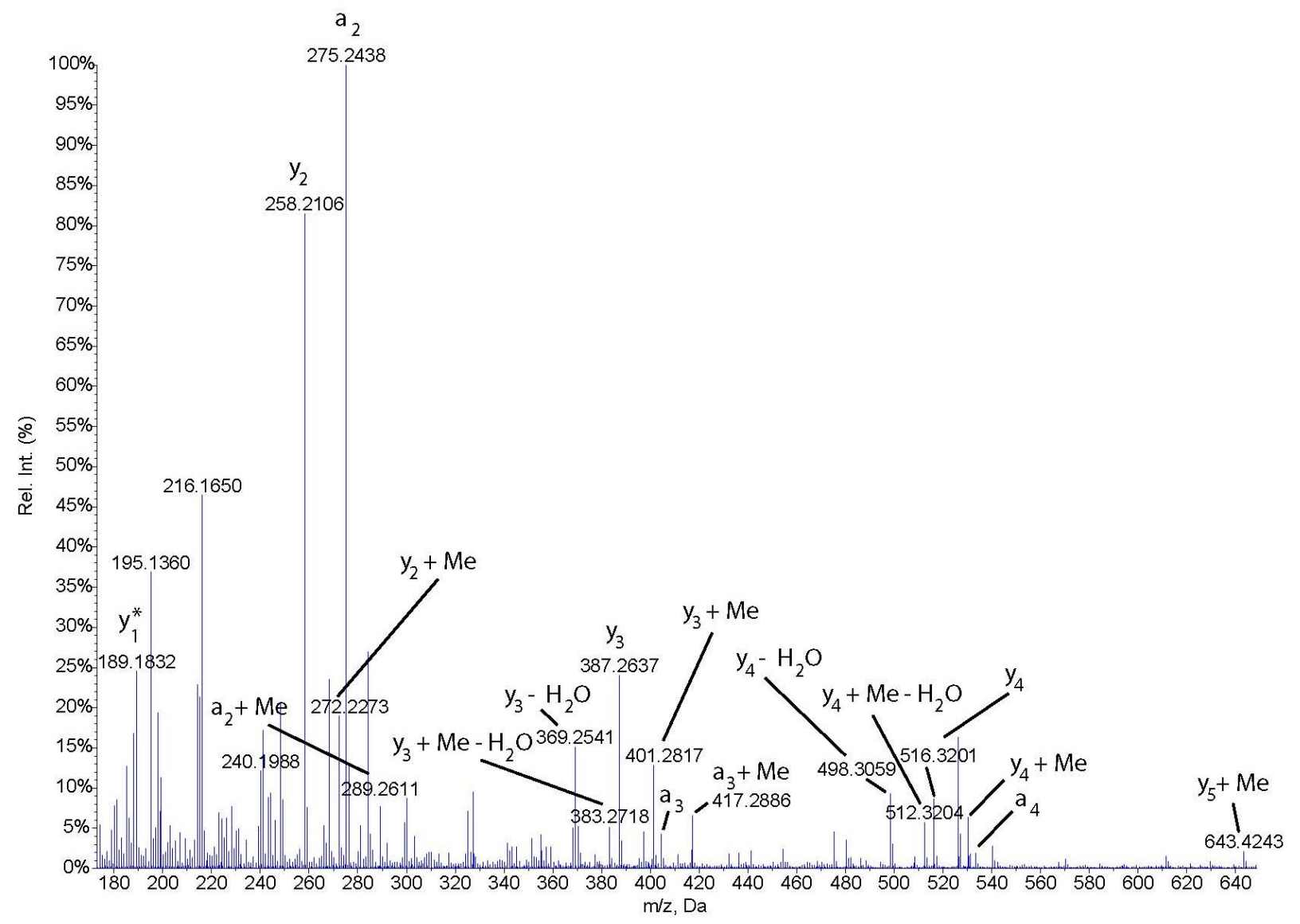

Figure 5.11: $\mathrm{MS}^{2}$ analysis of TrEnDi-modified FLEEpSK with an additional $\mathrm{ME}(\mathrm{m} / \mathrm{z} 416.78)$.

Further TrEnDi studies on phosphorylated peptides were performed on phosphorylated tyrosine in the peptide FLEEPYK. Figure 5.12 and Figure 5.13 show the MS and the $\mathrm{MS}^{2}$ analysis of unmodified FLEEPYK. Previous studies have shown that tyrosine residues remain methylated even when subjected to base hydrolysis using $\mathrm{NaOH}$ (section 3.4). It was hypothesized that a phosphorylated tyrosine subjected to TrEnDi and base hydrolysis would dephosphorylate. This would provide a unique method for determining the location of phosphorylated tyrosine residues on a peptide. In theory, a tyrosine residue that was not phosphorylated would get methylated when subjected to diazomethane treatment, whereas 
tyrosine residues that were phosphorylated would dephosphorylate and remain unmodified when subjected to diazomethane treatment and base hydrolysis with $\mathrm{NaOH} . \mathrm{MS}^{2}$ analysis would provide details as to whether the tyrosine residue was methylated or not. Methylation would indicate that there was no phosphate group present prior to TrEnDi treatment, whereas an unmodified tyrosine residue would indicate that there was a phosphate group present prior to TrEnDi treatment and base hydrolysis.

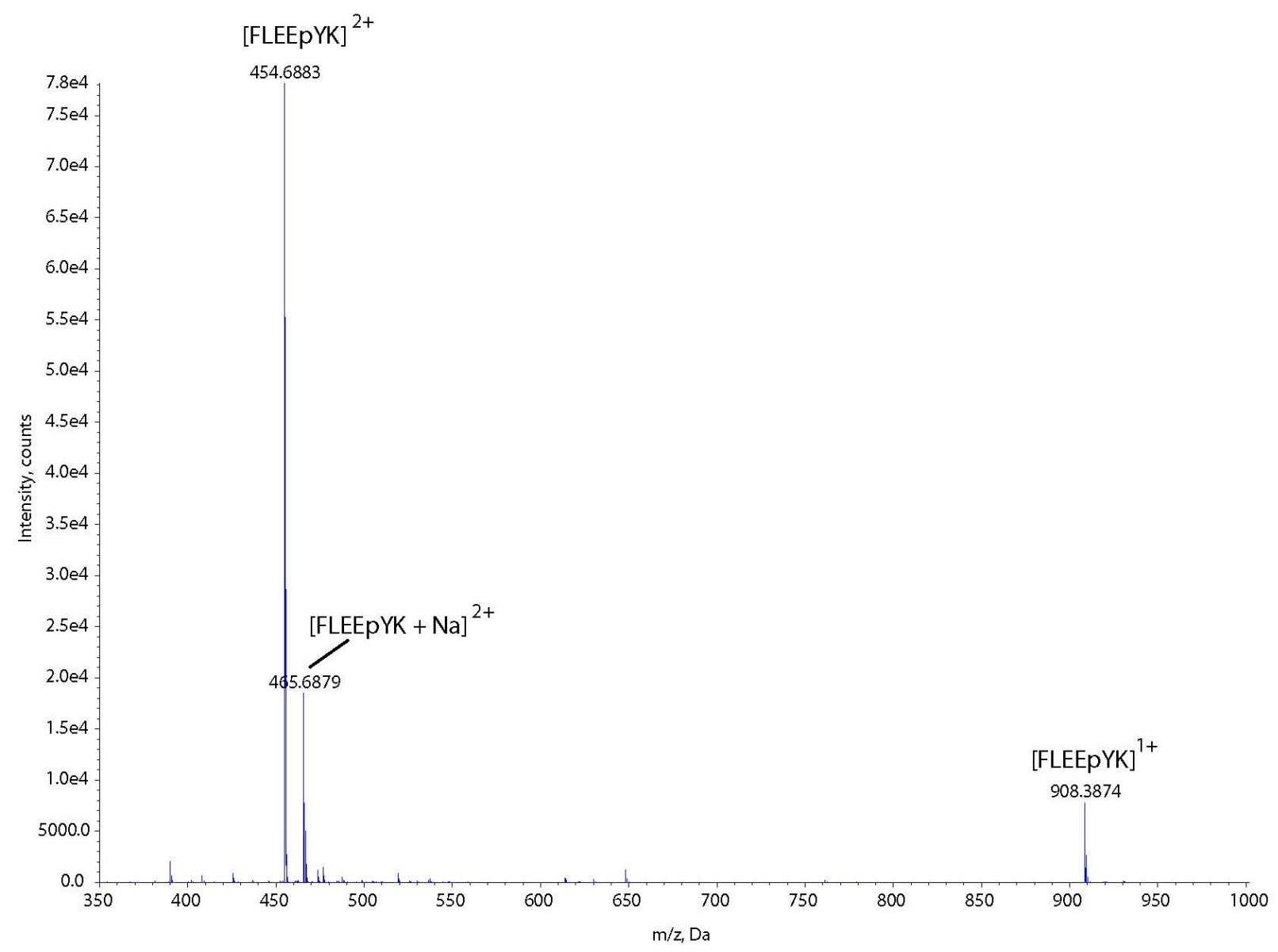

Figure 5.12: MS analysis of unmodified FLEEpYK 


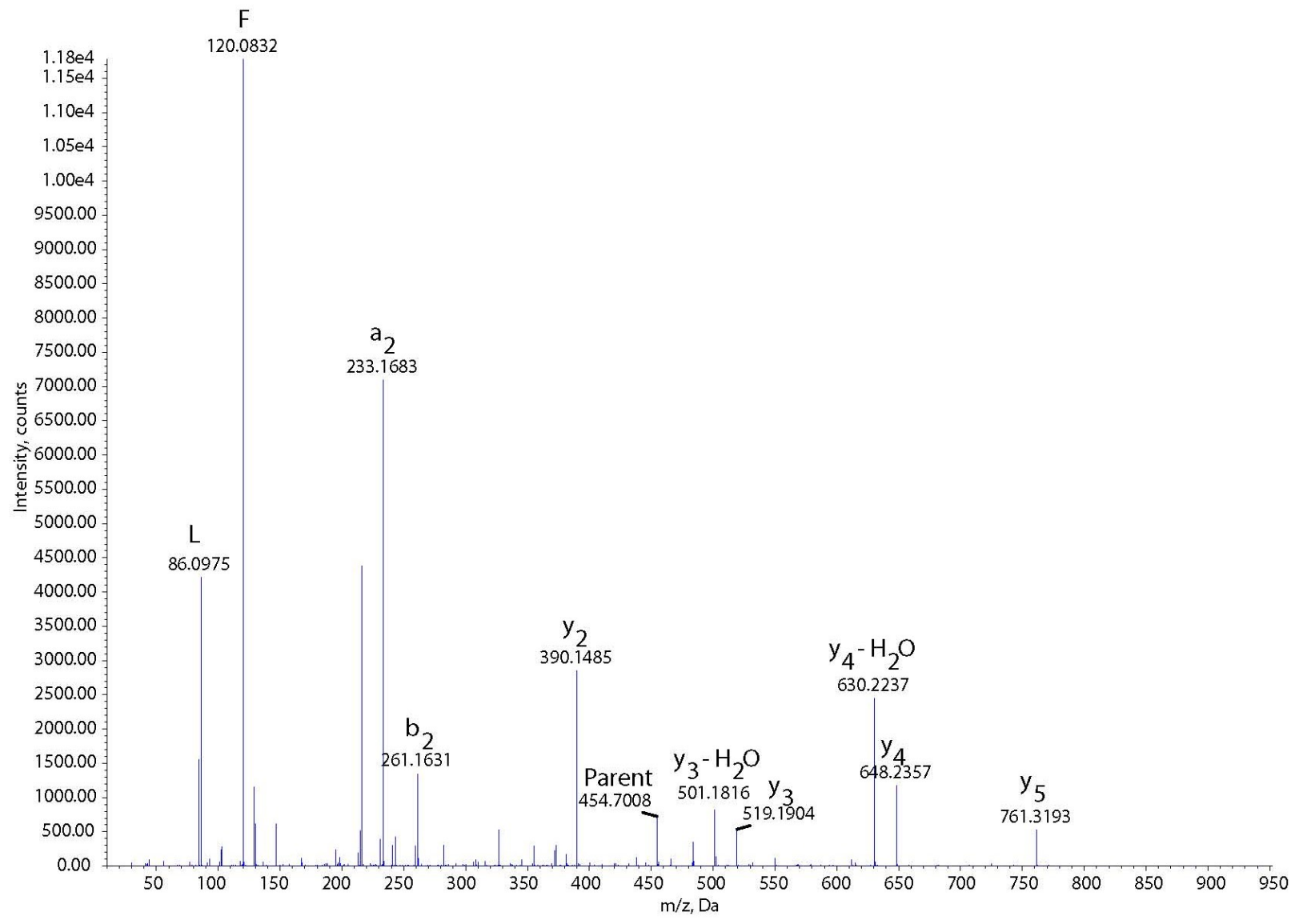

Figure 5.13: $\mathrm{MS}^{2}$ analysis of unmodified FLEEpYK at $\mathrm{m} / \mathrm{z}$ 454.7. All $\mathrm{y}$ ions represent the mass of the ion with a neutral loss of $\mathrm{H}_{3} \mathrm{PO}_{4}$ corresponding to a loss of $98 \mathrm{Da}$

In testing our hypothesis, results indicated that our initial hypothesis was nearly correct. The $\mathrm{m} / \mathrm{z}$ of 456.77 in Figure 5.14 was the exact $\mathrm{m} / \mathrm{z}$ of the predicted dephosphorylated hexamethylated FLEEPYK with an unmodified tyrosine residue. This was confirmed by $\mathrm{MS}^{2}$ analysis (see Figure 5.15); however, Figure 5.14 also reveals two related ions at $\mathrm{m} / \mathrm{z}$ of 463.77 and 503.76. As with FLEEpSK, the $\mathrm{m} / \mathrm{z}$ of 463.77 was found to be the dephosphorylated FLEEpYK with an additional methyl group. 


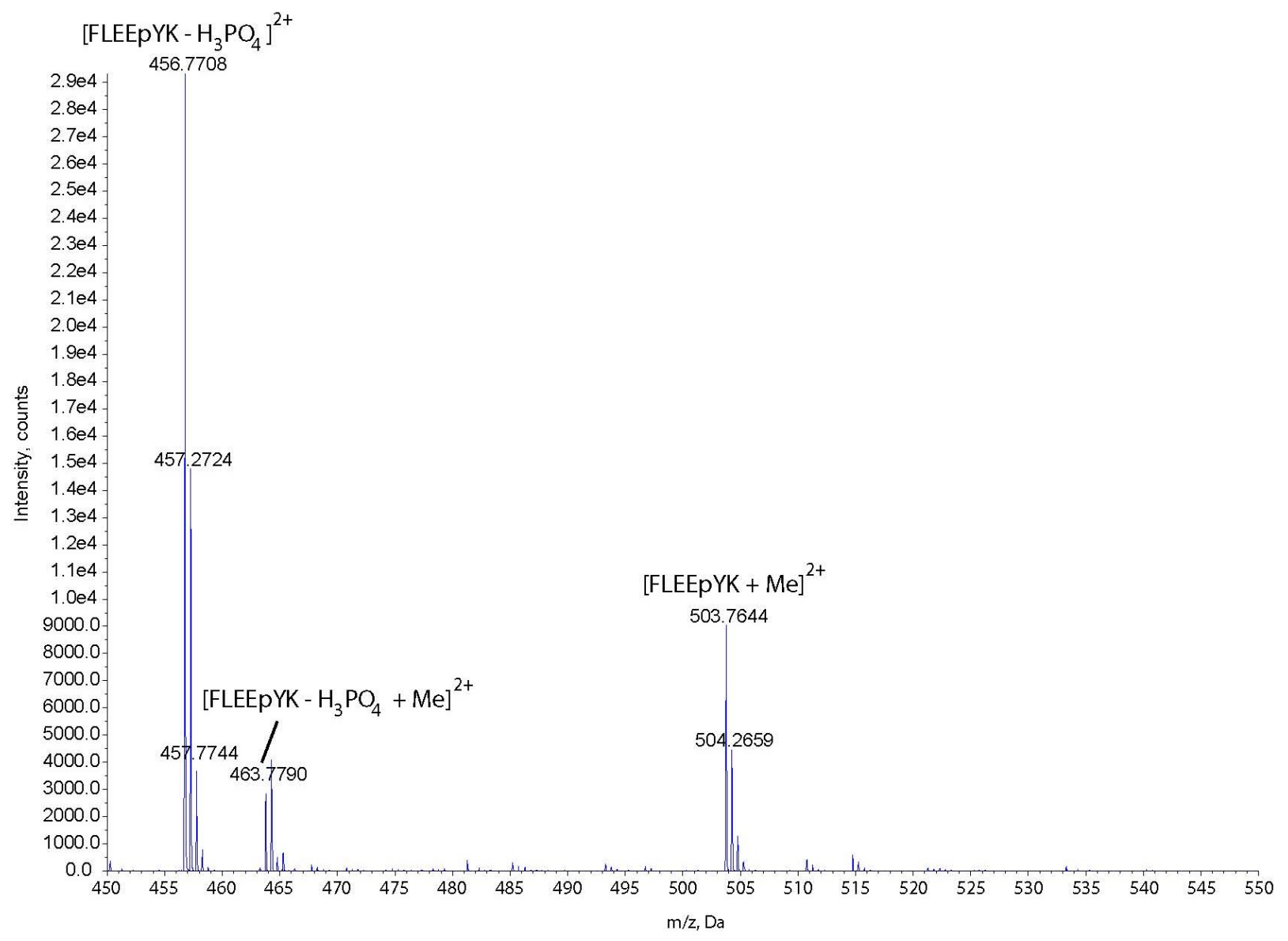

Figure 5.14: MS analysis of on-column TrEnDi modified FLEEpYK

$\mathrm{MS}^{2}$ analysis is unable to determine one exact location of the additional methylation site as there is evidence of an additional methylation on both the a and $y$ ion series; however, based on intensity, Figure 5.16 would suggest that the majority of the additional methylation is occurring on the backbone between $\mathrm{y}_{1}$ and $\mathrm{y}_{2}$. Ultimately, the intensity of $\mathrm{m} / \mathrm{z} 463.77$ in Figure 5.14 is the sum of all the modified FLEEPYK with one extra methylation. 


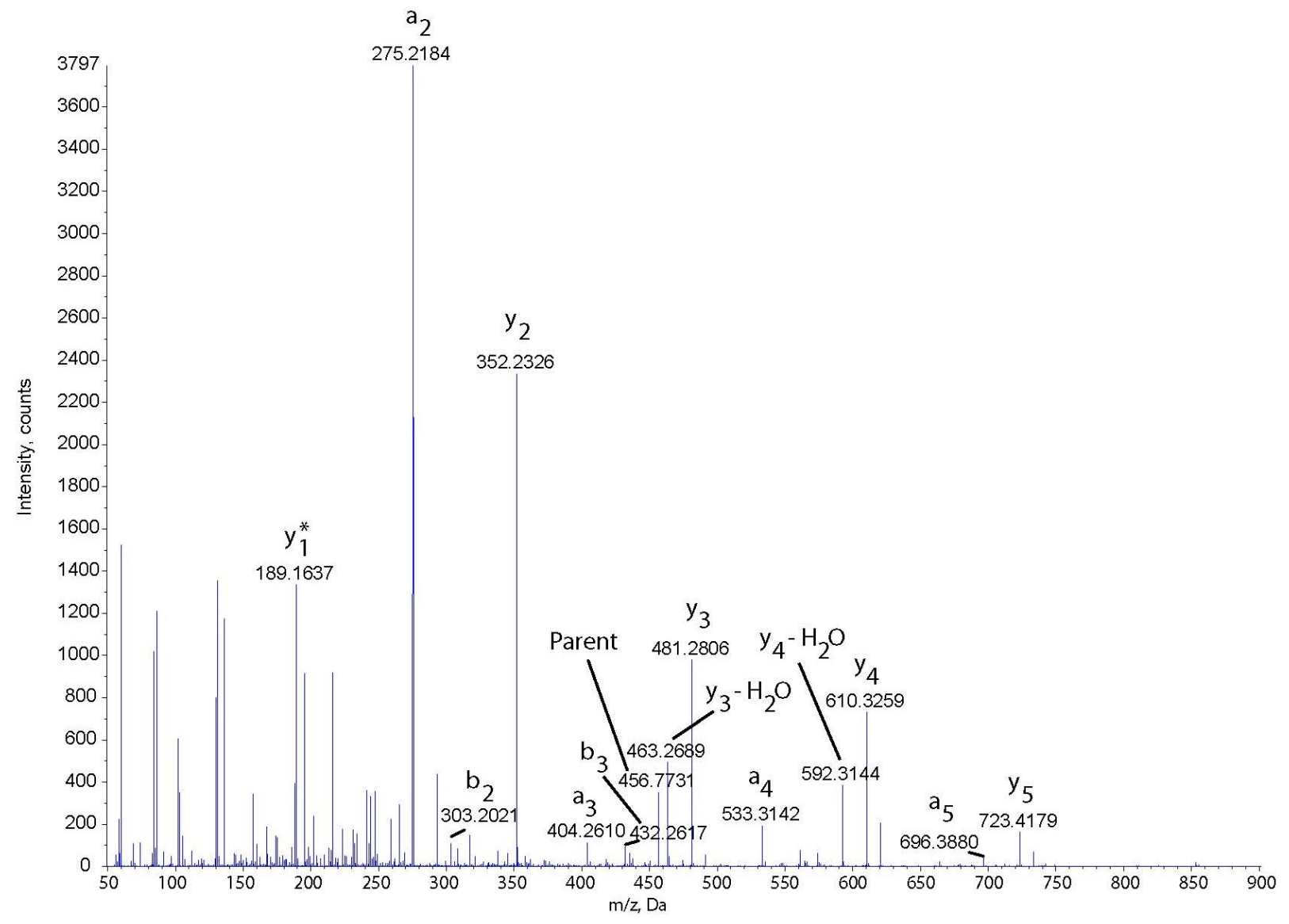

Figure 5.15: $\mathrm{MS}^{2}$ analysis of $\mathrm{m} / \mathrm{z}$ 454.7. All $\mathrm{y}$ ions are fragment masses of the dephosphorylated parent ion except $\mathrm{y}_{1}$. 


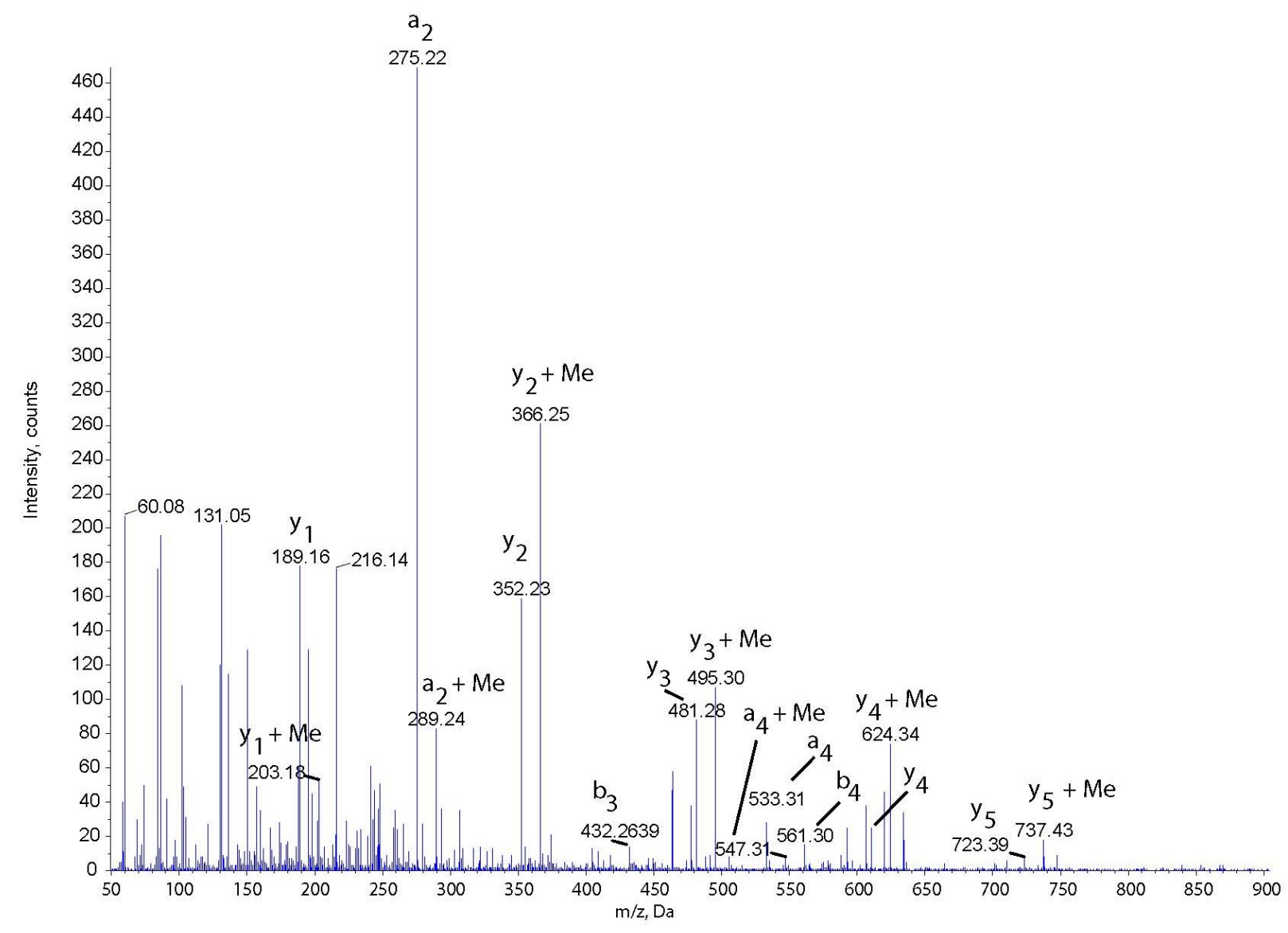

Figure 5.16: $\mathrm{MS}^{2}$ analysis of $\mathrm{m} / \mathrm{z}$ 463.7. All $\mathrm{y}$ ions are fragment masses of the dephosphorylated parent ion except $\mathrm{y}_{1}$.

$\mathrm{MS}^{2}$ analysis of $\mathrm{m} / \mathrm{z} 503.76$ not only indicated that some of the phosphate group remained present on the peptide, but that the phosphate group was singly methylated (Figure 5.17). Therefore, at some point during base hydrolysis, though occurring at a less favourable rate, one of the newly formed phosphate methyl esters was hydrolysing prior to dephosphorylation. This would suggest that hydrolysis of just one of the newly formed phosphate methyl esters would stabilize the phosphate group preventing dephosphorylation. Therefore, we were not successful at driving the reaction to a single dephosphorylated product. 
In an attempt to drive the fully modified FLEEPYK to one product, a neutral salt elution solution that would not perform base hydrolysis was explored.

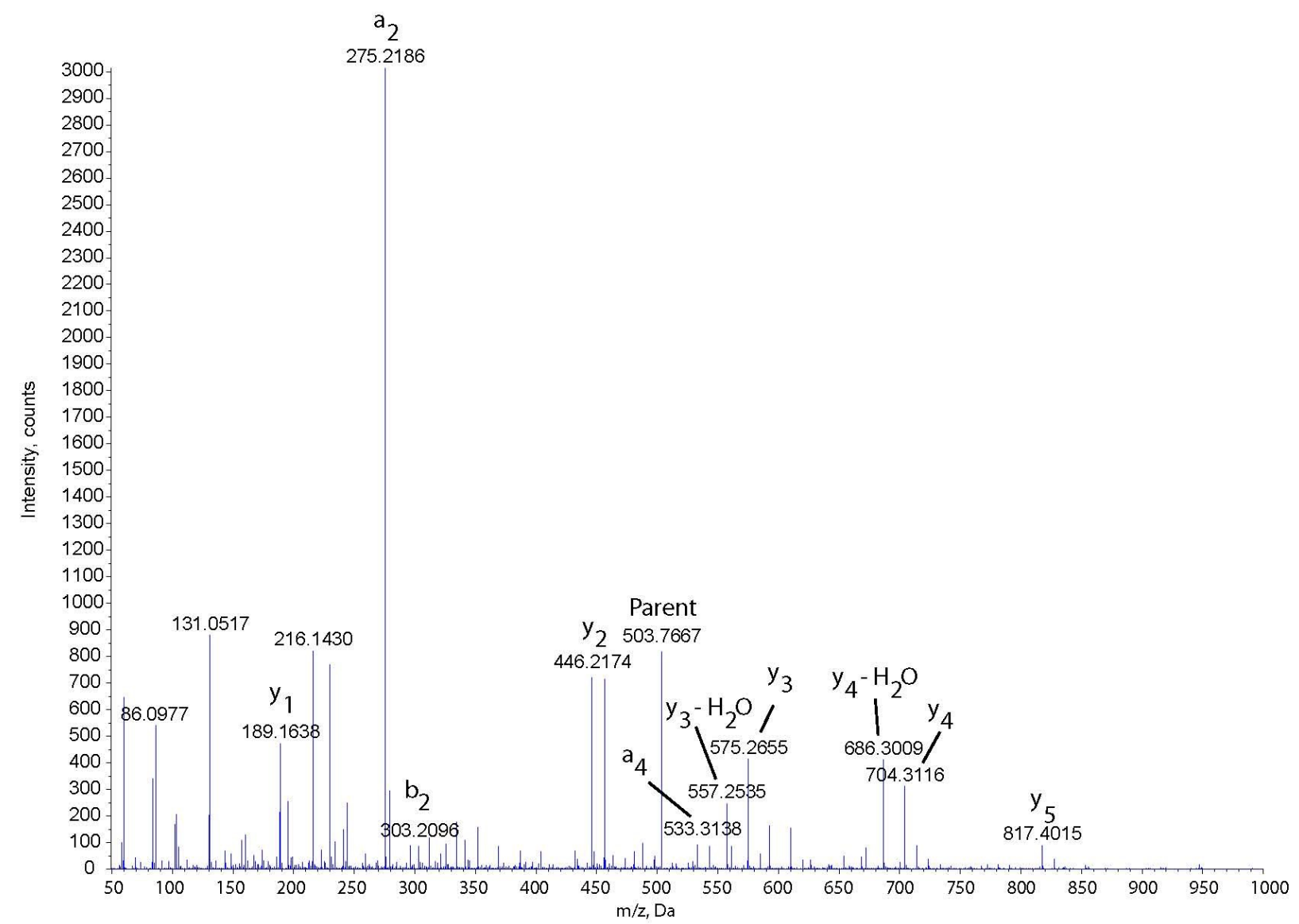

Figure 5.17: $\mathrm{MS}^{2}$ analysis of $\mathrm{m} / \mathrm{z} 503.7$ with the phosphate group still intact.

As a single preliminary test on FLEEpYK, a packet of iodized salt obtained from Carleton University's University Centre Cafeteria was dissolved in $8 \mathrm{~mL}$ of deionized Milli Q water. 100 $\mu \mathrm{L}$ of this salty solution was used to elute FLEEpYK post on-column TrEnDi modification. After desalting (section 2.4), MS analysis showed a fascinating result (Figure $5.18 \mathrm{~A}$ and $\mathrm{B}$ ) indicating a fully modified FLEEPYK product with 11 methyl groups including three methyl esters and two on the phosphate group as indicated in Figure 5.19. Figure 5.18 B shows an unusual isotopic 
distribution for the peak at $\mathrm{m} / \mathrm{z} 531.78$. This was due to the oversaturation of the detector by a highly concentrated sample. This result reveals that under relatively neutral conditions, the modified phosphate group will remain intact. Recent improvements on elution without base hydrolysis are now feasible (section 5.3). This new elution technique could prove to be useful for further phosphoproteomic studies as the phosphate groups would remain fully modified, hence neutralizing their negative charge while providing the benefits of TrEnDi to the rest of the peptide. This test remains to be studied.

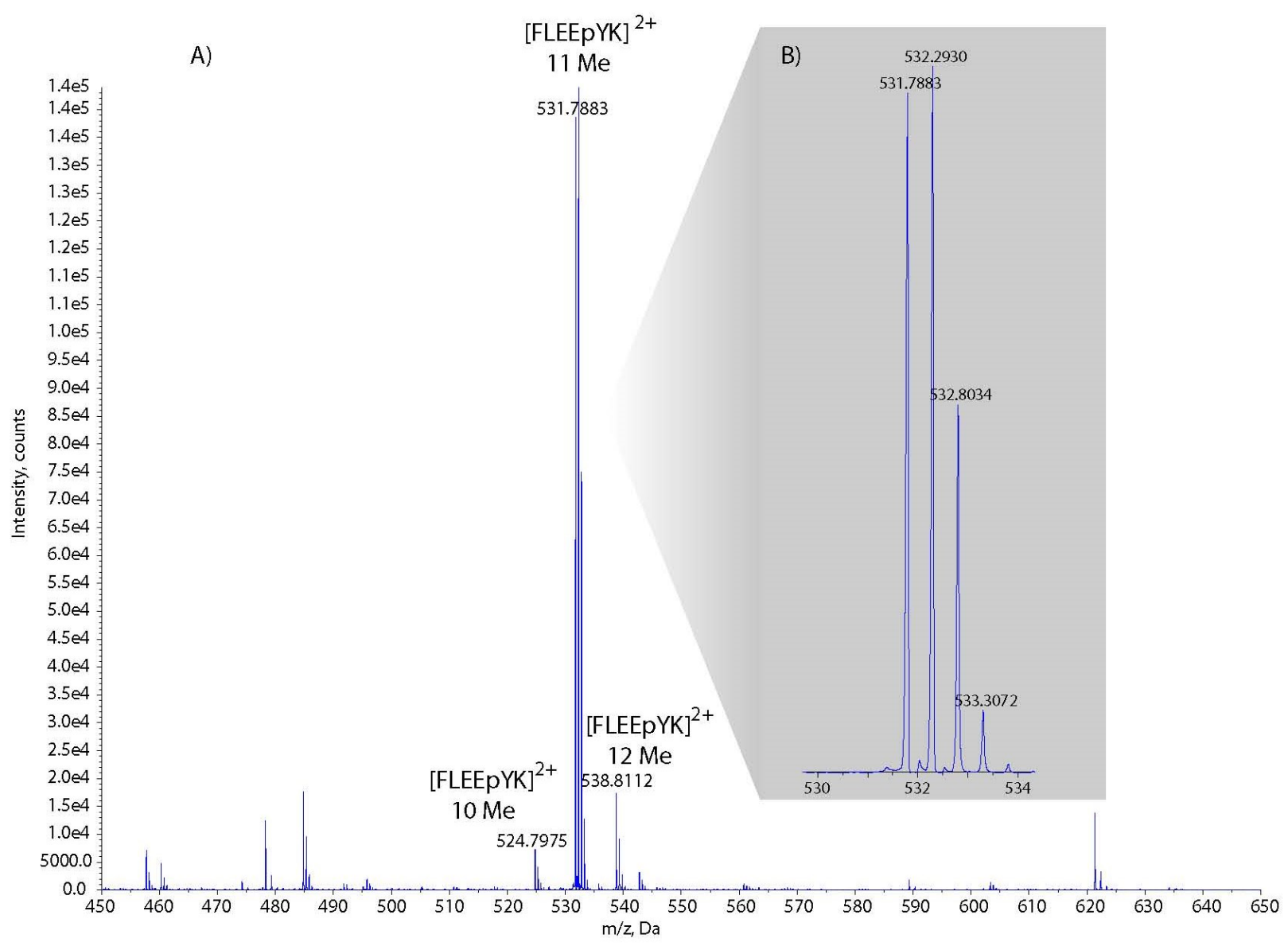

Figure 5.18: A) MS analysis of TrEnDi modified FLEEpYK eluted with iodized salt. B) Zoom in on the saturation of $\mathrm{m} / \mathrm{z} 531.78$ 


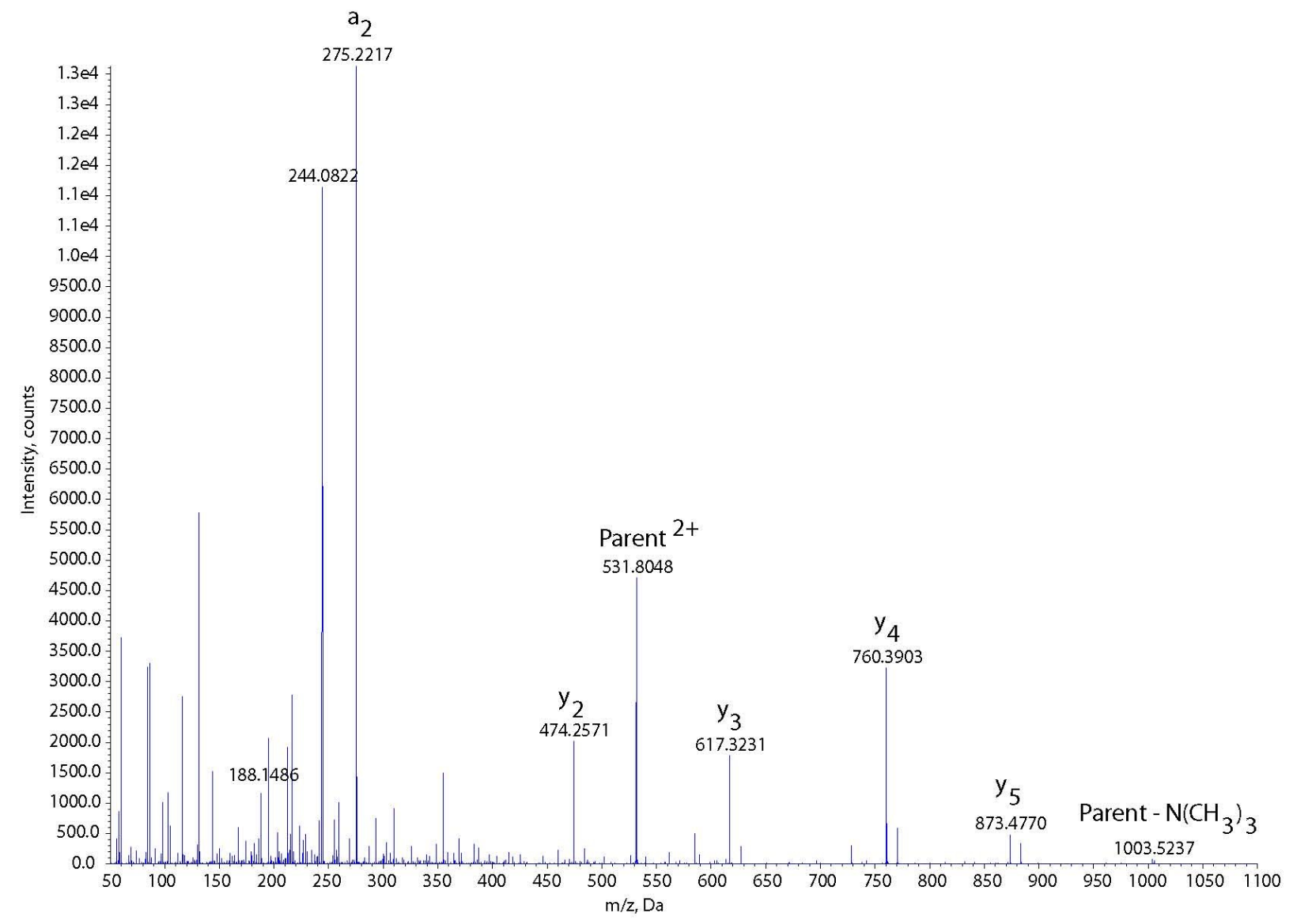

Figure 5.19: $\mathrm{MS}^{2}$ analysis of $\mathrm{m} / \mathrm{z}$ FLEEpYK with methyl esters and dimethyl phosphate still intact.

\subsection{1 $\mathrm{Na}^{18} \mathrm{OH}$ elution studies}

As an additional feature to $\mathrm{TrEnDi},{ }^{18} \mathrm{O}$ labelling studies were performed to determine the applicability of labelling peptides with ${ }^{18} \mathrm{O}$ during base hydrolysis. Work was performed on FLEEPSK peptide on-column following the general procedures found in section 2.3. Elution of peptides was performed using $60 \mu \mathrm{L}$ of $\sim 1 \mathrm{M} \mathrm{Na}{ }^{18} \mathrm{OH}$. Production of $\mathrm{Na}{ }^{18} \mathrm{OH}$ was described in section 2.7. Elution studies with $\mathrm{Na}^{18} \mathrm{OH}$ have shown to provide TrEnDi with the means of ${ }^{18} \mathrm{O}$ labelling during the hydrolysis of the newly formed methyl esters. As previously described, 
elution with $\mathrm{Na}^{18} \mathrm{OH}$ was able to dephosphorylate serine and label all acidic residues. Base hydrolysis with $\mathrm{Na}^{18} \mathrm{OH}$ provides us with the unique advantage of modifying peptides in such a way that there will be a minimum of 2 Da mass shift for every peptide subjected to TrEnDi and TrEnDi with $\mathrm{Na}^{18} \mathrm{OH}$ elution. An example is provided with FLEEpSK, where a $\mathrm{m} / \mathrm{z}$ shift of $3 \mathrm{Da}$, equivalent to six mass units, is observed (see Figure 5.20 and Figure 5.8). This now offers another means for quantitative analysis, such that two of the same analytes from different samples can be analyzed together simultaneously and the relative difference in their intensities can be measured. $\mathrm{MS}^{2}$ comparison between FLEEpSK eluted with $\mathrm{Na}^{16} \mathrm{OH}$ and FLEEpSK eluted with $\mathrm{Na}^{18} \mathrm{OH}$ is shown in Figure 5.21. The derivatization chemistry and structure of modified FLEEpSK followed by $\mathrm{Na}^{18} \mathrm{OH}$ elution is illustrated in Figure 5.22 .

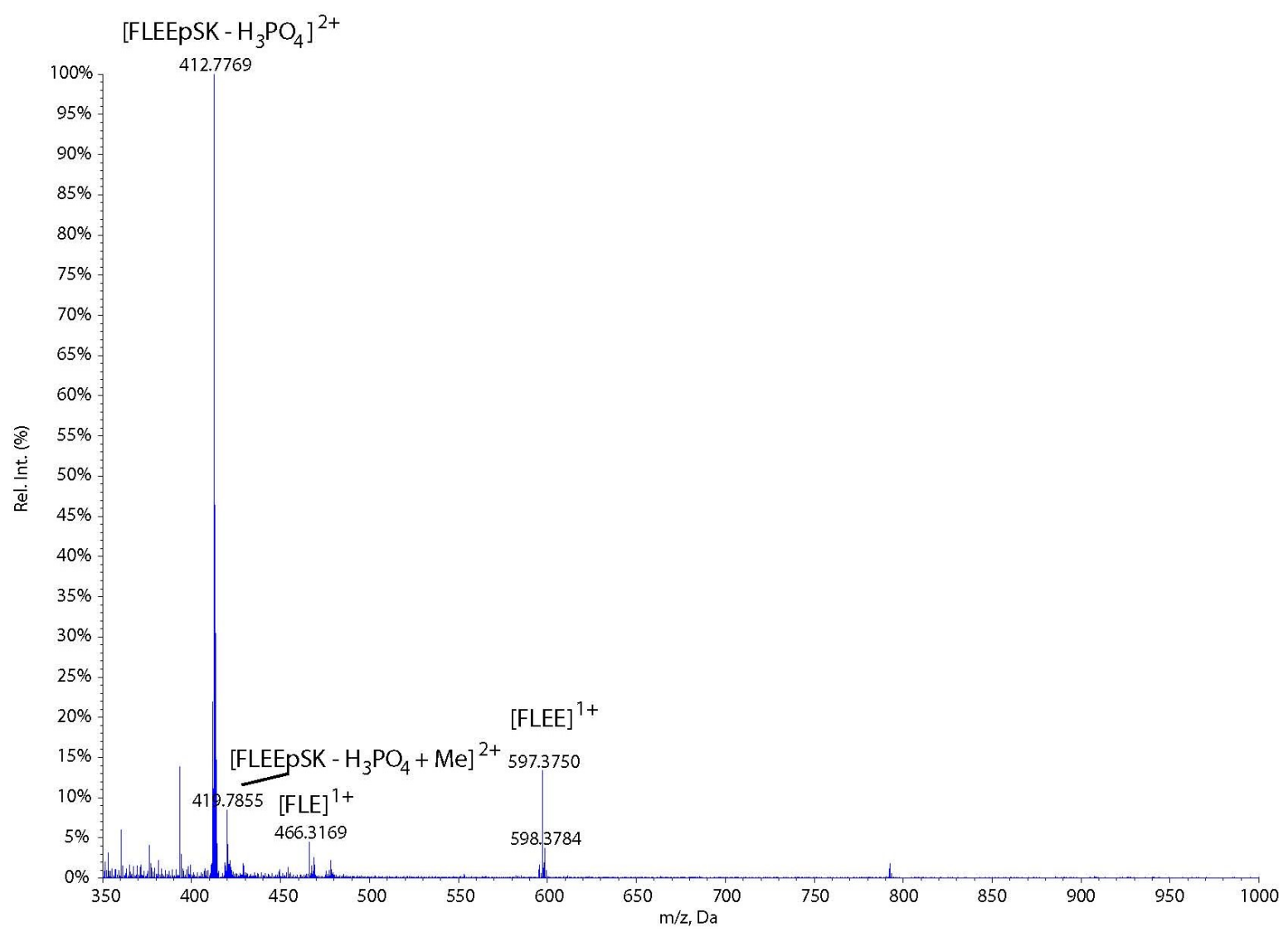

Figure 5.20: MS of TrEnDi modified FLEEpSK and elution with $\mathrm{Na}^{18} \mathrm{OH}$. 

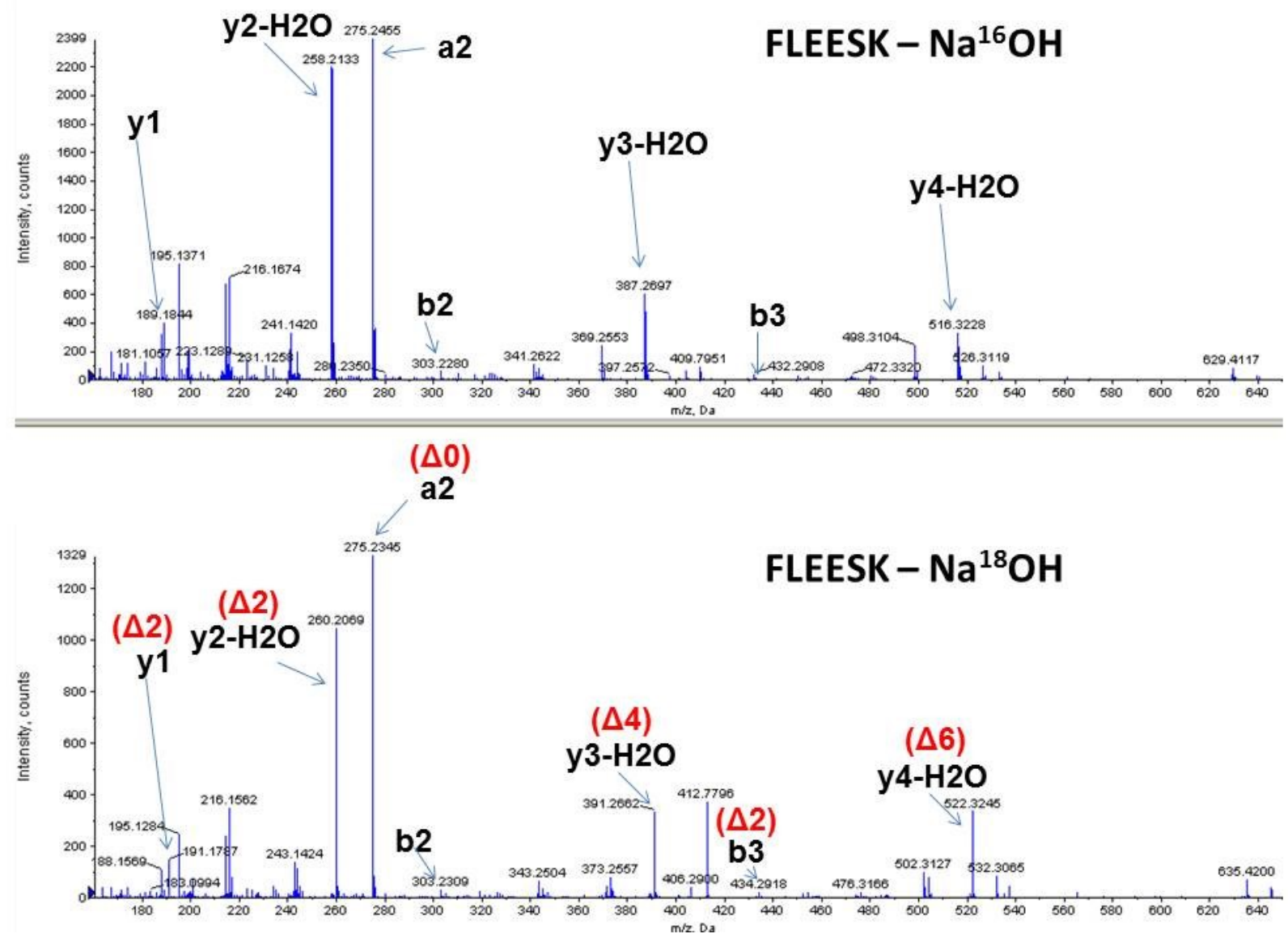

Figure 5.21: $\mathrm{MS}^{2}$ comparison between TrEnDi modified FLEEpSK and elution with $\mathrm{Na}^{16} \mathrm{OH}$ and $\mathrm{Na}^{18} \mathrm{OH}$ 


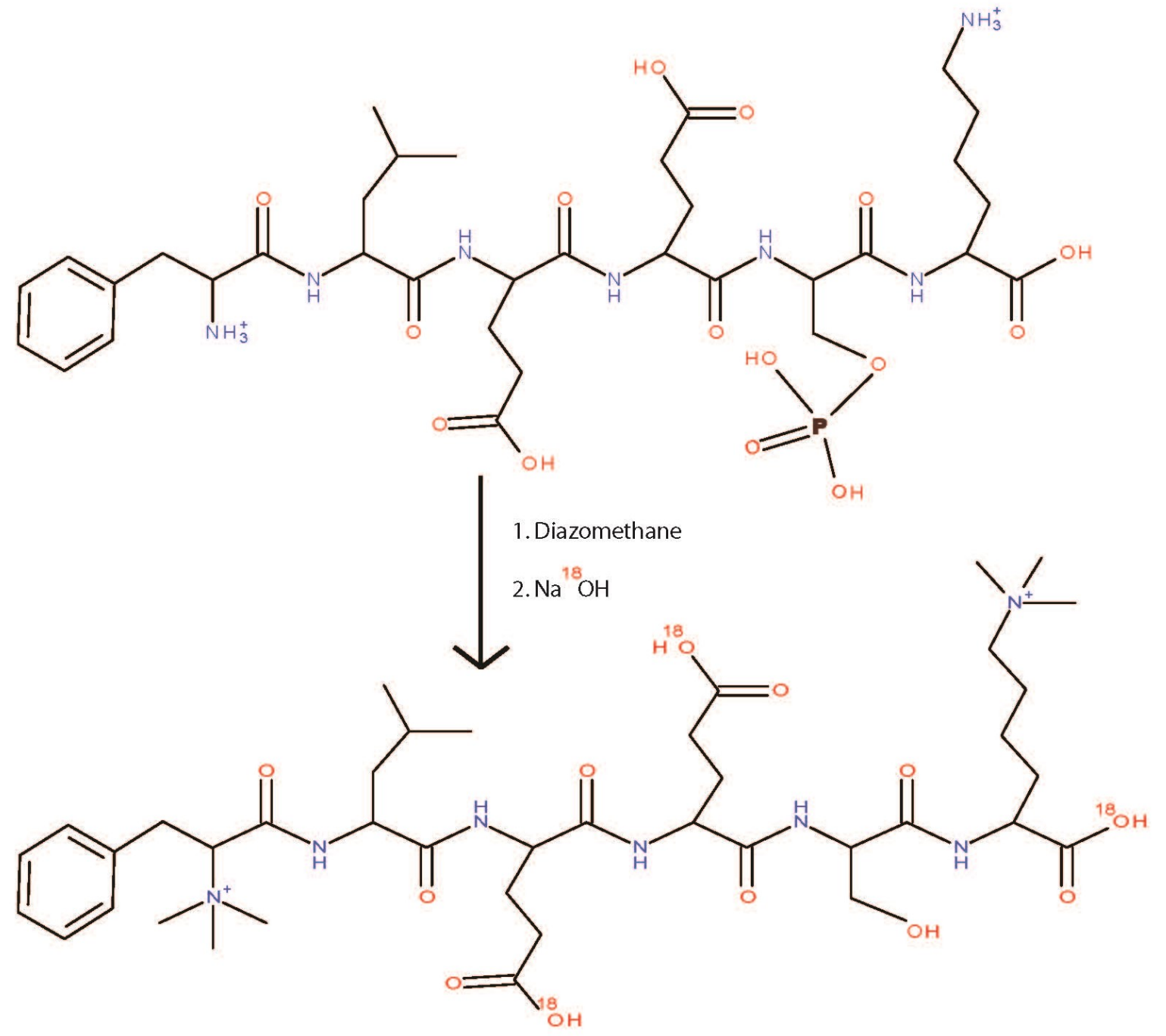

Figure 5.22: Fully modified FLEEpSK via treatment with diazomethane and $\mathrm{Na}^{18} \mathrm{OH}$

\subsection{On-column derivatization and elution with triethylammonium acetate}

Derivatization and elution with triethylammonium acetate was performed following the general procedure for on-column derivatization and desalting found in section 2.3 and 2.4. Approximately $100 \mu \mathrm{L}$ of triethylammonium acetate was used during the elution step. The 
purpose of this experiment was to determine whether or not a solution with neutral $\mathrm{pH}$ could be used to elute peptides from the SCX resin. The hypothesis was that triethylammonium acetate, being a volatile salt and an improvement over iodized salt, would provide sufficient enough competition for binding sites on the SCX resin to elute our analyte, while preventing acid or base hydrolysis of the newly formed methyl esters.

Elution with triethylammonium acetate was attempted on the combination of FLEEVR, FLEEVK, FLEENK and FLEEYK peptides. Figure 5.23 reveals the mass spectrum of diazomethanemodified FLEEVR, FLEEVK, FLEENK and FLEEYK following elution with $\mathrm{Et}_{3} \mathrm{~N}^{+} \mathrm{H}^{-} \mathrm{OAc}$. A total of six methylations on FLEEVR, nine methylations on FLEEVK and FLEENK and ten methylations on FLEEYK were observed, confirmed by the dominant molecular ions $\left[\right.$ FLEEVR] ${ }^{2+}, \quad[\text { FLEEVK }]^{2+}$, $\left.{ }_{[F L E E N K}\right]^{2+}$ and $\left[\right.$ FLEEYK] ${ }^{2+}$ at $\mathrm{m} / \mathrm{z} 438.7,445.7,453.2$ and 484.7 respectively. Trimethylation of the $\mathrm{N}$-terminus as well as the primary amine of the lysine side chain was confirmed by $\mathrm{MS}^{2}$ analysis (Figure 5.25 to Figure 5.28); the preservation of the methyl esters was also confirmed. 


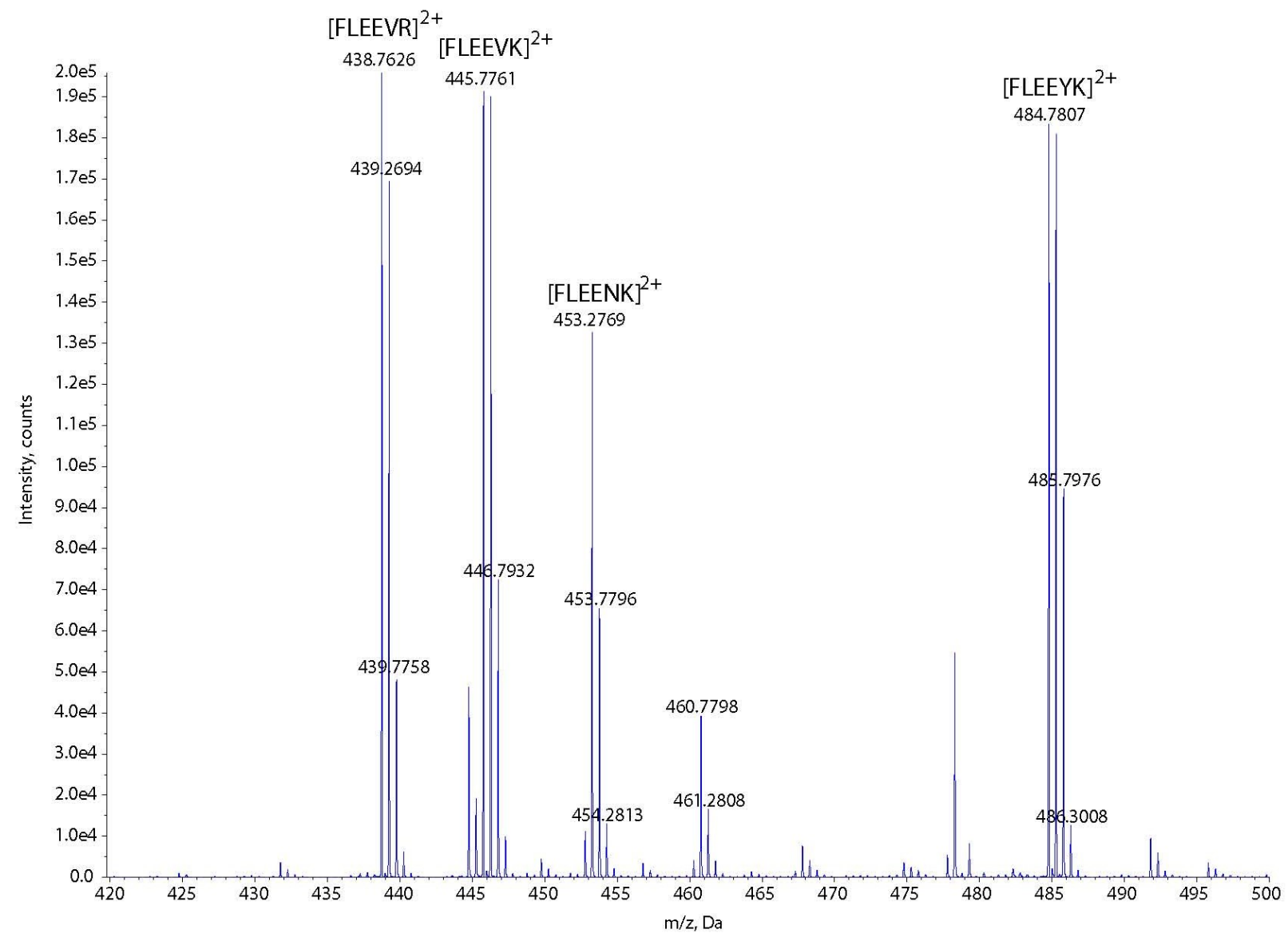

Figure 5.23: MS analysis of fully modified FLEEVR, FLEEVK, FLEENK and FLEEYK post TrEnDi modification and elution with $\mathrm{Et}_{3} \mathrm{~N}^{+} \mathrm{H}^{-} \mathrm{OAc}$

The overall reaction of FLEEVK with diazomethane followed by $\mathrm{Et}_{3} \mathrm{~N}^{+} \mathrm{H}^{-} \mathrm{OAc}$ elution is illustrated in Figure 5.24. The results of the treatment of each peptide with diazomethane on an SCX column followed by $\mathrm{Et}_{3} \mathrm{~N}^{+} \mathrm{H}^{-} \mathrm{OAc}$ elution are listed in Table 5.6. 


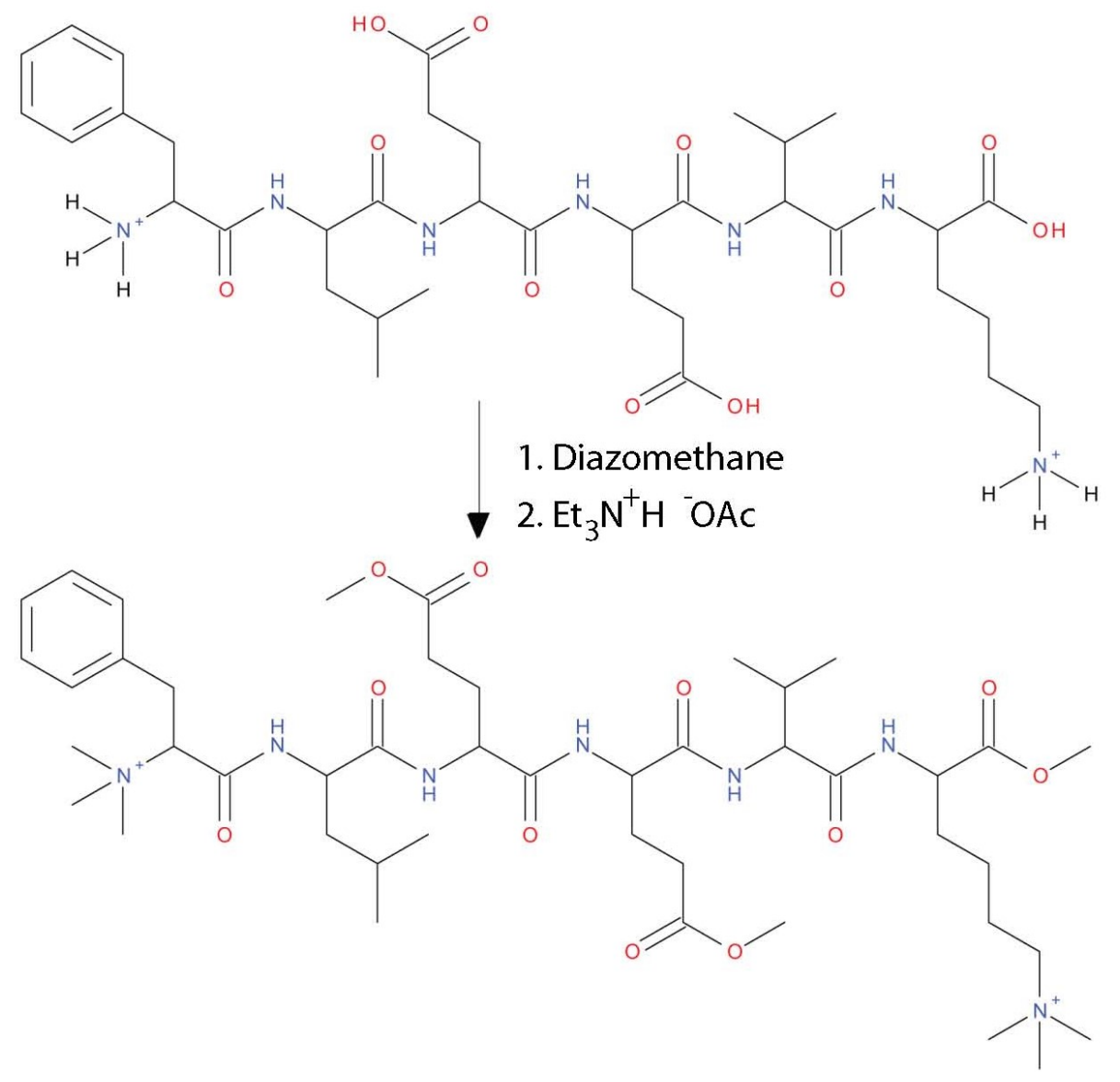

Figure 5.24: FLEEVK subjected to diazomethane treatment and triethylammonium acetate elution yields a fully modified FLEEVK with 9 methylations and 2 fixed positive charges

Elution with triethylammonium acetate now provides us with the unique ability to fully modify peptides, including the methyl esters. This may improve analysis of full proteins. As was previously discussed, multiply charged peptides can become extremely hydrophilic, especially after base hydrolysis of the methyl esters. It is possible that elution with triethylammonium acetate will increase the hydrophobicity with the methyl esters and allow for further detection of peptides following the desalting procedure. Furthermore, triethylammonium acetate is a volatile salt. Therefore, the elution solution post-TrEnDi can be 
evaporated and the modified peptides could be reconstituted in a more desirable spraying solution. These hypotheses have yet to be tested. In addition to these advantages, further ${ }^{13} \mathrm{C}$ vs. ${ }^{12} \mathrm{C}$ diazomethane studies will provide large differences in $\mathrm{m} / \mathrm{z}$ ratio, which will enable one to perform further quantitative analysis. Furthermore, elution with triethylammonium acetate may provide further advancement in phosphoproteomic studies as the fully modified phosphate group would likely remain fully modified and intact. A fully modified phosphate group removes the negative charge, thereby rendering that portion of the peptide as neutral, while fixing positive charges to the $\mathrm{N}$-terminus, histidine residues, and any lysine side chains. This modification of the phosphate group would allow one to confirm the location of this common PTM via $\mathrm{MS}^{2}$ analysis. These studies could be coupled with ${ }^{13} \mathrm{C}$ experiments, which have yet to be explored.

Table 5.6: Summary of results for diazomethane-modified synthetic peptides followed by elution with $\mathrm{Et}_{3} \mathrm{~N}^{+} \mathrm{H}^{-} \mathrm{OAC}$

\begin{tabular}{|c|c|c|c|c|c|c|c|c|c|}
\hline \multirow{2}{*}{ Peptide } & \multicolumn{2}{|c|}{ Unmodified } & \multicolumn{2}{|c|}{ Modified mass } & \multicolumn{2}{c|}{$\Delta \mathrm{m}$} & \multicolumn{3}{c|}{ Methylation } \\
\cline { 2 - 10 } & $\mathrm{m} / \mathrm{z}$ & $\begin{array}{c}\text { neutral } \\
\text { mass }\end{array}$ & $\mathrm{m} / \mathrm{z}$ & $\mathrm{Da}$ & $\mathrm{m} / \mathrm{z}$ & $\mathrm{Da}$ & $\begin{array}{c}\% \mathrm{~N}- \\
\text { terminus }\end{array}$ & $\begin{array}{c}\text { \# Me } \\
\text { groups }\end{array}$ & $\begin{array}{c}\text { \# fixed } \\
\text { charges }\end{array}$ \\
\hline [FLEEVK] $^{2+}$ & 382.7 & 763.4 & 445.7 & 891.4 & 63 & 128 & 100 & 9 & 2 \\
\hline [FLEEVR] $^{2+}$ & 396.7 & 791.4 & 438.7 & 877.4 & 42 & 86 & 100 & 6 & 1 \\
\hline [FLEENK] $^{2+}$ & 390.2 & 778.4 & 453.2 & 906.4 & 63 & 128 & 100 & 9 & 2 \\
\hline [FLEEYK] $^{2+}$ & 414.7 & 827.4 & 484.7 & 969.4 & 70 & 142 & 100 & 10 & 2 \\
\hline
\end{tabular}

* - percentage of $\mathrm{N}$-terminal amine quaternization 


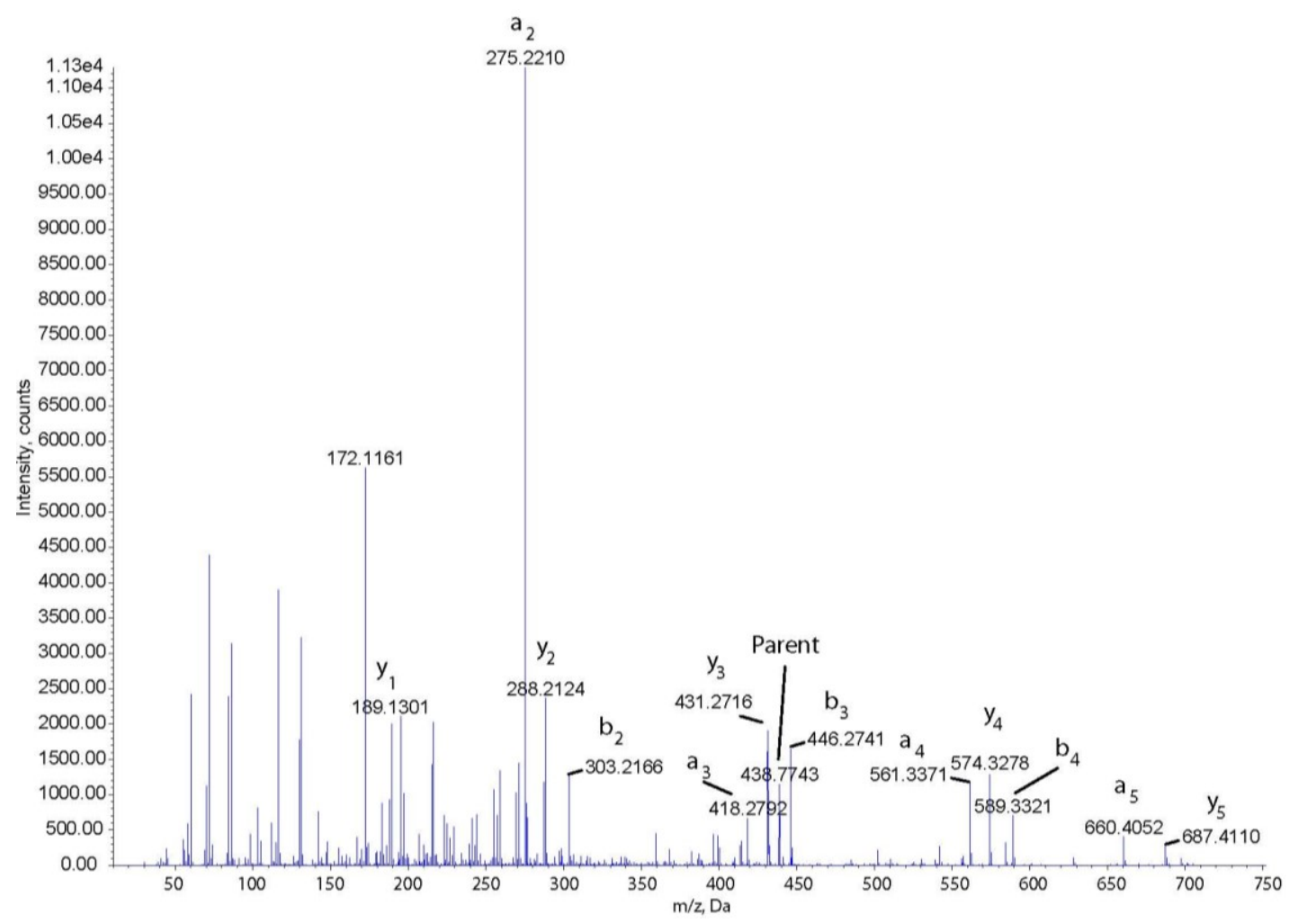

Figure 5.25: $M S^{2}$ results of fully modified FLEEVR ( $\left.\mathrm{m} / \mathrm{z} 438.7\right)$ with methyl esterification.

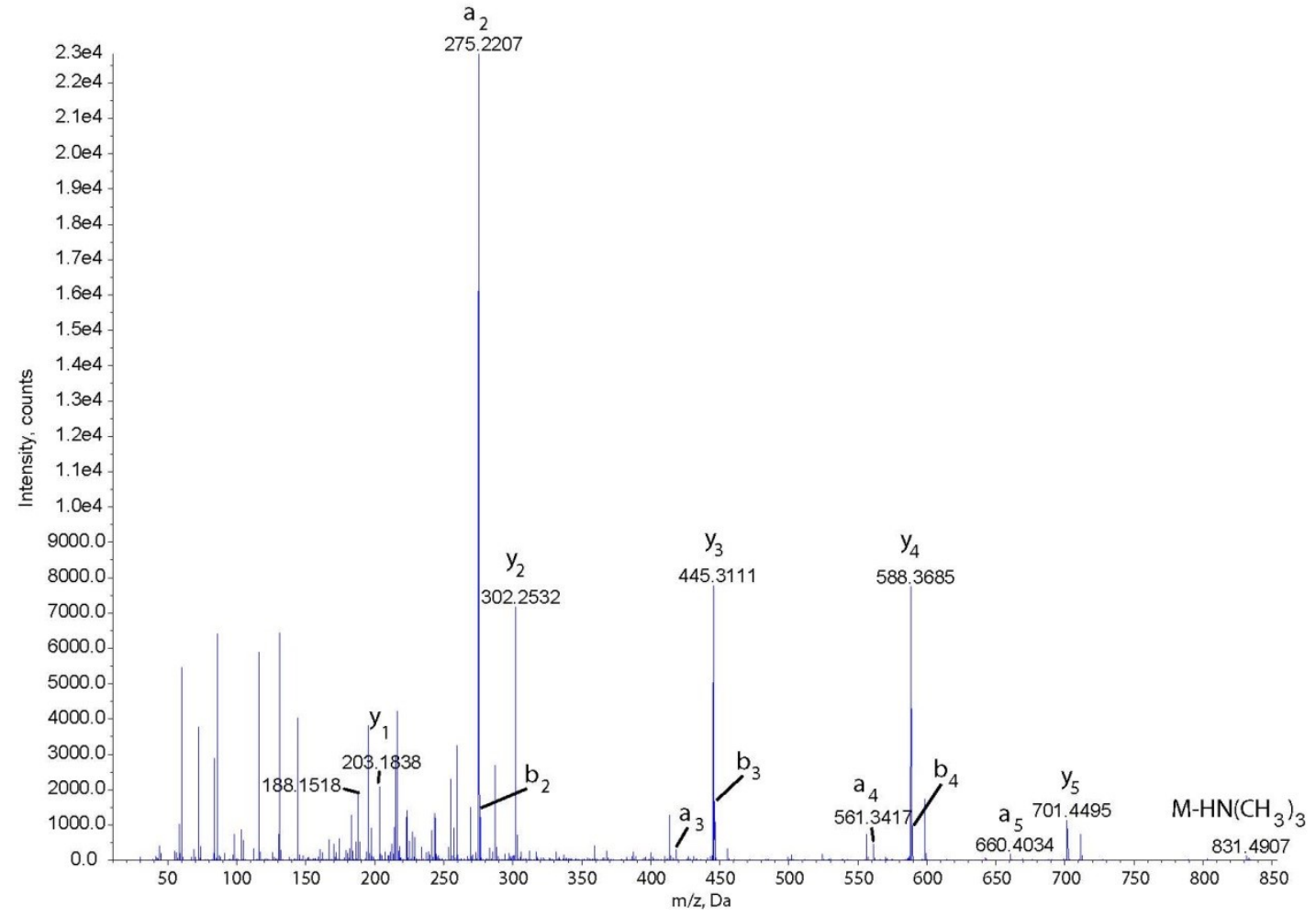

Figure 5.26: $\mathrm{MS}^{2}$ results of fully modified FLEEVK $(\mathrm{m} / \mathrm{z} 445.3)$ with methyl esterification. 


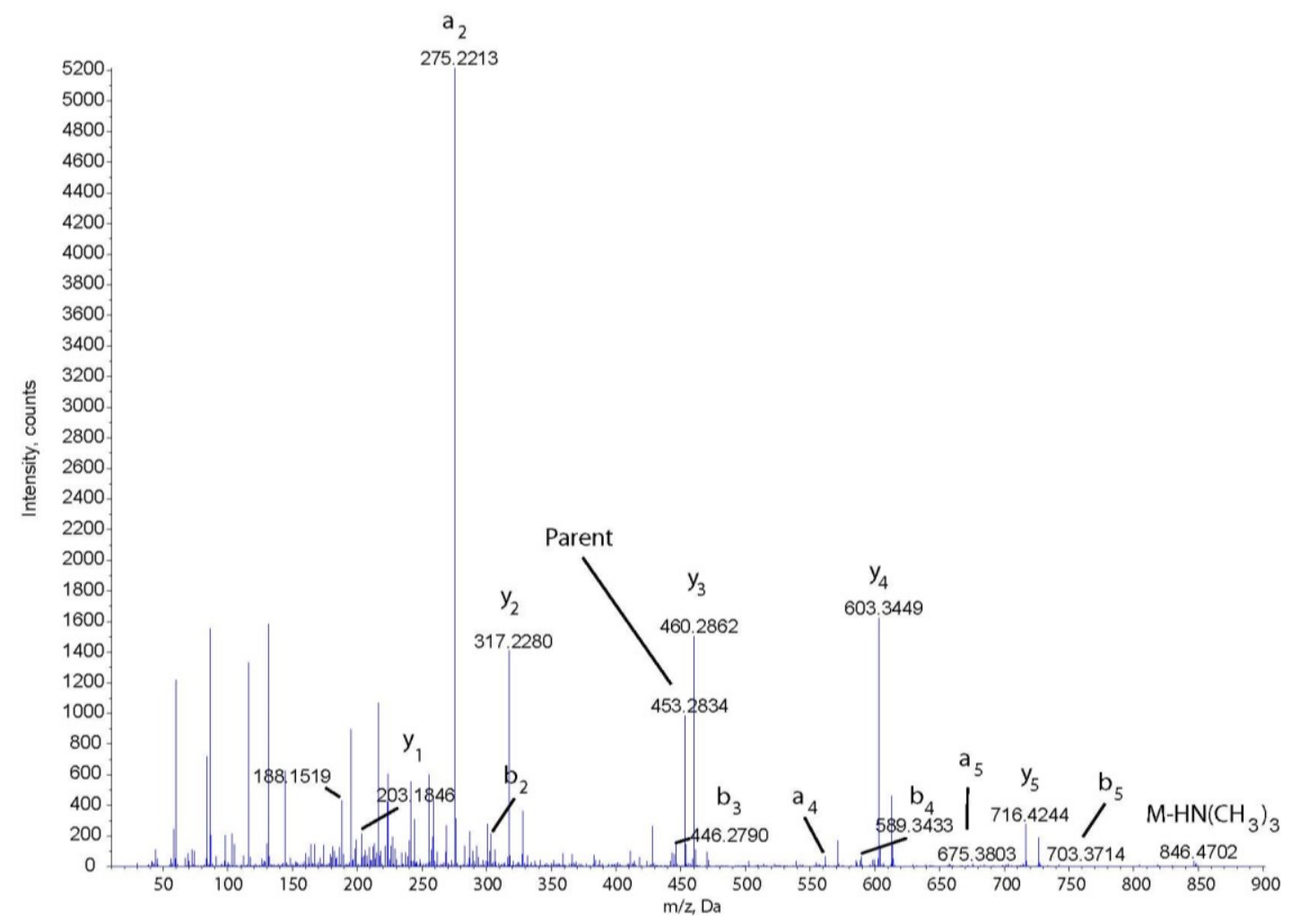

Figure 5.27: $\mathrm{MS}^{2}$ results of fully modified FLEENK (m/z 453.2) with methyl esterification.

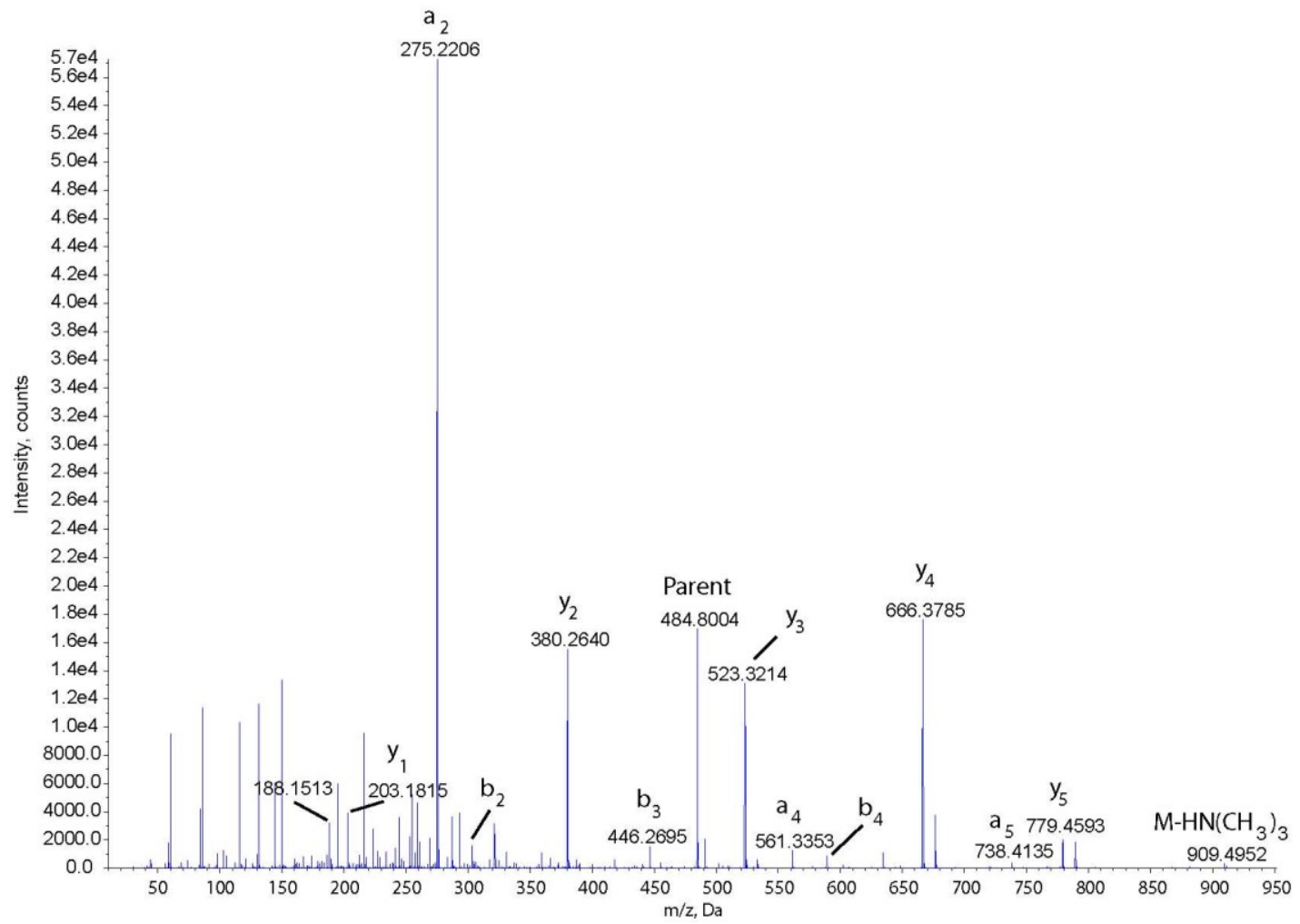

Figure 5.28: $\mathrm{MS}^{2}$ results of fully modified FLEEYK $(\mathrm{m} / \mathrm{z} 484.8)$ with methyl esterification. 


\subsection{On-column derivatization of lipids}

On-column derivatization of lipids varies slightly from peptides. A brief outline of the procedure is shown below. A fritted $18 \mathrm{~cm}$ long piece of fused silica (200 $\mu \mathrm{m}$ ID) was packed with previously washed and conditioned PolySULFOETHYL A SCX resin using a high pressure vessel. Following the potassium phosphate buffer wash, a solution containing approximately 7 $\mu g$ of PE lipid was prepared with $30 \mu \mathrm{L}$ of ethanol and $70 \mu \mathrm{L}$ of $50 \mathrm{mM} \mathrm{H} \mathrm{H}_{3} \mathrm{PO}_{4}$ before derivatization with diazomethane. PC lipid required ethanol and $0.5 \mu \mathrm{L}$ of a 1:5 $\mathrm{HBF}_{4}$ : ether solution prior to derivatization. Bound analytes were allowed to dry using nitrogen gas. Approximately $250 \mu \mathrm{L}$ of ethereal diazomethane was added to the HPV and was allowed to flow through the column. Once dried, a $200 \mathrm{mmol}$ solution of ammonium bicarbonate in ethanol was passed through the column to elute the analytes from the column. Sample was analyzed directly via mass spectrometry on the Qstar without prior desalting cleanup to analysis.

On-column TrEnDi was optimized to allow trimethylation of lipid primary amine groups as well as methylation of PE and PC lipid phosphate groups. The strategy, as with solution based TrEnDi, creates a fixed positive charge on the primary amine of PE sn-3 groups while also neutralizing the negative charge of the phosphate group of PC and PE lipids at higher $\mathrm{pH}$ thereby eliminating the need for protonation. Figure 5.29 to Figure 5.32 demonstrate that oncolumn TrEnDi is equally as efficient as in solution based TrEnDi for both PC and PE lipids. MS analysis of PE lipids demonstrated a five-fold enhancement in sensitivity in signal of the modified analyte when an equimolar mixture of modified and non-modified PE was analyzed 
(Figure 5.33). Sensitivity test was not performed on-column for PC lipids. As previously mentioned in chapter 4, fragmentation of the TrEnDi-modified lipids often revealed one predominant fragmentation pathway, permitting sensitive precursor ion analyses. More optimization is required to fully methylate PS and SM successfully on-column.

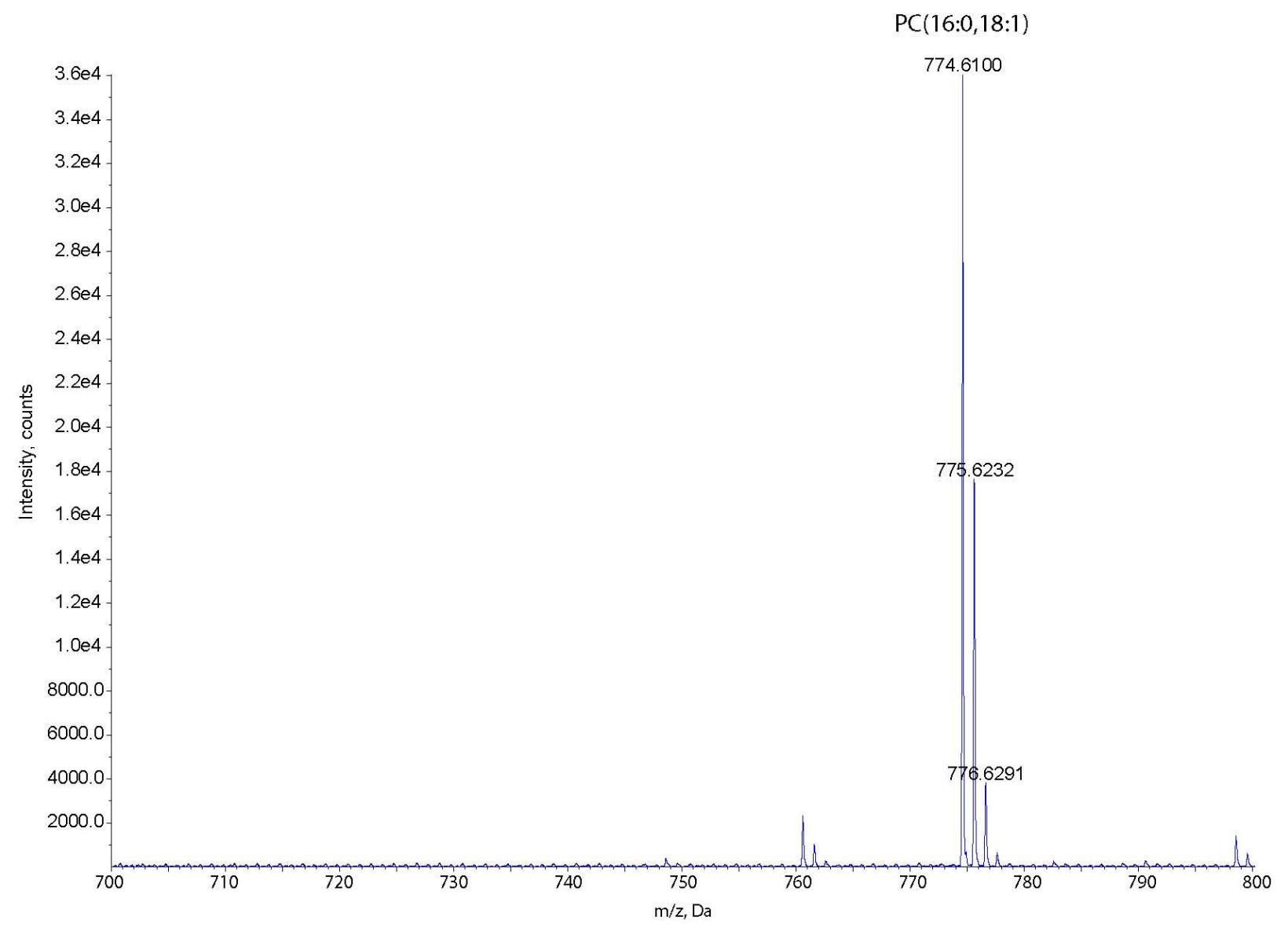

Figure 5.29: PC (16:0, 18:1) modified via on-column TrEnDi 


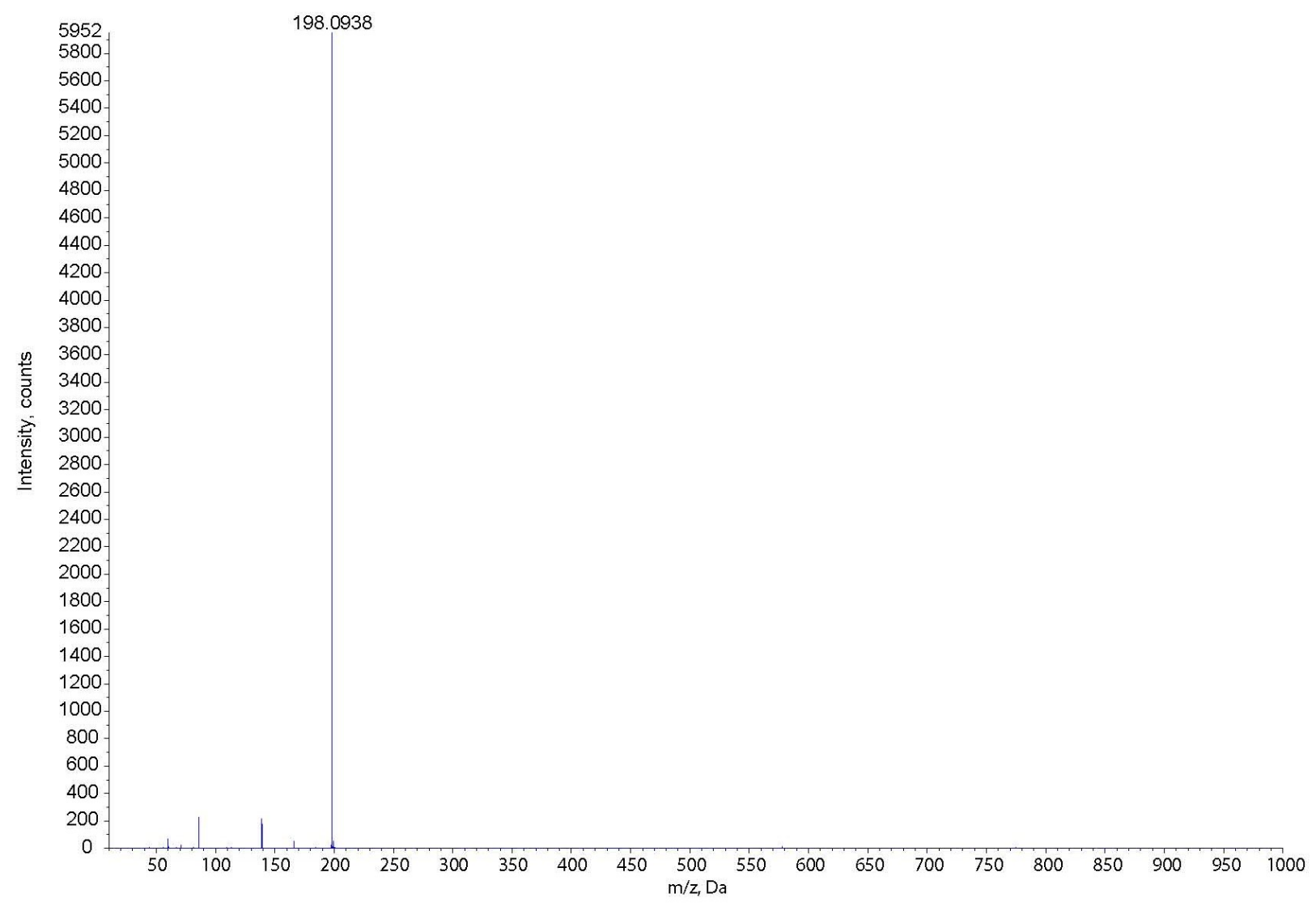

Figure 5.30: $\mathrm{MS}^{2}$ of fully modified PC $(16: 0,18: 1)$ with one dominant fragmentation channel. 


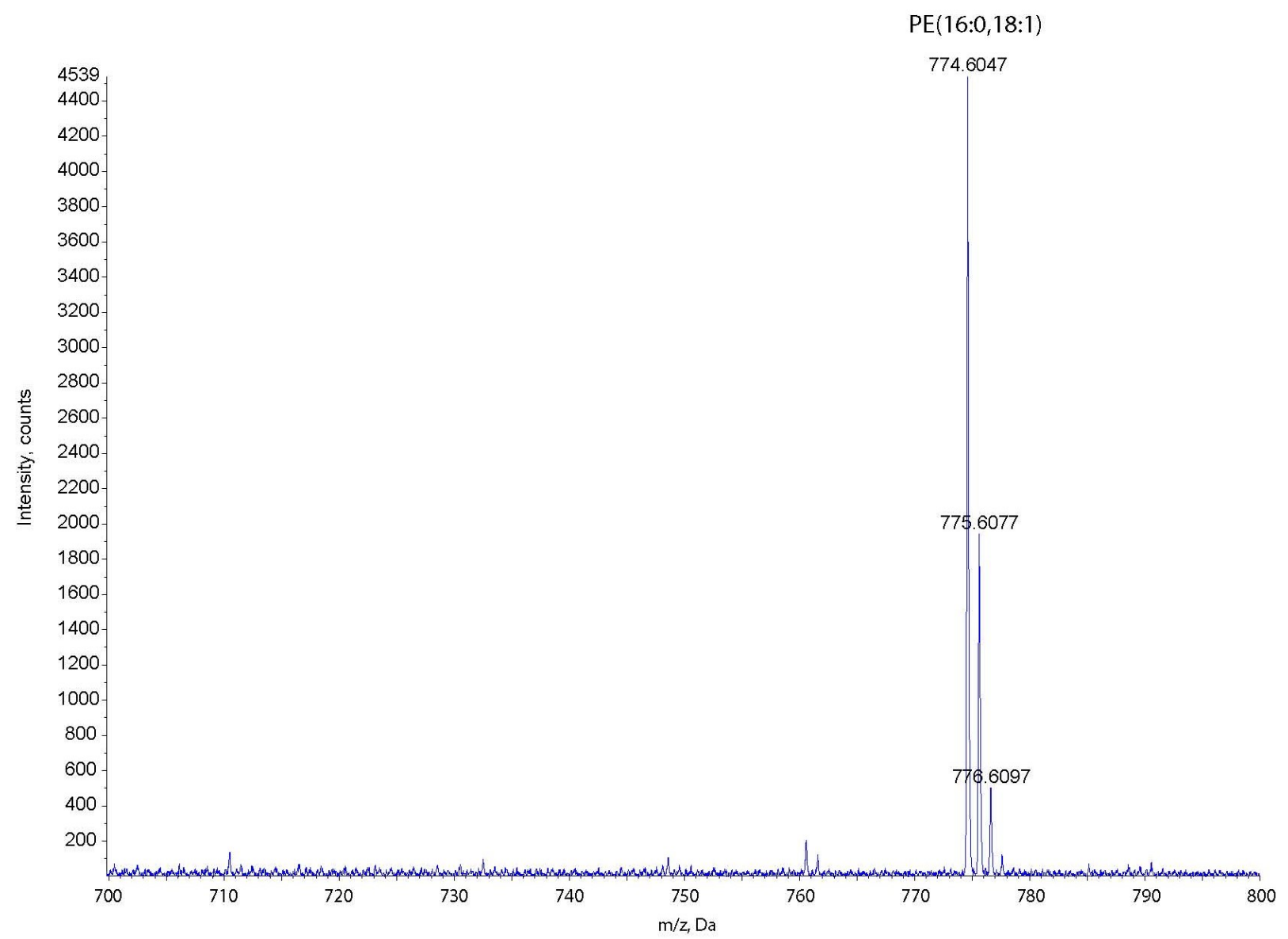

Figure 5.31: PE (16:0, 18:1) modified via on-column TrEnDi 


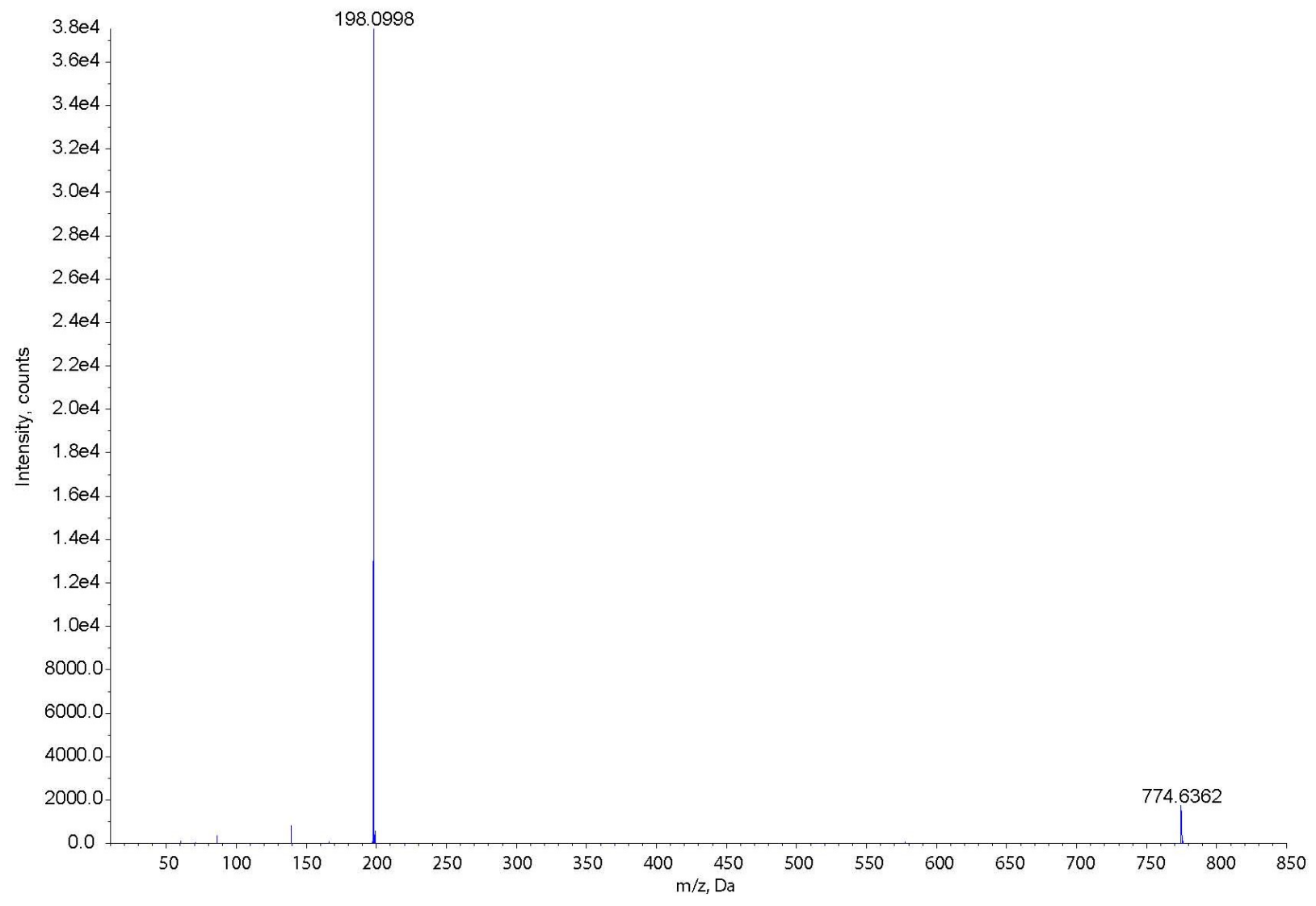

Figure 5.32: $\mathrm{MS}^{2}$ of fully modified PE $(16: 0,18: 1)$ with one dominant fragmentation channel. 


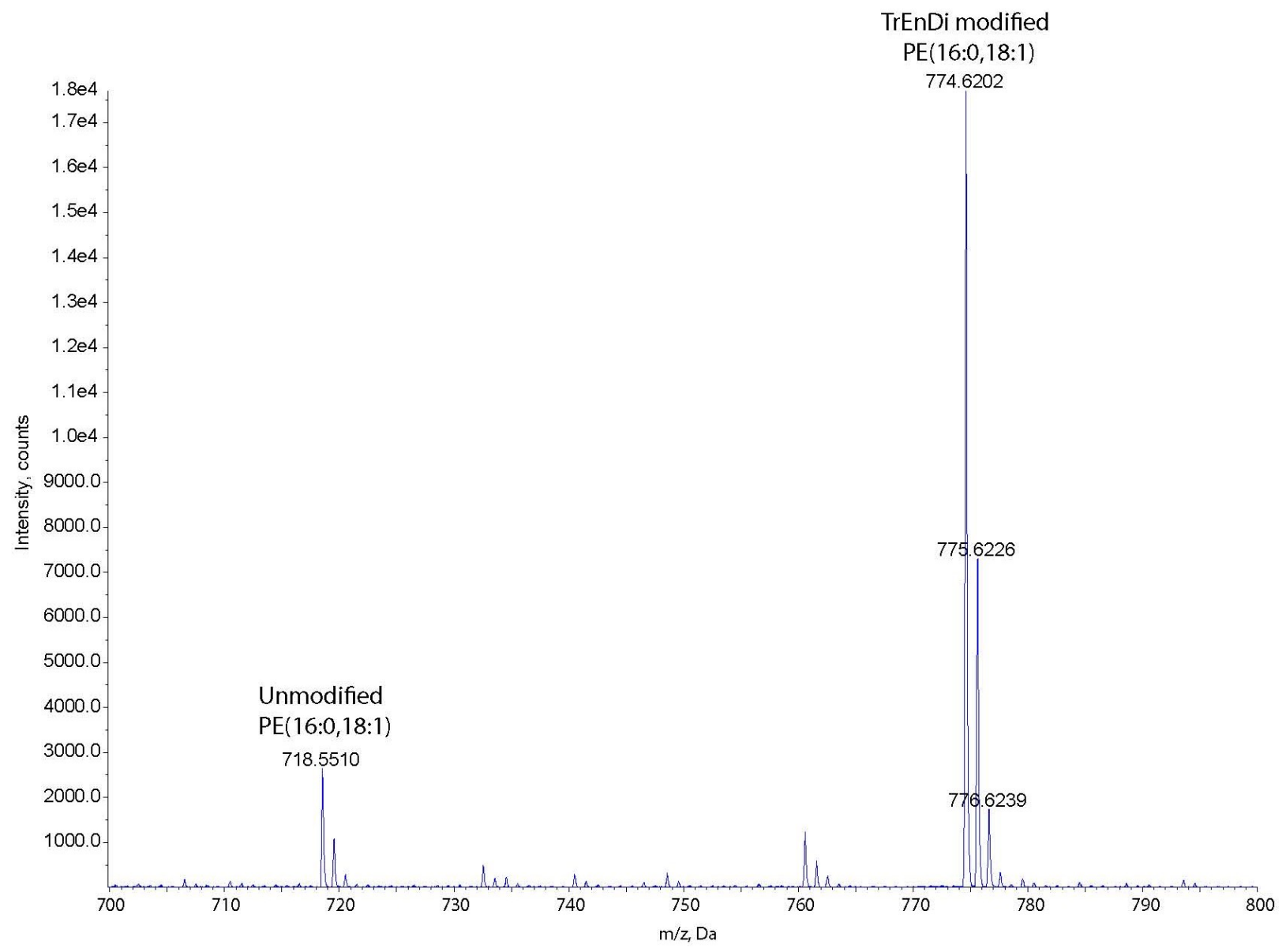

Figure 5.33: Sensitivity test on equimolar amounts of unmodified vs. TrEnDi modified PE $(16: 0,18: 1)$

\section{$5.5^{13} \mathrm{C}$ labelling of lipids}

TrEnDi's ability to fully methylate lipids such that they bear fixed, permanent positive charges allowing them to be easily ionized in the gas phase has been established. There is also the advantage for an increase in sensitivity following methylation due to the characteristic fragmentation channels of the fully modified lipid. However, phosphatidylethanolamine and phosphatidylcholine lipids would be identical post TrEnDi modification if they had the same 
chain lengths. For example, a PC $(16: 0,18: 1)$ with an $\mathrm{m} / \mathrm{z}$ of 760 and a PE $(16: 0,18: 1)$ with an $\mathrm{m} / \mathrm{z}$ of 718 would have the same chemical properties and the same mass of 774 post TrEnDi modification (see Figure 5.29 and Figure 5.31 in section 5.4).

The inability to distinguish between these two classes of lipids is not favourable. However, performing TrEnDi modification using ${ }^{13} \mathrm{C}$ diazomethane ${ }^{123}$ would allow one to distinguish the differences between both species via mass spectrometry (Figure 5.34 and Figure 5.35).

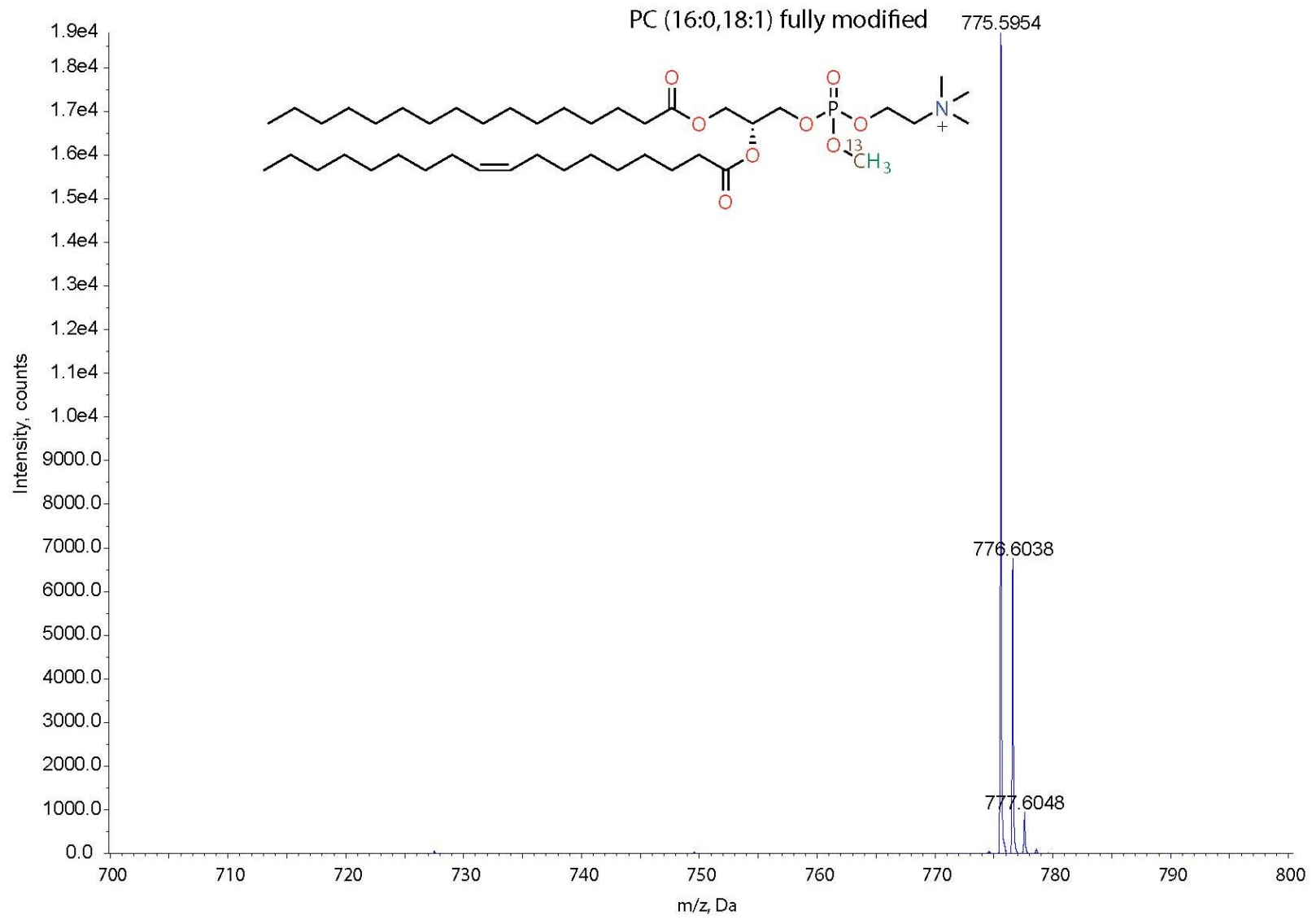

Figure 5.34: $\mathrm{MS}$ analysis of $\mathrm{PC}(16: 0,18: 1)$ fully modified with ${ }^{13} \mathrm{C}$ diazomethane 


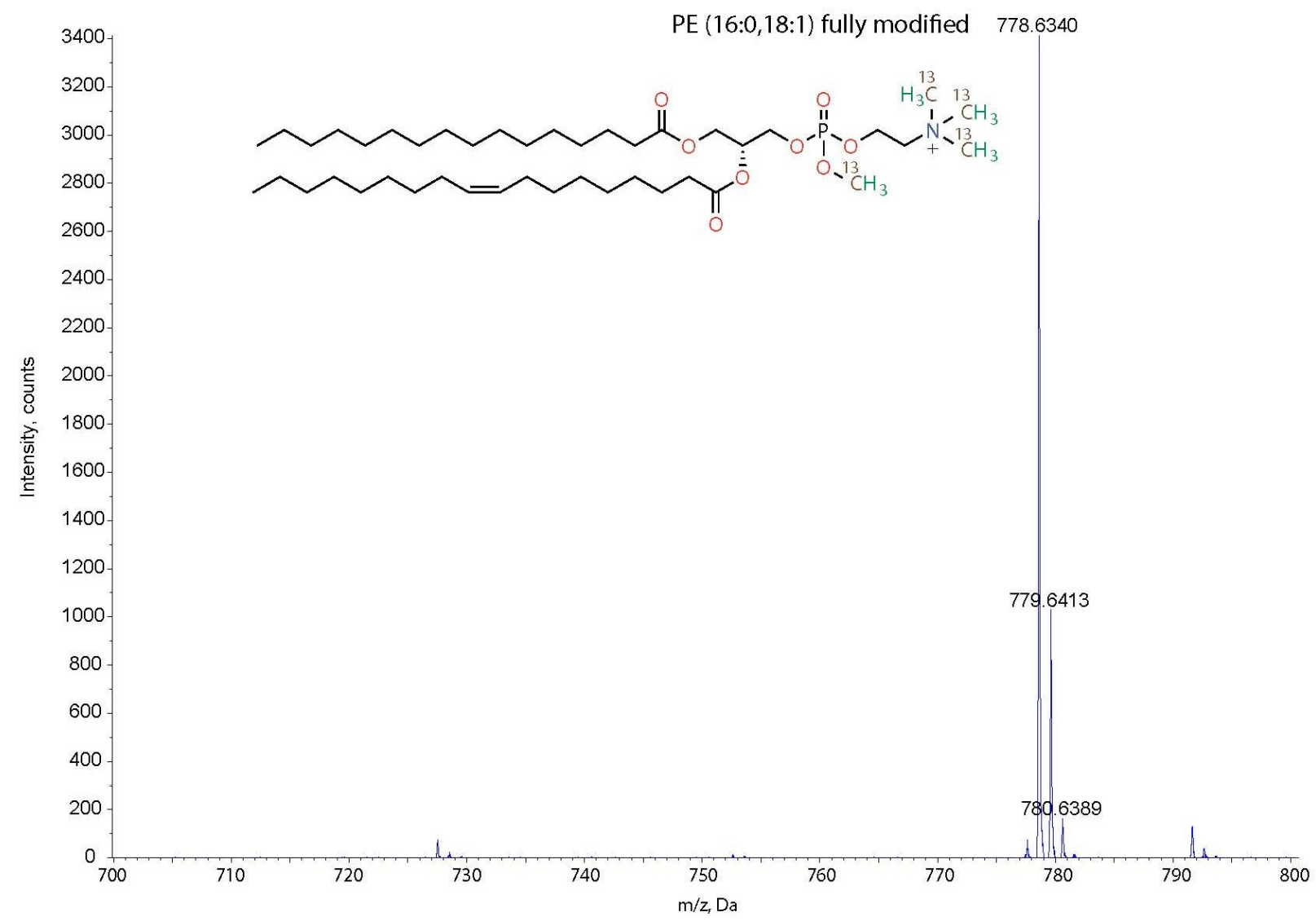

Figure 5.35: MS analysis of PE $(16: 0,18: 1)$ fully modified with ${ }^{13} \mathrm{C}$ diazomethane

Once fully modified with ${ }^{13} \mathrm{C}$ diazomethane, the mass shifts from 774 to 775 and $778 \mathrm{Da}$ for PC and PE respectively can easily be seen on the mass spectrum of these two lipids producing equally sensitive signal for equal molar amounts of starting material (Figure 5.36). 


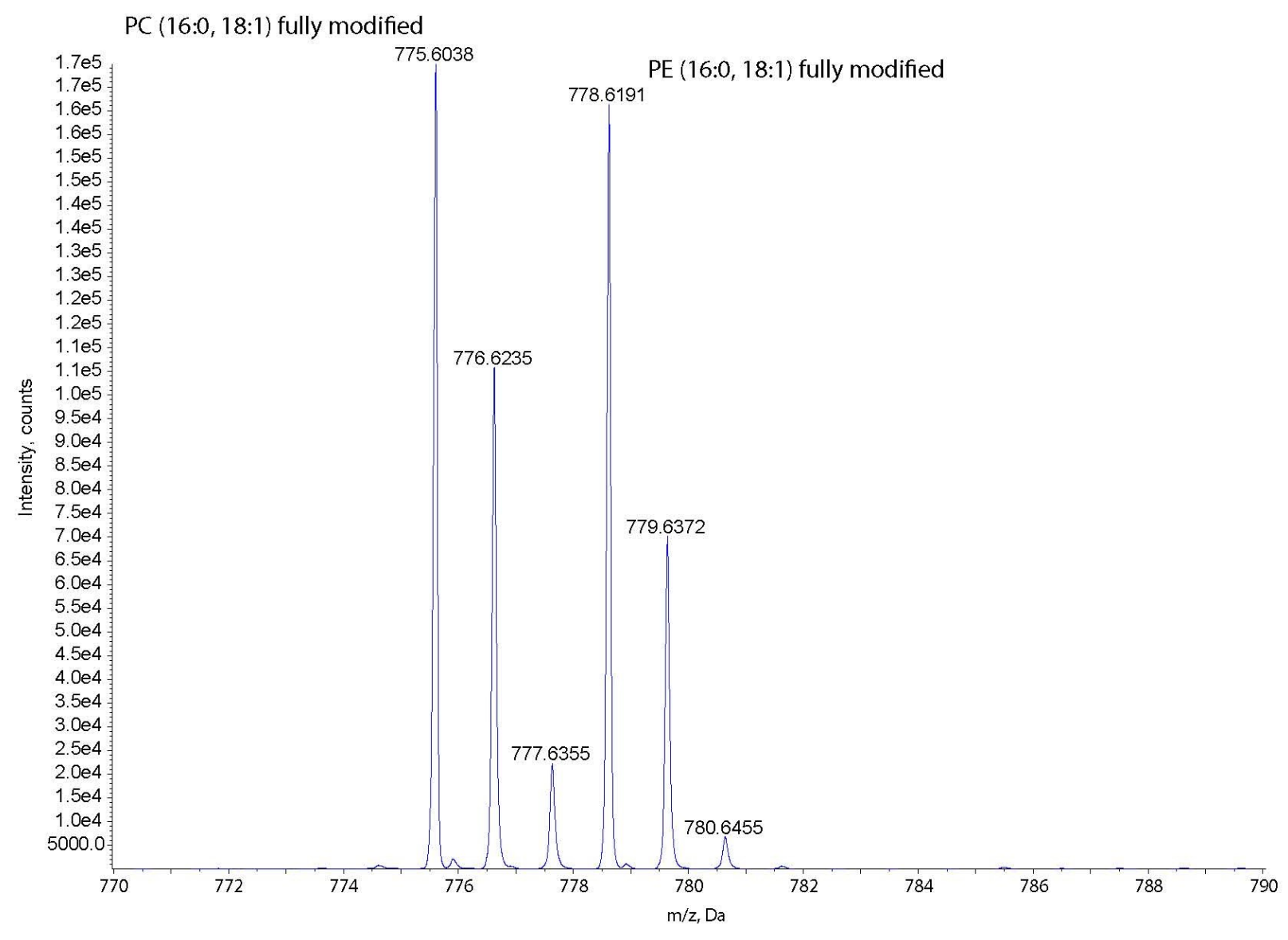

Figure 5.36: MS analysis of both PC and PE (16:0, 18:1) post TrEnDi modification with ${ }^{13} \mathrm{C}$ diazomethane. The three Da mass shift between both species allows for easy identification.

Furthermore, we see that the dominant fragment ion also contains the characteristic shifts from 198 to 199 and 202 for both PC and PE respectively (Figure 5.37 and Figure 5.38) 


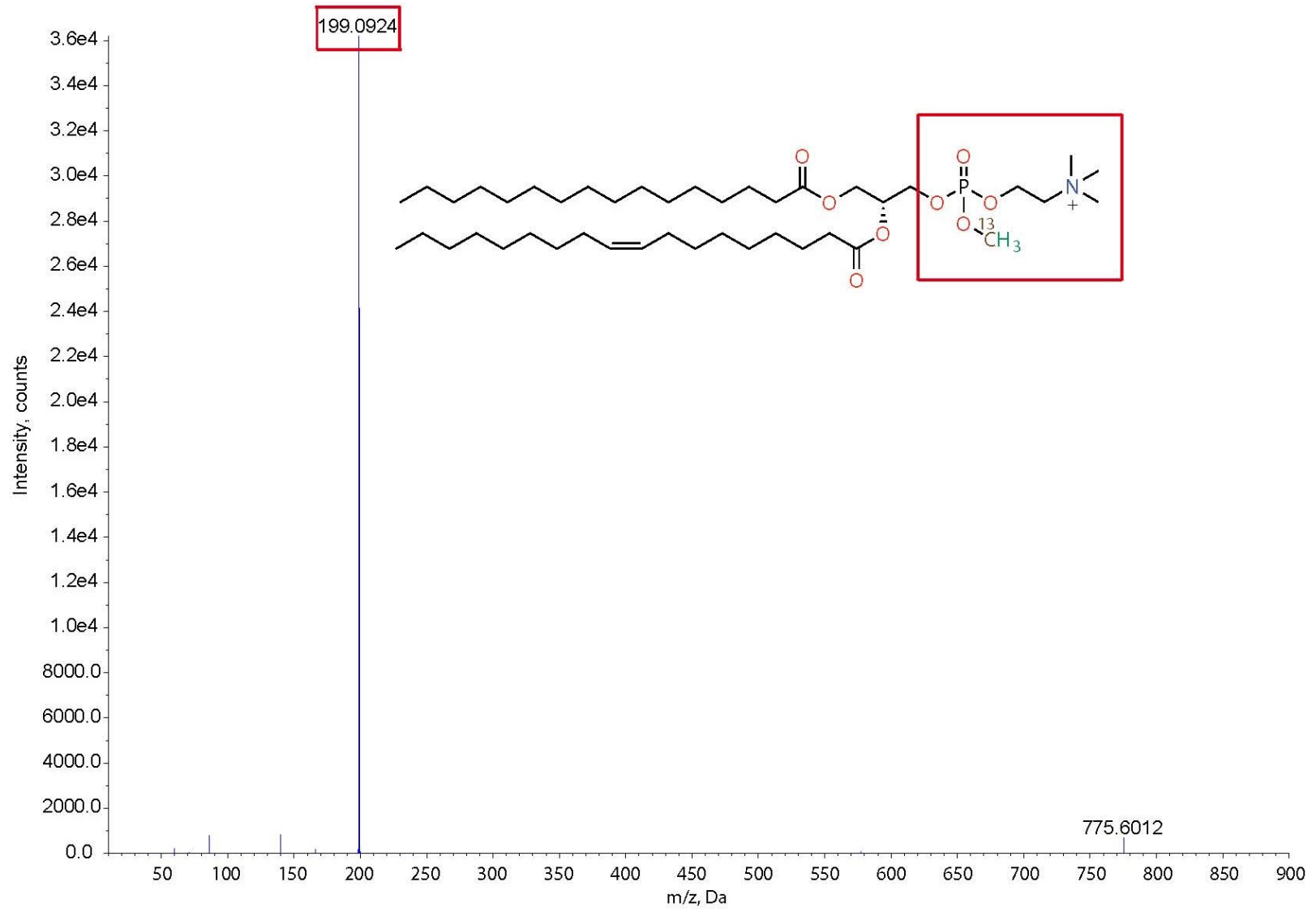

Figure 5.37: $\mathrm{MS}^{2}$ analysis of $\mathrm{m} / \mathrm{z} 775.6$ fully modified $\mathrm{PC}(16: 0,18: 1)$ with ${ }^{13} \mathrm{C}$ diazomethane 


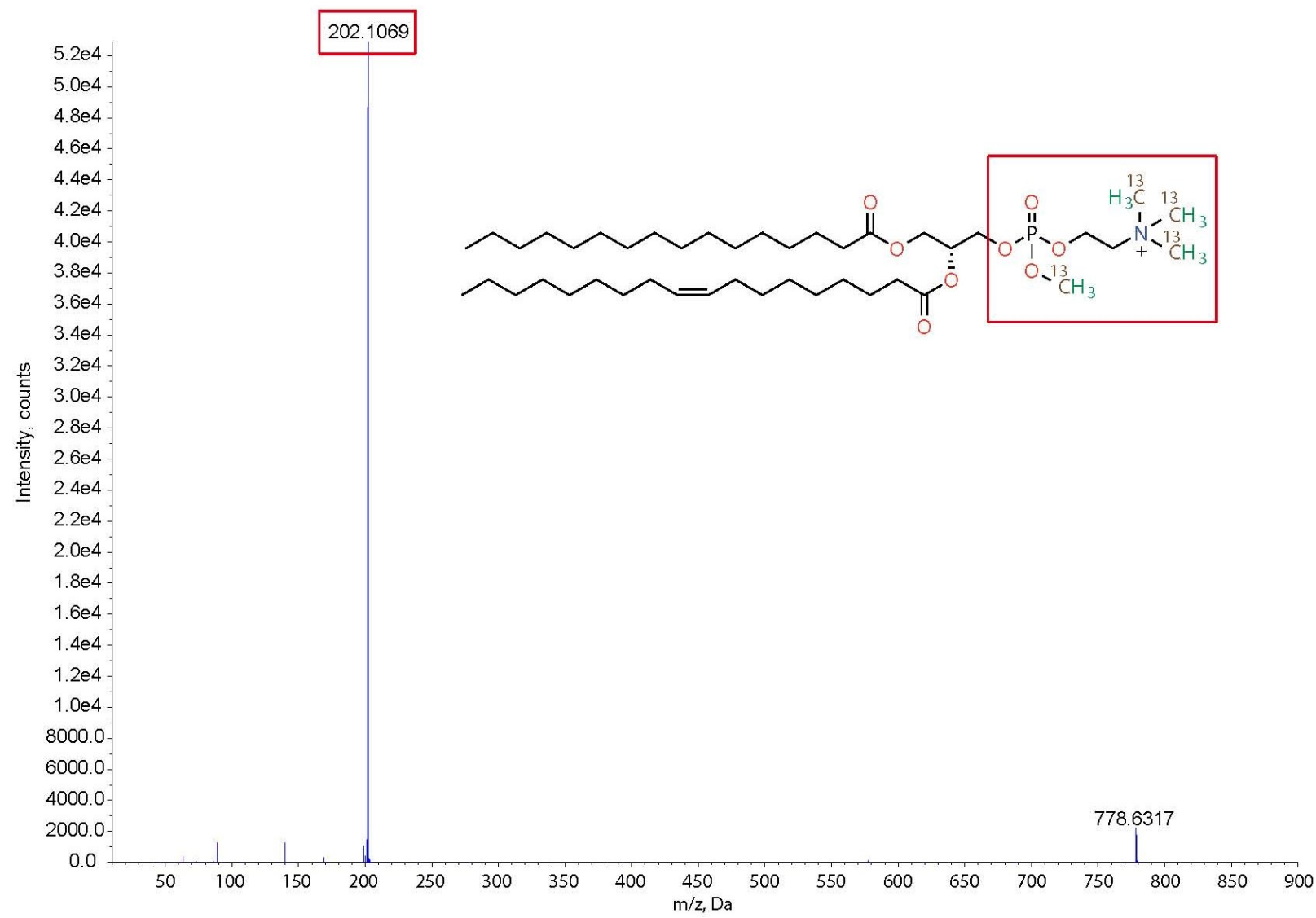

Figure 5.38: $\mathrm{MS}^{2}$ analysis of $\mathrm{m} / \mathrm{z} 778.6$ fully modified $\mathrm{PE}(16: 0,18: 1)$ with ${ }^{13} \mathrm{C}$ diazomethane

When performing HPLC separation, we see that both TrEnDi modified PC and PE of the same chain lengths are eluted at the same time as expected. However, using the precursor ion scan for ions 199 and 202, we can easily distinguish between these two classes of lipids, despite having the same molecular formula (Figure 5.39). ${ }^{13} \mathrm{C}$ versus ${ }^{12} \mathrm{C}$ diazomethane studies can be applied to large scale analyses to help determine the number of methylations. Large scale studies using this technique have yet to be explored. 

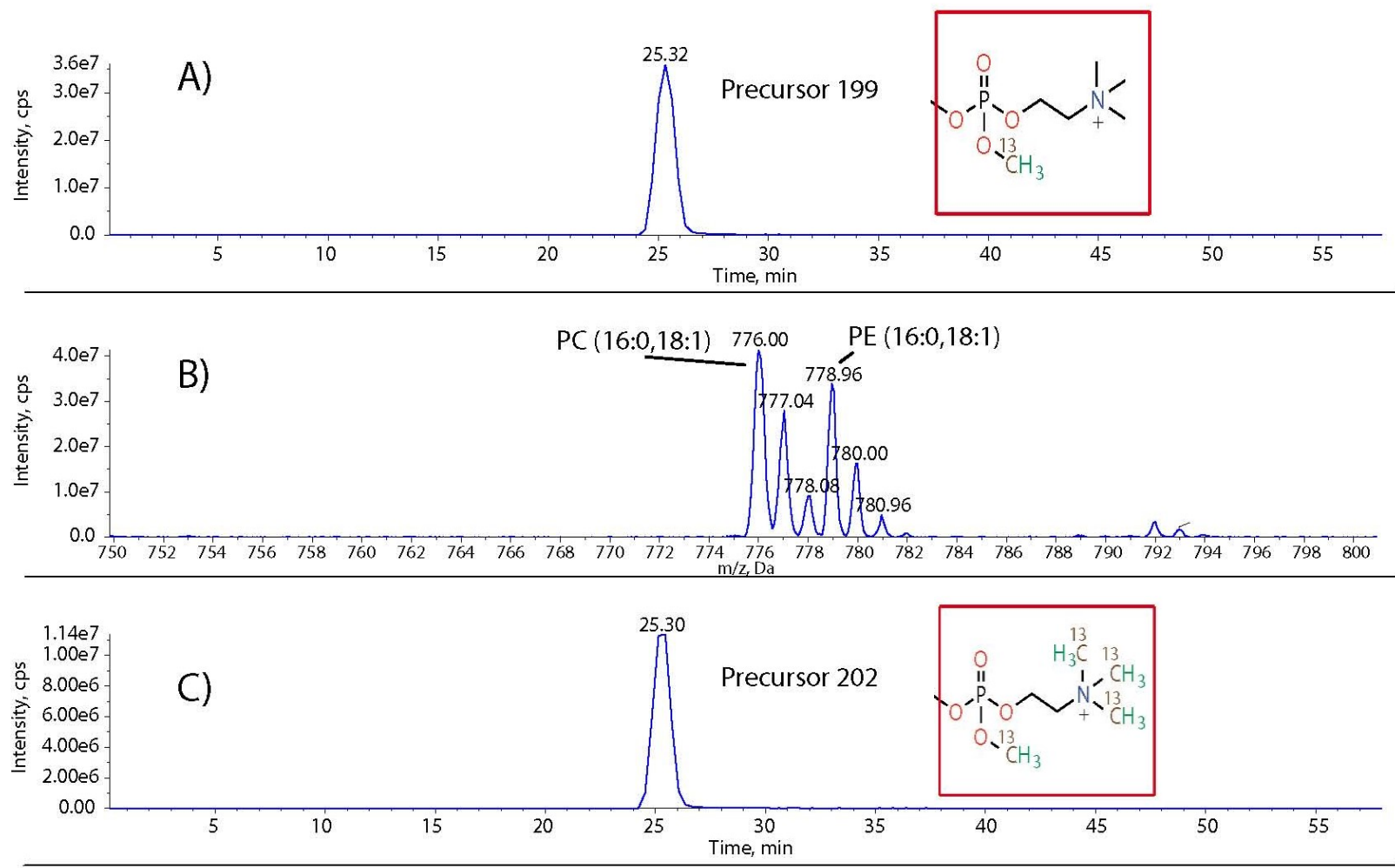

Figure 5.39: A) Precursor ion scan for $\mathrm{m} / \mathrm{z}$ 199, characteristic fragment of $P C(16: 0,18: 1)$ fully modified with ${ }^{13} \mathrm{C}$ diazomethane; B) MS analysis on the Qtrap of both PC and PE $(16: 0,18: 1)$ fully modified with ${ }^{13} \mathrm{C}$ diazomethane; C) Precursor ion scan for $\mathrm{m} / \mathrm{z} 202$, characteristic fragment of PE (16:0, 18:1) fully modified with ${ }^{13} \mathrm{C}$ diazomethane. 


\subsection{Conclusion}

The trimethylation enhancement using diazomethane (TrEnDi) technique has been successfully developed on small peptides and various lipids and shows promising potential for the identification of phosphopeptides and full proteins. Methylation has been shown to occur on functional groups with pKa values lower than 11 and produce quaternary ammonium groups on lysine side chains, peptide $\mathrm{N}$-termini, lipid primary amines and imidazole groups. Phenol groups and phosphate groups were also shown to be methylated (without adding a fixed positive charge); however, amide and guanidinium groups were not methylated by this strategy. All carboxylic acids were methylated using this chemistry and methods have been developed to keep the methyl esters or to successfully revert them back to acids with the use of a base.

TrEnDi modifies peptides and lipids such that they contain fixed, permanent positive charges, increasing the sensitivity of ESI MS analysis by driving the charge state of each analyte to a single value and opening the possibility of using aprotic solvents. The derivatization chemistry for both lipids and peptides is predictable but equally important is that the fragmentation behaviour of the TrEnDi-modified analytes is also predictable. TrEnDi-modified peptides demonstrate a propensity to fragment and form the $a_{2}$ fragment ion. In doubly charged ions, the $a_{2}$ fragment was the most intense fragment revealing how this chemistry is able to enhance the sensitivity of MRM analyses. The proof of this sensitivity gain in peptides was observed in the analysis of an unobserved peptide from BSA using conventional proteomics 
methods. TrEnDi places a fixed positive charge on peptides and induces predictable fragmentation behaviour, permitting "blind" MRM experiments and providing a mechanism to observe and quantify elusive peptides.

TrEnDi-modified lipids produce a permanently singly charged lipid species that demonstrate a propensity to fragment and form a positively charged polar head group. These polar head groups were the most intense fragments revealing how this chemistry is able to enhance the sensitivity of precursor ion scanning analyses. The proof of this sensitivity gain was observed in the comparison of equimolar amounts of unmodified and TrEnDi-modified lipids using conventional lipidomics methods. TrEnDi places a fixed positive charge on lipids and induces anticipated head group fragmentation behaviour, permitting highly sensitive precursor ion scanning experiments and providing a mechanism to observe and quantify elusive lipids.

A preliminary study using triethylammonium acetate as an elution solution indicates successful and complete methylation of peptides without base hydrolysis of the methyl esters. This provides some promising features for full protein analysis as it may eliminate the need for salt cleanup and may increase the hydrophobicity of fully modified peptides. In addition to protein studies, this new elution technique could prove to be useful for further phosphoproteomic studies as the phosphate groups would remain fully modified, hence neutralizing their negative charge while providing the benefits of TrEnDi to the rest of the peptide.

Furthermore, TrEnDi has shown the ability to incorporate stable isotopes in both peptides and lipids. This now offers another means for quantitative analysis, such that two of 
the same analytes from different samples can be analyzed together simultaneously and the relative difference in their intensities can be measured. 


\section{References:}

${ }^{1}$ Lander, E.S. et al. Initial sequencing and analysis of the human genome. Nature 409, 860-921 (2001)

${ }^{2}$ Economic Impact of the Human Genome Project-Battelle http://battelle.org/docs/default-document-

library/economic impact of the human genome project.pdf Retrieved, November $30^{\text {th }} 2013$.

${ }^{3}$ Lander, E.S. Initial impact of the sequencing of the human genome. Nature 470, 187-197

(2011)

${ }^{4}$ Nobel Prize 2002. http://nobelprize.org/nobel_prizes/chemistry/laureates/2002/

${ }^{5}$ Fenn, J.B., Mann, M., Meng, C.K., Wong, S.F. \& Whitehouse, C.M. Electrospray ionization for mass spectrometry of large biomolecules. Science 246, 64 (1989)

${ }^{6}$ Tanaka, K. et al. Protein and polymer analyses up to m/z 100000 by laser ionization time-offlight mass spectrometry. Rapid Commun Mass Spectrom 2, 151-153 (1988)

${ }^{7}$ Ong, S. E., Mann, M. Nature chemical biology 1, 252-262 (2005)

${ }^{8}$ Bou Khalil, M., Hou, M., Zhou, H., Elisma, F., Swayne, L. A., Blanchard, A. P., Figeys, D. Lipidomics Era: Accomplishments and Challenges. Mass Spectrometry Reviews, 29, 877-929. (2010)

${ }^{9}$ Byrdwell, W.C. Atmospheric pressure chemical ionization mass spectrometry for analysis of lipids. Lipids 36, 327-346 (2001)

${ }^{10}$ Byrdwell, W.C. The bottom-up solution to the triacylglycerol lipidome using atmospheric pressure chemical ionization mass spectrometry. Lipids 40, 383-417 (2005)

${ }^{11}$ Pandor, A., Eastham, J., Beverley, C., Chilcott, J., Paisley, S. Health technology assessment. 8, 1-121 (2004)

${ }^{12}$ Smith, J. C., Figeys, D. Biochemistry and cell biology = Biochimie et biologie cellulaire 86, 137148 (2008)

${ }^{13}$ Buhimschi, C. S., Weiner, C. P., Buhimschi, I. A. Obstetrical \& gynecological survey 61, 481-486 (2006)

${ }^{14}$ Liu, X., Wen, F., Yang, J., Chen, L., Wei, Y. Q. Mass spectrometry reviews 29, 197-246 (2010)

${ }^{15}$ Bouchal, P., Dvorakova, M., Scherl, A., Garbis, S. D., Nenutil, R., Vojtesek, B. Proteomics 13, 1053-108 (2013)

${ }^{16}$ Diamandis, E. P. Molecular \& cellular proteomics : MCP 3, 367-378 (2004)

${ }^{17}$ Kolch, W., Neususs, C., Pelzing, M., Mischak, H. Mass spectrometry reviews 24, 959-977 (2005)

18 Bensimon, A., Heck, A. J., Aebersold, R. Annu Rev Biochem 81, 379-405 (2012)

${ }^{19}$ Wenk, M. The emerging field of lipidomics. Nature 4, 594-610 (2005)

${ }^{20}$ Crick, F. Central Dogma of Molecular Biology. Nature 227, 561-563 (1970)

${ }^{21}$ Voet, D., Voet, J.G., Pratt, C.W. Fundamentals of Biochemistry $3^{\text {rd }}$ Edition. Wiley and Sons 74 (2008)

${ }^{22}$ Sigma Aldrich http://www.sigmaaldrich.com/life-science/metabolomics/learningcenter/amino-acid-reference-chart.html

${ }^{23}$ The University of Queensland http://di.uq.edu.au/sparq/images/aastructures.jpg 
${ }^{24}$ Wikimedia Commons.

http://upload.wikimedia.org/wikipedia/commons/c/c9/Main_protein_structure_levels_en.svg

${ }^{25}$ UniProt. UniProt Knowledgebase: Controlled vocabulary of posttranslational modifications.

${ }^{26}$ Cohen, $\mathrm{P}$. The role of protein phosphorylation in human health and disease. Eur. J. Biochem.

268, 5001-5010 (2001)

${ }^{27}$ Hunter, T., Sefton, B.M. Transforming gene product of Rous sarcoma virus phosphorylates tyrosine. Proc Natl Acad Sci USA 77, 1311-1315 (1980)

${ }^{28}$ Jackson, S.N., Wang, H.Y.J., Woods, A.S., In situ structural characterization of glycerophospholipids and sulfatides in brain tissue using MALDI-MS/MS. J Am Soc Mass

Spectrom 18, 17-26 (2007)

${ }^{29}$ Agranoff, B.W. et al. In basic neurochemistry molecular, cellular, and medical aspects, 6th ed. Lippincott Williams and Wilkins: Philadelphia. 47-67 (1999)

${ }^{30}$ Hannun, Y.A., Obeid, L.M. Principles of bioactive lipid signalling: lessons from sphingolipids. Nature Reviews Moll Cell Bio 9, 139-150 (2008)

${ }^{31}$ Butterfield, D.A., Lauderback, C.M. Lipid peroxidation and protein oxidation in Alzheimer's disease brain. Free Radical Bio Med 32, 1050-1060 (2002)

32 Piomelli, D., Astarita, G., Rapaka, R. A neuroscientist's guide to lipidomics. Nat Rev Neurosci 8,743-754 (2007)

${ }^{33}$ NASA. http://mars.nasa.gov/msl/mission/instruments/spectrometers/sam/ . Accessed December $2^{\text {nd }} 2013$.

${ }^{34}$ Miller, P.E., Denton, M.B. The quadrupole mass filter: Basic operating concepts. J Chem Educ 63, 617-623 (1986)

${ }^{35}$ Dole, M., et al. Molecular beams of macroions. J Chem Phys 49, 2240 (1968)

${ }^{36}$ Smith, J.C. Mass Spectrometry-Based Proteomics: Non-Covalent Interactions and Protein Identification. Ph.D. Thesis, York University, Toronto, Ont., April 2005.

${ }^{37}$ Vestal, M.L. Chem Rev, 2001, 101, 361-375

${ }^{38}$ Taylor, G.I. The stability of horizontal fluid interface in a vertical electric field. J Fluid Mech 2, 1-15 (1965)

${ }^{39}$ Steen, H., Mann, M. Nat Rev Mol Cell Bio, 2004, 5, 699-711

${ }^{40}$ Gale, D.C., Smith, R.D. Small volume and low flow-rate electrospray ionization mass spectrometry of aqueous samples. Rapid Commun Mass Spectrom 7, 1017-1021 (1993)

${ }^{41}$ Wilm, M.S., Mann, M. Electrospray and Taylor-Cone theory, Dole's beam of macromolecules at last? Int J Mass Spectrom lon Process 136, 167-180 (1994)

42 Wilm, M.S., Mann, M. Analytical properties of the nanoelectrospray ion source. Anal Chem 68, 1-8 (1996)

${ }^{43}$ Harris, D.C. Quantitative chemical analysis $7^{\text {th }}$ Ed. W.H Freeman and Co. 484 (2007)

${ }^{44}$ Dodonov, A.F., Chernushevich, I.V., Dodonova, T.F., Raznikov, V.V, Tal'roze, V.L. USSR Patent 1681340A1, February 1987

${ }^{45}$ Dodonov, A.F., Chernushevich, I.V., Laiko, V.V. Extended Abstracts, $12^{\text {th }}$ International Mass Spectrometry Conference, Amsterdam, 1991, 153

${ }^{46}$ Mirgorodskaya, O.A., Shevchenko, A.A., Chernushevich, I.V., Dodonov, A.F., Miroshnikov, A.I. Anal Chem, 66, 99-107, (1994) 
${ }^{47}$ Chernushevich, I.V., Ens, W., Standing, K.G. Orthogonal-Injection TOFMS for analyzing biomolecules. Anal Chem, 4, 452-461, (1999)

${ }^{48}$ Krutchinsky, A.N., Chernushevich, I.V., Spicer, V., Ens, W., Standin, K.G. J Am Soc Mass Spectrom. 9, 569-579, (1998)

${ }^{49}$ Wiza, J.L. Nucl Instrum Methods, 162, 587-601, (1979)

${ }^{50}$ Stewart, I.I. Spectrochim Acta B, 1999, 54, 1649-1695

${ }^{51}$ Schneider, B.B., Chen, D.D. Anal Chem, 72, 791-799 (2000)

${ }^{52}$ Chernushevich, I.V., Loboda, A.V., Thomson, B.A. J Mass Spectrom, 36, 849-865 (2001)

53 de Hoffman, E. Tandem Mass Spectrometry: a Primer. J Mass Spectrom 31, 129-137 (1996)

${ }^{54}$ Hunt, D.F., Yates, J.R.R., Shabanowitz, K., Winston, S., Hauer, C.R. Protein sequencing by tandem mass spectrometry. Proc Natl Acad Sci USA 83, 6233-6237 (1986)

${ }^{55}$ Brugger, B., Erben, G., Sandhoff, R., Wieland, F.T., Lehmann, W.D. Quantitative analysis of biological membrane lipids at the low picomole level by nano-electrospray ionization tandem mass spectrometry. Proc Natl Acad Sci USA 94, 2339-2344, (1997)

${ }^{56}$ Retra, K., Bleijerveld, OB., van Gestel R.A., Tielens, A.G., van Hellmond, J.J., Brouwers, J.F. A simple and universal method for the separation and identification of phospholipid molecular species. Rapid Commun Mass Spectrom 22, 1853-1862, (2008)

${ }^{57}$ Hsu, F.F., Turk, J. Structural determination of sphingomyelin by tandem mass spectrometry with electrospray ionization. J Am Soc Mass Spectrom. 11, 437-449, (2000)

${ }^{58} \mathrm{Hsu}$, F.F., Turk, J. Electrospray ionization/tandem quadrupole mass spectrometric studies on phosphatidylcholines: the fragmentation processes. J Am Soc Mass Spectrom 14, 352-363, (2003)

${ }^{59}$ Proctor, L.D., Warr, A.J. Org Proc Res Dev. 102, 7303-7308 (1980)

60 Little, J.L. Derivatisation of carboxylic acids with diazomethane and trimethylsilyldiazomethane: convenient methods and artefacts; http://userpage.chemie.fuberlin.de/ tlehmann/krebs/files_diazoalkanes.pdf [accessed 8 December 8, 2013]

${ }^{61}$ Aldai, N., Murray, B.E., Najera, A.I., Troy, D.J., Osoro, K.J. Sci Food Agric 85, 1073-1083 (2005)

${ }^{62}$ Wasslen, K.V. Novel Microfluidic Methods to Methylate Peptides and Permit Quantitative Analysis using Tandem Mass Spectrometry. Undergraduate thesis. Carleton University, Ottawa, Ontario, 2011

${ }^{63}$ Schlenk, H., Gellerman, J.L. Anal Chem 32, 1412-1414 (1960)

${ }^{64}$ Graff, G., Anderson, L.A., Jaques, L.W., Scannell, R.T. Chemistry and Physics of lipids, 53, 2736, (1990)

65 Mueller, H.W. Journal of Chromatography B, 679, 208-209 (1996)

${ }^{66}$ Lin, C., Blank, E.W., Ceriani, R.L., Baker, N. Evidence of extensive phospholipid fatty acid methylation during the assumed selective methylation of plasma free fatty acids by diazomethane. Lipids 26, 548-552 (1991)

${ }^{67}$ Smith, G.A., Montecucco, C., Bennett, J.P. Isotopic Labeling of phosphatidylcholine in the choline moiety. Lipids 13, 92-94 (1978)

68 Liebich, H. M.; Forst, C. J Chromatogr, 338, 33-40 (1985)

${ }^{69}$ Kuhn, R. R., H. W. Chemische Berichte, 85, 38-45 (1952)

70 Laremore, T.N., Weber, D.M., Choma, C.T. Rapid Commun Mass Spectrom 19, 2045-2054 (2005) 
${ }^{71}$ Simmons, B.L., Wang, G., Shen, R-F., Knepper, M.A. Rapid Commun Mass Spectrom 20, 24632477 (2006)

72 Blagojevic, V.; Zhidkov, N.; Tharmaratnam, S.; Pham, V. T.; Kaplan, H.; Bohme, D. K. Analyst, 135, 1456-1460 (2010)

73 Park, Y., Albright, K.J., Cai, Z.Y., Pariza, M.W., J Agric Food Chem 43, 1158-1164 (2001)

${ }^{74}$ Lee, J.W., Nishiumi, S., Yoshida, M., Fukusaki, E., Bamba, T. J Chromatogr A 1279, 98-107 (2013)

75 T. de Boer, J., Backer, H. J. Organic Syntheses, 34, 96. (1954)

${ }^{76}$ Sigma-Aldrich, in Aldrich Technical Bulletin AL-180 [Online]. Sigma-Aldrich, St. Louis, 2007

77 Ethier, M., Hou, W., Duewel, H. S., Figeys, D. J Proteome Res 5, 2754-2759 (2006)

${ }^{78}$ Hou, W., Ethier, M., Smith, J. C., Sheng, Y., Figeys, D. Anal Chem 79, 39-44 (2007)

${ }^{79}$ Liuni, P., Rob, T., Wilson, D. J. Rapid Commun Mass Spectrom, 24, 315-320 (2010)

${ }^{80}$ Zhou, H., Hou, W., Denis, N. J., Zhou, H., Vasilescu, J., Zou, H., Figeys, D. J Proteome Res, 8, 556-566 (2009)

${ }^{81}$ Zhou, H.,Elisma, F., Denis, N. J., Wright, T. G., Tian, R., Zhou, H., Hou, W., Zou, H., Figeys, D. J Proteome Res, 9, 1279-1288 (2010)

${ }^{82}$ Wang, F., Chen, R., Zhu, J., Sun, D., Song, C., Wu, Y., Ye, M., Wang, L., Zou, H. Anal Chem, 82, 3007-3015 (2010)

${ }^{83}$ Leib, R. D., Flick, T. G., Williams, E. R. Anal Chem, 81, 3965-72 (2009)

${ }^{84}$ Boersema, P. J., Raijmakers, R., Lemeer, S., Mohammed, S., Heck, A. J. Nat Protoc, 4, 484-494 (2009)

85 Miyagi, M., Rao, K. C. Mass Spectrom Rev, 26, 121-136 (2007)

${ }^{86}$ Ong, S. E., Blagoev, B., Kratchmarova, I., Kristensen, D. B., Steen, H., Pandey, A., Mann, M. Mol Cell Proteomics, 1, 376-386 (2002)

${ }^{87}$ Kovanich, D., Cappadona, S., Raijmakers, R., Mohammed, S., Scholten, A., Heck, A. J. Anal Bioanal Chem, 404, 991-1009 (2012)

${ }^{88}$ Gygi, S. P., Rist, B., Gerber, S. A., Turecek, F., Gelb, M. H., Aebersold, R. Nat Biotechnol, 17, 994-999 (1999)

${ }^{89}$ Ross, P. L., Huang, Y. N., Marchese, J. N., Williamson, B., Parker, K., Hattan, S., Khainovski, N., Pillai, S., Dey, S., Daniels, S., Purkayastha, S., Juhasz, P., Martin, S., Bartlet-Jones, M., He, F., Jacobson, A., Pappin, D. J. Mol Cell Proteomics, 3, 1154-1169 (2004)

90 Thompson, A., Schafer, J., Kuhn, K., Kienle, S., Schwarz, J., Schmidt, G., Neumann, T., Johnstone, R., Mohammed, A. K., Hamon, C. Anal Chem, 75, 1895-1904 (2003)

${ }^{91}$ Wang, M., You, J., Bemis, K. G., Tegeler, T. J., Brown, D. P. Brief Funct Genomic Proteomic, 7, 329-339 (2008)

92 Higgs, R. E., Knierman, M. D., Gelfanova, V., Butler, J. P., Hale, J. E. J Proteome Res, 4, 14421450 (2005)

${ }^{93}$ Strain, H. H. Analytical Chemistry, 30, 620-629 (1958)

94 Turtoi, A., Mazzucchelli, G. D., De Pauw, E. Talanta, 80, 1487-1495 (2010)

${ }^{95}$ Russell, J. D., Hilger, R. T., Ladror, D. T., Tervo, M. A., Scalf, M., Shortreed, M. R., Coon, J. J., Smith, L. M. Anal Chem, 83, 2187-2193 (2011)

${ }^{96}$ Vollmer, S., Astorga-Wells, J., Alvelius, G., Bergman, T., Jornvall, H. Anal Biochem, 374, 154162 (2008) 
${ }^{97}$ Filiou, M. D., Martins-de-Souza, D., Guest, P. C., Bahn, S., Turck, C. W. Proteomics, 12, 736747 (2012)

${ }^{98}$ Flaherty, S., Wark, S., Street, G., Farley, J. W., Brumley, W. C. Electrophoresis, 23, 2327-2332 (2002)

${ }^{99}$ Sammakia, T., in e-EROS Encyclopedia of Reagents for Organic Synthesis [Online]. Wiley, 2001

${ }^{100}$ Smith, J. C., Northey, J. G., Garg, J., Pearlman, R. E., Siu, K. W. Journal of proteome research 2005, 4. 909-19, DOI: 10.1021/pr050013h.

${ }^{101}$ Bras, N. F., Perez, M. A. S., Fernandes, P. A., Silva, P. J., Ramos, M. J. Journal of Chemical Theory and Computation 7, 3898-3908 (2011)

102 Mujika, J. I., Ugalde, J. M., Lopez, X. Theoretical Chemistry Accounts 128, 477-484 (2011)

${ }^{103}$ Clayden, J., Greeves, N., Warren, S., Wothers, P. Organic Chemistry. Oxford University Press. (2001)

${ }^{104}$ Dawson, R.M.C. et al., Data for Biochemical Research, Oxford, Clarendon Press, (1959)

${ }^{105}$ Kumler, W.D., Eiler, J.J. J Am Chem Soc 65, 2355 (1943)

${ }^{106}$ Shimizu T. Lipid mediators in health and disease: Enzymes and receptors as therapeutic targets for the regulation of immunity and inflammation. Annu Rev Pharmacol Toxicol 3, 3 (2008)

${ }^{107}$ Bosetti F. Arachidonic acid metabolism in brain physiology and pathology: Lessons from genetically altered mouse models. J Neurochem 102, 577-586 (2007)

${ }^{108}$ Meyer zu Heringdorf D, Jakobs KH. Lysophospholipid receptors: Signalling, pharmacology and regulation by lysophospholipid metabolism. Biochim Biophys Acta 1768, 923-940 (2007) ${ }^{109}$ Kirschnek S, Paris F, Weller M, Grassme H, Ferlinz K, Riehle A, Fuks Z, Kolesnick R, Gulbins E. CD95-mediated apoptosis in vivo involves acid sphingomyelinase. J Biol Chem 275, 2731627323 (2000)

110 Maestre, I., Jordan, J., Calvo, S., Reig, J.A., Cena, V., Soria, B., Prentki, M., Roche, E.

Mitochondrial dysfunction is involved in apoptosis induced by serum withdrawal and fatty acids in the $\beta$-cell line Ins-1. Endocrinology 144, 335-345 (2003)

${ }^{111}$ Lemmon MA. Membrane recognition by phospholipid-binding domains. Nat Rev Mol Cell Biol 9, 99-111 (2008)

${ }^{112}$ Vicinanza M, D'Angelo G, Di Campli A, De Matteis MA. Function and dysfunction of the PI system in membrane trafficking. EMBO J 27, 2457-2470 (2008)

113 Kita Y, Ohto T, Uozumi N, Shimizu T. Biochemical properties and pathophysiological roles of cytosolic phospholipase A2s. Biochim Biophys Acta 1761, 1317-1322 (2006)

${ }^{114}$ Sanchez-Mejia RO, Newman JW, Toh S, Yu GQ, Zhou Y, Halabisky B, Cisse M, Scearce-Levie K, Cheng IH, Gan L, Palop JJ, Bonventre JV, Mucke L. Phospholipase A2 reduction ameliorates cognitive deficits in a mouse model of Alzheimer's disease. Nat Neurosci 11, 1311-1318 (2008)

${ }^{115}$ Han X, Abendschein DR, Kelley JG, Gross RW. Diabetes-induced changes in specific lipid molecular species in rat myocardium. Biochem J 352, 79-89 (2000)

${ }^{116}$ Distler U, Hulsewig M, Souady J, Dreisewerd K, Haier J, Senninger N, Friedrich AW, Karch H, Hillenkamp F, Berkenkamp S, Peter-Katalinic J, Muthing J. Matching IR-MALDI-o-TOF masS spectrometry with the TLC overlay binding assay and its clinical application for tracing tumorassociated glycosphingolipids in hepatocellular and pancreatic cancer. Anal Chem 80, 18351846 (2008) 
${ }^{117}$ Haroldsen PE, Clay KL, Murphy RC. Quantitation of lyso-platelet activating factor molecular species from human neutrophils by mass spectrometry. J Lipid Res 28, 42-49 (1987)

${ }^{118}$ Ekroos K, Chernushevich IV, Simons K, Shevchenko A. Quantitative profiling of phospholipids by multiple precursor ion scanning on a hybrid quadrupole time-of-flight mass spectrometer.

Anal Chem 74, 941-949 (2002)

${ }^{119}$ Wenk MR, Lucast L, Di Paolo G, Romanelli AJ, Suchy SF, Nussbaum RL, Cline GW, Shulman GI, McMurray W, De Camilli P. Phosphoinositide profiling in complex lipid mixtures using electrospray ionization mass spectrometry. Nat Biotechnol 21, 813-817 (2003)

${ }^{120}$ Liebisch G, Drobnik W, Lieser B, Schmitz G. High-throughput quantification of lysophosphatidylcholine by electrospray ionization tandem mass spectrometry. Clin Chem $\mathbf{4 8}$, 2217-2224 (2002)

${ }^{121}$ Whitehead SN, Hou W, Ethier M, Smith JC, Bourgeois A, Denis R, Bennett SA, Figeys D. Identification and quantitation of changes in the platelet activating factor family of glycerophospholipids over the course of neuronal differentiation by high-performance liquid chromatography electrospray ionization tandem mass spectrometry. Anal Chem 79, 8539-8548 (2007)

${ }^{122}$ Goto-Inoue, N., Hayasaka, T., Taki, T., Gonzalez, T.V., Setou, M. J Chromatogr A 1216, 70967101 (2009)

${ }^{123}$ Shields, S. W. J., Manthorpe, J. M. Efficient, Scalable, and Economical Preparation of tris(Deuterium)- and ${ }^{13} \mathrm{C}$-Labelled $N$-Methyl- $N$-Nitroso- $p$-Toluenesulfonamide (Diazald ${ }^{\circledR}$ ) and their Conversion to Labelled Diazomethane. Submitted. 